Heat Transfer Across Dielectric-metallic INTERFACES AND THIN LAYERS AT LOW AND ULTRA-LOW TEMPERATURES

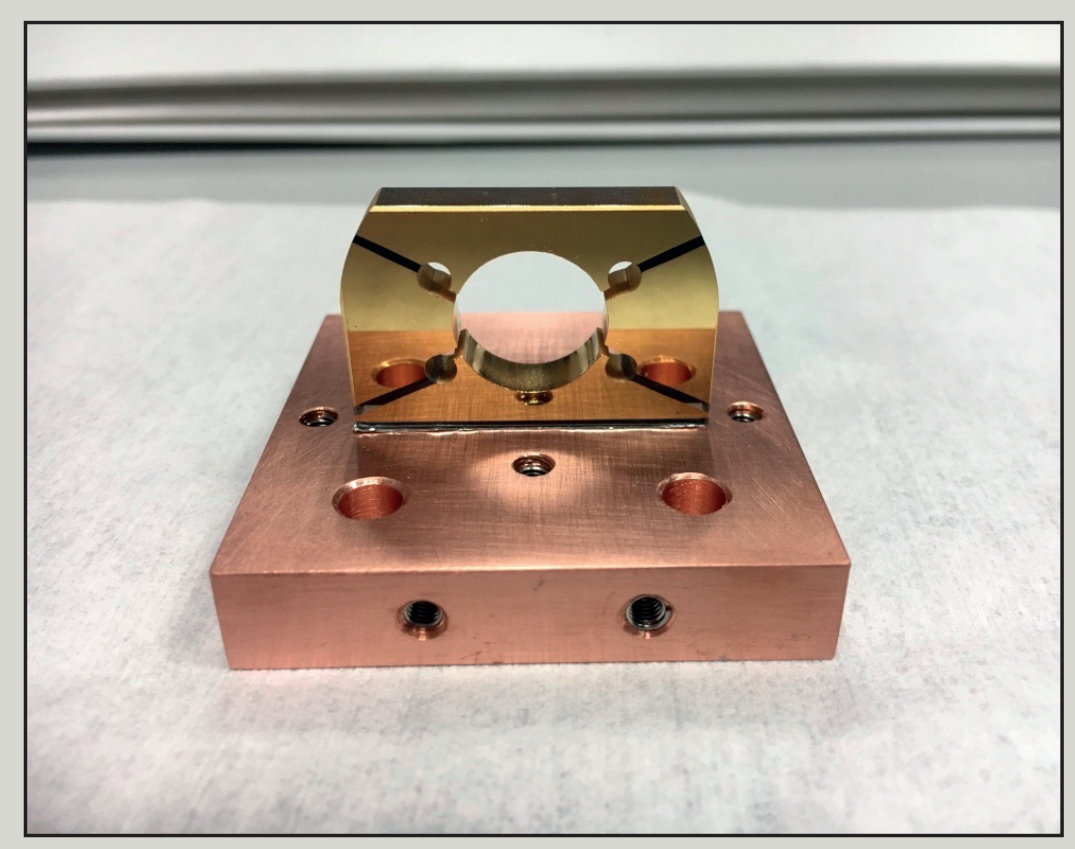

JOANNA LIBERADZKA 


\title{
Heat Transfer Across DiELECTRIC-METALLiC INTERFACES AND THIN LAYERS AT LOW AND Ultra-LOW TEMPERATURES
}

\author{
Joanna Liberadzka
}


Graduation committee:

Chairman Prof. dr. J.L. Herek

Supervisor Prof. dr. ir. H.J.M. ter Brake

Co-supervisor Dr. rer. nat. T. Köttig

Members $\quad$ Prof. dr. ing. B. van Eijk

Prof. dr. ir. H.H.J. ten Kate

Prof. dr. rer. nat. R. Nawrodt

Prof. dr. ir. T.H. Oosterkamp

Special expert Dr. G. Vermeulen

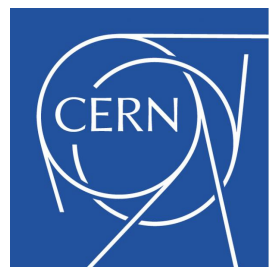

The research described in this thesis was founded by and carried out at CERN, The European Organisation for Nuclear Research, Technology Department (TE), Cryogenics Group (CRG), Cryolab \& Instrumentation Section (CI).

Heat Transfer Across Dielectric-metallic Interfaces and Thin Layers at Low and Ultra-low Temperatures

J. Liberadzka

$\mathrm{PhD}$ thesis, University of Twente, The Netherlands

ISBN 978-90-365-4704-8

DOI 10.3990/1.9789036547048

URL https://doi.org/10.3990/1.9789036547048

Printed by Ipskamp Printing, Enschede, The Netherlands

Cover by J. Liberadzka

(C) J. Liberadzka, Enschede, 2019 


\section{Heat Transfer Across DiELECTRIC-METALLIC INTERFACES AND THIN LAYERS AT LOW AND Ultra-LOW TEMPERATURES}

\section{DISSERTATION}

to obtain

the degree of doctor at the University of Twente, on the authority of the rector magnificus, prof. dr. T.T.M. Palstra, on account of the decision of the graduation committee, to be publicly defended on Thursday, the $24^{\text {th }}$ of January 2019 at 14:45

by

\section{Joanna Liberadzka}

born on the $30^{\text {th }}$ of January 1988

in Sochaczew, Poland 
This thesis has been approved by:

Prof. dr. ir. H.J.M. ter Brake (PhD supervisor)

Dr. rer. nat. T. Köttig (co-supervisor) 


\section{Contents}

\begin{tabular}{lll}
\hline & Introduction & $\mathbf{7}$
\end{tabular}

1.1 The AEgIS experiment at CERN . . . . . . . . . . . . . . . . . 7

1.2 Electrode requirements . . . . . . . . . . . . . . . 8

1.3 Electrode development . . . . . . . . . . . . . . . . . . . . . . . . . . . 9

1.4 Research goals . . . . . . . . . . . . . . . . 10

2 Theory of heat transfer at low temperatures 13

2.1 Heat capacity of solids . . . . . . . . . . . . . 13

2.1 .1 Dielectric crystals . . . . . . . . . . . . . . . . . . . . . . . . . . . 13

2.1 .2 Metals . . . . . . . . . . . . . . . . . 15

2.2 Thermal conductivity . . . . . . . . . . . . . 16

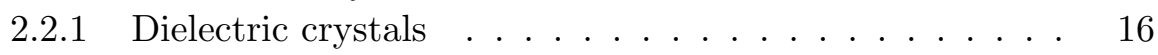

$2.2 .2 \quad$ Thermal conductivity of metals . . . . . . . . . . . . 18

2.3 Superconductivity and heat transfer . . . . . . . . . . . . . . 19

2.4 Thermal boundary resistance . . . . . . . . . . . . . 22

$2.4 .1 \quad$ Acoustic Mismatch Model . . . . . . . . . . . . . . . . . 23

2.4 .2 Electron coupling to surface waves . . . . . . . . . . 25

$2.4 .3 \quad$ Diffuse Mismatch Model . . . . . . . . . . . . . . . . . . 26

2.4 .4 Advanced higher temperature models . . . . . . . . . . 28

2.5 Thermal conductivity of thin layers . . . . . . . . . . . . . . . 30

2.6 Thermal diffusivity . . . . . . . . . . . . . . . 35

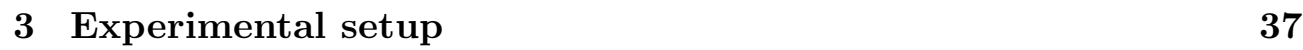

3.1 Mock-up of the electrode . . . . . . . . . . . . . . . . . . 37

3.1 .1 Copper - indium - sapphire sandwich . . . . . . . . . . . 37

$3.1 .2 \quad$ Sandwich with $\mathrm{Ti}$ - Au coating on the sapphire . . . . . 39

3.1 .3 Verification of microstructure and elemental distribution in sandwich thin films . . . . . . . . . . . . . . . . . . . 40

3.2 Ultra-low temperature measurements in the Dilution Refrigerator 44

3.2.1 The principle of achieving ultra-low temperatures. . . . 44

3.2.2 CERN Cryolab Dilution Refrigerator . . . . . . . . . . . 45

3.3 Mechanical crycoolers . . . . . . . . . . . . . 47 
3.3.1 Stirling and Gifford-MacMahon cycles . . . . . . . . . . 49

3.3.2 $\quad$ Pulse Tube Refrigerator (PTR) _ . . . . . . . . . . 50

$3.3 .3 \quad$ Cryocoolers used in the CERN Cryolab . . . . . . . . . 51

\begin{tabular}{|lll}
4 & Measurement methodology, instrumentation and accuracy & 53
\end{tabular}

4.1 Steady state measurement method . . . . . . . . . . . 53

4.1.1 Sensors used for the ultra-low temperature measurements 56

$4.1 .2 \quad$ Superconducting Fixed Point Device SRD1000 . . . . 57

$4.2 \quad$ Sensor calibration in the ultra-low temperature range. . . . . . 60

4.2 .1 Thermal cycling of temperature sensors . . . . . . . 60

$4.2 .2 \quad$ Bare chips . . . . . . . . . . . . . . . . 60

4.2 .3 AA packages . . . . . . . . . . . . . . . 63

4.3 Estimation of the steady state measurement uncertainty at ultra-

low temperatures . . . . . . . . . . . . . . . 64

4.3 .1 Temperature measurement uncertainty . . . . . . . . . 64

$4.3 .2 \quad$ Power measurement uncertainty $\ldots \ldots \ldots$. . . . . . 66

$4.3 .3 \quad$ Uncertainty of the interface thermal resistivity . . . . . 66

4.4 Transient measurement method . . . . . . . . . . . . 68

4.4 .1 Overview of the measurement methods. . . . . . . . . 68

$4.4 .2 \quad$ Measurement chain analysis . . . . . . . . . . . . . . 69

4.4.3 Accuracy of the heater - temperature sensor phase shift

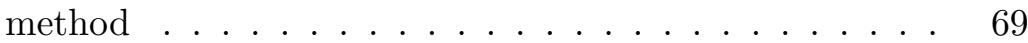

4.4.4 Accuracy of the temperature sensor - temperature sensor phase shift method . . . . . . . . . . . . 70

$4.4 .5 \quad$ Validation of the amplitude attenuation method and the threshold frequency. . . . . . . . . . . . . . 71

4.5 Estimation of the uncertainty in the low temperature thermal conductivity measurement . . . . . . . . . . . 72

5 Mathematical model $\quad \mathbf{7 5}$

5.1 Copper - indium sc - copper connection . . . . . . . . . 78

5.2 Copper - indium nc-sapphire connection . . . . . . . . . . 81

5.3 Copper - indium sc - sapphire connection . . . . . . . . . 84

5.4 Copper - indium nc - gold - titanium nc - sapphire . . . . . . . 88

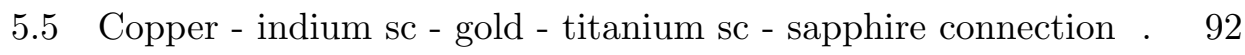

$\begin{array}{lll}6 & \text { Measurement results } & 97\end{array}$

6.1 Steady state measurements of the copper - indium - sapphire

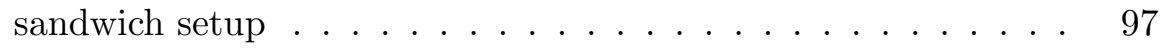

$6.1 .1 \quad$ Thermal resistivity of the mechanically compressed setup 98

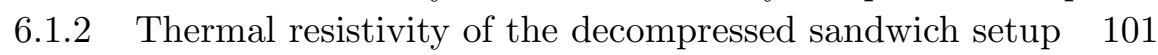

$6.1 .3 \quad$ Sandwich without the clamping structure . . . . . . . . 104

6.1 .4 Comparison with the model . . . . . . . . . . . . . 108 
6.2 Steady state measurements of the copper - indium - gold - titanium - sapphire sandwich setup . . . . . . . . . . . . . 112

6.2 .1 Measurements with an external magnetic field. . . . . . 113

6.2.2 Measurements without an external magnetic field . . . . 115

$6.2 .3 \quad$ Comparison with the model . . . . . . . . . . . . 118

6.3 Transient measurements in the low temperature range from $3 \mathrm{~K}$ to $30 \mathrm{~K} \ldots \ldots \ldots \ldots . \ldots \ldots 121$

6.3 .1 Thermal diffusivity of the copper - indium - sapphire sandwich setup . . . . . . . . . . . 122

6.3 .2 Thermal diffusivity of the copper - indium - gold - titanium - sapphire sandwich setup . . . . . . . . . . 124

6.3 .3 Theoretical estimation of the low temperature thermal conductivity and thermal diffusivity . . . . . . . . . 126

6.4 Transient measurements at ultra-low temperatures . . . . . . . 131

6.4.1 Thermal diffusivity of the copper - indium - gold - titanium - sapphire sandwich setup . . . . . . . . . . 131

6.4 .2 Theoretical estimation of the ultra-low temperature thermal diffusivity ............... 133

6.5 Conclusions drawn from the measurement results . . . . . . . . 135

$\begin{array}{lll}7 & \text { Ultra-cold electrode design and thermal performance } & 137\end{array}$

7.1 Stress analysis and the quality of the indium bond in the old electrode. ................... . . 137

7.2 Thin layers and their influence on the thermal performance of the electrode ................... . 140

7.3 New design of the electrode and its manufacturing . . . . . . . 141

7.4 Ultra-low temperature measurements . . . . . . . . . . . . . . 145

$7.4 .1 \quad$ Steady state measurement results . . . . . . . . . . . . . 146

7.4.2 Response to transient heat loads . . . . . . . . . . . . . 148

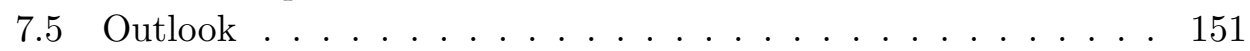

\begin{tabular}{lll}
\hline Conclusions & 153 \\
\hline
\end{tabular}

\begin{tabular}{ll}
\hline Appendix & 157
\end{tabular}

\begin{tabular}{lr}
\hline Bibliography & 159
\end{tabular}

\begin{tabular}{ll}
\hline Summary & 167
\end{tabular}

\begin{tabular}{ll}
\hline Samenvatting & 171
\end{tabular}

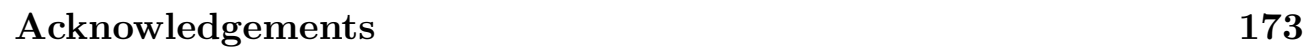




\section{Chapter 1}

\section{Introduction}

The European Organization for Nuclear Research (CERN) supports research in the field of fundamental particle science. Besides the experiments located at the ring of the biggest accelerator - the Large Hadron Collider, there are many other experiments investigating physics from cosmic rays to supersymmetry. The properties of antimatter are studied in a dedicated facility, which is the Antimatter Factory, located in the Antiproton Decelerator (AD) complex.

\subsection{The AEgIS experiment at CERN}

The Antimatter Experiment: gravity, Interferometry, Spectroscopy (AEgIS) [1] is one of a few experiments studying the properties of antimatter at CERN. The goal of the experiment is a first direct measurement of the antihydrogen's acceleration in free fall in the Earth's gravitational field within $1 \%$ precision. The universality of free fall has been well measured for normal matter [2], but a matter - antimatter configuration has never been studied before, and different physical models predict a different result on the experiment. The AEgIS is based at CERN's antimatter factory, where it uses a beam of antiprotons from the antiproton decelerator ring and combines it with positronium to create antihydrogen.

Positronium consists of pairs of electrons and positrons, pairwise whirling around each other. Positrons are provided to the experimental area by a $\beta^{+}$ decay of a ${ }^{22} \mathrm{Na}$ source. Then positronium is obtained by shooting positrons on a nanostructural porous material, where they "catch" electrons. Before the positronium merges with antiprotons, it is excited to a higher Rydberg state with a laser. The antiprotons are trapped in the electromagnetic field of a Penning trap, where the antihydrogen is created by charge exchange, as presented in figure 1.1 [2]. Then the antihydrogen particles are accelerated horizontally, they pass through a series of gratings of a Moire deflectometer, and finally they annihilate on a detector plane. Knowing the place where they annihilated and 

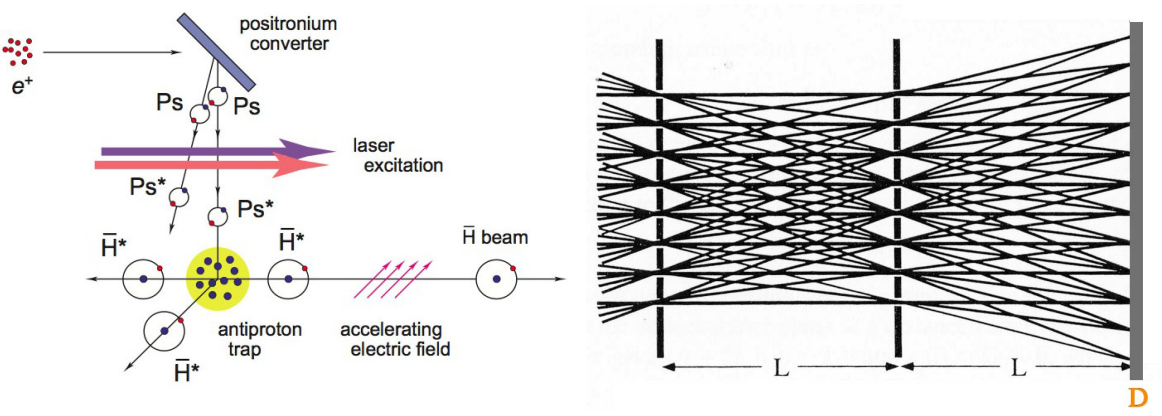

Figure 1.1: Principle of the AEgIS experiment and the Moire deflectometer 3 .

measuring their time of flight, one can calculate whether the gravitational acceleration is the same as for normal matter. As a very light atom, antihydrogen drops only several micrometers, so it is necessary to cool the particles down to significantly reduce their thermal movement and thereby increase the precision of the measurement [2]. Therefore a package of high voltage electrodes is supposed to be placed on a mixing chamber (MC) of a dilution refrigerator (DR), providing continuous cooling power and keeping the electrodes below $100 \mathrm{mK}$.

The CERN Central Cryogenic Laboratory, called Cryolab, was asked to design a set of electrodes fulfilling a series of very demanding constraints, out of which the most difficult one is the need of thermally anchoring the electrodes to the mixing chamber of a dilution refrigerator and at the same time keeping them electrically insulated.

\subsection{Electrode requirements}

In the ultra-cold region of AEgIS, there are to be 10 electrically insulated electrodes, some of them divided into 4 independent sectors. The requirements can be summarised as follows:

- The electrodes should be cooled to temperatures below $100 \mathrm{mK}$ in the second half of the $100 \mathrm{~s}$ AD cycle, despite the introduced heat load coming along wires, from radiation and annihilation of antimatter. The estimation predicts a pulse of $10^{-5} \mathrm{~J}$ lasting $1 \mu \mathrm{s}$, entering the region at the beginning of each $100 \mathrm{~s}$ cycle. That would give an enormous (at $100 \mathrm{mK}$ ) heat load of $10 \mathrm{~W}$. We assume that the ideal pulse will in reality spread over time resulting in $10 \mu \mathrm{W}$ during a time period of 1 second. The connection to the mixing chamber should guarantee a suitable thermal anchoring statically and dynamically.

- Some of the electrodes will be divided into 4 electrically insulated sectors. The insulation between sectors and neighbouring electrodes must 
withstand a potential difference of $1 \mathrm{kV}$. The division into sectors must be done in such a way, that the trapped particles have no direct line of sight to the dielectric, but only to polished, metallic surfaces.

- The electrodes should be manufactured with great precision, made of radiation hard materials, and be compliant with ultra-high vacuum of $10^{-12}$ mbar. Any outgassing from the electrode itself or its connection to the mixing chamber would cause a degradation of vacuum and annihilation of antimatter. The direct interface surface to the cold source is considered to be covered by the design.

- Finally yet importantly, the design should take into account that there is a limited space in the ultra-cold region. The electrode and the mixing chamber, including required thermal shields at $1.5 \mathrm{~K}$ and $300 \mathrm{mK}$, have to fit in the magnet cold bore of $90 \mathrm{~mm}$ diameter.

The fact that the electrodes should be highly thermally conducting and at the same time well electrically insulated makes the task very demanding. Except for dealing with a set of contradictive requirements, we are working in the environment of ultra-high vacuum and ultra-low temperatures. A few iterations in the development process of the electrodes were necessary, to reach the final electrode design and the thermalisation strategy.

\subsection{Electrode development}

In the previous research in the Cryolab the conceptual design of the AEgIS electrodes was studied, and a series of measurements to test their performance was conducted. In the initial design, the electrode consisted of four separate copper elements with dielectric spacers separating them. Thomas Eisel [4 manufactured one electrode according to this design, using sapphire as the spacer material (figure 1.2 on the left). Unfortunately, a construction of that type turned out to be almost impossible to assemble with the required precision.

After the first attempt, the Cryolab team realised that in fact an electrode does not have to be made of a bulk piece of copper. Sapphire, as a perfect crystal, is a relatively good thermal conductor at low temperatures (see fig. 2.4), and as a dielectric it provides the required electrical insulation. The idea arose to manufacture the whole geometry from one precisely machined sapphire crystal, and then create the electrode sectors by sputtering gold on the sapphire base. That approach guarantees much higher manufacturing and assembling precision. G. Burghart designed a small and neat electrode, made of sapphire covered with gold (figure 1.2 on the right), which could fit in the limited space of the AEgIS cold bore. For the future application, the electrode would be attached to the mixing chamber with an intermediate layer of indium foil. Indium has a particular property of "cold welding" easily, when two 

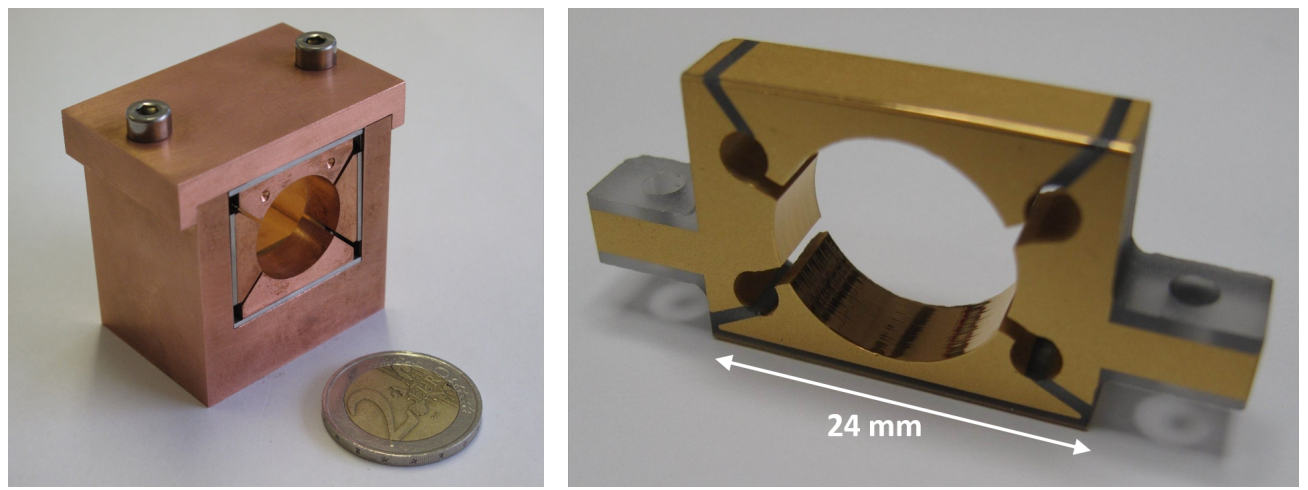

Figure 1.2: Electrodes made by Thomas Eisel (left) and Gerhard Burghart (right) 4 .

nonoxidized pure indium surfaces are compressed [5, 6]. Under compression it shows also quite a good adherence to nonoxidized surfaces of other metals. The thermal performance of this electrode, attached to the copper lid of a mixing chamber with a layer of indium in between, was measured in the CERN Cryolab Dilution Refrigerator, and unfortunately the performance was found to be 4 times smaller than the estimation based on the preliminary results [4,7], what motivated further research.

\subsection{Research goals}

This work investigates the limitations of heat transport in a complex shape dielectric crystal and in a dielectric - metal - superconductor sandwich structure. Especially, it focuses on the problem of the thermal boundary resistance at a dielectric-metal interface in the milliKelvin range, which we expect to be the main thermal bottleneck for the application.

Thus we can formulate the following research goals:

- Measurement of a sapphire - indium - copper sandwich thermal resistivity with investigation of influencing factors, such as:

- compression force applied to the sandwich and mechanical stress in materials, especially in the dielectric,

- magnetic field and the switch between normal and superconducting state,

- thermal cycling, ageing and the effects of a possible degradation of the thermal properties;

- Measurement of a sapphire - titanium - gold - indium - copper sandwich thermal resistivity with indium and titanium both in the normal and superconducting states; 
- Analysis of the thin layers thermal conductivity and its influence on the whole sandwich structure;

- Qualitative and quantitative description of the phenomena and a formulation of a representative mathematical model;

- Design and test of the ultra-cold electrode for the AEgIS experiment, fulfilling the very strict requirements, described in section 1.2 .

The next chapter summarises all the theory described in literature, necessary for the understanding of the low temperature phenomena observed. A detailed description of the sandwich setup and the apparatus, used for the ultra-low and low temperature measurements, can be found in chapter 3 . An estimation of the measurement uncertainty corresponding to all types of sensors and particular instrumentation used, is described in chapter 4 . The mathematical model created for various configurations of the sandwich setup, along with the theoretical background used for its formulation, is explained in chapter 5. The ultra-low and low temperature measurement results are presented in chapter 6. The new design, manufacturing and evaluation of the thermal performance of the AEgIS electrode is described in chapter 7. In the last chapter 8, all the accomplished tasks are summarised, and the final conclusions are drawn. 


\section{Chapter 2}

\section{Theory of heat transfer at low temperatures}

A profound study of the structure of matter and the heat transfer mechanisms is essential for the understanding of the low temperature heat transfer phenomena at interfaces. Different mechanisms govern the heat transfer in metals and dielectrics, and phenomena exist in the milli-Kelvin temperature range, which are not yet fully understood. What happens in a thin layer of a dielectric, when at ultra low temperature, the corresponding phonon wavelength exceeds the dimension of the sample? For certain configurations of materials, involving thin layers and superconductors, the experimentally verified theoretical description reaches not lower than several hundred milli-Kelvin 8 28. This chapter resumes the established theory, which is necessary to build a model describing the ultra-low temperature behaviour of the studied configurations of the setup.

\subsection{Heat capacity of solids}

\subsubsection{Dielectric crystals}

Various attempts to describe the nature of heat capacity of dielectric solids were made. The most significant are the models presented by Albert Einstein and Peter Debye [29, 30].

Einstein described the vibration of the lattice structure as a series of independent harmonic oscillators with the same frequency. The theory is consistent with the Dulong-Petit law stating that at room temperature the molar specific heat of a given material is experimentally related to the gas constant $C=3 R$, but for low temperature it predicts that the heat capacity decreases faster than experimentally observed 30 .

A model proposed by Peter Debye assumes that the quanta of energy associated with oscillations, called phonons by analogy to photons, propagate in 
the material interacting with each other like gas particles in a box. It assumes also a linear dispersion relation between the wave vector and the frequency of oscillations. Debye introduced the frequency dependent density of states [31]

$$
D(\omega)=\frac{V \omega^{3}}{2 \pi^{2} v^{3}} d \omega
$$

where $v$ is the speed of sound in the material, that in the presence of longitudinal and transverse sound waves can be presented in the form of a Debye velocity

$$
\frac{3}{v_{\mathrm{D}}^{3}}=\frac{1}{v_{l}^{3}}+\frac{2}{v_{t}^{3}} .
$$

It is necessary to introduce a corresponding cut-off frequency $\omega_{D}$ limiting the total number of modes to $3 \mathrm{~N}$

$$
3 N=\int_{0}^{\omega_{\mathrm{D}}} D(\omega) d \omega
$$

The internal energy of lattice vibrations equals [31]:

$$
U(T)=\int_{0}^{\hbar} \hbar \omega D(\omega) f(\omega, T) d \omega,
$$

where $f(\omega, T)$ is a Bose-Einstein distribution function. Inserting the distribution function, one can define the molar heat capacity as [31]:

$$
C_{V}=9 N k_{\mathrm{B}}\left(\frac{T}{\Theta}\right)^{3} \int_{0}^{\hbar \omega_{\mathrm{D}} / k_{\mathrm{B}} T} \frac{x^{4} e^{x}}{\left(e^{x}-1\right)^{2}} d x
$$

where $\Theta$ is the Debye temperature defined as $k_{\mathrm{B}} \Theta=\hbar \omega_{\mathrm{D}}, k_{\mathrm{B}}$ is the Boltzmann constant and $\hbar$ is the reduced Planck constant. Such a formulation predicts correctly the high temperature limit of the specific heat $C_{V}=3 N k_{B}=3 R$. The low temperature behaviour also corresponds to experimental observations with the heat capacity being proportional to the third power of temperature:

$$
C_{V}=\frac{12 \pi^{4}}{5} R\left(\frac{T}{\Theta}\right)^{3}
$$

In the intermediate temperature range the Debye approximation is not very exact [30].

The proportionality of the heat capacity of dielectrics to the third power of temperature $C \propto T^{3}$ is a very important information for ultra-low temperature modelling of the thermal conductivity and the thermal diffusivity, describing the thermal performance of the Cryolab DR sandwich setups and the AEgIS electrodes. 


\subsubsection{Metals}

In metals the contribution of valence electrons to the specific heat is small and linearly proportional to temperature. The corresponding molar specific heat, following 31] can be expressed as:

$$
c_{V}=\gamma T+\beta T^{3}
$$

where

$$
\gamma=\frac{\pi^{2} R k_{B}}{2 \epsilon_{f}}
$$

is the Sommerfeld coefficient characterizing the electronic part of specific heat and $\beta$ the lattice part described in section 2.1.1. For copper, the Fermi energy $\epsilon_{F}=7 \mathrm{eV}$ and the Sommerfeld coefficient $\gamma=5 \cdot 10^{-4} \mathrm{~J} /\left(\mathrm{mol} \mathrm{K}{ }^{2}\right)$. The contributions of both heat carriers are compared at an example of low temperature specific heat of copper [31] in figure 2.1. As one can see, in copper below $4 \mathrm{~K}$ the contribution from electrons to the heat capacity becomes dominant over the lattice contribution. It shows the necessity of including in the mathematical modelling at ultra-low temperatures the contributions from the electron side in multiple metallic layers of the Cryolab DR sandwich setup.

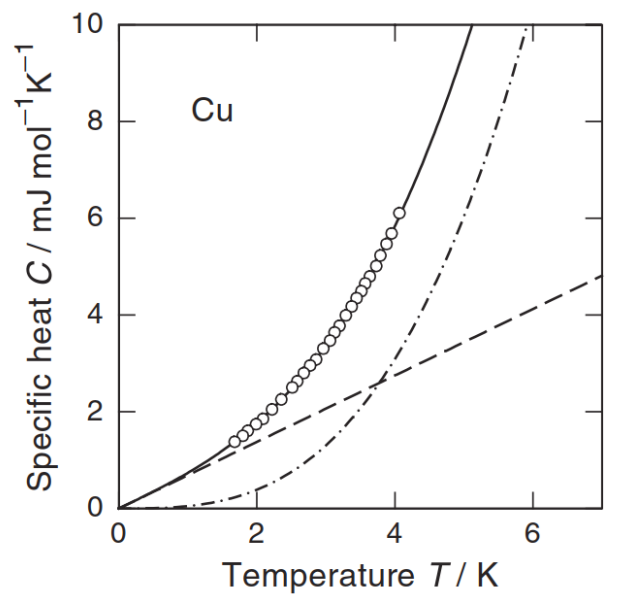

Figure 2.1: Low temperature specific heat of copper from 31. The dashed line represents the electron contribution and the dashed-dotted line the contribution of the lattice. The full line is the sum of electron and phonon contributions. The circles indicate measurement data 19 . Below approx. $4 \mathrm{~K}$ the electron contribution is dominant over the lattice contribution. 


\subsection{Thermal conductivity}

\subsubsection{Dielectric crystals}

The thermal conductivity of dielectric crystals, following the kinetic theory of gases can be expressed by [31]:

$$
\lambda=\frac{1}{3} c_{V} v l,
$$

where $c_{V}$ is the specific heat per unit volume, $v$ mean velocity of the heat carriers and $l$ their mean free path.

The velocity of heat carriers is evaluated by assuming a linear dispersion relation and applying a dominant phonon approximation. Therefore it is equal to the speed of sound in a crystal, and it is rather independent of temperature.

The mean free path is more difficult to evaluate as it depends on the scattering of phonons at imperfections and with other phonons, and is therefore dependent on the density of imperfections and the temperature. While scattering with each other, two phonons can merge into one, or one phonon can decay into two. When the energy of a phonon incident is relatively low, the associated wave vectors remain in the first Brillouin zone [30,31. The sum of energy and quasi-momentum of the phonons is conserved, and the heat flow is not degraded. This type of scattering is called a normal process ( $N$-process). For the normal processes the mean free path is described by the dependence $l_{N} \propto T^{-5}[31]$. For higher energies of the incident phonon, the resulting wave vector is laying outside of the first Brillouin zone, and a reciprocal lattice vector $G$ appears, that shifts it back to the zone. Such a process is called an Umklapp process (U-process), it causes a degradation of the heat flow and a distribution of phonon energies between modes. The mean free path of U-processes is described by the dependence $l_{U} \propto T^{-1} 31$.

There is a whole spectrum of imperfections that can cause the phonons to scatter: point defects, like an intrusion of another atom in a lattice structure, dislocations of the lattice, grain boundaries, and above all a surface "defect" in the form of a finite size of the crystal. Each type of defect influences the mean free path differently in terms of temperature dependence. A detailed description can be found for instance in Low-Temperature Physics by Ch. Enss [31]. As an example the thermal conductivity of sodium fluoride is depicted in figure 2.2. As one can see, at very low temperature, when the mean free path of phonons is long and limited only by the imperfections, the thermal conductivity follows the thermal dependence of specific heat $\lambda \propto c_{V} \propto T^{3}$. When the temperature rises, there are more phonon modes occupied and a higher chance of phonon-phonon scattering. At a certain temperature phononphonon scattering becomes the dominant limiting factor of the heat flow in the material, and the thermal conductivity decreases with increasing temperature (figure 2.2). The overall temperature dependence shows a typical conductivity 
peak around 25 - $50 \mathrm{~K}$ for dielectric crystals. In figure 2.3 one can clearly see the influence of grain boundaries on the thermal conductivity of sapphire, causing a decrease by two orders of magnitude.

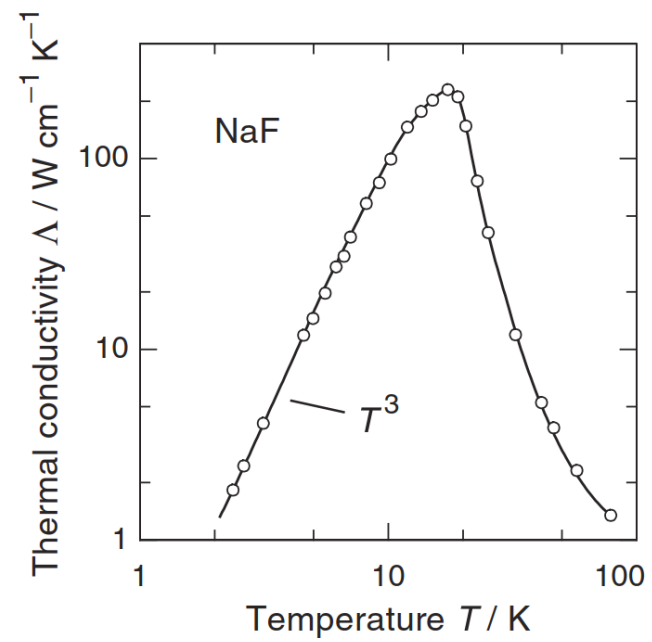

Figure 2.2: Thermal conductivity of NaF from 31 as an example of a rather "perfect" dielectric crystal in log-log scale, showing $T^{3}$ dependence at the low temperature end. At higher temperatures the phonon-phonon scattering dominates the heat transfer and the conductivity decreases with increasing temperature.

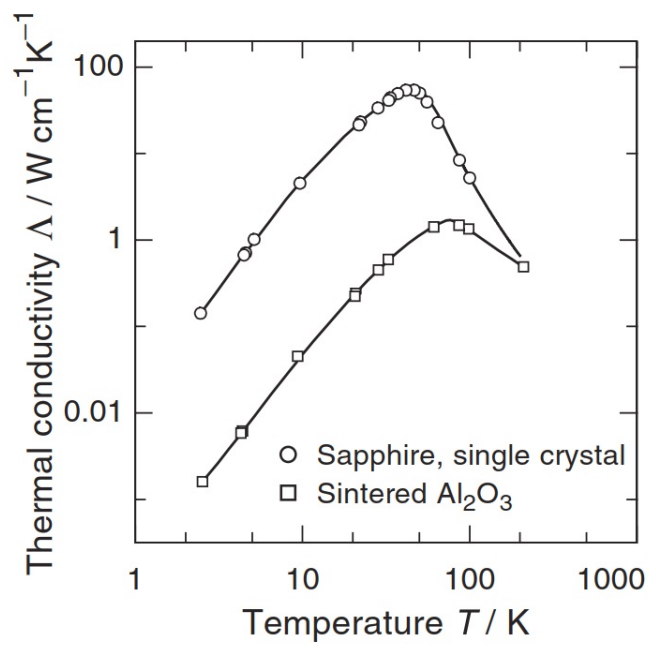

Figure 2.3: Thermal conductivity of sapphire: circles - single crystal; squares - sintered $\mathrm{Al}_{2} \mathrm{O}_{3}$. A two orders of magnitude decrease in thermal conductivity because of a multi-grain structure of the sintered material versus single crystal is clearly visible at temperatures below the conductivity peak. As in the case of $\mathrm{NaF}$ (figure 2.2), above a certain temperature the phononphonon scattering becomes dominant and limits the thermal conductivity. 
The sapphire disks used for measurements in the Cryolab DR are machined from a single crystal and are considered as a perfect crystal structure. At ultralow temperatures, when most of the phonons are frozen out, the only factor that could cause any dislocation and significant phonon scattering is the stress in the material caused by a high mechanical compression force. However, if the force is applied uniformly to the whole disk, one can still talk about the so called ballistic propagation of phonons [32]. The mean free path could even reach the order of $1 \mathrm{~mm}$ corresponding to the thickness of the investigated sapphire disk in the Cryolab DR sandwich setup. At low temperature the thermal conductivity of sapphire is proportional to the specific heat and therefore to the third power of temperature:

$$
\lambda_{\text {sapphire }}^{\text {th }} \propto T^{3} .
$$

\subsubsection{Thermal conductivity of metals}

The electrons play a significant role in the thermal conduction of metals at low temperatures, when there are few phonons left. One can write an expression for the thermal conductivity of electrons by analogy to the lattice:

$$
\lambda_{\mathrm{el}}^{\mathrm{th}}=\frac{1}{3} c_{V}^{\mathrm{el}} v_{\mathrm{F}} l^{\mathrm{el}}
$$

where $c_{V}^{e l}$ is the electronic specific heat and $v_{F}$ is the Fermi velocity, and $l^{e l}$ is the mean free path of electrons. The Fermi velocity is orders of magnitude higher than the speed of sound. For that reason $\lambda_{\text {el }}^{t h}$ can be significant, despite the low value of electronic specific heat. Moreover it has a linear temperature dependence:

$$
\lambda_{\mathrm{el}}^{\mathrm{th}} \propto T
$$

Many researchers state [33 37] that according to the Matthiessen rule [30], the electronic thermal resistivity, which depends on the mobility of electrons as the electrical resistivity, can be presented as a sum of resistivities coming from the imperfections of the lattice, like defects and impurities, plus the "ideal" resistivity caused by electrons scattering on phonons:

$$
\frac{1}{\lambda_{e l}^{t h}}=R_{e l}^{t h}=R_{i m p}^{t h}+R_{p h}^{t h}
$$

The ideal phonon related resistivity should disappear along with the decrease of temperature to near absolute zero:

$$
R_{T->0}^{t h}=R_{i m p}^{t h} .
$$

The Wiedemann-Franz-Lorenz law states that the ratio of thermal to electrical conductivity is proportional to temperature 30 :

$$
\frac{\lambda_{e l}^{t h}}{\sigma}=\frac{\pi^{2}}{3}\left(\frac{k_{B}}{e}\right)^{2} T
$$


At low temperature, when the electric conductivity becomes constant and its value depends only on the imperfections, the thermal conductivity of electrons is proportional to temperature $\lambda_{e l}^{t h} \propto T$, which is the same temperature dependence as derived from the theory, described in equation (2.12).

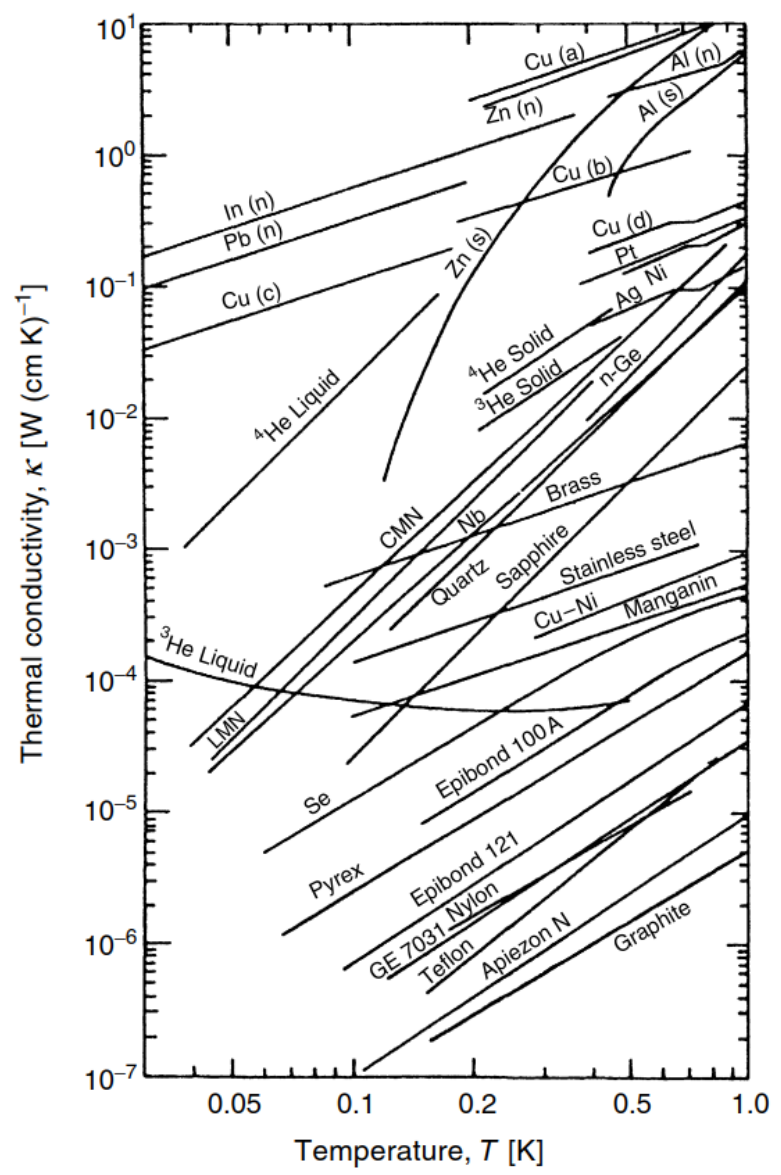

Figure 2.4: Low temperature thermal conductivity of selected materials 38. The conductivity of sapphire is around three orders of magnitude lower than the conductivity of copper, but relatively high compared to other dielectric materials.

The low temperature thermal conductivities of various materials, including copper, indium and sapphire, are presented in figure 2.4. The conductivity of metals is significantly higher than dielectrics, but the conductivity of sapphire is relatively high compared to other dielectric materials.

\subsection{Superconductivity and heat transfer}

The phenomenon of superconductivity, except for being very interesting, is also very important for the thermal properties of materials and structures. First 
discovered by Kammerlingh Onnes in Leiden at a sample of mercury at low temperature [39], it remained not explained theoretically until Ginzburg and Landau presented their theory [40], which explained the macroscopic behaviour of type I superconductors. Later on, Bardeen, Cooper and Schrieffer published their "Microscopic Theory of Superconductivity" [41], which until now remains the best available description of the phenomena. Electrons in superconductors are coupled via lattice interactions in so called "Cooper pairs", creating an energy gap. The pairs behave as bosons, not fermions anymore, and follow Bose-Einstein statistics. They don't interact with the lattice in the same way as in the normal conducting (nc) state, and the electrical resistivity vanishes for DC conditions. Valence electrons in Cooper pairs no longer contribute to heat transport and the thermal conductivity is much lower than in the normal conducting state. The severe decrease of the thermal conductivity of indium in its superconducting (sc) state is presented in figure 2.5 .

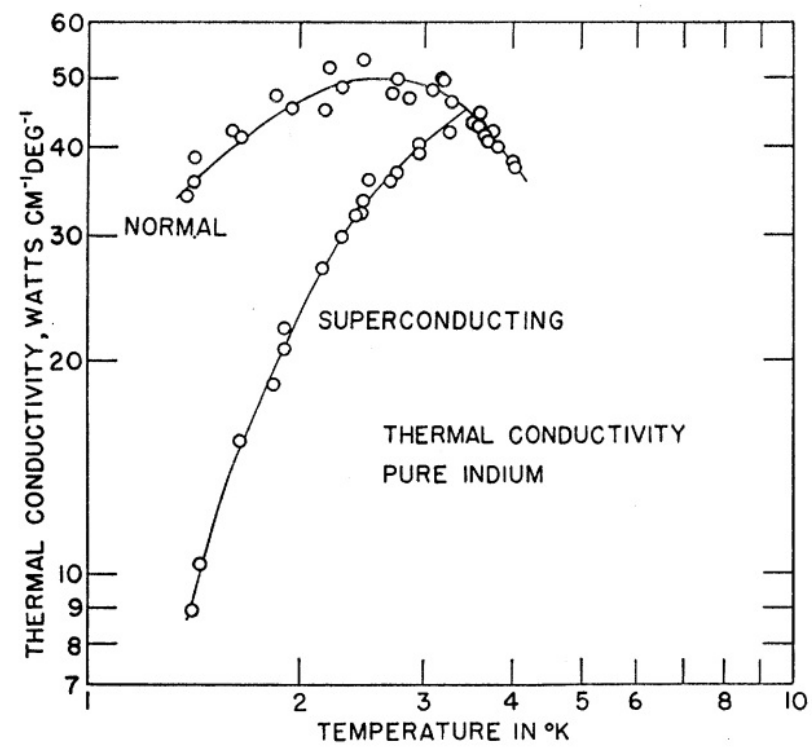

Figure 2.5: Thermal conductivity of indium in normal and superconducting states. It should be underlined that the presented thermal conductivity was measured on a sample of $R R R \approx$ 11000 [42]. The low temperature thermal conductivity of a lower purity sample would be significantly lower.

Superconductors are characterized by the existence of a critical temperature $T_{c}$ below which they lose their electrical resistance, if an external magnetic field strength is not higher than $H>H_{c}$ in the material (see figure 2.6a). A material in the superconducting state is perfectly diamagnetic. It generates an internal current in its outer shell such, that the corresponding magnetic field repels the external magnetic flux. The external flux enters only that outer layer of a superconductor, where it decays exponentially over a distance called 


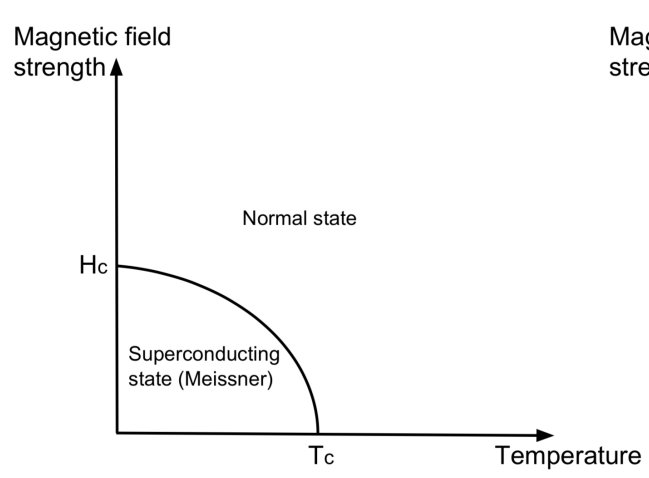

Type-I superconductivity

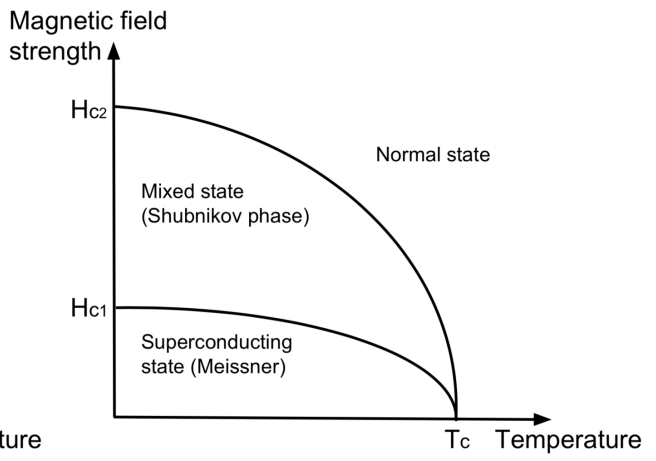

Type- II superconductivity

Figure 2.6: A comparison between type I and type II superconductors, exibiting correspondingly the Meissner effect (left), or the Meissner effect and Shubnikov phase, depending on the temperature and magnetic field strength (right). Picture copied from 43 .

"penetration depth". Theoretically, pure (without imperfections) type I superconductors show a perfect Meissner effect, i.e. they repel all of the external magnetic flux as shown in figure 2.7. In type II superconductors above a certain value of magnetic field $H_{c 1}$ a quantized amount of the flux traverses the material in the form of vortexes, pinning the magnetic field inside the superconductor, if pinning centers in the form of impurities or lattice imperfections are present (figure 2.8] [31. The surface occupied by the vortex grows with the increase of external magnetic field, until at a field strength $H_{c 2}$ the material loses its superconducting properties (figure 2.6p). It is important to mention, that $H_{c 2}>>H_{c 1}$, e.g. for $\mathrm{Nb}_{3} \mathrm{Sn} H_{c 1} \approx 40 \mu \mathrm{T}$ and $H_{c 2} \approx 23 \mathrm{~T}$. The pinning allows to generate very high magnetic fields by means of type II superconductors.

B

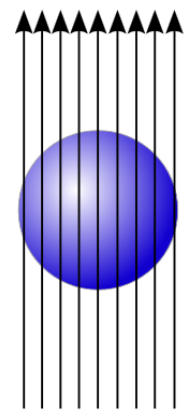

$\mathrm{T}>\mathrm{T}_{\mathrm{c}}$
B

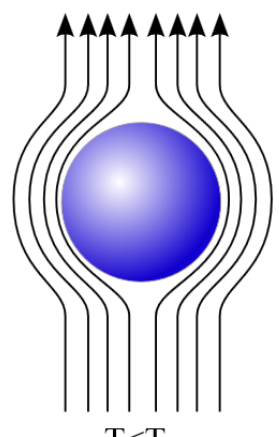

$\mathrm{T}<\mathrm{T}_{\mathrm{c}}$

Figure 2.7: Meissner effect in type I superconductor. All the external magnetic flux is repelled from the superconductor 43,44 . 
Pure elements are usually type I superconductors ( $\mathrm{Al}, \mathrm{In}, \mathrm{Nb}, \mathrm{Ti}, \mathrm{Pb})$, and alloys like NbTi type II superconductors. There exist also High Temperature Superconductors (HTS), usually much more complex compounds, like $\mathrm{YBa}_{2} \mathrm{Cu}_{3} \mathrm{O}_{7}$, that become superconducting at relatively high temperatures $\left(T_{c}\right.$ around $90 \mathrm{~K}$ ).

Interestingly, the orientation of a thin superconducting film versus the outer magnetic field may cause some additional effects. Because of the Meissner effect the density of flux lines just outside of the superconductor is higher than far away in a homogeneous field (see figure 2.7). Therefore in a thin-film superconducting flat disk oriented perpendicularly to the magnetic flux lines, the outer edge of the disk is subjected to much higher magnetic field than the nominal one. With increasing magnetic flux, the disk will lose its superconducting properties starting from the outer edge and propagating to the middle of the sample. This effect was observed for respective indium layers in the setup in the Cryolab DR and described by T. Eisel in his PhD thesis [4]. A superconducting thin film, thinner than the penetration depth, placed in a parallel magnetic field, may remain superconducting even for quite high magnetic field strengths [30]. Therefore, in multilayer setups including superconducting thin films one should take into consideration the shape and orientation of the thin film with respect to the external magnetic field. Both of the sandwich setups studied in the Cryolab DR, as well as the sapphire electrode in the AEgIS experiment contain thin layers of superconducting materials, subjected to the influence of an external magnetic field that can highly diminish their thermal performance.

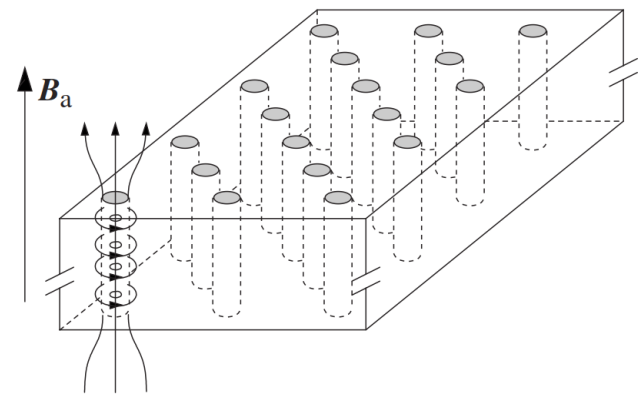

Figure 2.8: Quantised amounts of magnetic flux in a form of vortexes penetrating the type II superconductor in the Shubnikov phase 31.

\subsection{Thermal boundary resistance}

The main part of the Cryolab DR measurement campaigns focuses on the steady-state behaviour of the sandwich setup. Determining the main constraints of the heat flow, i.e. the thermal resistivity of the sandwich setup, 
is essential for the final design of the AEgIS electrode. Several models describing thermal boundary resistivity have been developed and are well known in literature. The most significant study of low-temperature thermal boundary resistances, that later influenced almost all of the modern models, was carried out by Pyotr Kapitza in 1941, who investigated the temperature discontinuity between liquid helium and a bulk copper body [45]. He assumed that the heat can only be transferred by phonons, and because of the high dissimilarity of materials only a limited amount of phonons can be transferred. According to the model the thermal boundary resistance, which is defined as:

$$
R=\frac{\Delta T}{\dot{Q}},
$$

has a $T^{-3}$ temperature dependence. Based on the work of Kapitza, further models were developed, that described the thermal boundary resistance between solids and included a wide range of modifications, taking into account more and more parameters. The models that are most important for the studied electrode application are described hereafter.

\subsubsection{Acoustic Mismatch Model}

Further analysis of the boundary resistance was conducted by I. M. Khalatnikov in 1952 [46] and in 1959 by W. A. Little, who described the acoustic mismatch model [47], which is an extension of the Kapitza thermal resistance theory to interfaces between solids out of which at least one is a dielectric. Phonons, being the only heat carriers in such a configuration, are treated as plane waves and the dielectric as an elastic continuum. Approaching the interface, waves can get reflected or transmitted, depending on the angle of incidence. By analogy to optics, Snell's law is used to define the critical angle that allows the transmission of phonons:

$$
\frac{\sin \alpha_{1}}{\sin \alpha_{2}}=\frac{v_{1}}{v_{2}}
$$

where $\alpha_{1}$ and $\alpha_{2}$ are the angles of incidence in material 1 and 2 , and $v_{1}$ and $v_{2}$ are the corresponding speeds of sound. Let's assume that material number 1 is more rigid, and therefore acoustically "better", i.e. $v_{1}>v_{2}$. In the interfaces sapphire - indium and sapphire - titanium, the metals are softer than sapphire. Approaching the respective interface from the metal side, the critical angle equals to:

$$
\alpha_{\text {crit }}=\arcsin \left(\frac{v_{\text {metal }}}{v_{\text {sapphire }}}\right)
$$

For the particular case of Kapitza resistance between liquid helium and copper the critical angle equals to only $3^{\circ}$, and for the configuration sapphire - indium to around $17^{\circ}$. The fraction of phonons falling in the critical cone for the 
sapphire - indium case is around $4.5 \%$. Moreover the transmission is further limited by the difference of acoustic impedances $Z=v \rho$, where $v$ is the speed of sound, and $\rho$ the density. The probability of transmission equals to 38 :

$$
t^{\mathrm{AMM}}=\frac{4 Z_{1} Z_{2}}{\left(Z_{1}+Z_{2}\right)^{2}}
$$

The rate of heat flow $\dot{Q}$ carried by phonons impinging the contact area $A$ from side 1 is [38]:

$$
\frac{\dot{Q}}{A}=\frac{\pi^{2} k_{B}^{4} \rho_{1} v_{1} T^{4}}{30 \hbar^{3} \rho_{2} v_{2}^{3}} .
$$

The resulting boundary resistance for $\Delta T \ll T$ is given as [38]:

$$
R=\frac{\Delta T}{\dot{Q}}=\frac{15 \hbar^{3} \rho_{1} v_{1}^{3}}{2 \pi^{2} k_{\mathrm{B}}^{4} \rho_{2} v_{2} A T^{3}} .
$$

For later analysis we define a thermal interface resistivity $(\Delta T \ll T)$ as:

$$
\kappa_{\text {interface }}=\frac{A T^{3} \Delta T}{\dot{Q}} .
$$

Material properties used to estimate the thermal resistivity are summed up in table 2.1. The more dissimilar the materials, the better the AMM predicts the interface resistance. Table 2.2 shows the values of the critical angle, transmission probability and boundary resistance for certain configurations of materials present in the setup simulating the ultra-cold electrodes. It is discussable whether one should take into account longitudinal $(\mathrm{L})$ or transverse $(\mathrm{T})$ values of the speed of sound. Here, both values are given for comparison and a further discussion will be carried out in chapter 5 .

Table 2.1: Material properties used to calculate the resistivity according to the acoustic mismatch model (L - longitudinal, T - transverse).

\begin{tabular}{|c|c|c|c|c|c|}
\hline Material & \multicolumn{2}{|c|}{ Speed of sound $(\mathrm{m} / \mathrm{s})$} & Density & \multicolumn{2}{c|}{ Acoustic impedance } \\
& $\mathrm{L}$ & $\mathrm{T}$ & $\left(\mathrm{kg} / \mathrm{m}^{3}\right)$ & $\mathrm{L}$ & $\mathrm{T}$ \\
\hline Sapphire 21] & 10800 & 6400 & 4000 & $4.32 \cdot 10^{7}$ & $2.56 \cdot 10^{7}$ \\
Indium 21] & 2600 & 1500 & 7470 & $1.94 \cdot 10^{7}$ & $1.12 \cdot 10^{7}$ \\
Titanium & 6070 & 3125 & 4510 & $2.74 \cdot 10^{7}$ & $1.41 \cdot 10^{7}$ \\
48.49] & & & & & \\
Gold 48 & 3240 & 1200 & 19300 & $6.25 \cdot 10^{7}$ & $2.32 \cdot 10^{7}$ \\
Copper 50 & 4600 & 2200 & 8960 & $4.12 \cdot 10^{7}$ & $1.97 \cdot 10^{7}$ \\
\hline
\end{tabular}

W.A. Little 47 theoretically considered in his work also the influence of many other parameters on the boundary resistance. He predicted that the surface quality may have a significant influence on resistivity, i.e. that the effect 
Table 2.2: Critical angle, probability of transmission and thermal boundary resistivity for various combinations of materials according to the AMM. Material properties used for the calculation of the thermal resistivity are taken from table 2.1 ( $\mathrm{L}$ - longitudinal, $\mathrm{T}$ - transverse).

\begin{tabular}{|c|c|c|c|c|c|c|}
\hline Interface & \multicolumn{2}{|c|}{ Critical cone } & \multicolumn{2}{|c|}{ Prob. of transm. } & \multicolumn{2}{|c|}{ Resistivity $\left.\frac{\mathrm{cm}^{2} \mathrm{~K}^{4}}{\mathrm{~W}}\right)$} \\
& $\mathrm{L}$ & $\mathrm{T}$ & $\mathrm{L}$ & $\mathrm{T}$ & $\mathrm{L}$ & $\mathrm{T}$ \\
\hline sapphire - & $14^{\circ}$ & $14^{\circ}$ & 0.86 & 0.85 & 63.65 & 22.96 \\
indium & $34^{\circ}$ & $43^{\circ}$ & 0.87 & 0.92 & 11.02 & 2.09 \\
copper - indium & $53^{\circ}$ & $53^{\circ}$ & 0.72 & 0.88 & 8.29 & 0.73 \\
gold - indium & $32^{\circ}$ & $23^{\circ}$ & 0.85 & 0.94 & 5.89 & 1.46 \\
titanium - gold & $34^{\circ}$ & $29^{\circ}$ & 0.95 & 0.92 & 45.20 & 18.27 \\
sapphire - & 3 & & & & & \\
titanium & & &
\end{tabular}

of surface roughness would depend on the phonon wavelength and that the application of a compression force could improve the contact and therefore decrease the thermal boundary resistivity. He indicated a path of further research concerning the possible couplings between electrons and surface waves, stating that the boundary resistance should change when the metal switches between normal and superconducting states. That information was the starting point of a research conducted by Papk and Narahara [51], discussed in the next section.

\subsubsection{Electron coupling to surface waves}

The possible coupling options between conduction electrons and surface waves were investigated by the Japanese researchers Papk and Narahara 51] and published in 1970, almost 10 years after Little. They developed a mathematical description of the phenomenon, in two cases: longitudinal waves approaching the interface, getting reflected and transmitted as separate longitudinal and transverse waves; and transverse waves approaching the interface getting also reflected and transmitted as longitudinal and transverse waves. They obtained an equation in the form of a Rayleigh surface wave, describing the interaction of waves with electrons. In both cases (longitudinal and transversal), the thermal conductivity was obtained in a solution that was consisting of two components: with $T^{3}$ dependence - as in a classic Kapitza resistance case (AMM), and a component scaling with $T^{5}$ representing the coupling of electrons to surface waves, see equation (2.23). It should be underlined, that this contribution to the thermal conductivity is only present if electrons are available on the acoustically better side, characterized by a higher speed of sound. This contribution is therefore not present at a nc indium - sapphire interface, but could be present at a sc indium - copper interface. A numerical evaluation of the formula presented by Papk, assuming solids with Poisson's ratio of $1 / 3$, equal density of materials and the speed of sound in a metal 3 times higer than in the dielectric results 
in the following thermal conductivity (defined simply as $\dot{Q} / \Delta T)[51$ :

$$
\frac{\dot{Q}}{\Delta T}=\alpha T^{3}+\beta T^{5}
$$

where $\alpha=4 \cdot 10^{3} \mathrm{~W} / \mathrm{K}^{4}$ and $\beta=0.5 \mathrm{~W} / \mathrm{K}^{6}$ for the longitudinal waves, and $\alpha=13 \cdot 10^{3} \mathrm{~W} / \mathrm{K}^{4}$ and $\beta=7 \mathrm{~W} / \mathrm{K}^{6}$ for the transverse waves. In equation (2.23) the prefactor $\beta$ is three to four orders of magnitude lower than $\alpha$, so the influence of the $T^{5}$ component is predicted to be small.

Papk and Narahara experimentally verified that a change of boundary resistance between sapphire and indium exists, when indium changes form the normal to the superconducting state, i.e. when the valence electrons that could couple to eventual surface waves become unavailable. Since the speed of sound in sapphire is much higher than in indium, according to their theory, there should be no difference caused by couplings of electrons. They have measured the sapphire - indium boundary thermal resistivity in a temperature range from $0.6 \mathrm{~K}$ to $2.2 \mathrm{~K}$ and contrary to their expectations, recorded a small difference of resistivities in normal and superconducting states $\frac{R_{\mathrm{s}}}{R_{\mathrm{n}}} \approx 1.05$. One could imagine that a measurement of an interface between materials actually fulfilling the speed of sound condition $v_{\text {metal }}>v_{\text {dielectric }}$ could show a much higher contribution of the surface waves to the thermal conductance.

\subsubsection{Diffuse Mismatch Model}

The diffuse mismatch model (DMM) was developed by E.T. Swartz in his PhD thesis in 1987 [52. In contrast to the AMM, it assumes that absolutely no phonons are specularly reflected at the interface. Phonons can only scatter forward (get transmitted) or scattered back, but in both cases they "forget" where they came from - there is no angular dependence between the incident and outgoing phonons. All the collisions are elastic, which means that a phonon can only forward its energy to another phonon of the same frequency. The probability of transmission in the DMM $t^{\mathrm{DMM}}$ depends on the speed of sound in materials:

$$
t^{\mathrm{DMM}}=\frac{\Sigma_{j} v_{2, j}^{-2}}{\left(\Sigma_{j} v_{1, j}^{-2}+\Sigma_{j} v_{2, j}^{-2}\right)}
$$

where $j$ denotes longitudinal and transverse modes. Phonon scattering at the interface can reduce the thermal boundary resistance for the case of very dissimilar solids. For such a case, the thermal resistance calculated with DMM would be much lower than for the AMM. The roughness of the surface with respect to the phonon wavelength is the main parameter determining whether the heat transfer across the interface will follow acoustic or rather diffusive mismatch model.

According to the AMM, a chance of transmission across a perfectly smooth interface between two exactly the same materials (dissimilarity $=0$ ) would be 


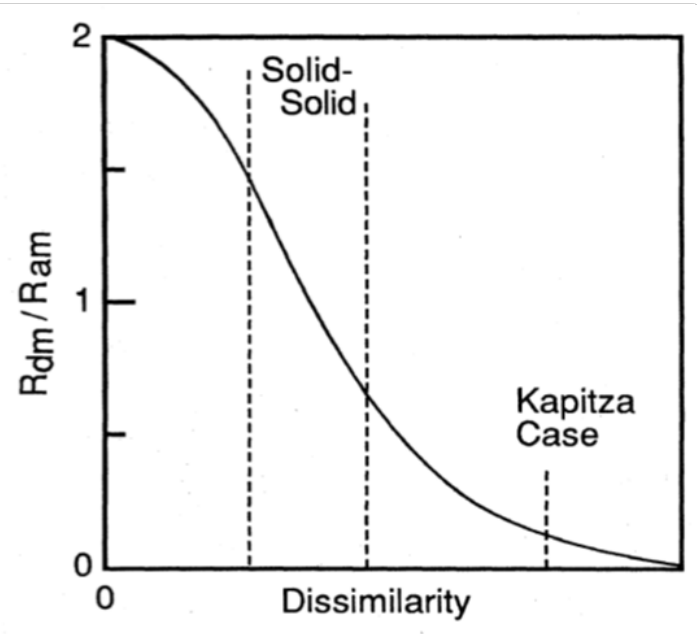

Figure 2.9: Ratio of the boundary resistance values predicted by the acoustic mismatch model and the diffuse mismatch model depending on the dissimilarity of materials from 53 .

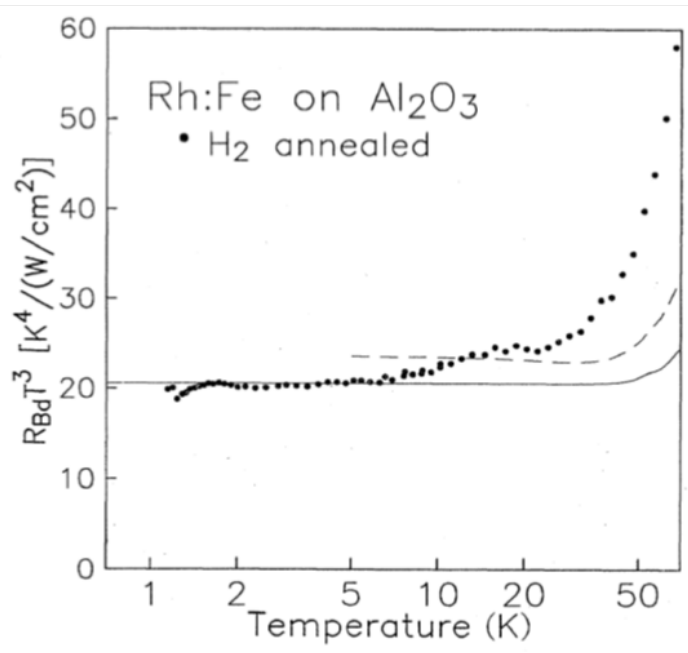

Figure 2.10: The transition between acoustic and diffuse regime of thermal boundary resistance visible at the example of an interface between iron-doped rhodium and sapphire. The continuous line is a prediction of the AMM, and the dashed one of the DMM. Black dots are measurement points. One can see that the measurement follows the AMM theory quite well up to around $7 \mathrm{~K}$, when the phonon wavelength becomes shorter, the phonons get stattered on the interface, and then a transition to the DMM occurs [52].

equal to $100 \%$. The DMM on the other hand, predicts $50 \%$ of a chance for transmission in this case, so the ratio $R_{\mathrm{dm}} / \mathrm{R}_{\mathrm{am}}=2$. The ratio of the resistance values predicted by the AMM and the DMM as a function of dissimilarity of materials is presented in figure 2.9 [53. The line described as "Kapitza case" is an example of a very dissimilar interface between copper and sapphire. 
The range described as "solid-solid" has a much lower dissimilarity, typically observed between two solid bodies.

E. T. Swartz managed to observe the transition between acoustic and diffuse regime in reality at the example of the interface between iron-doped rhodium and sapphire. The plot of measured thermal boundary resistance of $\mathrm{Rh}: \mathrm{Fe}$ on sapphire versus temperature is shown in figure 2.10. For low temperatures the phonon wavelength is longer than the roughness of the interface, and the resistance follows very closely the AMM. At approx. $7 \mathrm{~K}$ the wavelength becomes comparable or shorter than the roughness of the surface, the phonons are getting scattered, and the behaviour starts following the prediction of the DMM.

Other interesting attempts to estimate the thermal boundary resistance, mainly at higher temperatures, are described in the next section.

\subsubsection{Advanced higher temperature models}

\section{Joint Frequency Diffuse Mismatch Model}

The Joint Frequency Diffuse Mismatch Model (JFDMM) is a modification of the Diffuse Mismatch Model described by Hopkins and Norris in 2007 [54]. It is meant to deal with temperatures of an order of a hundred Kelvin (or above the Debye temperature), where the DMM fails to correctly predict the boundary resistance. The JFDDM assumes that the phonons close to the boundary, i.e. within the mean free path from the interface, vibrate with a frequency $\omega_{\text {mod, } j}$, which is an average phonon frequency of both interface sides, with weight factors $\xi_{i}$ depending on the atomic masses $M_{i}$ and the number of states $N_{i}$ available on each side. Such frequency is definitely higher than the phonon frequency on the acoustically "weaker" side (with lower speed of sound) and therefore enables more phonons to get transferred. This approach is rather easy to implement mathematically, and therefore it could be considered as a convenient method of decreasing the modelled thermal boundary resistance of the sandwich setup.

\section{Multiphonon processes}

All of the models discussed so far assume that only two phonons participate in the collisions responsible for the interfacial heat transfer. In reality however, it could happen that three or even more phonons can collide at once, or one phonon can decay into two, and significantly change the heat transfer coefficient. Inelastic multi-phonon processes were investigated and described by P. Hopkins [55]. His study considers much higher temperatures than those of our interest, but perhaps some of the described mechanisms are present also in the ultra-low temperature range, and therefore could be used in the modelling of the Cryolab DR sandwich setup. 
This model predicts that two phonon processes are more probable than three phonon processes, three phonon more probable than four, etc. The contribution of each type of a process according to this model at an example of a $\mathrm{Pb} /$ diamond interface is presented in figure 2.11. The thermal resistivities of not all the interfaces are predicted equally successfully with use of this model as the presented example of $\mathrm{Pb} /$ diamond, but one can clearly see that including three phonon processes in the modelling is highly justified for high temperatures.

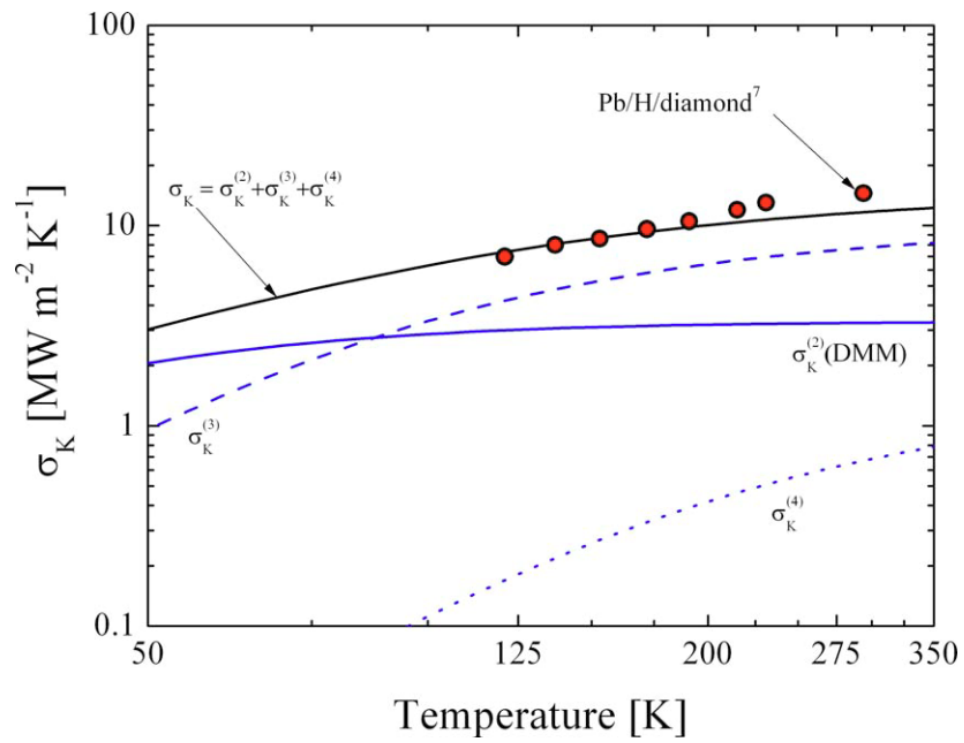

Figure 2.11: "High" temperature thermal boundary conductance $\sigma_{K}$ predicted by the multiphonon model of $\mathrm{P}$. Hopkins copied from [55] at a $\mathrm{Pb} /$ diamond interface. The contribution of each type of a process is marked $\left(\sigma_{K}^{(n)}-\mathrm{n}\right.$-phonon). $\sigma^{(\mathrm{DMM})}$ is a prediction of the thermal boundary resistance calculated with the DMM.

\section{Machine learning}

The latest attempt of handling the problem of the unknown thermal boundary resistance involves one of the newest disciplines of science - machine learning. In a scientific report published in Nature in 2017, Zhan, Fang and Xu [56] described how by training the neuron network with multiple examples taken from 62 literature sources, they succeed in predicting the value of thermal boundary resistance, obtaing a corelation between predicted and experimental values even higher than those of pure AMM and DMM. It should be underlined however, that the researchers focused on rather high temperature applications, which is not what AMM or even DMM were intended for. The result depends heavily on the set of parameters used for training, and on the applied training 
algorithm, but in general it can be considered as a very interesting and successful approach. Figure 2.12 presents a correlation between the experimental values, and values predicted with a Gaussian Process Regression (GPR) model obtained by the Japanese authors.

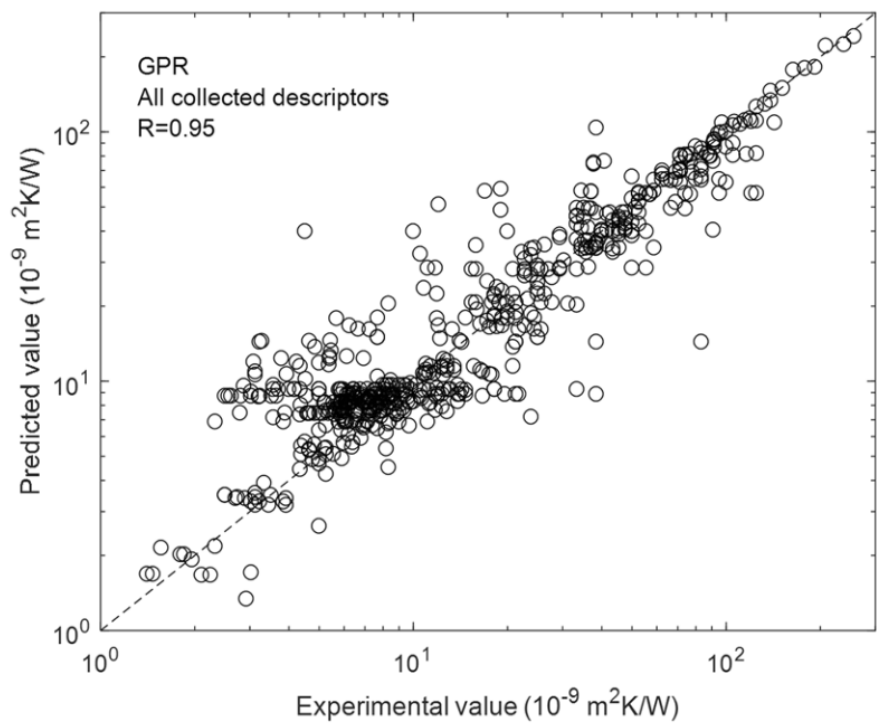

Figure 2.12: Correlation of experimental values of thermal boundary resistance and values predicted with a GPR model, obtained with a machine learning method 56.

Not too many models predicting the very low temperature interface resistance exist. For ultra-low temperatures, when the wavelengths of the heat carriers are long, the AMM remains the most reliable and theory based description of the phonon behaviour at the interfaces. Therefore, despite its disadvantage of frequent overestimation of the value of the interface resistivity, it will be used as a base for the mathematical model of the sandwich setup.

\subsection{Thermal conductivity of thin layers}

Many researchers have already described the fact that the thermal conductivity of a thin layer differs significantly from that of a bulk material [57 62], being also significantly different across and along the thin layer [57,59]. The problem is usually referred to, in a context of nano-layers in microelectronics, when the mean free path of heat carriers is longer than the thickness of a layer, even at temperatures way higher than the milli-Kelvin range. In thin layers of high temperature superconductors, a reduction of the film thickness leads to a decrease of its thermal conductivity as reported by Flik and Tien [59. Their plot of the ratio of the thermal conductivity across the layer to the conductivity 
along the thin layer as a function of the reduced film thickness is presented in figure 2.13. Assuming that the thermal conductivity can be still expressed as in eq. $(2.9)$, only the mean free path changes significantly with the thickness reduction. According to their method one can calculate the ratio of mean free path across the thin layer to the mean free path in a bulk material as:

$$
\frac{l_{\mathrm{eff}}}{l}=\frac{d}{l}\left(1-\frac{1}{2} \frac{d}{l}\right)
$$

which for an exemplary ratio of the film thickness $d$ to the mean free path $l$ : $d / l=0.1$, gives a reduction of the thermal conductivity by a factor of 0.095 .

Starting from slightly different assumptions, Flik obtained also a formula for the reduction of the mean free path in the form:

$$
\frac{l_{\mathrm{eff}}}{l}=\frac{1}{2} \frac{d}{l}\left(1+\exp \left(-m \frac{d}{l}\right)\right)-\frac{1}{6}\left(\frac{d}{l}\right)^{2},
$$

where $m$ is the matching parameter, which makes the solutions for the thermal conductivity across and along the film merge for $d=l$ when the value of $m$ is set to 6 . Then for the film thickness to the mean free path ratio $d / l=0.1$ and $m=6$ the reduction of thermal conductivity is even more severe than obtained from equation 2.25 and equals to $k_{\mathrm{eff}} / k=0.076$.

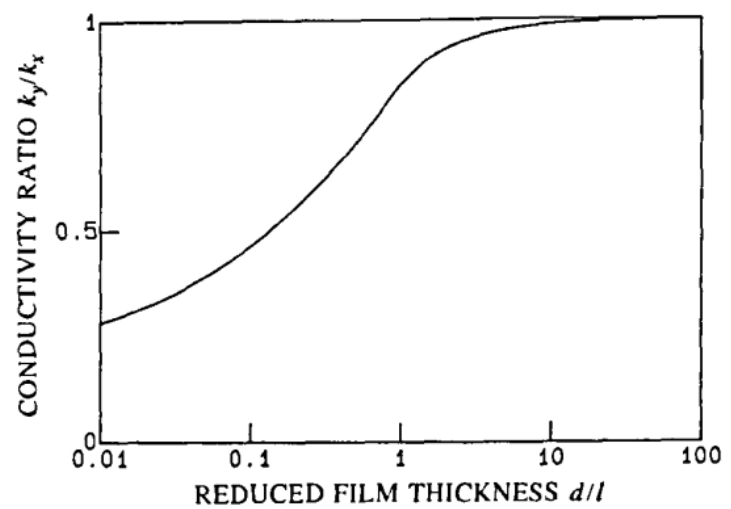

Figure 2.13: The ratio of thermal conductivities across and along the thin film $k_{y} / k_{x}$ as a function of the reduced film thickness $d / l$, where $d$ is the film thickness, and $l$ is the mean free path of heat carriers from 59.

Another approach presented by Majumdar [63] suggests that the effective mean free path should be calculated with the formula:

$$
\frac{l_{\mathrm{eff}}}{l}=\frac{1}{1+\frac{4 l}{3 d}}
$$


which for the same ratio $d / l=0.1$ gives a reduction of the thermal conductivity of an order of $k_{\text {eff }} / k=0.075$, which is almost the same as the result obtained with eq. $(2.26)$.

Heino used the molecular dynamics method 61 to simulate the behaviour of thin films, and reported that for very thin films with a thickness in the order of several nanometers, the phonon group velocity and the dispersion relation changes.

Turney, McGaughey, Amon [60], who based their research on lattice dynamic calculations, stated that for very thin films also the phonon density of states is different than in a bulk material. The reduction of the thermal conductivity obtained by Turney with various methods as a function of the film thickness, is presented in figure 2.14

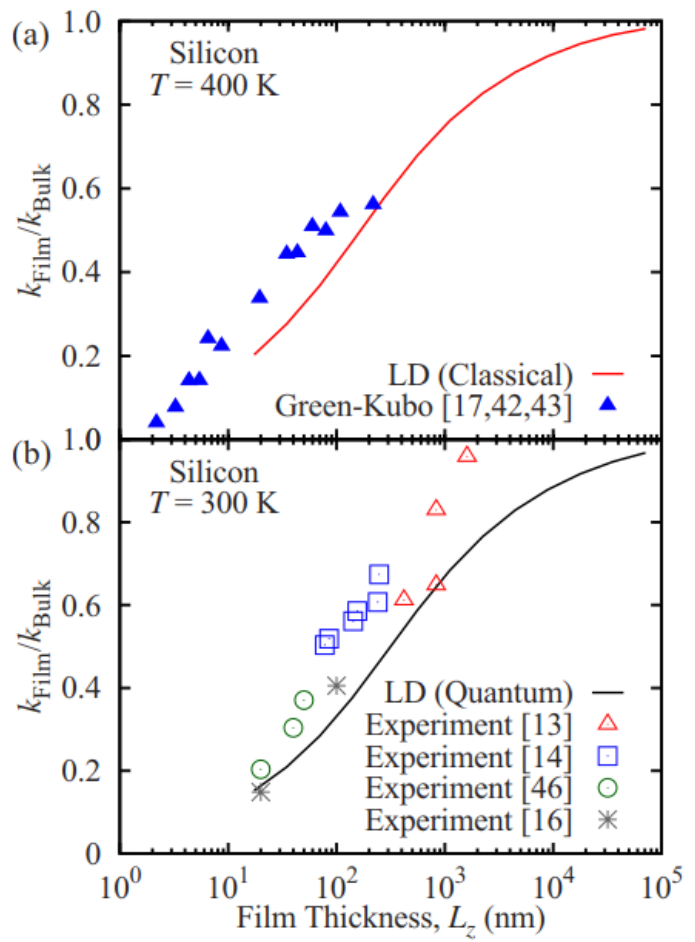

Figure 2.14: The reduction of the thermal conductivity for a silicon thin film obtained by Turney from: a) classical lattice dynamics (LD) calculations and the Green-Kubo method; b) quantum lattice dynamics calculations and the experiment 60 .

Langer, Hartmann and Reichling [62] measured the thermal conductivity of thin films of gold and nickel on a quartz substrate with the modulated thermoreflectance method. They stated that the thermal conductivity of gold depends not only on the thickness of the film, but also on the polycrystalline structure of it, due to the sputtering process. The thermal conductivity of the thin films of gold and nickel as a function of the layer thickness is presented in 
figure 2.15. As one can see, the thermal conductivity of gold highly depends on the thickness of the layer. The conductivity of nickel is much lower than gold at high temperatures, and it does not decrease significantly with a decrease of temperature. Moreover, they pointed out that the phonon contribution to the conductivity of thin metallic films may be significant due to lattice imperfections. Such conclusion could be an important information used in the mathematical model of the sandwich setup.

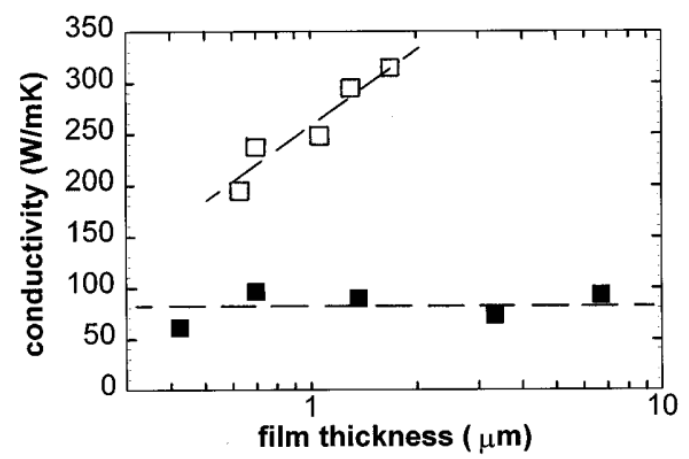

Figure 2.15: Thermal conductivity of gold (empty squares) and nickel (full squares) as a function of the film thickness from 62 . Dashed lines are guides to the eye.

One more effect that could be observed in perfect crystals of dielectrics or metals is the multiple reflections at outer boundaries. If the ratio of the mean free path of a phonon in a material to the thickness of the layer itself are comparable, or if the mean free path is larger than the thickness of the material, one speaks about the so called ballistic regime [64]. In such case the phonons propagate freely across the material without scattering until they reach the other side of the sample, where they can get transmitted, reflected or scattered. In this situation, the effective thermal conductivity of the thin layer and its interfaces depends on the ratio of the phonon wavelength to the roughness of the sample outer surfaces, where it can behave as described by the AMM or DMM. If the wavelength is definitely longer than the surface roughness and the angle of incidence lies within the cone of acceptance, there is a chance for transmission, see eq. 2.18) and 2.19). In a configuration of two parallel, but not perfectly parallel planes, also phonons approaching from slightly outside of the cone, bouncing at the interfaces several times (see figure 2.16), may change their angle of incidence after multiple reflections and eventually get transmitted, what can increase the effective conductivity. If the phonon wavelength is shorter than the roughness of the surface, the thermal conductivity of the interface follows the DMM, the phonons get scattered, and the parallel plates assumption is not valid.

Flik [59], Heino [61], Turney [60] and Langer [62] were considering the behaviour of thin films and its dependence on the mean free path of the heat 


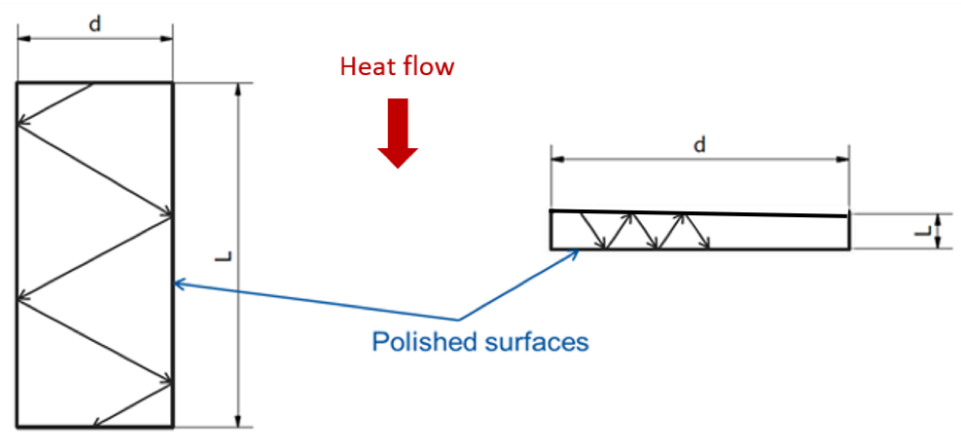

Figure 2.16: Schematics of two configurations in which ballistic transport of phonons may increase the effective thermal conductivity.

carriers mainly at high temperatures. One could expect that the same results would be observed even in much thicker films at ultra-low temperatures. However, when the temperature decreases to several milli-Kelvin, one more severe constraint appears: the phonon wavelength itself can reach or even exceed the thickness of a thin layer. The question remains how much the effective conductivity decreases in such a situation. Could all the atomic layers in the thin film move all together in parallel? It remains unsure, whether valence electrons in such a thin layer could interact with eventual surface waves and significantly influence the effective thermal conductivity.

Wang 65] conducted a computational study of a phonon transport in $\mathrm{Si}$ thin films. He concluded that the phonon spectrum is confined, as the phonon wavelength has to fit in the thickness of the layer. The reduction of the thermal conductivity caused by the phonon confinement could reach even 3 orders of magnitude (see fig. 2.17). The temperature range of Wang's research was again - significantly higher than the range of this study, but surely the same effect of phonon confinement would be present at ultra-low temperatures in much thicker layers. Table 2.3 summarises the properties of the thin layers of the Cryolab DR sandwich setup and the temperatures at which the wavelengths of phonons in each layer reach a size comparable to the thickness of the layer itself. For the thin layer of titanium, the corresponding temperature is $5.8 \mathrm{~K}$. At approx. $0.4 \mathrm{~K}$, when the titanium becomes superconducting, the wavelength associated with the vibrations of phonons is much much longer than the thickness of the layer. Therefore, the thermal conductivity of the sandwich setup in the superconducting state may be severely affected by the exceptionally poor thermal performance of the thin layer of titanium in the whole investigated temperature range of $30-500 \mathrm{mK}$. 


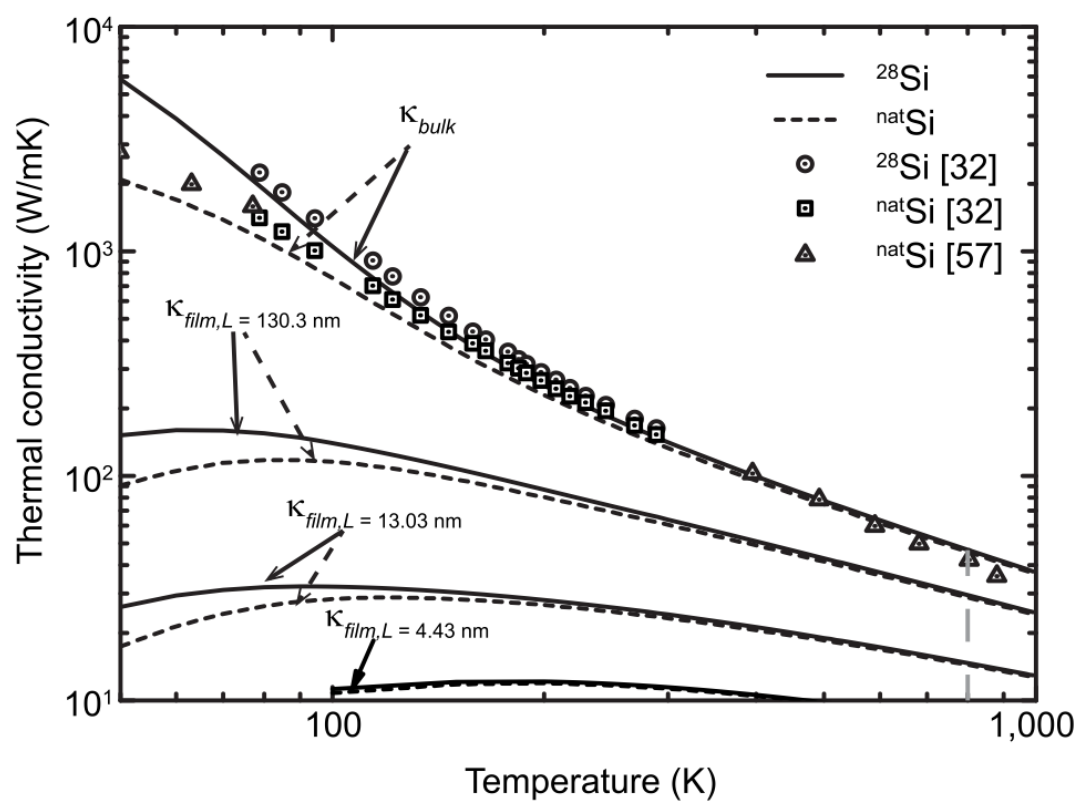

Figure 2.17: The thermal conductivity of Si samples as a function of temperature calculated by Wang 65. Samples of bulk Si and thin films of three different thicknesses $130.3 \mathrm{~nm}$, $13.03 \mathrm{~nm}$, and $4.34 \mathrm{~nm}$ were analysed. The solid line corresponds to results for isotope enriched samples, and the dashed ones for samples of natural isotopes. The circular, triangular and square points are experimental results 66 67.

Table 2.3: Temperatures at which the dominant wavelengths of phonons in thin layers of sapphire, indium, gold and titanium of the sandwich setup is comparable to their thicknesses. Longitudinal values of the speed of sound from table 2.1 were taken for this estimation.

\begin{tabular}{|c|c|c|c|}
\hline Material & Thickness & Speed of sound & Temperature \\
\hline Sapphire & $1 \mathrm{~mm}$ & $10800 \mathrm{~m} / \mathrm{s}$ & $0.52 \mathrm{mK}$ \\
Indium & $125 \mu \mathrm{m}$ & $2600 \mathrm{~m} / \mathrm{s}$ & $1 \mathrm{mK}$ \\
Gold & $750 \mathrm{~nm}$ & $3240 \mathrm{~m} / \mathrm{s}$ & $210 \mathrm{mK}$ \\
Titanium & $50 \mathrm{~nm}$ & $6070 \mathrm{~m} / \mathrm{s}$ & $5.8 \mathrm{~K}$ \\
\hline
\end{tabular}

\subsection{Thermal diffusivity}

Thermal diffusivity is a material property indicating how well the material transports heat as a function of time. It is expressed in the form of the Fourier equation:

$$
\frac{\partial T}{\partial t}-a \nabla^{2} T=0
$$


where $a$ is the thermal diffusivity in $\mathrm{m}^{2} / \mathrm{s}$. The value of the diffusivity can be calculated from other known material properties

$$
a=\frac{\lambda}{c \rho},
$$

where $\lambda$ is the thermal conductivity, $c$ heat capacity and $\rho$ is the density. The higher the value of the thermal diffusivity, the faster the material equalizes temperature after receiving a heat pulse, or builds a steady state temperature gradient in case of a boundary condition of constant but different temperatures at the two sides of the sample (in a 1D case).

As stated in section 1.2, the heat load in the AEgIS project will be arriving in very short pulses of $10^{-5} \mathrm{~J}$ every $100 \mathrm{~s}$. Therefore, not only the thermal conductivity of the sandwich setup, determined by multiple thin layers and interfaces, must be verified, but also its thermal diffusivity. The response to dynamic heat loads and the thermal anchoring of the electrodes should be efficient enough to make sure that the temperature of the electrodes does not exceed $100 \mathrm{mK}$, or in the worst case it decreases below $100 \mathrm{mK}$ as fast as possible after the heat pulse.

The thermal diffusivity measurement methods along with the evaluation of their validity are described in chapter 4 . 


\section{Chapter 3}

\section{Experimental setup}

The complex shape of the electrode does not allow to study the effects of the various interfaces and material properties separately. Hence, we use a mockup sandwich structure, that contains the same materials and interfaces as the electrode mounted on its thermalisation plate in the AEgIS. The mock-up is much easier to vary its parameters, and therefore it is more practical for studying the interface properties. The measurements were performed at ultralow temperatures, i.e. in the temperature range from $30 \mathrm{mK}$ to $500 \mathrm{mK}$, in the Cryolab Dilution Refrigerator and at low temperatures, i.e. in the temperature range from $3 \mathrm{~K}$ to $60 \mathrm{~K}$, on the Cryolab pulse tube cryocooler. This chapter presents a description of the two sandwich setups simulating the electrode and the devices used in the measurement campaigns.

\subsection{Mock-up of the electrode}

The performance of two types of the sandwich structure has been measured. Each of them allows us to investigate the properties of thin layers and various interfaces in the normal conducting and in the superconducting states. In both setups, the dielectric is in the form of a flat polished sapphire disk of $20 \mathrm{~mm}$ diameter and $1 \mathrm{~mm}$ thickness. As indicated by T. Eisel [4], a sandwich with an optically polished sapphire surface has much lower thermal resistance, than with a rough one [4], so only the polished sapphire was used.

\subsubsection{Copper - indium - sapphire sandwich}

In the first run of the Cryolab DR a sandwich setup that is schematically depicted in figure 3.1 was measured. It consisted of the following elements:

- stamp made of high conductivity oxygen free (OFHC) copper,

- $125 \mu \mathrm{m}$ thick indium foil, 
- $1 \mathrm{~mm}$ thick sapphire disk with flat, polished surfaces and a $2 \mu \mathrm{m}$ thick layer of vapour deposited indium on both flat sides,

- $125 \mu \mathrm{m}$ thick indium foil,

- platform made of OFHC copper.

Indium has a characteristic property of cold welding to itself and creates good mechanical connections to other metals, when left under pressure exceeding its yield limit for several days [5]. The surfaces of indium foil and copper were scratched mildly and rinsed with acetone and isopropanol just before mounting to minimize oxide layers. The clamping structure presented in figure 3.1 is the same as used by T. Eisel [4] in his measurements. It generates force high enough for indium to creep out and create a "cold weld". Moreover, it includes multiple thin layers of fiberglass-epoxy laminate, G10, to drastically reduce the parasitic heat escaping from the stamp to the platform via the clamping structure, bypassing the indium interfaces. To obtain a proper mechanical and thermal connection four M5 bolts were re-tightened with a torque $1.2 \mathrm{Nm}$ several times during a few days and spring washers were used to maintain the compression force as the indium creeped out. At the end the whole setup was placed on the copper lid of the mixing chamber of the dilution refrigerator, with a $125 \mu \mathrm{m}$ layer of indium in between to ensure proper thermal contact also between the platform and the lid. Separate layers of the sandwich and the whole setup are presented in figure 3.1 .

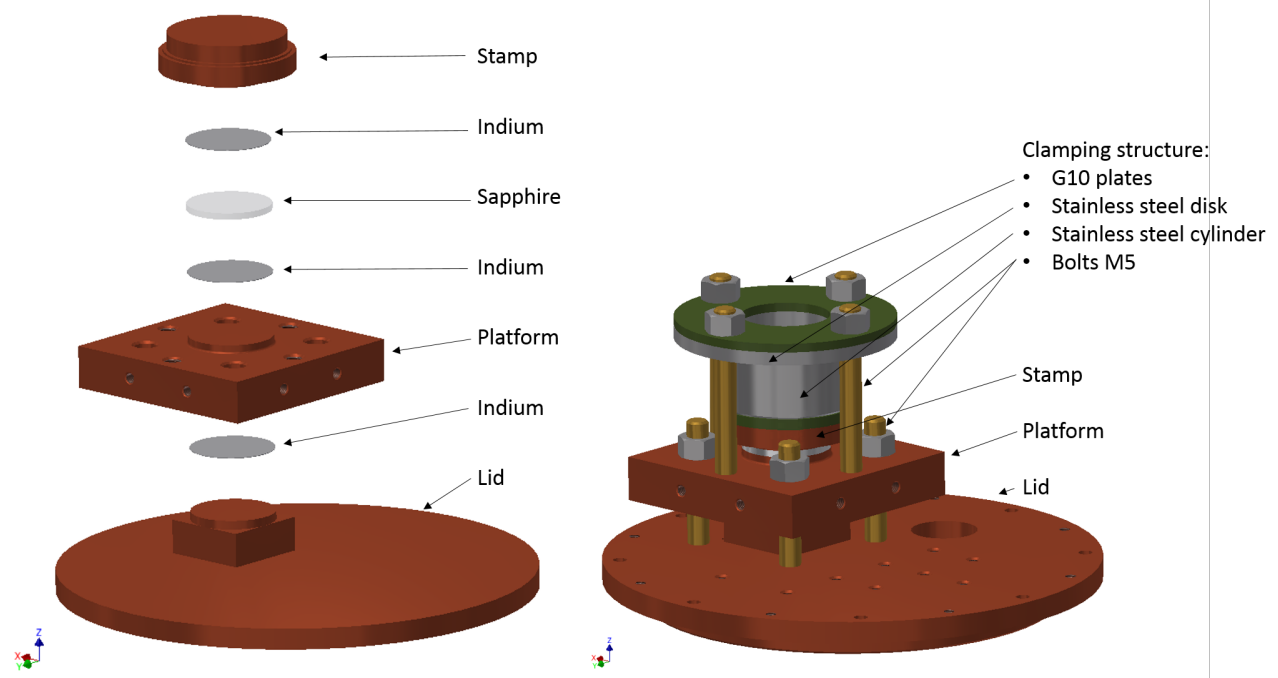

Figure 3.1: Left: layers of the sandwich from bottom to top: lid $(\mathrm{Cu})$ - indium foil - platform $(\mathrm{Cu})$ - indium - sapphire - indium - stamp $(\mathrm{Cu})$. Right: model of the setup on the mixing chamber lid. Thanks to the platform the setup is fully separable from the dilution refrigerator for the preparation and assembly. 
This setup was measured in the dilution refrigerator several times in different configurations: compressed, without compressing force, without the clamping structure and at the end in a wider temperature range. Indium becomes superconducting below $3.4 \mathrm{~K} \mathrm{[31]}$, but thanks to the presence of an external magnetic field, one can force it into the normal conducting state, and thereby significantly change the thermal conductivity of the sandwich. Without an external magnetic field, when the indium is superconducting and the valence electrons are not available for heat transfer, there are four interfaces involving dielectric-like behaviour: $\mathrm{Cu}$ - In, In - sapphire, sapphire - In and In - Cu. The application of an external magnetic field reduces it to two dielectric-like interfaces: In - sapphire and sapphire - In. The different thermal behaviour of each case will be presented in chapter 6 .

\subsubsection{Sandwich with $\mathrm{Ti}$ - Au coating on the sapphire}

The idea of creating the electrode by a deposition of gold on a sapphire base presents a certain technological challenge. The adhesion of gold to sapphire is not perfect and not all the methods can achieve the required results, especially taking into account the very complicated geometry of the full scale electrode. The electrode manufactured for G. Burghart with a sputtering technique had $45 \mathrm{~nm}$ of titanium below $520 \mathrm{~nm}$ of gold, where the Ti was necessary for the adherence. Therefore in the second sandwich all the layers are represented, as they were on the electrode, to investigate possible effects that might have decreased the overall conductivity. The second sandwich consists of the following elements:

- stamp made of OFHC copper,

- $125 \mu \mathrm{m}$ thick indium foil,

- $520 \mathrm{~nm}$ of sputtered gold,

- $45 \mathrm{~nm}$ of sputtered titanium,

- $1 \mathrm{~mm}$ thick sapphire disk with flat, polished surfaces,

- $45 \mathrm{~nm}$ of sputtered titanium,

- $520 \mathrm{~nm}$ of sputtered gold,

- $125 \mu \mathrm{m}$ thick indium foil,

- platform made of OFHC copper.

The sapphire disks with sputtered titanium and gold, still mounted in the deposition holder, are presented in figure 3.2. In this configuration there is not only one, but two materials with superconducting properties, as titanium 
becomes superconducting below $0.39 \mathrm{~K}$ [31], what makes the thermodynamic situation even more complex. The layers of titanium and gold are considerably thinner than the layer of indium in the first setup. The whole setup being compressed with four M5 bolts is shown in figure 3.2. Later on, it was mounted on the lid of the mixing chamber with a $125 \mu \mathrm{m}$ thick indium foil in between, and the clamping structure was removed.

The microstructure and elemental distribution of both sandwiches were verified and are described in the next section.
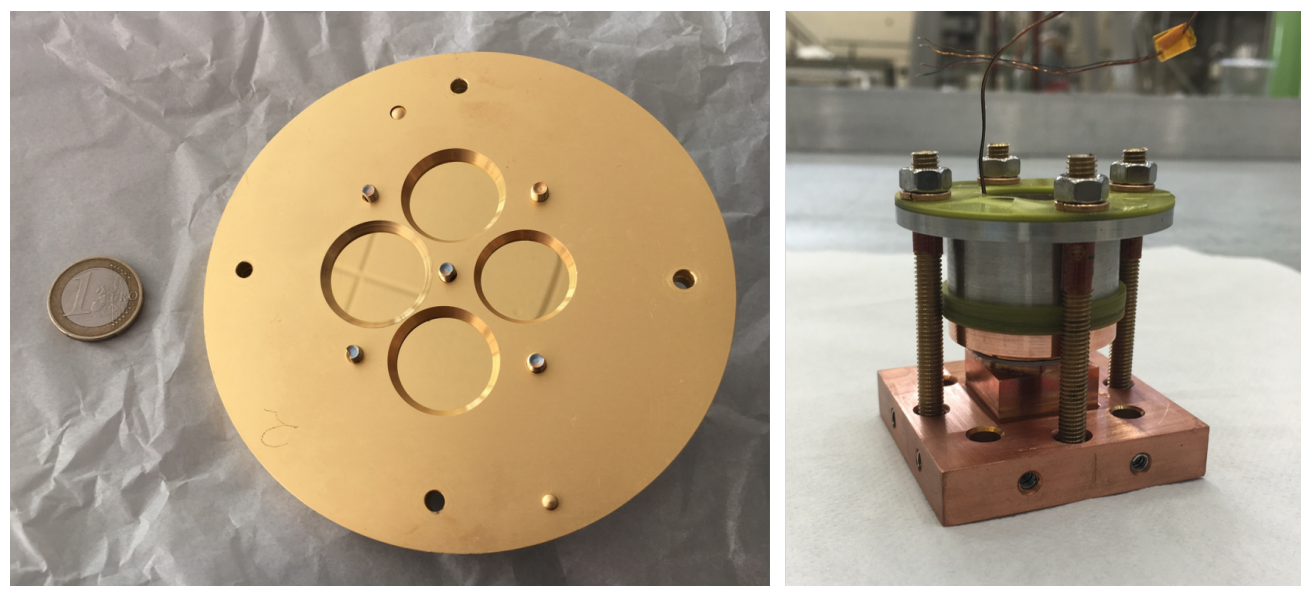

Figure 3.2: Left: Four sapphire disks with deposited $\mathrm{Ti}$ and $\mathrm{Au}$ in a holder for deposition; right: sandwich setup clamped with a force necessary for the indium to create a solid bond.

\subsubsection{Verification of microstructure and elemental distribution in sandwich thin films}

After all the measurement campaigns, the microstructure of both sandwich setups was checked with a Scanning Transmission Electron Microscopy (STEM) and the elemental distribution was verified with a Transmission Energy Dispersive X-ray Spectroscopy (TEDS) by Alexander Lunt, CERN EN-MME-MM [68]. Ultra thin lamellae were cut out of the sapphire disks with deposited thin films: indium in the first case, and titanium and gold in the second. The lamellae were cut with a Focused Ion Beam (FIB) method [68].

The STEM images of the thin layer of indium show little evidence of grain boundaries (figure 3.3), what suggests that it may be a single crystal. A single crystal structure would be very favourable for the heat transfer across the sandwich. The interface between sapphire and indium is sharp, what justifies the use of the AMM as an estimation of the boundary resistance. The thickness of the deposited indium layer equals to $2 \mu \mathrm{m}$, as expected. The line scan of atomic weight percent across the thickness of the film is shown in figure 3.4 . Before scanning, the sapphire - indium sandwich was covered with a layer 
of platinum, visible below $0.2 \mu \mathrm{m}$. Above $2.2 \mu \mathrm{m}$ the only constituents are aluminium and oxygen in sapphire $-\mathrm{Al}_{2} \mathrm{O}_{3}$. Certain oxidation of the outer surface of indium is also visible on the line scan around $0.2 \mu \mathrm{m}$, because that surface was exposed to atmosphere for over 1 year after the measurements in the Cryolab DR and before the STEM analysis.

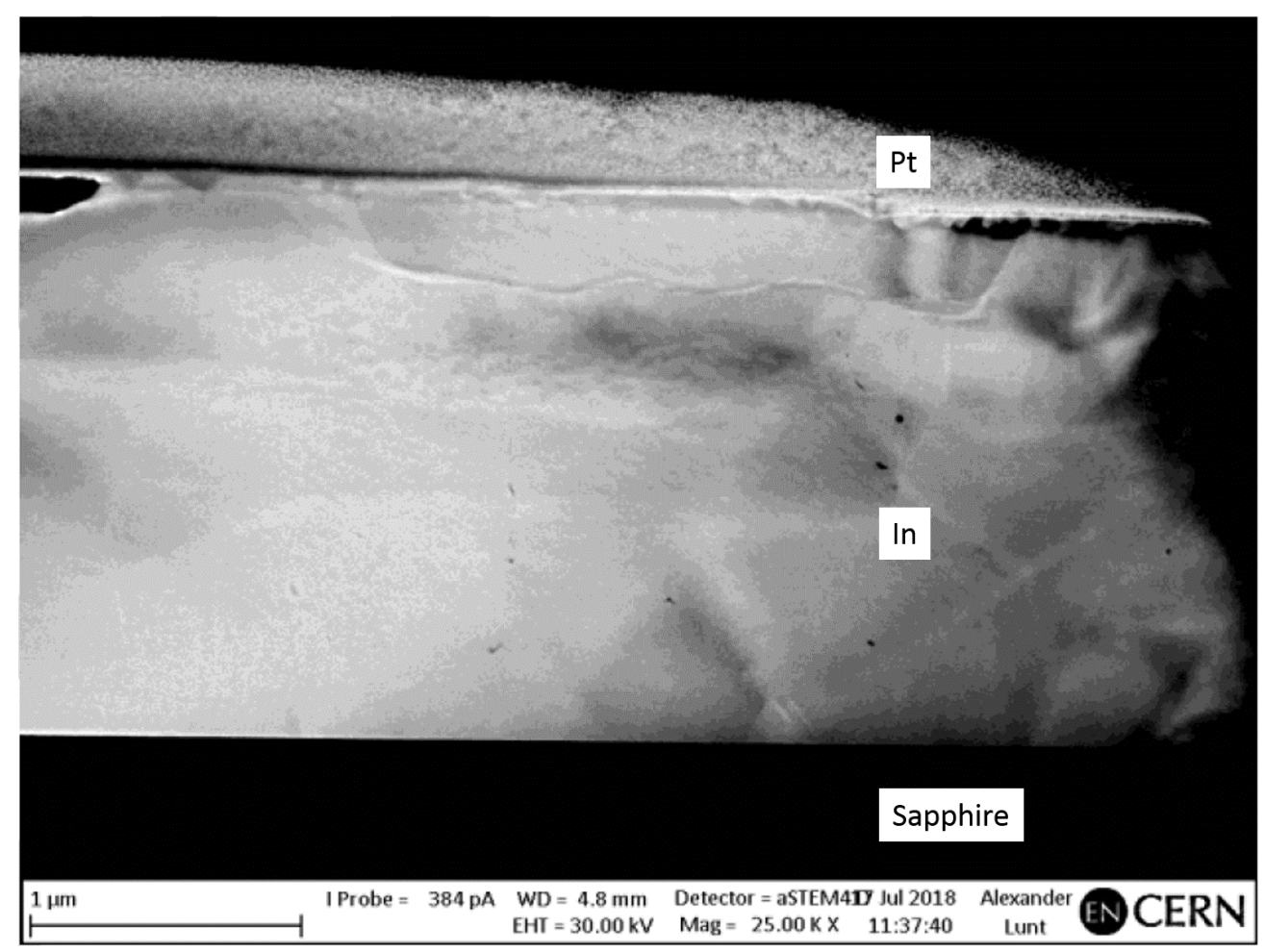

Figure 3.3: STEM image of the $2 \mu \mathrm{m}$ layer of indium on a sapphire substrate. No grains in the thin layer are visible, what indicates that indium may be a single crystal. A sharp sapphire - indium interface is visible.

The STEM images of the second sandwich (sapphire - Ti - Au) show a grainy, policrystalline structure of both titanium and gold (figure 3.5). As mentioned in literature [62], a structure of that type can significantly decrease the effective thermal conductivity of a thin film of gold. The top surface of the gold layer is not perfectly flat, and certain waves are visible. The thin layer of titanium has a distinct, sharp interface with sapphire. A bright line at the titanium - gold interface suggests that there may be an ultra-thin layer of an intermetallic compound created. It is questionable whether a layer of an alloy would increase or decrease the thermal conductivity. If it is a medium having properties between gold and titanium, it could decrease the dissimilarity and therefore improve the heat transfer. If the properties and the crystalline structure of the intermetallic compound are much different than the neighbouring 


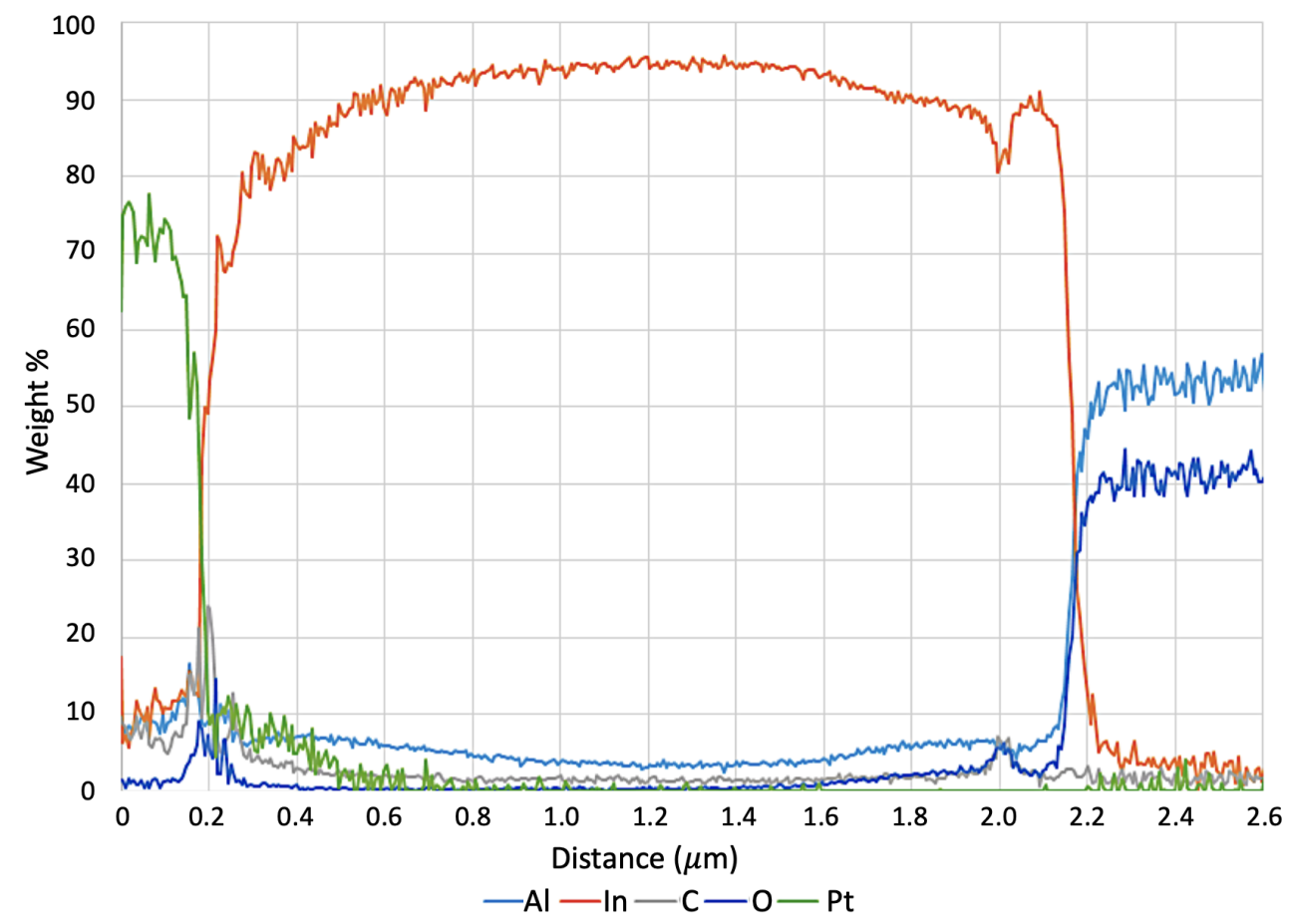

Figure 3.4: The line scan of atomic weight percent as a function of a distance across the thin film. The thickness of the indium film is $2 \mu \mathrm{m}$, and the trace quantities of other elements in the indium layer are insignificant. Before scanning the sapphire - indium sandwich was covered with a layer of platinum, visible below $0.2 \mu \mathrm{m}$. Above $2.2 \mu \mathrm{m}$ the only constituents are aluminium and oxygen in sapphire $-\mathrm{Al}_{2} \mathrm{O}_{3}$.

materials, it could create another obstacle for the heat transfer. A layer of platinum was deposited on the sapphire - $\mathrm{Ti}$ - Au sandwich before imaging, and it is visible in the atomic weight line scan below $0.35 \mathrm{~nm}$ (figure 3.6). The thickness of titanium equals to $(45 \pm 2) \mathrm{nm}$. The thickness of gold equals to $(520 \pm 22) \mathrm{nm}$. 


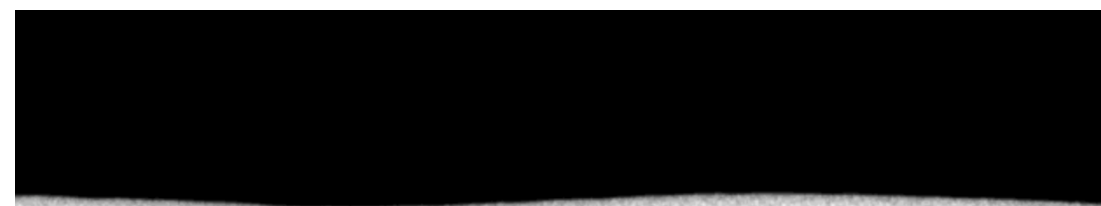

Pt

\section{$\mathrm{Au}$}

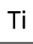

Sapphire

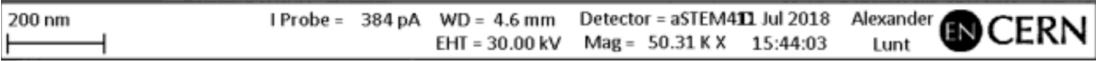

Figure 3.5: STEM image of the sapphire - Ti - Au sandwich. Both titanium and gold show a grainy, polycrystalline structure. A sharp interface between sapphire and titanium is visible. The bright line at the titanium - gold interface suggests that there may be an ultra-thin intermetallic layer created. A wavy upper surface of the gold layer is visible.

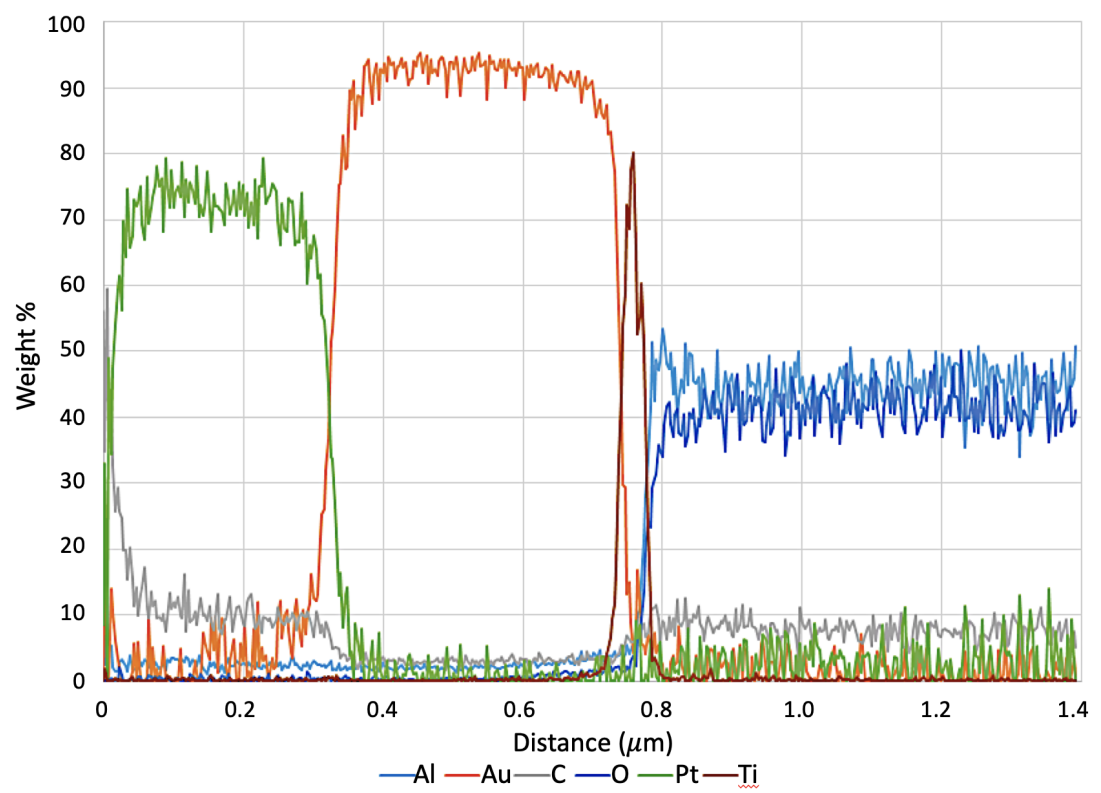

Figure 3.6: The line scan of atomic weight percentage as a function of the distance across the thin film. The thickness of Ti thin layer is approx $45 \mathrm{~nm}$ and of gold approx. $520 \mathrm{~nm}$. 


\subsection{Ultra-low temperature measurements in the Dilution Re- frigerator}

\subsubsection{The principle of achieving ultra-low temperatures}

The thermal performance of the sandwich setup at ultra-low temperatures was measured in the Cryolab DR, that provides continuous cooling power below $300 \mathrm{mK}$ [16]. A classic DR, so called "wet dilution refrigerator" is immersed in a bath of liquid helium, but there exist also "dry dilution refrigerators" pre-cooled by closed-cycle mechanical cryocoolers [69].

The cooling power of a dilution refrigerator comes from the heat of mixing of ${ }^{4} \mathrm{He}$ and ${ }^{3} \mathrm{He}$, because the enthalpy of diluted ${ }^{3} \mathrm{He}$ is larger than pure ${ }^{3} \mathrm{He} .{ }^{3} \mathrm{He}$, a lighter isotope of helium, is rare and therefore very expensive. It is created in a $\beta$ decay of tritium. One liter of gas at STP costs around $\$ 2000$ [70, and for its operation a dilution refrigerator needs a few tens of liters. A mixture of these isotopes of helium has a very particular property of separating in two phases below $0.87 \mathrm{~K}$ (figure 3.7). The concentrated phase consists almost only of pure ${ }^{3} \mathrm{He}$. The diluted phase contains mainly ${ }^{4} \mathrm{He}$ with certain percentage of ${ }^{3} \mathrm{He}$ diluted inside. The lower the temperature, the less ${ }^{3} \mathrm{He}$ in the diluted phase, but as one can see in figure 3.7, even close to absolute zero temperature there is always at least around $6.6 \%$ of ${ }^{3} \mathrm{He}$ in ${ }^{4} \mathrm{He}[71$. This very important property enables a continuous operation of a dilution refrigerator. ${ }^{4} \mathrm{He}$ stays liquefied in the internal part of the refrigerator, while ${ }^{3} \mathrm{He}$ is circulated through it. In the "wet" version of the device, after ${ }^{3} \mathrm{He}$ crosses the external pumps, it is purified in a $\mathrm{LN}_{2}$ trap, and precooled in the LHe bath. Then the tube enters the Inner Vacuum Chamber (IVC). In the IVC several internal shields reduce the radiation heat load. They are thermalised to the respective lower temperatures: at $4.2 \mathrm{~K}$ to the bath, at approx. $1.3 \mathrm{~K}$ to the $1 \mathrm{~K}$ pot, at approx. $0.7 \mathrm{~K}$ to the still, and at approx. $0.3 \mathrm{~K}$ to the heat exchanger. In the $1 \mathrm{~K}$ pot ${ }^{4} \mathrm{He}$ taken from the external bath is expanded in a Joule-Thomson valve, what reduces the temperature of the pot, flange and shield to around $1.3 \mathrm{~K}$. At the level of the still, the evaporation of ${ }^{3} \mathrm{He}$ cools it down to $0.7 \mathrm{~K}$. The incoming ${ }^{3} \mathrm{He}$ precooled in the bath and in the $1 \mathrm{~K}$ pot encounters a flow restriction - a needle valve, that regulates its condensation pressure. Then in a spiral tube it passes through the still and another small capillary constraining its flow, to ensure helium does not evaporate again. After a series of counterflow sintered heat exchangers it enters the heart of the device, where it mixes with ${ }^{4} \mathrm{He}$. The mixing chamber (MC) is the coldest place in the refrigerator with the temperature as low as a few milliKelvin. Diluted ${ }^{3} \mathrm{He}$ diffuses up to the still, where constant pumping reduces the pressure and evaporates the helium, mostly ${ }^{3} \mathrm{He}$. A higher partial pressure of ${ }^{3} \mathrm{He}$ than ${ }^{4} \mathrm{He}$ at a given temperature ensures that there is always significantly more ${ }^{3} \mathrm{He}$ evaporating than ${ }^{4} \mathrm{He}$. Thanks to the fact that in a ${ }^{3} \mathrm{He} /{ }^{4} \mathrm{He}$ mixture there is always at least $6.6 \%$ of ${ }^{3} \mathrm{He}$, it keeps diffusing from the mixing chamber 
to the still and constantly mixing with ${ }^{4} \mathrm{He}$, provides continuous cooling power. A scheme of the device with all the essential elements marked is presented in figure 3.7 .
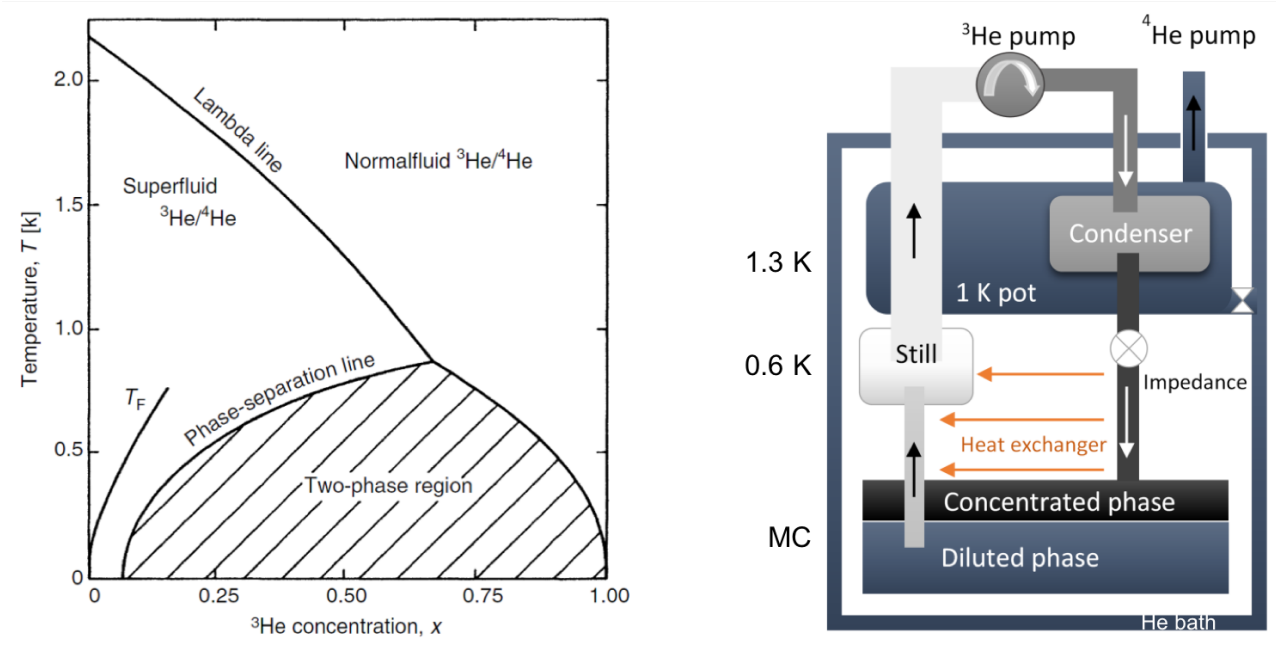

Figure 3.7: Left: phase separation of ${ }^{3} \mathrm{He} /{ }^{4} \mathrm{He}$ mixture 31] right: a scheme of a dilution refrigerator 72. MC stands for the mixing chamber.

\subsubsection{CERN Cryolab Dilution Refrigerator}

The Cryolab DR is an example of a classic type of the device, immersed in a bath of liquid helium. It was built in 2005 - 2006 following an in-house design by Patrick Wikus [73]. It is quite a big apparatus compared to some DRs available from industry, with an Inner Vacuum Chamber diameter of $310 \mathrm{~mm}$. In the IVC there are 3 radiation shields: at $1.3 \mathrm{~K}$ cooled by the $1 \mathrm{~K}$ pot, at $600 \mathrm{mK}$ $700 \mathrm{mK}$ cooled by the still, and the last one at around $200 \mathrm{mK}$. During a standard operation it consumes 100 liters of liquid helium per day, and it has a cooling power of $100 \mu \mathrm{W}$ at $60 \mathrm{mK}$. There are 50 liters STP gaseous ${ }^{3} \mathrm{He}$ circulating in a closed cycle. The mixing chamber of $112 \mathrm{~mm}$ diameter and $60 \mathrm{~mm}$ height, has a significant volume, which gives a possibility of conducting measurements of small samples at very low temperatures in the helium mixture itself. To increase the heat transfer between the helium mixture and the lid on which the samples are usually placed, a sintered copper heat exchanger was attached to the lid inside the MC with a layer of indium in between, what significantly increases the heat transfer surface to the mixture.

Preparations for a run of the DR take usually a few weeks and include: mounting of the sample, soldering the superconducting wires, closing the IVC, pumping and leak-testing the insert (frequently, repeated several times), inserting in the cryostat, pumping and leak-testing the outer part. The cool-down 
itself lasts about two days. First liquid helium is slowly transferred to the cryostat cooling it from room temperature to $4.2 \mathrm{~K}$. Then pumping on the $1 \mathrm{~K}$ pot reduces the temperature of the respective stage to $1.3 \mathrm{~K}$. Finally circulating and condensing the mixture of ${ }^{3} \mathrm{He} /{ }^{4} \mathrm{He}$ cools down the mixing chamber to around $20 \mathrm{mK}$. After a few weeks since the beginning of the cooldown, when all the residual heat loads diminished, by stopping the incoming flow and pumping on the still, it is possible to reach a temperature below $10 \mathrm{mK}$ 73. Such a state lasts only about half a day, until the moment when all the ${ }^{3} \mathrm{He}$ is evaporated. A standard measurement run of the DR lasts around 2 months, during which the machine requires frequent attention, so the noble goal of reaching temperatures close to the absolute zero, has to be paid for with a lot of patience and persistence form the side of the operator. The view on top of the cryostat with all the connections to pumps, pressure sensors, amplifiers for temperature sensors, etc, is shown in figure 3.8. The insert of the Cryolab DR with all the elements marked is presented in figure 3.9 .

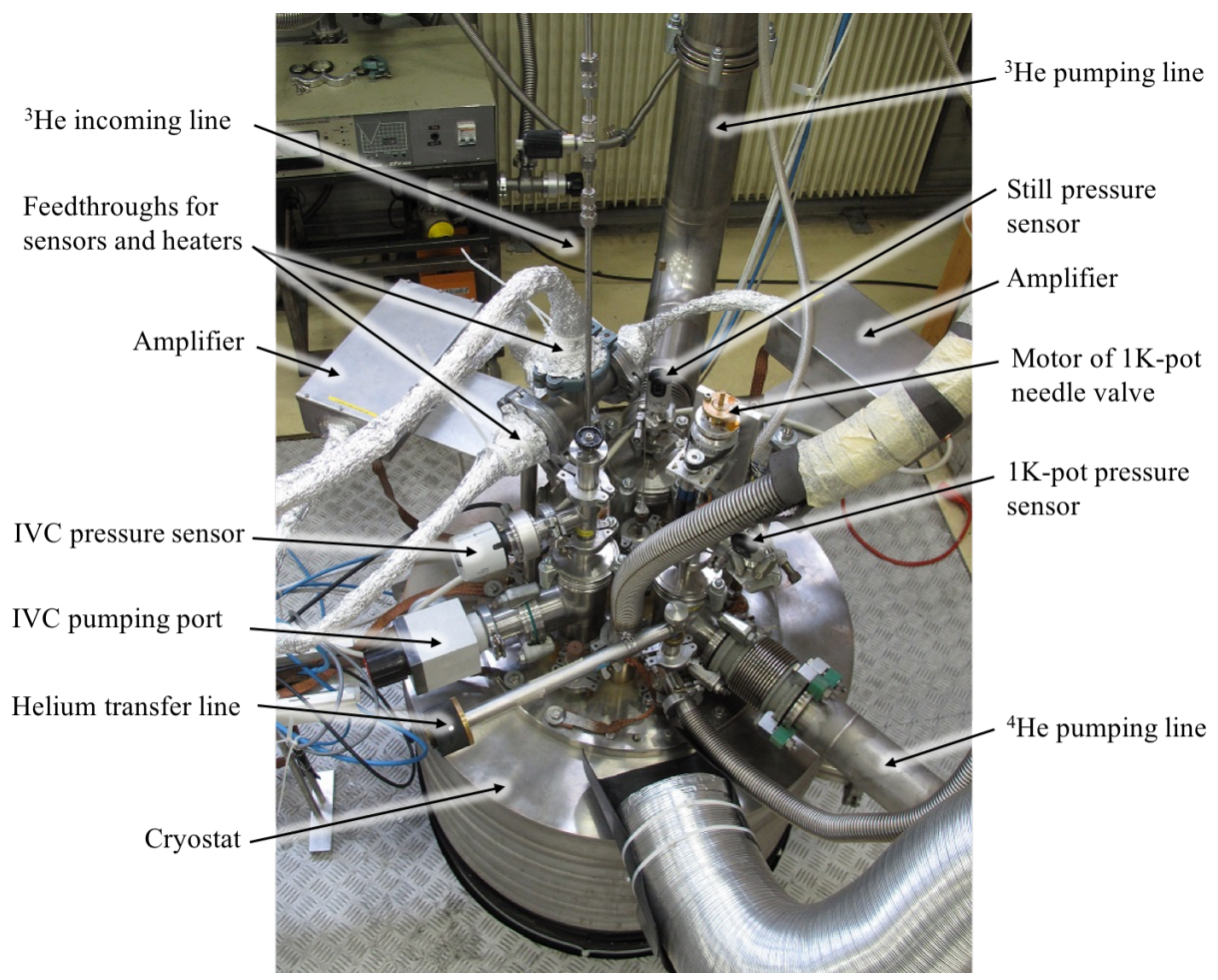

Figure 3.8: The top of DR's cryostat with all the connections marked. 


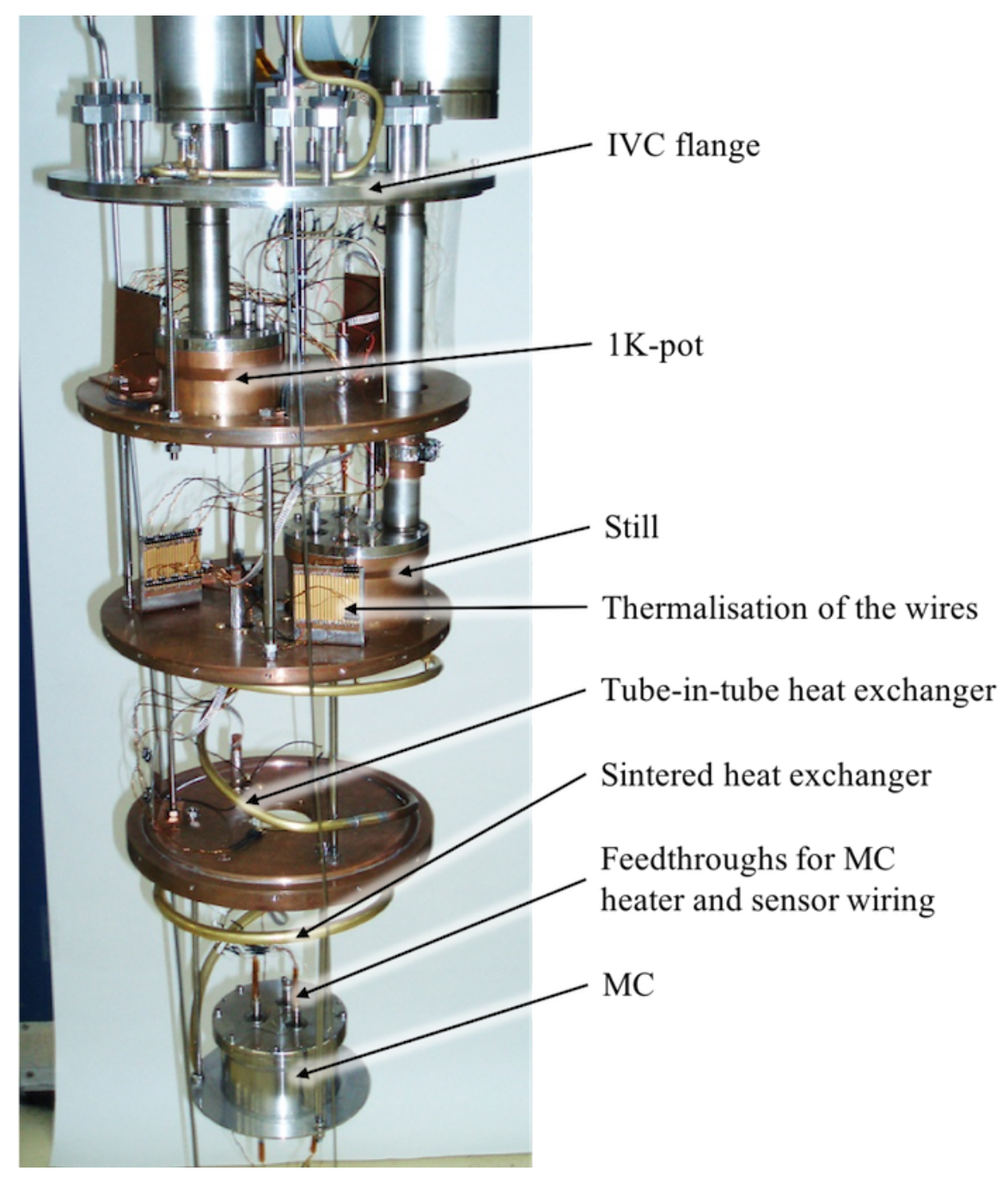

Figure 3.9: Insert of the CERN Cryolab Dilution Refrigerator as built in 2006 with the most important elements described.

\subsection{Mechanical crycoolers}

The main interest of the presented study focuses on temperatures in the milliKelvin temperature range, where the properties of materials and interfaces are not yet fully explained. However, measurements at low temperatures around a few Kelvin are significantly easier than running a dilution refrigerator. Finding a way to estimate the ultra-low temperature performance of the electrodes, by measuring their low temperature properties, would be a very valuable and relatively simple way of validating them. Above $1.5 \mathrm{~K}$ the measurements can be done in a standard helium bath cryostat, where by reducing the pressure above 
liquid helium $\left({ }^{4} \mathrm{He}\right)$ its temperature is also reduced. Another method to cool down well below the temperature of liquid nitrogen is with use of a cryocooler. Cryocoolers are compact, freely standing devices, not requiring any cryogens, what makes them extremely convenient to use. Therefore the sandwich setup was installed on one of the Cryolab cryocoolers, where its low temperature properties were characterised. The measurements were performed as in the DR: by heating on the stamp and measuring the temperature difference between the stamp and the platform. The sapphire - Ti - Au - In - Cu sandwich setup installed on the cold head of the cryocooler with sensors and a heater attached is presented in figure 3.10 .

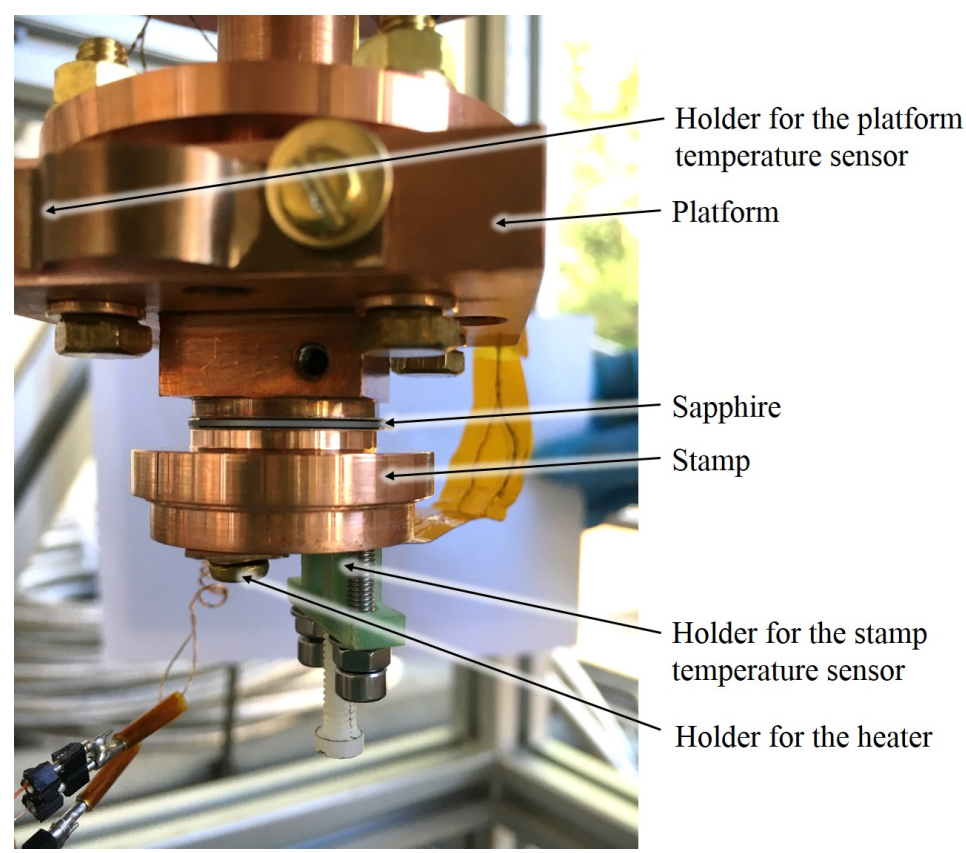

Figure 3.10: The sapphire - $\mathrm{Ti}$ - $\mathrm{Au}$ - In - Cu setup mounted on a cryocooler for a low temperature characterisation.

There are several types of mechanical cryocoolers, characterized with the presence of a high heat capacity regenerator material. In a standard thermal cycle, the gas is compressed at the warm end of the device, the heat of compression is removed, then it flows to the cold end where it expands, cools down and absorbs heat from its surroundings. A phase shift between the cycles of pressure and volume change gives the actual cooling effect. Even assuming the gas is ideal and the heat exchangers are perfect, because of the laws of thermodynamics limiting the adiabatic processes, the low temperature point of a such cycle is still quite far from what we consider to be a "low temperature" in cryogenics. The presence of a high heat capacity regenerator is essential to obtain heat storage during the cycle and allows achieving much lower tempera- 
tures. The three basic types of cryocoolers are: Stirling, Gifford-McMahon and a pulse tube. A brief introduction to the different types will present the cooling capabilities, the related pros and cons of their operation and background influences to thermal measurements.

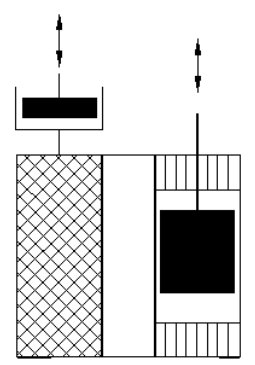

a) Stirling-cryocooler

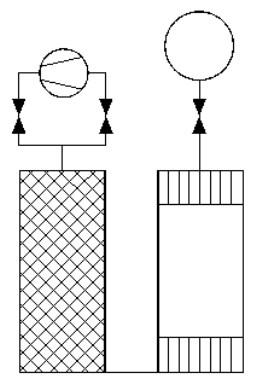

c) Orifice pulse tube (PT)

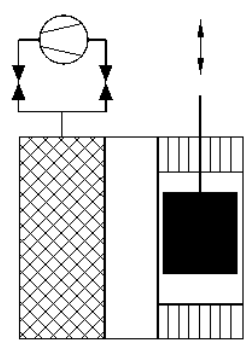

b) Gifford-McMahon

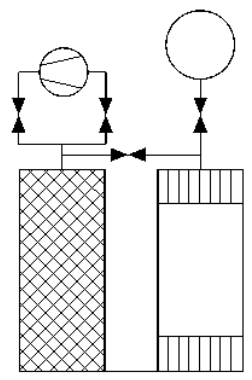

d) Double inlet PT

Figure 3.11: Schemes of different types of mechanical coolers: a) Stirling type, b) GiffordMacMahon, c) Pulse tube with a buffer and the orifice, d) Double inlet PT. In reality the displacer and the regenerator are frequently integrated, i.e. the displacer is made of the regenerator material itself. Schemes by Torsten Köttig.

\subsubsection{Stirling and Gifford-MacMahon cycles}

The Stirling thermodynamic cycle requires the displacer (responsible for displacing the gas from the warm end to the cold end of the cooler) and the piston (responsible for the compression of the gas) to move with $90^{\circ}$ phase shift. Moreover, the moving parts introduce mechanical vibrations to the system. Pressure, volume, cooling power and all other parameters typically change with a frequency of 50-60 Hz. A scheme of a Stirling type cryocooler is presented in figure $3.11 \mathrm{a}$.

In a Grifford-MacMahon (GM) type of a cryocooler, the moving piston is replaced by a compressor and a set of valves or a singular rotary valve, which provides the pressure change in a proper phase shift with respect to the position of the internal displacer. The compression - expansion cycle is the same as in 
a case of a Stirling cryocooler. There is no piston, but the moving displacer still introduces certain mechanical vibrations to the system. A scheme of a GM cryocooler is presented in figure $3.11 \mathrm{~b}$. The displacer typically contains the regenerator material, making it an oscillating mass of around $1 \mathrm{~kg}$, which causes the main source of mechanical vibrations in both the Stirling and the GM type of a cryocooler. A very characteristic phenomenon present in the GM cryocoolers is a peak of mechanical vibration and temperature oscillation around the operating frequency, usually around 1-2 Hz, which may disturb low frequency dynamic measurements.

\subsubsection{Pulse Tube Refrigerator (PTR)}

A basic pulse tube consists of a compressor with a rotary valve, a fixed regenerator and a tube filled with gas, but without the displacer. Only the low temperature side of the pulse tube itself is connected with the regenerator. Most of the gas particles stay in the pulse tube itself, cyclically changing their positions along the tube, as well as pressure and temperature, creating a virtual gas piston. It is assumed that there is no turbulence or convection of the gas in the pulse tube. The open tube makes the device sensitive to the orientation - it works best in vertical position with the cold end facing downwards (with respect to gravity). Because of the lack of a mechanical displacer, a small phase shift between the pressure and volume exists only thanks to the presence of a heat exchanger at the warm end of the pulse tube. It is definitely not enough to achieve any useful cooling power. An implementation of an additional element introducing a phase shift is necessary for the efficiency of the device. Therefore, in most of the PTRs there is an orifice that leads to a buffer volume at the warm end of the tube. The buffer volume is a few times bigger than the volume of the gas inside the device. The orifice and buffer act as a resistance and capacity and create the necessary phase shift. A scheme of a PTR with an orifice and a buffer volume is presented in figure 3.11 c. The absence of moving elements in the cold part makes the device almost vibration free compared to the Stirling or GM cooler. A pulse tube refrigerator can be further improved by applying a second orifice connecting the compressor with the warm end of the pulse tube (fig. $3.11 \mathrm{~d}$ ). That type of a refrigerator is called a double inlet pulse tube. In this configuration part of the gas entering the buffer responsible for the phase shift, comes directly from the high pressure side of the compressor without passing through the regenerator and the pulse tube. In order to reach lower temperatures, the cryocoolers consist of two or even three stages. The typical cooling power of a double stage PTR is around $1 \mathrm{~W}$ at $4.2 \mathrm{~K}$ and $40 \mathrm{~W}$ at $45 \mathrm{~K}$. GM cryocoolers and double inlet PTRs are easily available industrially and commonly used. A much more profound description of the various types of cryocoolers can be found in multiple sources, e.g. the "Art of Cryogenics" by G. Ventura 71 . 


\subsubsection{Cryocoolers used in the CERN Cryolab}

There are several mechanical coolers used in the Cryolab for various types of measurements. Because of the simplicy of operation in a wide temperature range, and an absence of mechanical vibrations, the low temperature measurements of the sandwich sample were performed on a two-stage pulse tube refrigerator. The investigated temperature range was between $3 \mathrm{~K}$ and $60 \mathrm{~K}$.

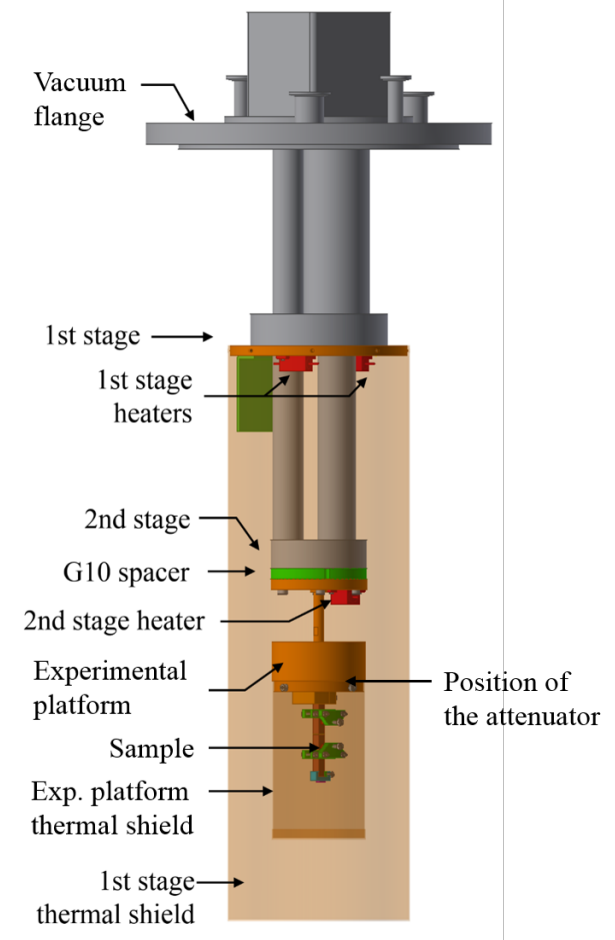

Figure 3.12: A scheme of the two-stage PTR used for measuring the sandwich 74

Steady state and dynamic measurements with a heat input in a form of a sine wave were performed, like in the DR. As mentioned before, the disadvantage of using a cryocooler for dynamic measurements is that an interefrence appears for frequencies of the thermal excitation close to the operating frequency of the cryocooler. This resonance makes an accurate measurement impossible. The Cryolab team solved the problem by installing a special thermal attenuator consisting of several interwoven layers of copper, lead and stainless steel following [75]. Thanks to its multiple interfaces, and a smart combination of materials with high heat capacity and high and low conductivities, it attenuates the amplitude of temperature wave from several tens or even hundreds of milliKelvin to well below $1 \mathrm{mK}$. The improvement of the signal thanks to the installation of the attenuator the reader can find in [72]. A scheme of the PTR used for the measurements of the sandwich setup is presented in figure 
3.12 , whereas a picture of the attenuator manufactured by the Cryolab team is shown in figure 3.13 .

A detailed description of the instrumentation used during the measurement campaigns is presented in chapter 4 and further information about measurements performed in the DR and on the pulse tube cryocooler are presented in chapter 6 .
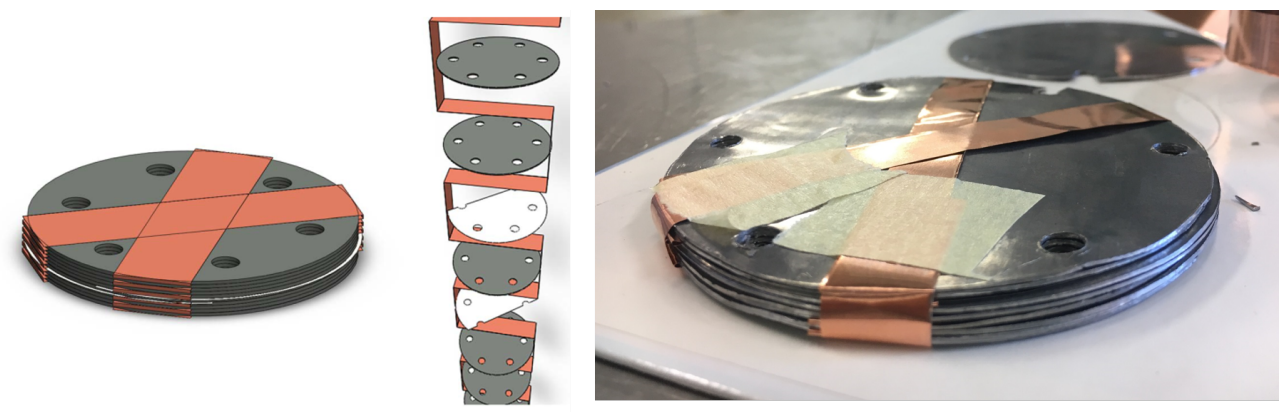

Figure 3.13: Left: Drawing of the low temperature attenuator of thermal oscillations copied from [75. Right: photo of the thermal attenuator for the pulse tube cryocooler manufactured according to 75 by the Cryolab team. Multiple disks made of lead and stainless steel with a copper ribbon interwoven between them are visible. 


\section{Chapter 4}

\section{Measurement methodology, instrumentation and accuracy}

The precision of any type of measurement can be a significant issue. The problem becomes even bigger, when dealing with temperatures close to absolute zero. Besides using a suitable equipment, one has to pay special attention to the measurement method, trying to minimize all the possible unwanted influences that could deteriorate measured signals.

\subsection{Steady state measurement method}

In steady state measurements, the temperature of the mixing chamber was kept constant by using a Picowatt TS-530A temperature controller [76]. The heat load was applied to the stamp with an electric heater while the temperatures of the stamp and the platform were measured (both elements marked in figure 3.1). A plot of both temperatures as a function of the applied heat load is presented in figure 4.1. Knowing the temperature difference, the heat load and the cross-section, one can calculate the thermal resistivity $R=A \cdot \Delta T / \dot{Q}$. However, because of its strong temperature dependence, described in section 2.4 the plot of resistivity as a function of temperature presented in figure 4.2 is not very informative. Since the temperature dependence of a perfect Kapitza-like interface resistance is $R=\kappa T^{-3}$ (following eq. (2.21)), plotting the multiplication of resistivity and temperature to the power of three: $R T^{3}=$ $\kappa$, one should get a constant. Such a way of representing the data, based on the small temperature gradients assumption, is frequently found in literature. 


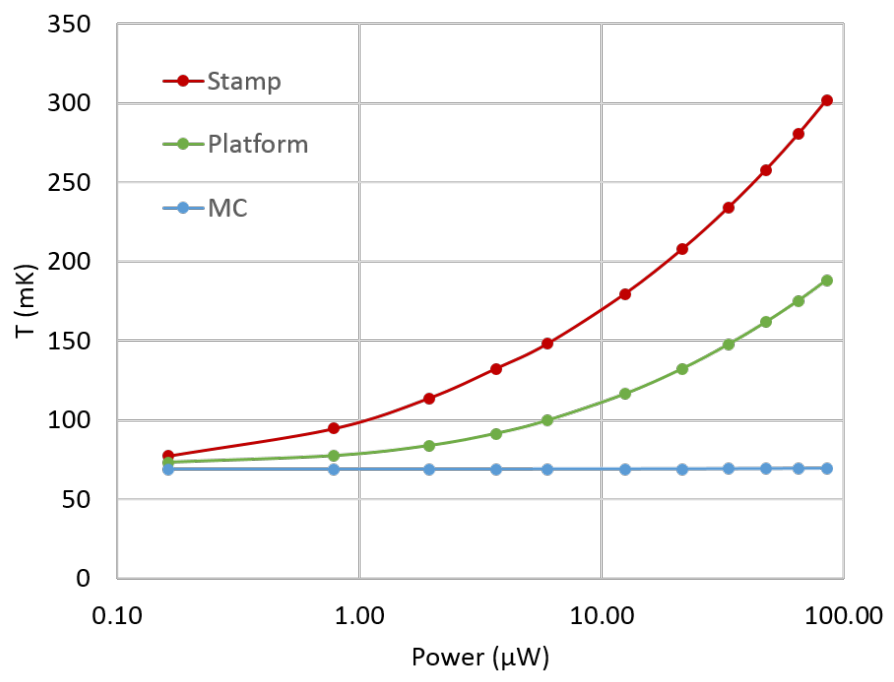

Figure 4.1: Temperatures of the stamp, platform and the mixing chamber as a function of heat load applied to the stamp. Example for the compressed setup, without magnetic field, mixing chamber temperature kept constant at approx. $70 \mathrm{mK}$.

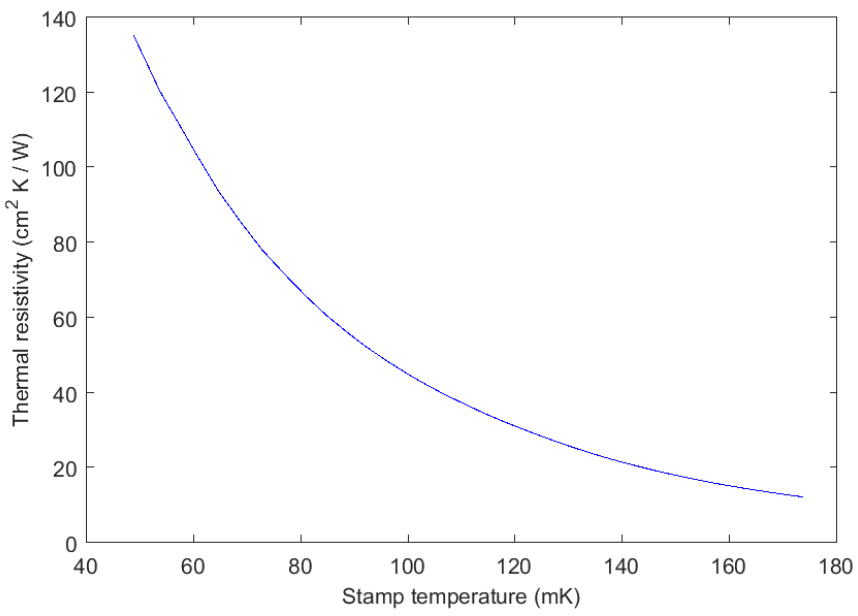

Figure 4.2: Thermal resistivity calculated as $R=A \cdot \Delta T / \dot{Q}$ of the compressed sandwich setup with indium in normal conducting state as a function of the stamp temperature, for the mixing chamber temperature of $20 \mathrm{mK}$. A strong temperature dependence is visible, what justifies the use of eq. 4.1.

The gradient of temperature during the measurements in the Cryolab DR is frequently higher than the absolute temperature, eg. $\Delta T=50 \mathrm{mK}$ at $T_{\mathrm{pl}}=$ $30 \mathrm{mK}$. For such a case, the thermal interface resistivity $\kappa_{\text {interface }}$ in the unit of $\mathrm{cm}^{2} \mathrm{~K}^{4} / \mathrm{W}$ can be calculated according to 77,78$]$ as: 


$$
\kappa_{\text {interface }}=\frac{A\left(T_{\mathrm{h}}^{4}-T_{\mathrm{c}}^{4}\right)}{4 \dot{Q}},
$$

where $T_{h}$ and $T_{c}$ are the temperatures on the hot side and the cold side of the interface, $\dot{Q}$ is the heat load applied to the hot end (on the stamp), and $A=3.14 \mathrm{~cm}^{2}$ is the area of the interface. Using equation 4.1 for relatively large temperature gradients, one cannot expect the result to be constant and a certain linear temperature dependence is visible. A plot of the thermal resistivity of the same setup configuration as presented in figure 4.2 (compressed sandwich with indium normal conducting, mixing chamber temperature $20 \mathrm{mK}$ ), but calculated with eq. 4.1) is presented in figure 4.3 .

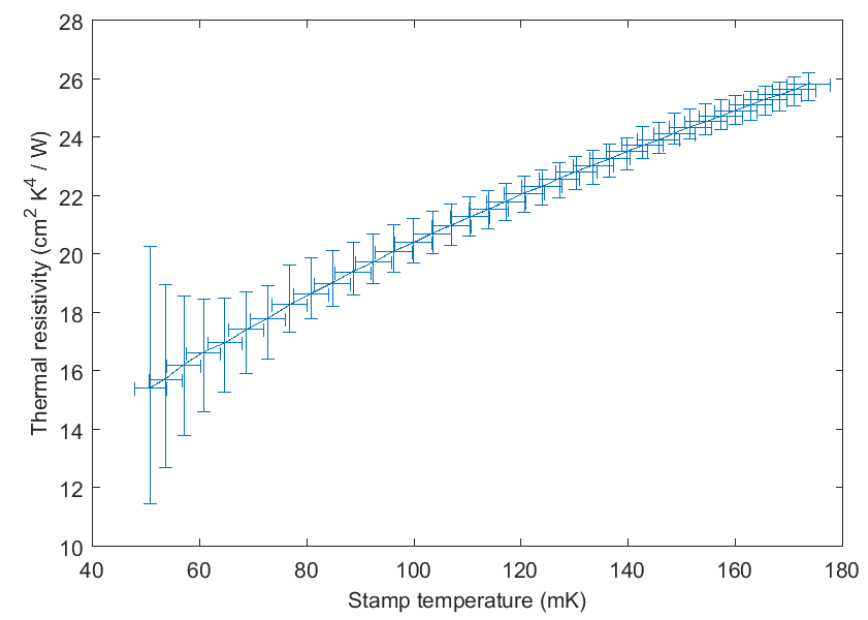

Figure 4.3: Thermal resistivity of the compressed sandwich with indium in normal conducting state, calculated acc. to eq. 4.1 as a function of the stamp temperature. The temperature of the mixing chamber was kept constant at $20 \mathrm{mK}$. A plot in a form of a horizontal line would indicate a perfect Kapitza-like behaviour for the small gradients assumption.

The interface thermal resistivity calculated with equation (4.1) requires a precise measurement of the two temperatures, the power dissipated in the heater, and the cross-sectional area. There are several factors influencing the precision of the measurement in the Cryolab DR:

- the accuracy of the sensor calibration done in-house and the uncertainty of the reference devices, in particular LakeShore U02611 RuO sensor and the Fixed Point Device SRD1000;

- the accuracy of equipment in the measurement chain:

- Picowatt resistance bridges AVS-47A and AVS-47B for the temperature measurement, 
- multimeters Keithley 2000 reading the voltage and current for the power determination;

- the surface A depends on the manufacturing perfection and in all the measured configurations it is determined by the diameter of the stamp $\varnothing 20 \pm 0.1 \mathrm{~mm}$, which results in a cross-sectional area of $3.14 \pm 0.03 \mathrm{~cm}^{2}$;

- the choice of the temperature sensor excitation, to make a trade off between sensor self-heating and voltage signal level;

- filtering, scrupulousness of grounding and the noise picked up by the wiring in and outside of the cryostat.

This chapter presents the method of uncertainty estimation, that includes all of the above mentioned factors for the measurement of the temperature and power.

\subsubsection{Sensors used for the ultra-low temperature measurements}

There were three types of sensors used in the measurement campaigns. The first type is a LakeShore Ruthenium Oxide $(\mathrm{RuO})$ sensor in an AA package [79] originally calibrated by the manufacturer and fitted with a Chebyshev curve 80,81. Ruthenium oxide has a very characteristic property of a negative $\mathrm{dR} / \mathrm{dT}$ dependence and a high sensitivity at very low temperatures. Sensor number U01826 is always in the MC, and usually referred to as the "Mixing Chamber sensor". Originally calibrated in a range from $50 \mathrm{mK}$ to $40 \mathrm{~K} 81$ and extrapolated down to $20 \mathrm{mK}$ by previous researchers working with the Cryolab DR [4, 82. It gives an indication of the base temperature for each measurement. Sensor number U02611, originally calibrated down to $50 \mathrm{mK}$ by LakeShore 80 has been permanently glued with Stycast ${ }^{\circledR}$ into a little copper block, which allows to mount it with an M3 screw to any other surface with a corresponding thread. Its calibration was extended towards low temperatures with the use of a Fixed Point SRD1000 83 85] device. It served together with the Fixed Point Device, described in section 4.1.2, as a reference to calibrate most of the other sensors, therefore it is usually referred to as the "reference LS sensor".

The second type is also a LakeShore $\mathrm{RuO}$ sensor in an AA package, but delivered without a calibration curve. Four sensors of that type were calibrated in one of the DR runs with respect to the Fixed Point SRD1000 device [8385. These sensors were mounted according to the instruction given by the manufacturer, i.e. placed in a copper block in a cylindrical hole of a diameter $0.1 \mathrm{~mm}$ bigger than the diameter of the AA package, with a thin layer of Apiezon $\mathrm{N}^{\circledR}$ grease 86 in between. Despite high sensitivity, which is certainly their advantage, these sensors cannot be applied in some places of the setup, because of relatively big dimensions. Out of the 4 calibrated sensors (certain 
redundancy is necessary in case of unexpected malfunction), only two were actually used in measurements. These two sensors, numbered Rx61 and Rx66, were always placed on the platform, and therefore usually referred to just as the "platform sensor".

The third type is a small bare chip with a layer of $\mathrm{RuO}$ thick film, of around $0.9 \mathrm{kOhm}$ resistance at room temperature. They were glued with Stycast ${ }^{\circledR}$ to very small $(4 \times 6 \mathrm{~mm})$ copper plates with a LakeShore Quad-Lead ${ }^{\mathrm{TM}}$ phosphor bronze wire [79] soldered to it. Five sensors of that type, designated Rx0, Rx1, $\mathrm{Rx} 2$, Rx3, Rx4, were prepared, thermally cycled, and calibrated (see section 4.2. . Their small dimensions make it easy to mount in almost any place of the setup. Their maximum resistance at low temperature does not exceed 20 $\mathrm{kOhm}$, what makes them a bit less sensitive, compared to Rx61 and Rx66. Sensor Rx4 was all the time placed at the stamp and is referred to as the "stamp sensor". In some measurement runs sensor Rx2 was placed on the lid of the Mixing Chamber just below the platform, and therefore is referred to as the "lid sensor". A comparison of the sensitivities of bare chips, represented by sensor Rx4, and AA packages, represented by sensor Rx66 is shown in figure 4.4. The sensitivity of the AA packages is at least an order of magnitude higher than the bare chips.

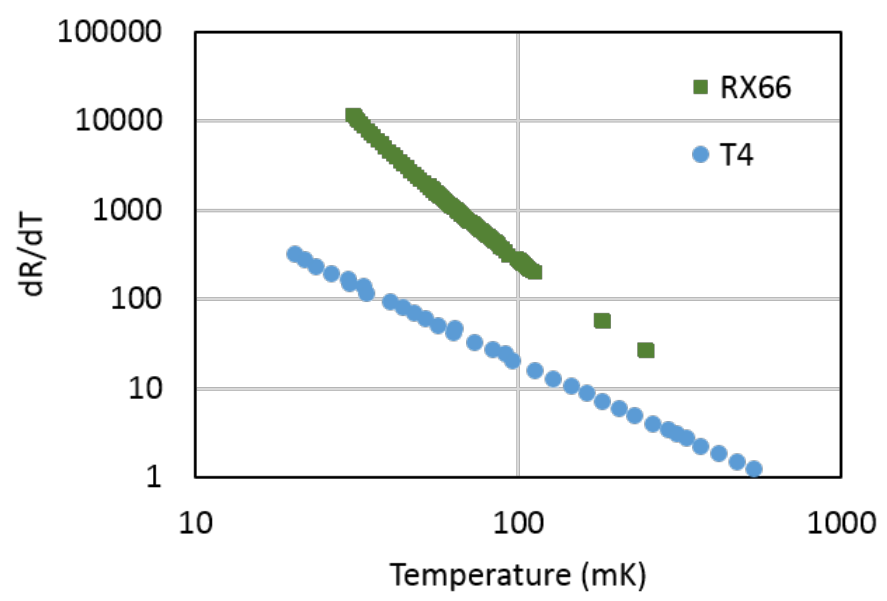

Figure 4.4: Sensitivity of sensor RX66 (AA package) and Rx4 (bare RuO chip) as a function of temperature. The sensitivity of AA packages is at least one order of magnitude higher than the bare $\mathrm{RuO}$ chips.

\subsubsection{Superconducting Fixed Point Device SRD1000}

None of the original calibration curves of the sensors used around the MC goes below $50 \mathrm{mK}$. A reasonable extrapolation down to $30 \mathrm{mK}$ was made, based on a typical experimental behaviour, but its accuracy should be verified to 
make sure the measurements match the required precision. The superconducting Fixed Point Device SRD1000 provided by Hightech Development Leiden 84] was used to confirm the accuracy of the ultra-low temperature points. The working principle of the SRD1000 is based on transition points of several superconducting materials with very precisely defined properties. The device mounted in the Cryolab DR has six transition points at temperatures ranging from $15 \mathrm{mK}$ to $1.2 \mathrm{~K}$. For each point the following values are defined: $T_{\mathrm{SC}}$ - the temperature when the sample is fully superconducting; $T_{\mathrm{NC}}$ - the temperature when the sample is fully normal conducting; $T_{\mathrm{C}}$ - the transition temperature (when half of the transition is completed); $W_{\mathrm{C}}=T_{90}-T_{10}$ - width of the transition as marked in figure 4.5 , where $T_{90}$ and $T_{10}$ are the temperatures after completing $10 \%$ and $90 \%$ of the transition; $U_{C T \%}$ - relative uncertainty.

All the reference materials with their corresponding transition temperatures, as well as the width of the transition $W_{\mathrm{C}}$, and the relative uncertainty $\mathrm{U}_{\mathrm{CT} \%}$ are given in table 4.1. It is remarkable that the uncertainty of none of the transition temperatures exceeds $2 \%$.

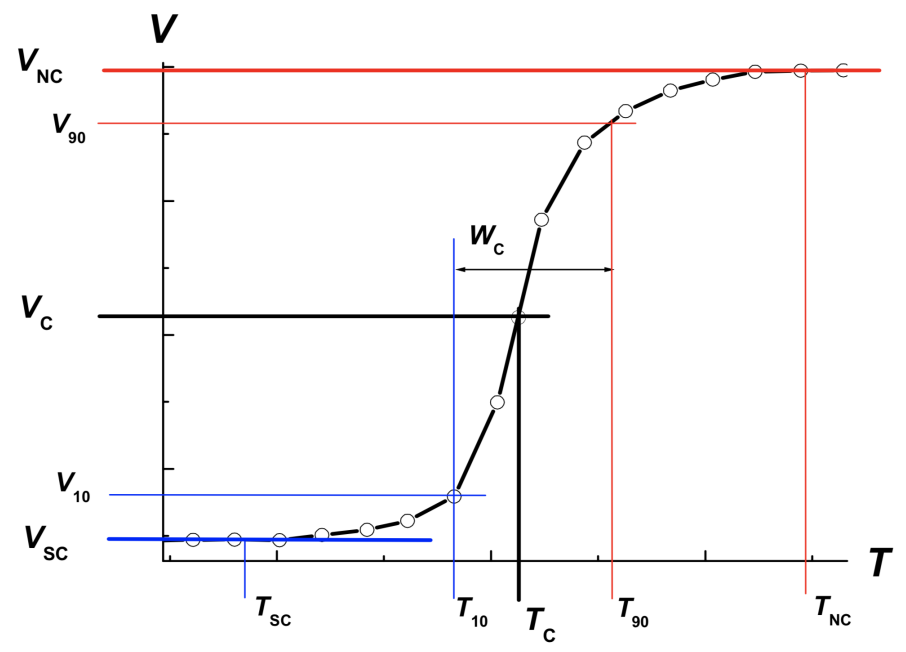

Figure 4.5: Voltage of the SRD1000 as a function of temperature during the transition of one of the points, copied from the manual 85

The superconducting samples are placed in an array of transformers with constant voltage supplied to the primary coils. Their transitions at given temperatures are detected by a change of voltage in the secondary coils. A scheme of the circuitry and sensor connections is presented in figure 4.6.

External disturbances are shielded by a $\mathrm{Nb}$ shield and a cryoperm shield. The cryoperm shield of the SRD1000 should be demagnetized before inserting in the DR with a DCS-20 degauss tool as indicated in the manual [84]. Any residual magnetic flux trapped in the $\mathrm{Nb}$ shield could shift the transition points and falsify the calibration result. 
Table 4.1: The temperatures $T_{\mathrm{SC}}, T_{\mathrm{NC}}$, the width of the transition $W_{C}$, and their relative uncertainty for the superconducting Fixed Point Device SRD1000 \#S301 85] installed in the Cryolab DR.

\begin{tabular}{|c|c|c|c|c|c|c|}
\hline$\#$ & Material & $T_{\mathrm{SC}}(\mathrm{mK})$ & $T_{\mathrm{NC}}(\mathrm{mK})$ & $T_{\mathrm{C}}(\mathrm{mK})$ & $W_{\mathrm{C}}(\mathrm{mK})$ & $U_{\mathrm{CT} \%}$ \\
\hline 1 & $\mathrm{~W}$ & 13.5 & 17.5 & 14.4 & 0.6 & $0.8-2$ \\
2 & $\mathrm{Be}$ & 19.5 & 21.5 & 20.7 & 0.4 & $0.4-2$ \\
3 & $\mathrm{Ir}_{80} \mathrm{Rh}_{20}$ & 31 & 36 & 35.0 & 1 & $0.6-2$ \\
4 & $\mathrm{Ir}_{92} \mathrm{Rh}_{8}$ & 62 & 66 & 64.3 & 0.7 & $0.2-2$ \\
5 & $\mathrm{Ir}$ & 92 & 98 & 93.7 & 1.5 & $0.3-2$ \\
6 & $\mathrm{Al}$ & 1180 & 1185 & 1183 & 1 & $0.02-2$ \\
\hline
\end{tabular}
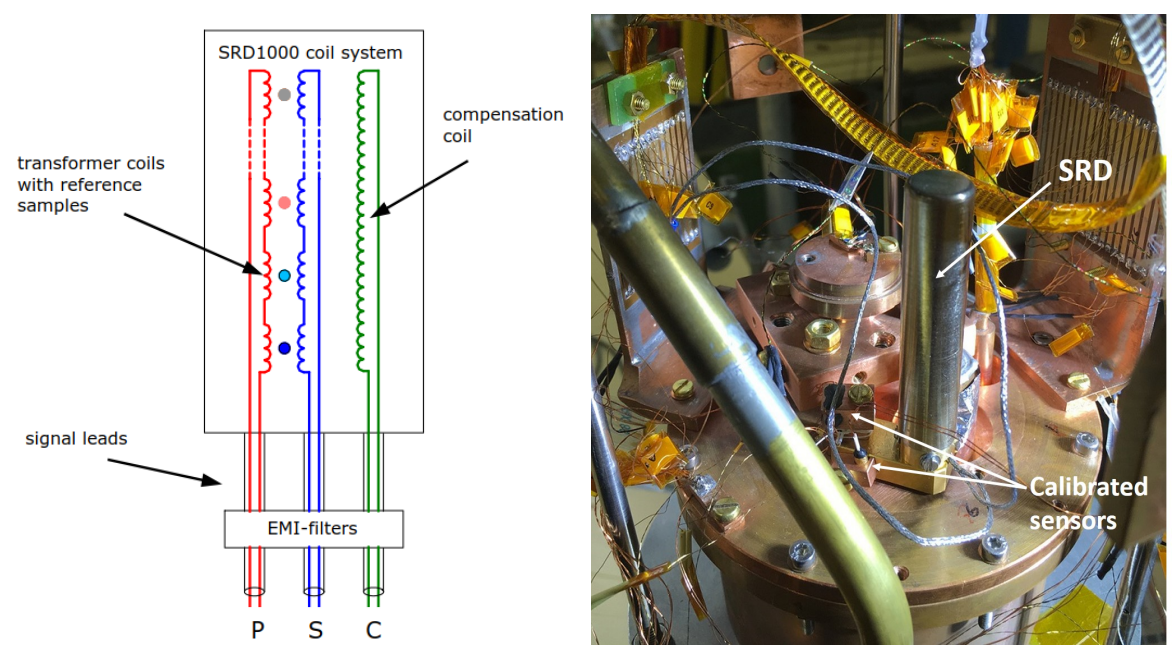

Figure 4.6: Left: A scheme of the SRD1000 transformer coils copied from its manual 84. Right: The SRD 1000 on the lid of the MC with sensors mounted on the golden base; the stamp and the platform visible just behind the SRD.

The SRD was carefully mounted on the lid of the mixing chamber with several sensors placed on its golden base for calibration. To ensure the temperature uniformity of the samples and its surroundings during calibration, the whole mixing chamber was varied in temperature with an internal MC heater. Once a transition point was found, it was kept half way between the fully normal and superconducting states for several minutes by precisely controlling the power applied to the MC heater. In that time the sensors were scanned through and their resistance recorded for obtaining a suitable $T(R)$ dependence. The SRD device mounted on the lid of the $\mathrm{MC}$ with sensors attached and ready for calibration is presented in figure 4.6 on the right. 


\subsection{Sensor calibration in the ultra-low temperature range}

\subsubsection{Thermal cycling of temperature sensors}

The LakeShore $\mathrm{RuO}$ sensors in AA packages are delivered in a ready-to-use condition. The RuO bare chips on the other hand should be thermally cycled before installation, to develop a sufficient amount of micro-cracks in the thick film of $\mathrm{RuO}$, that could ensure a stability of its properties $82,87,89$. Once cycled and calibrated, the sensors should not be heated up above room temperature, because it anneals the cracks and changes the temperature - resistance dependence. They should be kept in dry conditions, as they have a tendency to absorb humidity, what also changes their properties. All bare chips have been mounted on a copper flange, closed in a vacuum chamber and cycled 60 times between room and liquid nitrogen temperatures. Afterwards they were calibrated, and those showing best behaviour ( $\mathrm{Rx} 0, \mathrm{Rx} 2$ and $\mathrm{Rx} 4)$, i.e. following the theory [31,71] and our expectations, were used in measurement campaigns.

\subsubsection{Bare chips}

The small $\mathrm{RuO}$ bare chips were the first temperature sensors used in this study. After the thermal cycling, they were all mounted on a copper platform, tightly surrounded by a radiation shield and calibrated versus the reference LS sensor. They were calibrated in two ranges: the more important, lower temperature range $20 \mathrm{mK}$ to $300 \mathrm{mK}$, and the higher, less important, temperature range $300 \mathrm{mK}$ to $3.6 \mathrm{~K}$.

According to [31], a $\mathrm{RuO}$ thick film $\mathrm{R}(\mathrm{T})$ dependence below $1 \mathrm{~K}$ should follow an equation:

$$
R=R_{0} \exp \left[\left(T_{0} / T\right)^{-0.345}\right] .
$$

Then the resistance of a sensor plotted versus $T^{-0.345}$ should result in a straight line. Our sensors are apparently not exactly following that equation, and therefore the plot does not represent a straight line, see figure 4.7. Only the reference LS sensor (U02611) behaves as described by the theory.

G. Ventura in Art of Cryogenics [71 cites Mott's law stating that the R(T) dependence of some amorphous materials and oxides below $1 \mathrm{~K}$ follows:

$$
R=R_{0} \exp \left[\left(T_{0} / T\right)^{n}\right]
$$

where $n \in\langle 0.25 ; 0.5\rangle$ and $T_{0} \in\langle 1 K ; 100 K\rangle$. This equation was applied to the $0.9 \mathrm{kOhm}$ bare chips. Their resistance, plotted versus $T^{-n}$ and with logarithmic scale on the vertical axis is again almost a straight line, but not sufficiently precise, see figure 4.8. The exponents found from the fit are summarised in table 4.2 .

Since the behaviour of bare chip sensors does not follow the expected theory, I have decided to use a curve that would minimize the residuals of the fit similarly to equations 4.2 and 4.3 , but thanks to additional parameters, would 


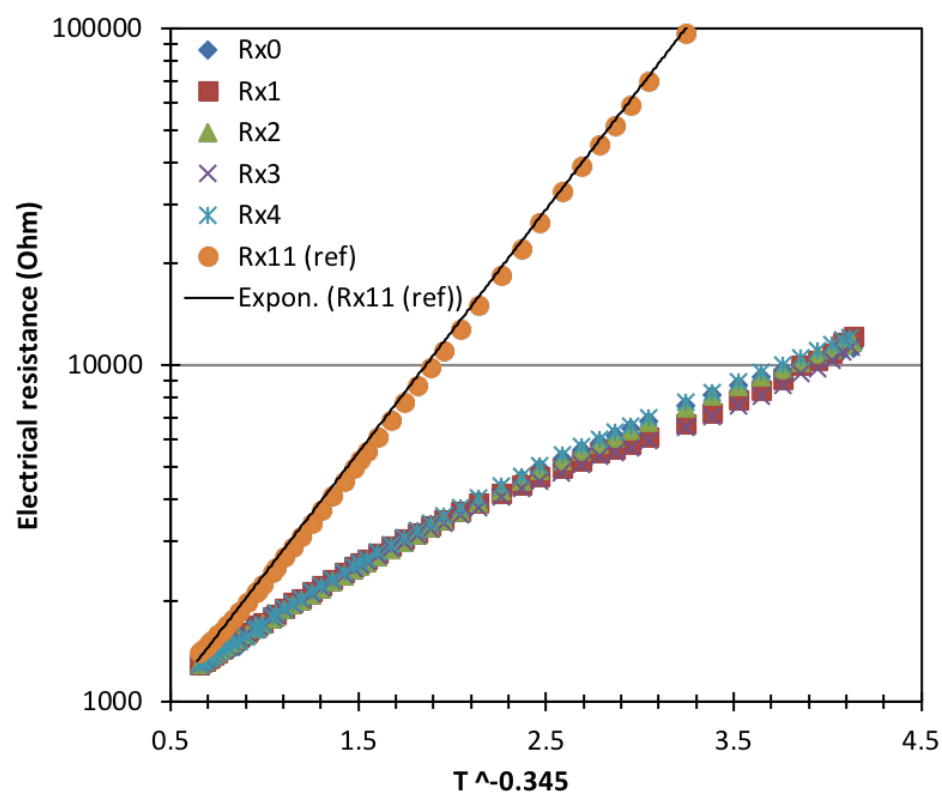

Figure 4.7: Calibration of the sensors with an equation $R=R_{0} \exp \left[\left(T_{0} / T\right)^{-0.345}\right]$. Only the original U02611 shows the behaviour predicted by the theory (eq. 4.2 .

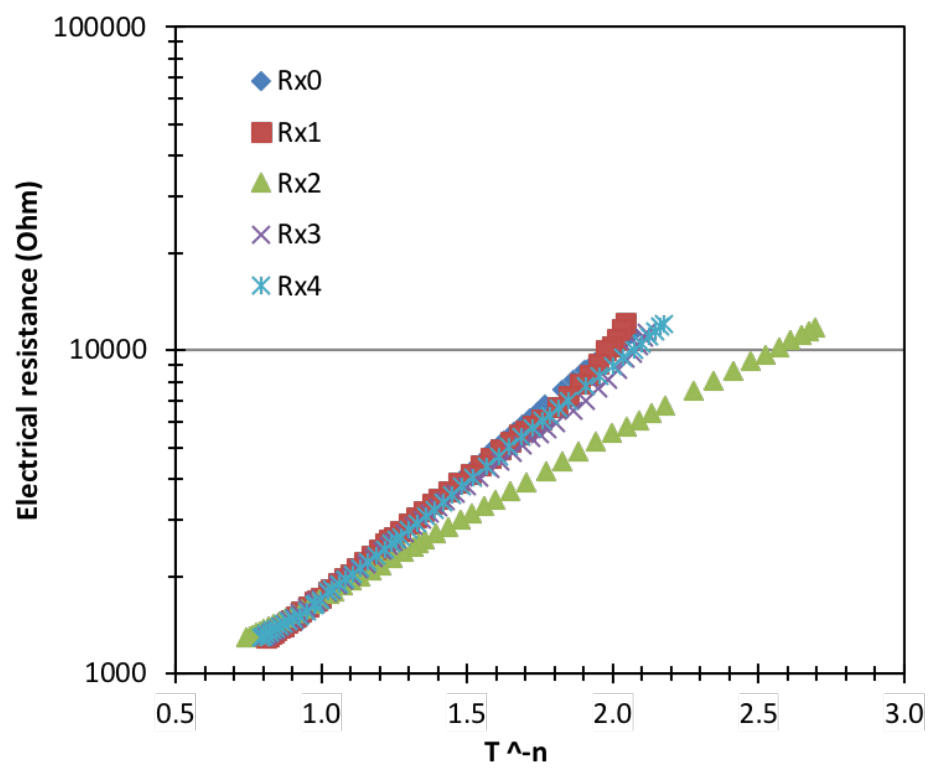

Figure 4.8: Calibration of the sensors according to Mott's law with equation 4.3. Some sensors demonstrate "better" behaviour ( $\mathrm{Rx} 0, \mathrm{Rx} 2, \mathrm{Rx} 4)$, i.e. the plot of its resistance is a straight line, and some "worse" (Rx1, Rx3). The exponents $n$ are summarised in table 4.2 
Table 4.2: Exponents found in the fit of the sensors electrical resistance - temperature dependence according to equation 4.3 .

\begin{tabular}{|c|c|}
\hline Sensor & Exponent $n$ \\
\hline Rx0 & 0.176 \\
Rx1 & 0.173 \\
Rx2 & 0.241 \\
Rx3 & 0.182 \\
Rx4 & 0.189 \\
\hline
\end{tabular}

give more flexibility in fitting. The fitting was done with help of a Matlab ${ }^{\circledR}$ tool. The result of a numerical procedure highly depends on a proper choice of an initial point. Two types of equations were tested: $T=a \exp (b / R)+c \exp (d / R)$ and $T=a \exp (b / R)+c \exp (d \cdot R)$. The one which resulted with a closer fit smaller RMSE was chosen.

Following an example of the reference LS sensor calibration curve, which is in the form of a Chebyshev polynomial, I decided to use the Chebyshev fit in the $300 \mathrm{mK}-3.6 \mathrm{~K}$ range. This polynomial works quite well in a broad temperature range, provided a sufficient amount of points is given, but only within the defined calibration limits. A recurrence formula is used to calculate the temperature 80:

$$
T=\sum_{i} A_{i} \cdot \cos (i \cdot \arccos (k))
$$

where $k=\left(\left(Z-Z_{L}\right)-\left(Z_{U}-Z\right)\right) /\left(Z_{U}-Z_{L}\right), Z=\log (R)$, and $Z_{U}$ and $Z_{L}$ are the upper and lower boundaries of the calibration range. Results of the calibration for the three bare chip sensors that were used in the measurement campaigns, i.e. $\mathrm{Rx} 0, \mathrm{Rx} 2$ and $\mathrm{Rx} 4$, in both temperature ranges are summed up in table 4.3 .

Later on, the ultra low temperature end of the calibration curves was verified with the SRD100 Fixed Point Device showing a satisfactory accuracy of the curves. The deviation between the calibration curve and the indication of SRD for sensor Rx4 at the three transition temperatures of $35 \mathrm{mK}, 64 \mathrm{mK}$ and $94 \mathrm{mK}$ are shown in table 4.4. The small discrepancy between the transition temperatures $T_{C}$ in table 4.1 and the temperatures given in table 4.4 is a results of keeping the sensors not perfectly in the middle of the transition during the calibration. 
Table 4.3: Calibration curves of the bare chip sensors labelled Rx0, Rx2, Rx4 in two temperature ranges.

\begin{tabular}{|c|c|c|c|}
\hline & $\mathrm{Rx} 0$ & $\mathrm{Rx} 2$ & $\mathrm{Rx} 4$ \\
\hline \multicolumn{3}{|c|}{ Exponential fit in the range 16 - 300 $\mathbf{~ m K}$} \\
\hline Equation & $\begin{array}{c}T=a \exp (b / R)) \\
+c \exp (d / R)\end{array}$ & $\begin{array}{c}T=a \exp (b / R) \\
+c \exp (d / R)\end{array}$ & $\begin{array}{c}T=a \exp (b / R) \\
+c \exp (d \cdot R)\end{array}$ \\
\hline a & -0.04961 & -0.114 & 0.04087 \\
b & 812.7 & 3269 & 5766 \\
c & 0.04267 & 0.1113 & -0.05737 \\
d & 5628 & 4788 & $-1.34 \mathrm{E}-05$ \\
\hline Chebyshev fit in the range 0.3 - 3.6 K (eq. & $\mathbf{4 . 4})$ \\
\hline Order & 11 & 10 & 10 \\
ZU & 3.463560 & 3.461210 & 3.472973 \\
ZL & 3.115042 & 3.118039 & 3.119736 \\
\hline \multicolumn{4}{|c|}{ Coefficients } \\
\hline A0 & 1.196255 & 1.242987 & 1.212532 \\
A1 & -1.386635 & -1.453824 & -1.418521 \\
A2 & 0.601521 & 0.598438 & 0.607825 \\
A3 & -0.261663 & -0.206505 & -0.249012 \\
A4 & 0.130127 & 0.066201 & 0.099380 \\
A5 & -0.048005 & -0.020462 & -0.025916 \\
A6 & -0.005229 & 0.006175 & -0.010027 \\
A7 & 0.022423 & -0.002558 & 0.014371 \\
A8 & -0.013987 & 0.001469 & -0.000447 \\
A9 & -0.003668 & -0.000300 & -0.008514 \\
A10 & 0.006139 & -0.002009 & 0.008002 \\
A11 & -0.007728 & & \\
\hline
\end{tabular}

Table 4.4: The accuracy of Rx4 calibration verified with the SRD1000.

\begin{tabular}{|c|c|c|}
\hline SRD1000 temperature & Deviation & Relative error \\
\hline $33.55 \mathrm{mK}$ & $0.31 \mathrm{mK}$ & $0.91 \%$ \\
$64.02 \mathrm{mK}$ & $-1.14 \mathrm{mK}$ & $-1.82 \%$ \\
$94.71 \mathrm{mK}$ & $-3.11 \mathrm{mK}$ & $-3.39 \%$ \\
\hline
\end{tabular}

\subsubsection{AA packages}

The calibration curve of the reference LS sensor, originally valid down to 50 $\mathrm{mK}$ in the form of a Chebyshev polynomial [80], was extended down to $20 \mathrm{mK}$ versus the SRD1000. Inverting Mott's law eq. (4.3) to obtain $T(R)$ dependence, 
we get

$$
T=T_{0}\left(\ln \frac{R}{R_{0}}\right)^{-1 / n} .
$$

This equation has 3 fitting parameters and it was applied to 3 or 4 temperature points of the SRD at around $20 \mathrm{mK}, 35 \mathrm{mK}, 63 \mathrm{mK}$ and $95 \mathrm{mK}$, as a precise low temperature extrapolation of the main fit. The reference sensor and all the others AA packages were placed on the base of the SRD1000 device in consecutive runs of the DR and calibrated. Results of the calibration for the reference LS sensor (extrapolation), Rx61 and Rx66 are presented in table 4.5.

Table 4.5: Summary of the low temperature calibration curves for the reference sensor and the two used AA packages in given ranges.

\begin{tabular}{|c|c|c|}
\hline Sensor & Equation & Range $(\mathrm{mK})$ \\
\hline Ref LS & $T=760\left(\ln \frac{R}{1327}\right)^{-1 / 0.4727}$ & $20-75$ \\
Rx61 & $T=32790\left(\ln \frac{R}{152.3}\right)^{-1 / 0.2718}$ & $30-100$ \\
Rx66 & $T=2300\left(\ln \frac{R}{592.1}\right)^{-1 / 0.4}$ & $30-250$ \\
\hline
\end{tabular}

\subsection{Estimation of the steady state measurement uncertainty at ultra-low temperatures}

\subsubsection{Temperature measurement uncertainty}

For low heating powers and very small temperature differences an accurate measurement of temperature is very important. The uncertainty of the temperature readout depends on the sensor used. For the reference LS sensor the absolute measurement error consists of the following three components:

- an absolute precision of the reference device used and the calibration done by the producer, defined as $\pm 3 \mathrm{mK}$ at $50 \mathrm{mK}, \pm 3.5 \mathrm{mK}$ at $100 \mathrm{mK}$ and $\pm 4 \mathrm{mK}$ at $300 \mathrm{mK}$ [90,

- the deviation of the Chebyshev fit obtained by the producer, which in a range of $50 \mathrm{mK}-0.8 \mathrm{~K}$ can be evaluated by the RMS error of the fit = $0.1 \mathrm{mK}$. Translated to the standard deviation as

$$
\sigma_{\text {fit }}=\sqrt{\frac{\text { fit points }}{\text { fit points }- \text { fit coefficients }}} \text { RMS , }
$$

it gives $\sigma_{\text {fit }}^{\mathrm{LS}}= \pm 0.16 \mathrm{mK}$,

- the standard deviation of the readout from the AVS resistance bridge.

For all the other sensors the absolute error consists of more components, e.g. for sensor $\mathrm{Rx} 4$ : 
- the inaccuracy of the reference LS sensor versus which they were calibrated, as described above:

- the precision of the reference device used by the producer 90,

- the perfection of the Chebyshev fit obtained by the producer $\sigma_{\mathrm{LS}}^{\mathrm{fit}}=$ $\pm 0.16 \mathrm{mK}$,

- the standard deviation of the readout from the AVS bridge, as described later;

- the perfection of the curve fit, which for Rx4 equals to $\sigma_{\mathrm{Rx} 4}^{\mathrm{fit}}= \pm 0.33 \mathrm{mK}$, and all the standard deviations for all the other sensors are summarized in table 4.6 ;

- the standard deviation of the $\mathrm{Rx} 4$ readout from the AVS resistance bridge, as explained below.

Table 4.6: Standard deviations of the calibration curve fits for the sensors used in the steady state measurements of the sandwich.

\begin{tabular}{|c|c|c|}
\hline Sensor & $\sigma_{\text {fit }}$ in $\mathrm{mK}$ & Range in $\mathrm{mK}$ \\
\hline Ref LS & 0.16 & $50-800$ \\
Rx61 & 0.4 & $30-100$ \\
Rx66 & 0.41 & $30-250$ \\
Rx4 & 0.33 & $30-300$ \\
\hline
\end{tabular}

The inaccuracy of the readout of an electrical resistance according to the manuals of the resistance bridges AVS 47A and AVS 47B [91] can be calculated as $\Delta R=0.001 \%$ FullScale $+0.05 \%$ Reading. A usual recording of a steady state measurement lasts around 10 - 20 min during which several tens of measurement points are created. Thus in the electrical resistance readout the standard deviation of the measured data has been included:

$$
\Delta R_{\mathrm{AVS}}^{\mathrm{el}}=\sqrt{(0.001 \% \text { FullScale }+0.05 \% \text { Reading })^{2}+\sigma_{\text {reading }}^{2}} .
$$

Converting it to the temperature difference:

$$
\Delta T^{\mathrm{AVS}}=\frac{T\left(R+\Delta R_{\mathrm{AVS}}^{\mathrm{el}}\right)-T\left(R-\Delta R_{\mathrm{AVS}}^{\mathrm{el}}\right)}{2} .
$$

An average $\Delta T_{\mathrm{LS}}^{\mathrm{AVS}}$ recorded during the calibration of sensors versus the reference LS equals to $\pm 0.12 \mathrm{mK}$.

Thus the total error in temperature for e.g. sensor $\mathrm{Rx} 4$ can be calculated as:

$$
\Delta T_{\mathrm{Rx} 4}^{\mathrm{tot}}=\sqrt{\left(\Delta T_{\mathrm{LS}}^{\mathrm{abs}}\right)^{2}+\left(\sigma_{\mathrm{LS}}^{\mathrm{fit}}\right) 2+\left(\Delta T_{\mathrm{LS}}^{\mathrm{AVS}}\right)^{2}+\left(\sigma_{\mathrm{Rx} 4}^{\mathrm{fit}}\right)^{2}+\left(\Delta T_{\mathrm{Rx} 4}^{\mathrm{AVS}}\right)^{2}} .
$$


The absolute error calculated according to this worst case scenario results in $\Delta T$ of approx $\pm 4 \mathrm{mK}$ at the low temperature end, and approx. $\pm 6 \mathrm{mK}$ at the high temperature end of the measurement, depending on the sensor.

\subsubsection{Power measurement uncertainty}

Voltage and current supplied to the heater are measured by two Keithley 2000 multimeters in four-wire configuration. According to their manuals, the precision of the readout is calculated as:

$$
\Delta U=40 \cdot 10^{-6} \cdot \text { Reading }+35 \cdot 10^{-6} \cdot \text { Range }
$$

for the voltage range below $100 \mathrm{mV}$,

$$
\Delta U=25 \cdot 10^{-6} \cdot \text { Reading }+7 \cdot 10^{-6} \cdot \text { Range }
$$

for the voltage range $100 \mathrm{mV}-1 \mathrm{~V}$,

$$
\Delta I=300 \cdot 10^{-6} \cdot \text { Reading }+80 \cdot 10^{-6} \cdot \text { Range }
$$

for the current measurement.

The error of power measurement equals to:

$$
\Delta \dot{Q}= \pm(|U \cdot \Delta I|+|I \cdot \Delta U|) .
$$

\subsubsection{Uncertainty of the interface thermal resistivity}

The resistivity error is calculated as:

$$
\begin{gathered}
R_{\max }^{\mathrm{th}}=\frac{\left(T_{\mathrm{Rx} 4}+\Delta T_{\mathrm{Rx} 4}\right)^{4}-\left(T_{\mathrm{LS}}-\Delta T_{\mathrm{LS}}\right)^{4}}{\dot{Q}-\Delta \dot{Q}} \frac{A_{\mathrm{max}}}{4} \\
R_{\min }^{\mathrm{th}}=\frac{\left(T_{\mathrm{Rx} 4}-\Delta T_{\mathrm{Rx} 4}\right)^{4}-\left(T_{\mathrm{LS}}+\Delta T_{\mathrm{LS}}\right)^{4}}{\dot{Q}+\Delta \dot{Q}} \frac{A_{\mathrm{min}}}{4} \\
\Delta R_{+}^{\mathrm{th}}=R_{\text {max }}^{\mathrm{th}}-R^{\mathrm{th}} \\
\Delta R_{-}^{\text {th }}=R^{\mathrm{th}}-R_{\text {min }}^{\mathrm{th}}
\end{gathered}
$$

A temperature uncertainty in the order of a few milli-Kelvin amplified by the power of four in eq. 4.14 and 4.15) would give an enormous error in thermal resistivity. It seems reasonable to assume that at zero heat load, the temperature difference between the measured elements of the sandwich setup should equal to zero as well. Thus we implement additional on the spot offset calibration, which cancels out the very small temperature difference caused by the remaining heat inleaks from wires, other residual heat loads of the sample 
environment or imperfect calibration of sensors. This offset is valid only close to the initial point with described conditions. It shifts the initial temperature of the stamp a little, reducing the temperature difference to zero and then decays quickly, without influencing the higher power and higher temperature part of the acquired data. An individual offset value was found for each base temperature of each configuration measured. It is therefore justified to assume that for the measurement of temperature differences at low heat loads some components of the total error $\Delta T^{\text {tot }}$ also cancel each other out. The temperature uncertainty could be reduced to the component caused by the resistance bridge, i.e. $\Delta T^{\mathrm{AVS}}$. The reduction of the error components decays in exactly the same way as the decay of the offset for each configuration and base temperature.

The presented approach is a very safe estimation of the possible error, rather overestimating, than underestimating it. A plot with error bars of the thermal interface resistivity for the first configuration measured - compressed setup in a normal conducting state is presented in figure 4.9 as an example.

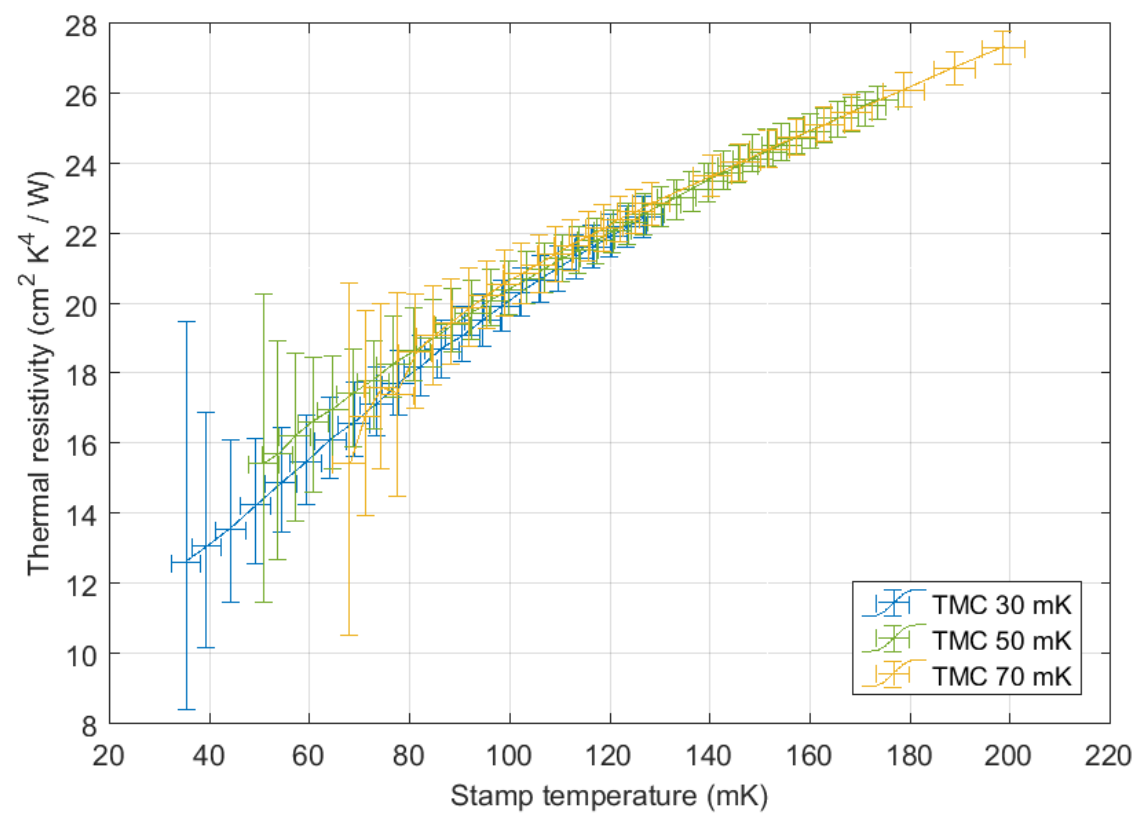

Figure 4.9: Example of a thermal interface resistivity curve for the compressed setup configuration with indium in the normal conducting state. Both horizontal error bars (for the stamp temperature), and vertical error bars (for the thermal resistivity) are presented. One can see that increasing the heat load and the temperature difference across the sample improves the accuracy. Blue - MC temperature $30 \mathrm{mK}$, green - MC temperature $50 \mathrm{mK}$, yellow - MC temperature $70 \mathrm{mK}$. 


\subsection{Transient measurement method}

\subsubsection{Overview of the measurement methods}

There are two basic ways to experimentally determine the thermal diffusivity and both of them are based on a 1D heat transfer equation with periodic, sinusoidal boundary condition. Application of a sinusoidal heating to one end of a semi-infinite rod, having basic physical properties like density $\rho$, heat capacity $c$ and thermal conductivity $\lambda$, results in the following time-dependent temperature distribution along its length $x$ :

$$
T(x, t)=T_{0}+T_{\mathrm{ampl}} \exp ^{-\frac{x}{\mu}} \cos \left(\omega t-\frac{x}{\mu}\right),
$$

where $T_{0}$ is the initial temperature, $T_{\mathrm{ampl}}$ is a maximum amplitude of the temperature oscilation at $x=0$, and $\mu=\sqrt{\frac{2 \lambda}{c \rho \omega}}$ is the thermal diffusion length [92]. In the first method, the temperature variation at a certain position $\mathrm{x}$ is measured and $\mu$ is calculated based on the attenuation of the temperature amplitude $T_{a m p l}(x)$ :

$$
\begin{gathered}
T_{a m p l}(x)=T_{a m p l}(0) \cdot \exp ^{-\frac{x}{\mu}}, \\
\mu_{\text {ampl }}=\frac{x}{\ln \left(T_{a m p l}(0) / T_{a m p l}(x)\right)} .
\end{gathered}
$$

In the second method, $\mu$ is evaluated from the phase shift $\phi(x)$ in the temperature signal:

$$
\begin{gathered}
\phi(x)=\frac{x}{\mu}, \\
\mu_{\text {phase }}=\frac{x}{\phi(x)-\phi(0)} .
\end{gathered}
$$

It can be a phase shift between the heater signal and the temperature sensor, or between two temperature sensors. In any case the thermal diffusivity $a$ is calculated as:

$$
a=\frac{\lambda}{\rho c}=\frac{\mu^{2} \omega}{2} .
$$

In the next sub-section the measurement chain used in these methods is analysed. Next, the accuracies obtained with each method are discussed and it is concluded that the phase shift methods are not accurate enough for our specific purposes. 


\subsubsection{Measurement chain analysis}

Voltages and currents used to read out the resistance of the temperature sensors at ultra-low temperatures are orders of magnitude lower than at room temperature. In the dilution refrigerator they should be low enough to avoid the self-heating of the sensor falsifying the measurements, but on the other hand high enough to make the signal well visible above the background noise. Picowatt AVS47A and AVS47B resistance bridges are used to read out the sensors keeping the power dissipated in the sensors at the level of femtowatts, and low pass filters reduce the noise in the signal. To make the measurements as fast as possible, the internal buffer of a Keithley 2000 multimeter is used to store the measurement data taken with a fixed frequency. In the transient measurements the signal from the temperature sensor passes through the:

- low-pass pi filter of the cryostat feedthrough,

- room temperature amplifier,

- resistance bridge,

- multimeter with an internal buffer, connected to the analog output of the resistance bridge,

and finally it is saved by the DAQ computer.

The power in the form of a sinusoidal wave of a given frequency and amplitude or a step function is supplied by a function generator. The signal also goes through a low pass filter, before it is stored in the buffer of the Keithley 2000 multimeter. There are elements present in the temperature read-out chain, like the amplifier and the resistance bridge, which are not present in the read-out of the power. It may be, that the difference in the measurement chains of the temperature and power, introduces an additional phase shift which obscures the real results. It is therefore necessary to validate the measurement capability of our DAQ, before applying the methods described in section 4.4.1 to measure the thermal diffusivity of the sandwich.

\subsubsection{Accuracy of the heater - temperature sensor phase shift method}

The frequency range of the thermal excitation is extended by comparison to previous measurements [4]. Several measurements for each frequency were made with the heater - temperature sensor phase shift method. The results were plotted, fitted and the phase shift was determined. For the diffusivity calculations a phase shift value in radians is used. The list of accuracies in seconds and radians as a function of the frequency of excitation is presented in table 4.7 72. The accuracy in radians decreases significantly for high frequencies $(>0.1 \mathrm{~Hz})$. 
Table 4.7: The accuracy of a heater - temperature sensor phase shift method as a function of frequency 72 .

\begin{tabular}{|c|c|c|}
\hline Frequency $(\mathrm{Hz})$ & Deviation $(\mathrm{s})$ & Deviation $(\mathrm{rad})$ \\
\hline $1 / 60$ & \pm 0.41 & \pm 0.05 \\
$1 / 30$ & \pm 0.15 & \pm 0.03 \\
$1 / 15$ & \pm 0.08 & \pm 0.04 \\
0.1 & \pm 0.03 & \pm 0.04 \\
0.2 & \pm 0.08 & \pm 0.21 \\
\hline
\end{tabular}

The influence of the resistance bridge on the phase shift was estimated by comparing the results obtained with and without the bridge present in the measurement chain at room temperature. The difference in phase shifts between the heater and the temperature sensor with and without the resistance bridge as a function of frequency is presented in figure 4.10 72. For low frequencies the introduced change of phase shift reaches 7 seconds. Such a big influence of the bridge on the results at low frequencies and the low accuracy of the measurement at high frequencies make the heater - temperature sensor phase shift method not suitable for the application in the DR setup.

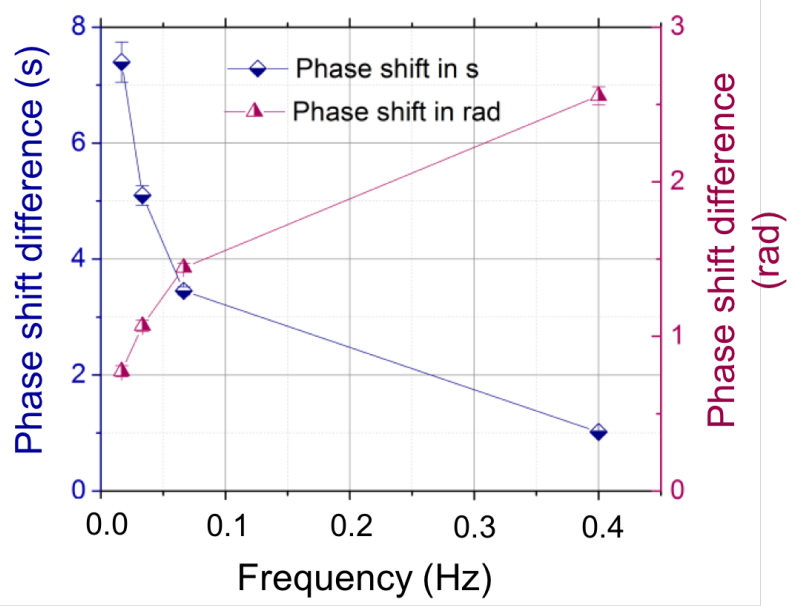

Figure 4.10: Differences in heater - sensor phase shifts between measurements with and without resistance bridge as a function of frequency 72 .

\subsubsection{Accuracy of the temperature sensor - temperature sensor phase shift method}

Having excluded the application of the heater - sensor phase shift method, one should analyse the accuracy of the sensor - sensor phase shift method. Signals of both of the sensors measuring the temperature difference across the sample go 
through the resistance bridge and are affected, but both in the same way. Thus the presence of the bridge should not influence the relative measurement of the phase shift. The two sensors are read out by the bridge in different resistance ranges of $20 \mathrm{kOhm}$ and $200 \mathrm{kOhm}$. The possible phase shift introduced by the resistance range setting on the bridge was therefore analysed [72], and the result is presented in figure 4.11. The accuracy of the phase shift caused by choice of the resistance range for the lowest frequency equals to $0.045 \mathrm{rad}$ and for the highest frequency to $0.29 \mathrm{rad}$. It could be used for measuring samples of low diffusivity, but highly diffusive samples like the sandwich setup require high excitation frequency (as explained in the next section) and therefore the accuracy of the sensor - sensor phase shift method is insufficient. This method will not be used for the sandwich setup measurements.

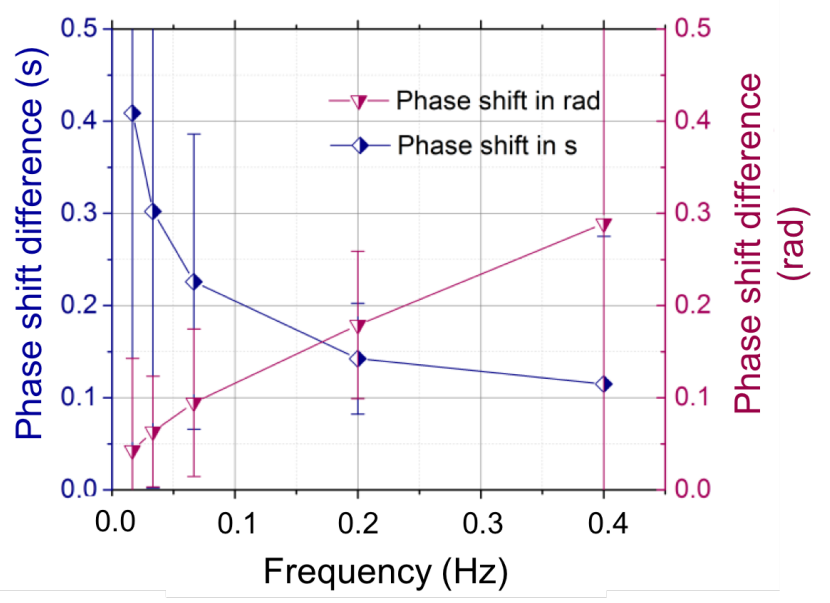

Figure 4.11: Differences in sensor - sensor phase shifts between measurements in resistance ranges $20 \mathrm{k} \Omega$ and $200 \mathrm{k} \Omega$ as a function of frequency 72 .

\subsubsection{Validation of the amplitude attenuation method and the threshold frequency.}

The amplitude method is not influenced by the measurement chain and settings of the resistance bridge, as both sensors are read out in the same way. The fast Fourier transformation (FFT) is used to extract the amplitude values from the high frequency measurement recordings. The validity of the amplitude attenuation method was verified on a copper rod sample at room temperature and at $\mathrm{LN}_{2}$ temperature by Jessica Golm 72 . The temperatures measured at two points at a well defined distance between each other were recorded with Pt100 sensors, and the diffusivity was calculated. An example of the recording for a frequency of $0.2 \mathrm{~Hz}$ is presented in figure 4.12 (left) and the obtained thermal diffusivity as a function of frequency in figure 4.12 (right). The cal- 
culated thermal diffusivity rises with the increase of frequency before reaching a plateau. Clearly, for very low frequencies the thermal response resembles "quasi-steady state", and no proper diffusivity can be measured. Only for frequencies higher than the critical value $f_{c}$ a constant value of diffusivity is found: $a=(1.2 \pm 0.2) \mathrm{cm}^{2} / \mathrm{s}$ at $T=297 \mathrm{~K}$ and $a=(3.2 \pm 0.3) \mathrm{cm}^{2} / \mathrm{s}$ at $T=77 \mathrm{~K}$ [72], which is in excellent agreement with literature values $a=1.15 \mathrm{~cm}^{2} / \mathrm{s}$ at $T=300 \mathrm{~K}$ and $a=3.1 \mathrm{~cm}^{2} / \mathrm{s}$ at $T=80 \mathrm{~K}[93$. The threshold value of frequency $f_{c}$ above which the plateau is reached, is increasing with the decrease of temperature: $f_{c} @ 300 \mathrm{~K}=0.06 \mathrm{~Hz}$ and $f_{c} @ 77 \mathrm{~K}=0.2 \mathrm{~Hz}$. One can expect that at ultra-low temperature the threshold frequency would be even higher.

The amplitude method is therefore suitable for the measurements of highly diffusive samples and will be used in the low temperature and ultra-low temperature study of the sandwich setups. Attention should be paid to the threshold frequency, which is increasing with a decreasing temperature, and it depends on the time constant of the sample and the thermalisation to the cooling source.
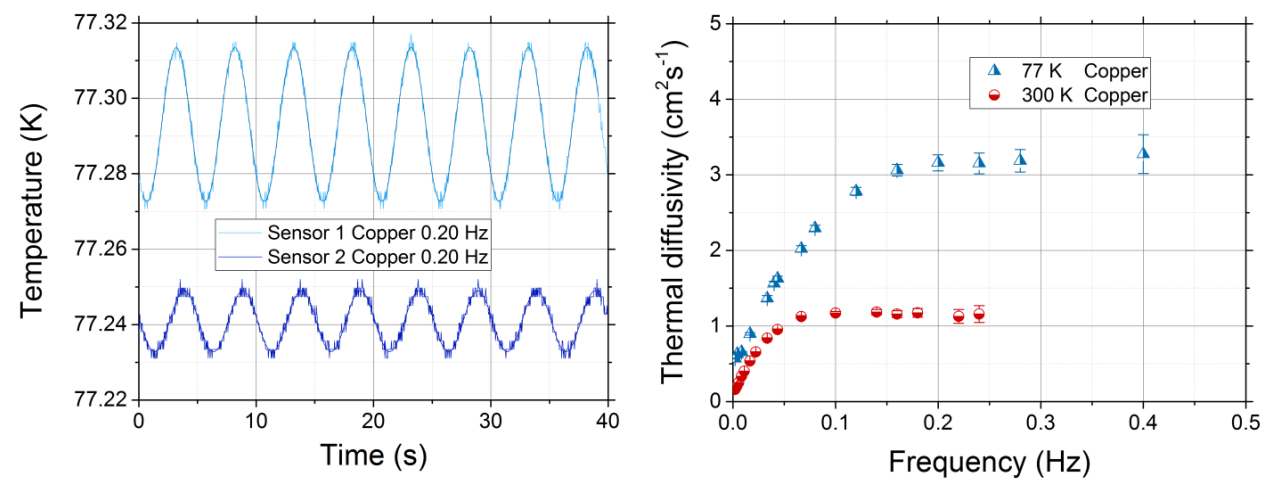

Figure 4.12: Left: a fragment of the temperature wave recording validating the amplitude method on a copper sample, for the heating frequency of $0.2 \mathrm{~Hz}$. Right: the thermal diffusivity of the copper sample as a function of the frequency of the applied heat wave. The diffusivity can be measured correctly only above a certain critical value of frequency $f_{c}$, that increases with a decrease of temperature, which is related to the thermal time constants of the sample and its thermalisation to the heat sink 72 .

\subsection{Estimation of the uncertainty in the low temperature ther- mal conductivity measurement}

The thermal conductivity of the sandwich setup in a higher temperature range was measured on the pulse-tube cryocooler with two LakeShore Cernox ${ }^{\circledR}$ sensors. The total error of the measurement on the cryocooler stand is a square root of the sum of squared errors in the measurements of the applied power, length, cross-sectional area and temperature gradient of the sample: 


$$
\Delta \lambda= \pm \sqrt{\left(\frac{\partial \lambda}{\partial \dot{Q}} \Delta \dot{Q}\right)^{2}+\left(\frac{\partial \lambda}{\partial L} \Delta L\right)^{2}+\left(\frac{\partial \lambda}{\partial A} \Delta A\right)^{2}+\left(\frac{\partial \lambda}{\partial T} \Delta T\right)^{2}}
$$

Calculating the partial derivatives we obtain:

$$
\begin{aligned}
\Delta \lambda= & \pm\left[\left(\frac{L}{A \cdot \Delta T} \Delta \dot{Q}\right)^{2}+\left(\frac{\dot{Q}}{A \cdot \Delta T} \Delta L\right)^{2}+\left(\frac{\dot{Q} \cdot L}{A^{2} \cdot \Delta T} \Delta A\right)^{2}\right. \\
& \left.+\left(\frac{\dot{Q} \cdot L}{A \cdot(\Delta T)^{2}} \Delta(\Delta T)\right)^{2}\right]^{1 / 2}
\end{aligned}
$$

where the error of the heat load measurement $\Delta \dot{Q}$ can be calculated as a square root of a sum of squares of the errors from the voltage $U$ and current $I$ measurements:

$$
\Delta \dot{Q}= \pm \sqrt{(I \Delta U)^{2}+(U \Delta I)^{2}} .
$$

The diameter of the sapphire disk equals to $\phi 20 \pm 0.1 \mathrm{~mm}$, which results in a cross-section of $A=3.14 \pm 0.03 \mathrm{~cm}^{2}$.

The distance between the stamp and platform sensors in the sapphire indium - copper setup equals to $L_{\mathrm{In}}=19.75 \pm 0.1 \mathrm{~mm}$ and for the sapphire titanium - gold - indium - copper setup equals to $L_{\mathrm{Ti}-\mathrm{Au}-\mathrm{In}}=27.75 \pm 0.1 \mathrm{~mm}$, so in both cases $\Delta L= \pm 0.1 \mathrm{~mm}$.

The resistance error depends on the voltage and current measurement deviation:

$$
\Delta R=\sqrt{\left(\frac{\partial R}{\partial U} \Delta U\right)^{2}+\left(\frac{\partial R}{\partial I} \Delta I\right)^{2}}=\sqrt{\left(\frac{1}{I} \Delta U\right)+\left(\frac{U}{I^{2}} \Delta I\right)^{2}}
$$

For both Cernox sensors the resistance is converted into temperature according to polynomial calibration fit curves in the following form:

$$
\log T=p_{0}+p_{1} \log (R)+p_{2}(\log (R))^{2}+\ldots+p_{11}(\log (R))^{11} .
$$

The absolute temperature measurement error is then calculated as:

$$
\Delta T=|T(R) \pm T(R \pm \Delta R)|
$$

what gives an error of approx. $\Delta T= \pm 7 \mathrm{mK}$ at $T=4.9 \mathrm{~K}$, and $\Delta T= \pm 116 \mathrm{mK}$ at $T=67 \mathrm{~K} 94$.

For the measurement of the temperature gradient along the sample, both sensors are read-out with the same current value supplied with the same current 
source, so the negligible error corresponding to the measurement of current is replaced with a standard deviation of the resistance:

$$
\Delta R=\sqrt{\left(\frac{1}{I} \Delta U\right)^{2}+(2 \sigma)^{2}} .
$$

Thanks to the offset compensation with zero heat load, the error between the two sensors is reduced to the differential measurement:

$$
\Delta\left(T_{1}-T_{2}\right)=\Delta T_{1}+\Delta T_{2} \approx 2 \Delta T,
$$

where $\Delta T$ is calculated from the resistance measurement as in eq. (4.29).

The error bars for the thermal conductivity measurements in the temperature range $3 \mathrm{~K}-60 \mathrm{~K}$ are usually within the size of a point on a plot. 


\section{Chapter 5}

\section{Mathematical model}

In order to design the electrode, a thermal model of the electrode is required. As discussed in chapter 3, two sandwich structures are used to develop and validate this model. The first structure consists of copper, indium and a sapphire disk, whereas the second resembles the actual electrode, configuration with layers of titanium and gold layers deposited on the sapphire disk. Both sandwich setups contain multiple layers and dielectric-metallic interfaces and they are therefore too complex to investigate the influence of each separate parameter by analysing the behaviour of the entire sandwich directly from the measurement observation. Mathematical modelling based on the available literature data is necessary to describe the observed phenomena in a way that would allow its application for other purposes. To simplify this difficult task, the setups are divided into smaller, easier to analyse fragments. The most basic sub-set, from which one can start the analysis, is the copper - indium - copper connection between the lid of the mixing chamber and the platform. The efficiency of electrons in transferring heat between normal conducting metals is so high, that the corresponding thermal resistance is not measurable within the required precision. Thus, the first analysed configuration will involve copper and superconducting indium, since the thermal interface resistance between copper and normal conducting indium is negligible.

The following configurations will be analysed in order of increasing complexity (here sc stands for superconducting, and nc for normal conducting):

1. copper - indium sc - copper

2. copper - indium nc - sapphire /symmetric

3. copper - indium sc - sapphire /symmetric

4. copper - indium nc - gold - titanium nc - sapphire /symmetric

5. copper - indium sc - gold - titanium sc - sapphire /symmetric 
Each case is simplified and presented as a series of resistors, where each resistor symbolizes the thermal resistance of a layer or an interface. Such a simplification of the setup by separation into multiple resistances in series allows to calculate the intermediate temperatures between the resistances by solving a system of independent one-dimensional equations. The Fourier equation describing the heat transfer can be simplified in the one-dimensional steady state case to the well known form:

$$
\dot{q}=A \lambda(T) \frac{d T}{d x},
$$

where $\dot{q}$ is the steady-state heat load, A is the area of the cross-section, and $\lambda$ is the temperature dependent thermal conductivity.

As explained in chapter 2 , the sub-Kelvin thermal conductivity of all layers can be described as $\lambda=\alpha T^{n}(\mathrm{~W} /(\mathrm{m} \cdot \mathrm{K}))$, where $n=1$ for the conductivity of metals, $n=3$ for the conductivity of dielectrics and superconductors, and $\alpha$ is a constant taken from literature. Then, the heat transfer equation for all types of resistors in the model has the form:

$$
\dot{q} d x=A \alpha T^{n} d T
$$

Integrating both sides of the equation from the cold end (c) to the hot end (h) of the layer that is considered:

$$
\begin{gathered}
\dot{q} \int_{c}^{h} d x=A \alpha \int_{c}^{h} T^{n} d T, \\
\dot{q}=\frac{A}{l} \alpha \frac{\left(T_{h}^{n+1}-T_{c}^{n+1}\right)}{n+1}
\end{gathered}
$$

where $l$ is the length of the layer cross-section between the hot and cold ends. When the temperature at one side of the resistor and the heat load are known, one can calculate the temperature at the other side in an explicit way, obtaining:

$$
T_{h}=\sqrt[2]{2 \frac{\dot{q}}{\alpha_{m}} \frac{l}{A}+T_{c}^{2}}
$$

for metallic layers, and

$$
T_{h}=\sqrt[4]{4 \frac{\dot{q}}{\alpha_{d}} \frac{l}{A}+T_{c}^{4}}
$$

for dielectric and superconducting metallic layers. The constant $\alpha_{m}$ has a unit of $\mathrm{W} /\left(\mathrm{m} \cdot \mathrm{K}^{2}\right)$ and $\alpha_{d}$ a unit of $\mathrm{W} /\left(\mathrm{m} \cdot \mathrm{K}^{4}\right)$.

For relatively small $\Delta T$ the thermal resistance of interfaces can be described according to eq. 2.21 as $R=\frac{A \Delta T}{\dot{Q}}=\kappa T^{-3}$, where $\kappa$ is a constant having the unit of $\mathrm{cm}^{2} \mathrm{~K}^{4} / \mathrm{W}$ and can be calculated as:

$$
\kappa=\frac{15 \hbar^{3} \rho_{1} v_{1}^{3}}{2 \pi^{2} k_{B}^{4} \rho_{2} v_{2}}
$$


where $\rho_{1}$ and $v_{1}$ are the density and the speed of sound of the material with higher acoustic impedance and $\rho_{2}$ and $v_{2}$ of the material with lower acoustic impedance. The heat transfer equation at the interface for large temperature differences with respect to the absolute temperature has the form of $73,78,95$ :

$$
\dot{Q}=\frac{A}{4 \kappa}\left(T_{h}^{4}-T_{c}^{4}\right) .
$$

Knowing the temperature on one side of the interface and the heat flow, one can calculate the temperature on the other side of the interface as:

$$
T_{h}=\sqrt[4]{4 \dot{Q} \frac{\kappa}{A}+T_{c}^{4}} .
$$

Equations (5.5), (5.6), (5.9) were implemented in the model separately for each layer and each interface. Starting from the bottom, i.e from the temperature of the lid or of the platform, temperatures of all the layers up to the stamp were evaluated for a given heat load. The measured stamp temperature from a configuration without the clamping structure measured in a wide temperature range is given here as a reference to test and justify the modelling assumptions. The detailed analysis of the experimental results is presented in chapter 6, along with the calculation of the interface thermal resistivities based on the model built here. It is worth mentioning that thanks to the integration over the temperature from the cold to warm side, this solution is not based on the assumption of small temperature gradients, what makes its results more exact at higher heat loads.

A correct value of the speed of sound is necessary to evaluate the interface resistivity $\kappa$ according to eq. (5.7). The three phonon branches (one longitudinal and two transverse) contribute to the heat transfer across the interface, and there are different speeds of sound associated with each of them, as presented in table 2.1. For a copper - liquid helium case, it is evident that one should take into account only the longitudinal branch, as it is the only one present in the liquid. For solid - solid interfaces according to the AMM, both longitudinal and transverse waves can be transmitted from one medium to the other, converting into corresponding longitudinal and transverse waves [47,51,53. Some authors 96 take into account only the transverse vibrations, with the argument that the transverse speed of sound has usually a lower value and differs less between various materials, what opens a wider channel for the heat transfer across the interface, compared to the longitudinal mode. The contribution of longitudinal modes is shown by Wang [65] and by Chen [97 to be diminishing with decreasing temperature in thin layers. Since all interfaces in both sandwich setups have at least one neighbouring thin layer, all the interface resistivities are calculated with transverse values of the speed of sound. The longitudinal and transverse modes in the model are presented symbolically as two independent parallel resistors in all the figures showing analogous electrical schemes corresponding to 
various configurations of the sandwich setup. Such a representation is of course a huge simplification, since the modes can convert to the other type, and it is only supposed to remind the reader about the existence of both modes.

Material properties used for the estimation of the theoretical interface resistance, i.e densities and corresponding speeds of sound, are taken from literature data summarized in table 2.1.

\subsection{Copper - indium sc - copper connection}

In building up the complete sandwich stack, the model starts with the analysis of the connection between the lid of the mixing chamber and the platform, which consists only of two interfaces of the same type $\mathrm{Cu}-\mathrm{In}$, and one metallic layer of indium in between. When indium is in the normal conducting state and the thermal contact is very good, the temperature difference between the lid and the platform is lower than the accuracy of the measurement. Therefore that case will not be discussed.

The behaviour of the $\mathrm{Cu}$ - In sc - Cu connection is simulated with three elements in series: the interface resistance, the $125 \mu \mathrm{m}$ layer of metallic but superconducting indium, and the interface again. The resistivity of all three components has a temperature dependence $R \propto T^{-3}$. The ultra-low temperature conductivity of indium from 38$]$ in the nc state equals to $\lambda_{\text {In nc }}=\alpha_{\mathrm{nc}} T(\mathrm{~W} / \mathrm{K} \mathrm{cm})$ where $\alpha_{\mathrm{nc}}=5.735 \mathrm{~W} /\left(\mathrm{K}^{2} \mathrm{~cm}\right)$. The ratio of nc to sc thermal conductivity from [98] equals to $\alpha_{\text {In nc }} / \alpha_{\text {In sc }}=64.6 / T^{2}$. Thus, in the sc state $\lambda_{\text {In sc }}=\alpha_{\text {sc }} T^{3}$ where $\alpha_{\mathrm{sc}}=0.0888 \mathrm{~W} /\left(\mathrm{K}^{4} \mathrm{~cm}\right)$. The resistivity $\kappa$ of the $\mathrm{Cu}-\mathrm{In}$ interface estimated according to eq. (5.7) and calculated taking the transverse speed of sound equals to $2.09 \mathrm{~cm}^{2} \mathrm{~K}^{4} / \mathrm{W}$.

The CAD model of the $\mathrm{Cu}$ - In - Cu connection and the analogous electrical scheme corresponding to it, with all the elements described, is presented in figure 5.1. The additional orange colored resistors represent the possible couplings of electrons to surface waves [51]. Such couplings would open a new channel for heat transfer across the interface with a temperature dependence $R \propto T^{-5}$.

In the mathematical model the temperatures are calculated from the cold side ( $T_{\text {lid }}$ taken as an input parameter) towards the warm end $\left(T_{\text {platform }}=T_{4}\right.$ being the result of the calculation). In between the limits, there are two more temperatures calculated: the bottom temperature of the indium layer $T_{2}$, and the top temperature of the indium layer $T_{3}$, as presented in figure 5.1. All the temperatures calculated from the model as a function of the applied heat load for the base temperature of $50 \mathrm{mK}$ are presented in figure 5.2. In the same figure also the measured platform temperature is shown. 


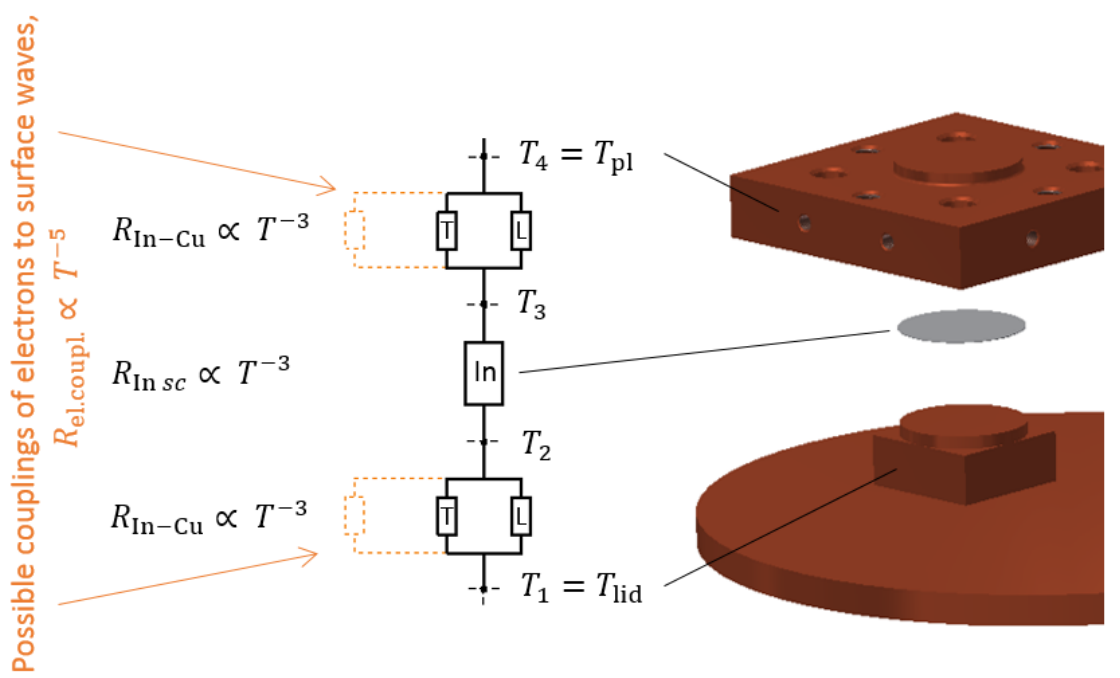

Figure 5.1: The CAD model of $\mathrm{Cu}$ - In - $\mathrm{Cu}$ connection between the MC lid and the platform with a scheme of the corresponding electrical model. The $\mathrm{T}$ and $\mathrm{L}$ resistors in parallel representing the interface resistance correspond to transverse and longitudinal waves. "In" resistor represents the indium layer.

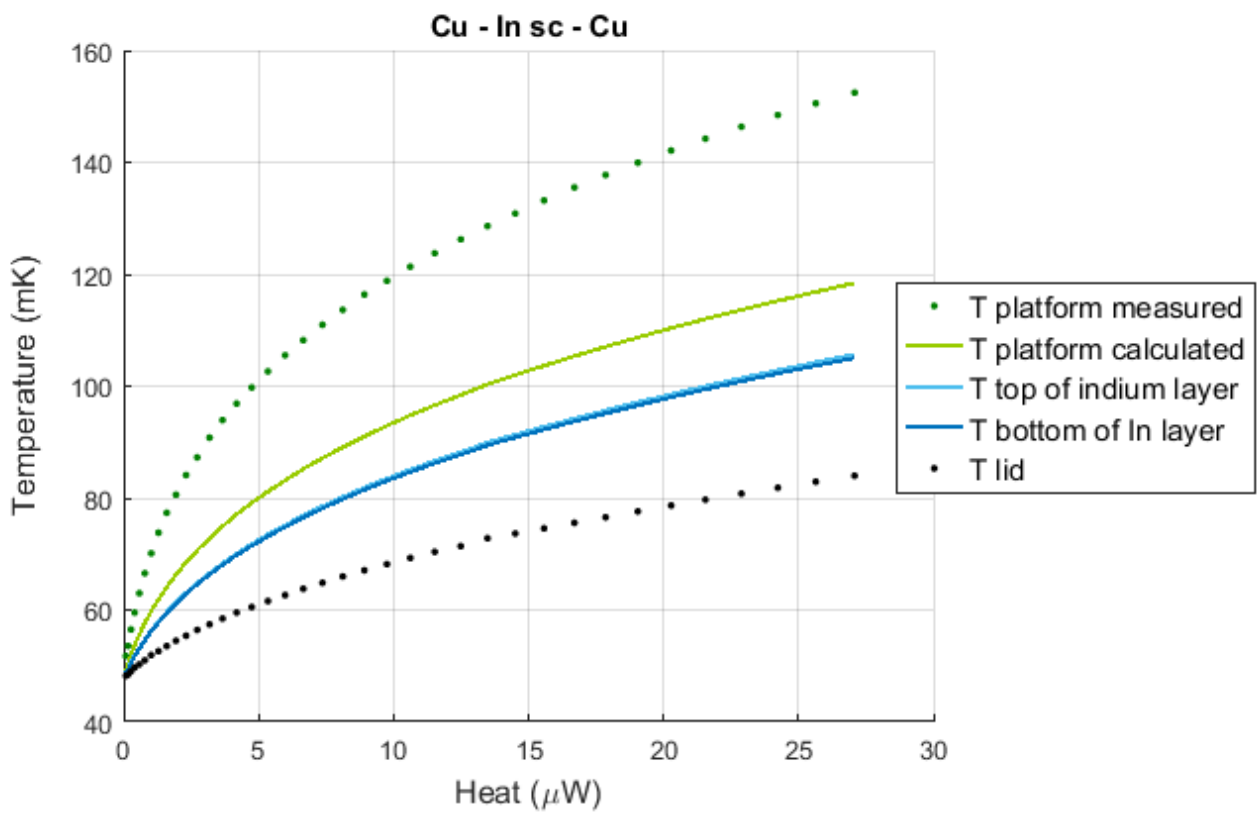

Figure 5.2: Measured lid and platform temperatures as a function of the heat load applied to the platform (bottom and top dotted lines, respectively), plus the calculated temperatures of the platform and across the indium layer, starting from the measured lid temperature (mixing chamber at $50 \mathrm{mK})$. 
One can see from the distances between the lines in fig. 5.2, that the main contribution to the total resistance comes from the interfaces rather than from the layer of indium, because the top and bottom temperatures of the indium layer are almost coincident. The calculated temperature of the platform is lower than the measured one, which means that the model underestimates the thermal resistivity of the $\mathrm{Cu}-\mathrm{In} \mathrm{sc}-\mathrm{Cu}$ connection. Therefore, a search for additional contributions to heat transfer in the form of electron couplings to surface waves is not opportune, since that will further reduce the interface resistance. The fact that both interfaces and the bulk thermal conductivity of the superconducting indium have the same temperature dependence $\propto T^{3}$ makes it difficult to estimate whether the additional resistance comes from the interfaces or from the layer of indium itself. A change of any of these parameters would result in the same overall effect. One can find in the literature more than one value of the speed of sound of indium $10,21,47,65,99$, , and as explained before, it is a subject of a discussion, if one should take into account longitudinal and/or transverse phonon waves. The interface resistance also depends heavily on the presence of oxides and quality of the interface. Thus the theoretical estimation of the interface resistance could vary within one order of magnitude. It seems reasonable to assume that the real resistivity of the interface is higher than predicted from the theoretical estimation.

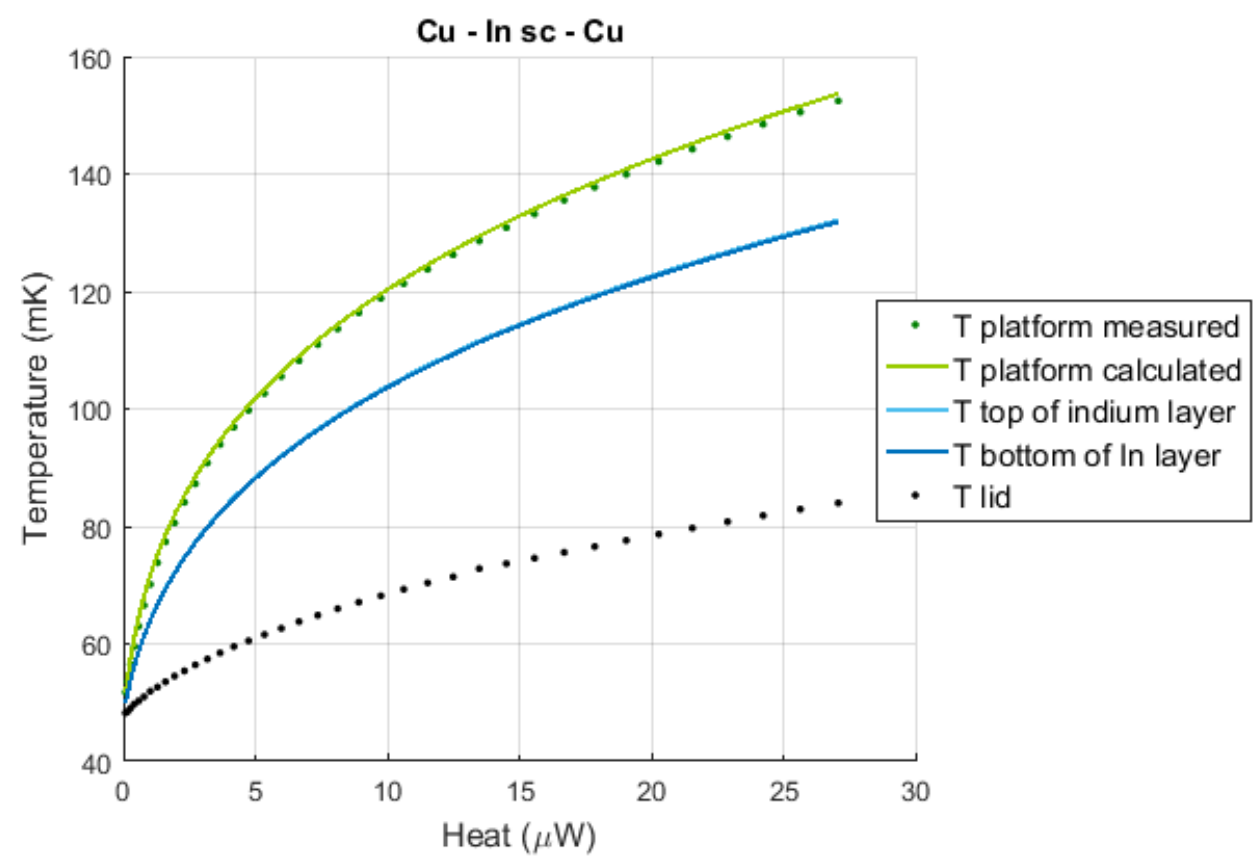

Figure 5.3: Temperatures starting from the measured lid temperature and calculated from the model with the theoretical $\mathrm{Cu}$ - In interface resistance multiplied by a factor of 3.5, as a function of the applied heat load for the mixing chamber temperature of $50 \mathrm{mK}$. 
Multiplication of the estimated interface resistance $\kappa_{\mathrm{Cu}-\mathrm{In}}$ by a factor of 3.5 , results in an almost perfect agreement of the measured and calculated temperature, see figure 5.3. The fact that only a multiplication of $\kappa$ resulted in a very good agreement of the measured and calculated data is also a proof that the temperature dependence $R \propto T^{-3}$ of the phenomenon has been correctly described in the model. Therefore the multiplication factor of 3.5 will be used in more complex configurations of the setup to describe the resistivity of the $\mathrm{Cu}$ - In connection.

\subsection{Copper - indium nc - sapphire connection}

The second analysed configuration is the copper - indium - sapphire sandwich with indium in the normal conducting state. Measurement of the $\mathrm{Cu}-\mathrm{In} \mathrm{nc}$ - $\mathrm{Cu}$ connection showed that the electrons are very efficient in transferring heat across the interface with In nc and also in normal conducting indium itself. Therefore in the $\mathrm{Cu}$ - In nc - sapphire setup, the main obstacle for the heat transfer is the indium - sapphire interface. Neglecting the contributions from the normal conducting indium, the electric analogy corresponding to this setup consists of three resistances in series: an indium nc - sapphire interface, the dielectric sapphire disk, and again a sapphire - indium interface (see figure 5.4.

The sub-Kelvin thermal conductivity of sapphire taken from 16 equals to: $\lambda_{\text {sapphire }}=\alpha_{\text {sapphire }} T^{3}(\mathrm{~W} /(\mathrm{cm} \mathrm{K}))$, where $\alpha_{\text {sapphire }}=0.023 \mathrm{~W} /\left(\mathrm{cm} \mathrm{K}^{4}\right)$. The sapphire - indium interface resistivity according to eq. (5.7) equals to $\kappa_{\text {sapphire-In }}=22.96 \mathrm{~cm}^{2} \mathrm{~K}^{4} / \mathrm{W}$. All three resistances in series have a theoretical $T^{-3}$ temperature dependence.

The resulting temperatures according to the model based on theoretical values are presented in figure 5.5. The total thermal resistance predicted by the model is higher than the measured one, and the main contribution comes from the interfaces, not from the sapphire itself.

The sapphire disk is made of a relatively perfect crystal and limited by flat, polished, parallel surfaces. At ultra low temperatures, one can assume that the phonons are not scattered significantly in the sapphire, and travel ballistically across the medium. Phonons that are within the cone of acceptance entering from on one side, remain in the same cone and directly exit on the other side. In an extreme case of no scattering at all in the sapphire, the two interfaces would behave like one. As stated by Peterson and Anderson [77] the total boundary resistance of the two interfaces could be described as:

$$
R_{\text {double int }}=(2-w) R_{\text {single int }}=(2-w) \kappa T^{-3},
$$

where $w$ takes a value from 0 (no parallel plates effect) to 1 (full effect). Application of a double interface resistance of that form in the model gives a 


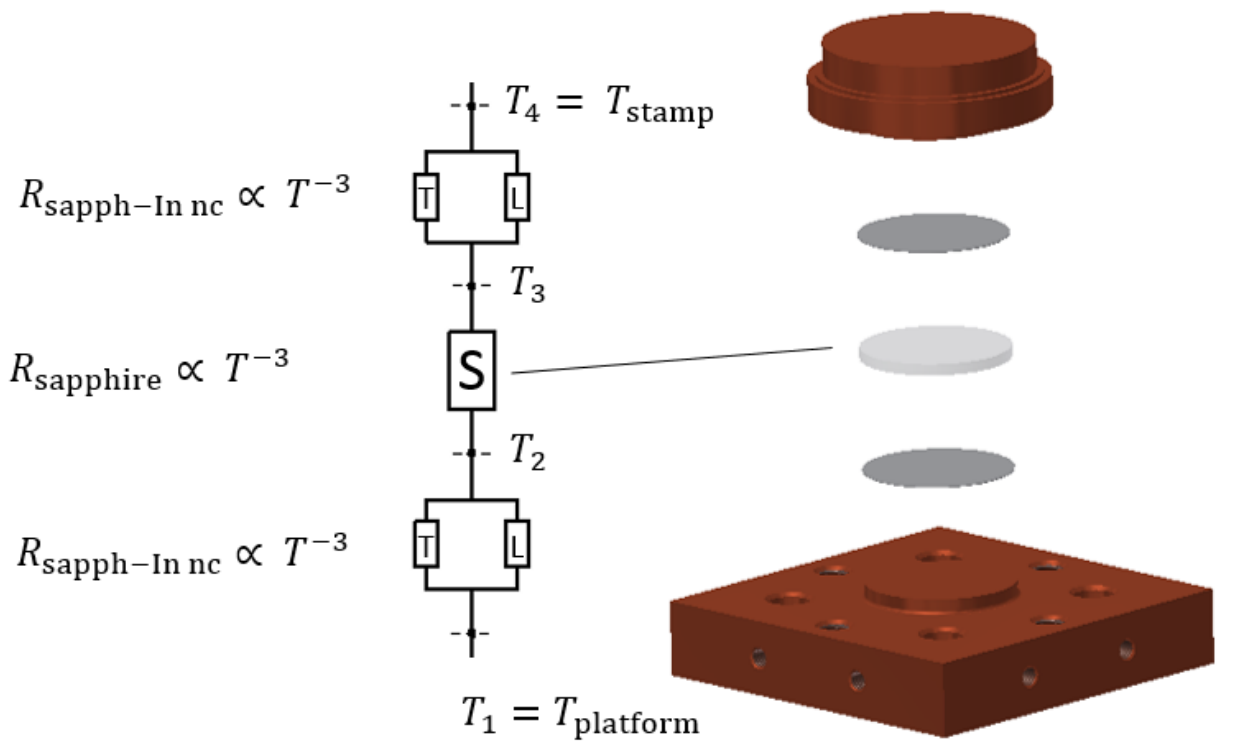

Figure 5.4: The $\mathrm{Cu}$ - In - sapphire connection between the stamp and the platform with the scheme of a corresponding electrical model with indium in the normal conducting state. The $\mathrm{T}$ and $\mathrm{L}$ resistors in parallel, representing the interface resistance, correspond to the transverse and longitudinal phonon waves. The " $\mathrm{S}$ " resistor represents the sapphire disk.

good agreement between the calculated and measured stamp temperature for $w=0.8$, as presented in figure 5.6 .

Certain small deviations between the measured and calculated values towards the high temperature end are consistent with the assumption that phonons are hardly scattered in the sapphire at ultra low temperatures, but the scattering becomes visible, although still very small, with the rise of temperature. The higher the temperature, the less accurate the parallel plates assumption is. That small discrepancy between measured and calculated values suggests that $w$ should in fact be temperature dependent to reflect the nature of the parallel plates assumption more accurately. The mean free path $l_{M F P}$ of phonon in sapphire can be calculated from the dependence between the thermal conductivity $\lambda$, heat capacity $c_{p}$, speed of sound $v_{s}$ and density $\rho$ described by equation $(2.9)$ :

$$
l_{M F P}=\frac{3 \lambda}{c_{p} \rho v_{s}} .
$$

It is usually assumed that the mean free path in dielectrics at ultra-low temperatures is so long, that both heat capacity and the thermal conductivity have a temperature dependence $\propto T^{3}$. Taking the ultra-low temperature thermal conductivity from [38] and assuming that the ultra-low temperature heat capacity of sapphire can be extrapolated from its low temperature heat capacity, tak- 


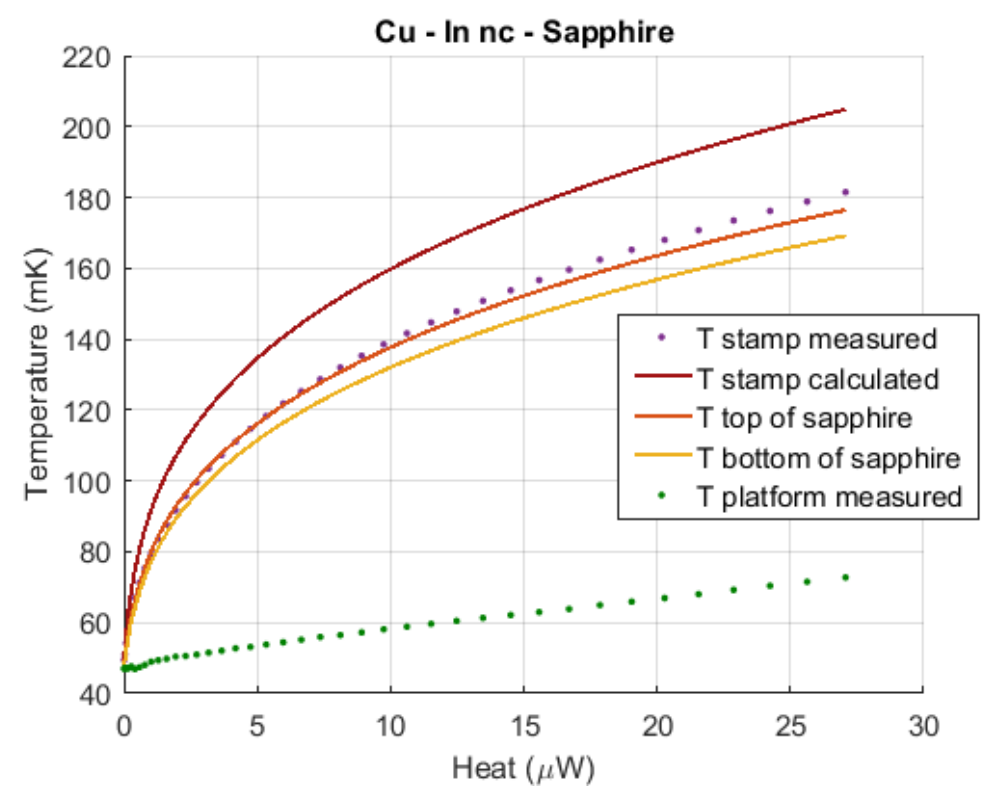

Figure 5.5: Temperatures starting from the measured platform temperature and calculated from the model based on the theoretical interface resistances as a function of the applied heat load for the base MC temperature of $50 \mathrm{mK}$.

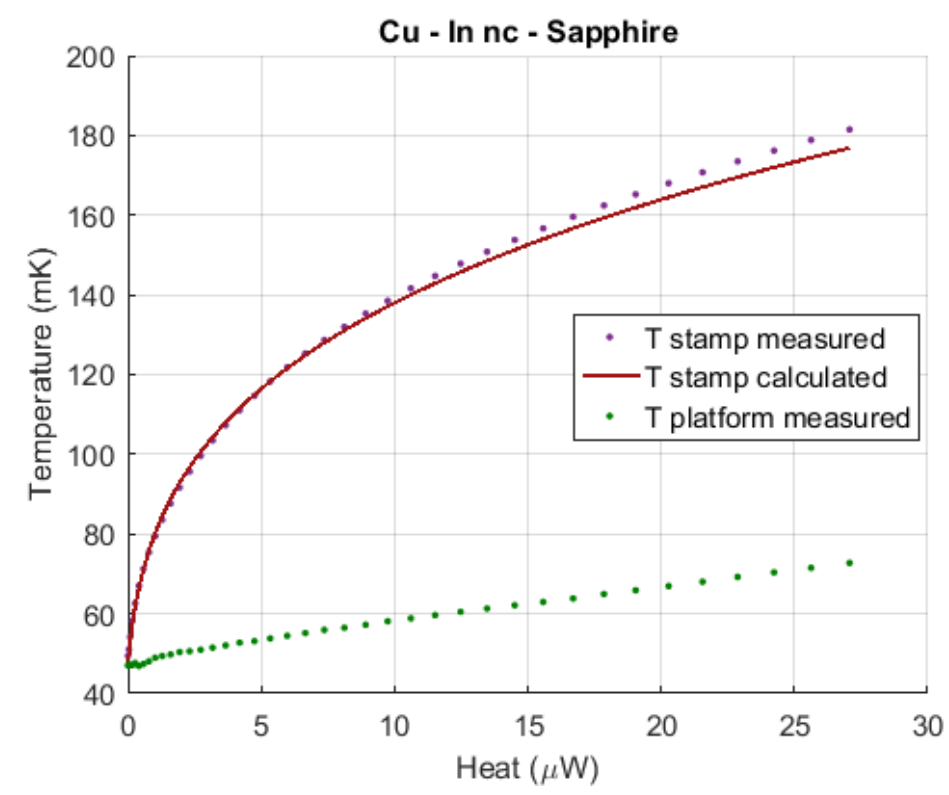

Figure 5.6: Temperatures starting from the measured platform temperature and calculated from the model including the parallel-plates assumption with $w=0.8$ as a function of the applied heat load for the base MC temperature of $50 \mathrm{mK}$. 
ing the same temperature dependence as ultra-low temperature properties of quartz, one can calculate the phonon mean free path in sapphire. Depending on the value of the speed of sound (longitudinal or transverse) the result changes a little, but it has a constant value in the order of $2-3 \mathrm{~mm}$ below $1 \mathrm{~K}$. The estimated value of the mean free path is 2-3 times larger than the thickness of the sapphire disk, therefore the parallel-plates assumption is valid.

A detailed comparison of the theoretical and experimental results is made in chapter 6 for the whole collected set of data.

\subsection{Copper - indium sc - sapphire connection}

Now the model can be extended to the full sandwich structure as presented in chapter 3 (see figure 3.1). The copper - indium interfaces were discussed in section 5.1 and the copper - indium - sapphire connection was considered in section 5.2 . In the latter case, indium was in the normal state and due to the high electron contribution to the thermal conductivity, the bulk thermal resistance could be neglected. In the practical case of the electrode, the indium is in the superconducting state which has two effects. Firstly, the electrons no longer contribute to the thermal conductivity. However, the resulting higher bulk resistivity can still be neglected. The overall thermal resistance is predominantly determined by the interfaces. Secondly, the interface resistance from sapphire to indium may also increase simillar to the increase of the copper - indium interface discussed in section 5.1. The CAD model of all the elements with the electric analogon of the resistors in series is presented in figure 5.7 .

The chain of resistors starts at the bottom at the temperature of the lid $T_{\text {lid }}=T_{1}$ and contains all the interfaces and layers, with the temperatures $T_{2}-T_{10}$ calculated in the nodes in between them, up to the temperature of the stamp $T_{\text {st }}=T_{11}$. Again, all the resistances have a temperature dependence $R \propto T^{-3}$. The additional orange color resistors represent possible couplings of electrons to surface waves [51]. Such couplings would open new channels for the heat transfer across the interfaces with a temperature dependence $R \propto T^{-5}$. According to [51] they could be present only if the electrons are available for the heat transport on the side with a higher speed of sound, which is true for the In sc - Cu interface, but not for the In sc - sapphire interface.

The indium - sapphire - indium connection is modelled including the parallelplates effect as concluded in the previous section $R_{\text {In-sapphire-In }}=(2-0.8)$. $22.96 T^{-3}=27.55 T^{-3}$. The $\mathrm{Cu}$ - In interface is modelled with a multiplication factor of 3.5 as a result obtained in section 5.1. $R_{\mathrm{Cu}-\mathrm{In}}=3.5 \cdot 2.09 T^{-3}=$ $7.32 T^{-3}$. The temperatures of the long chain of resistances are calculated explicitly and the result of this calculation is presented in figure 5.8 .

The calculated stamp temperature is much lower than the measured one, meaning that the model predicts lower resistivity values than they are in re- 


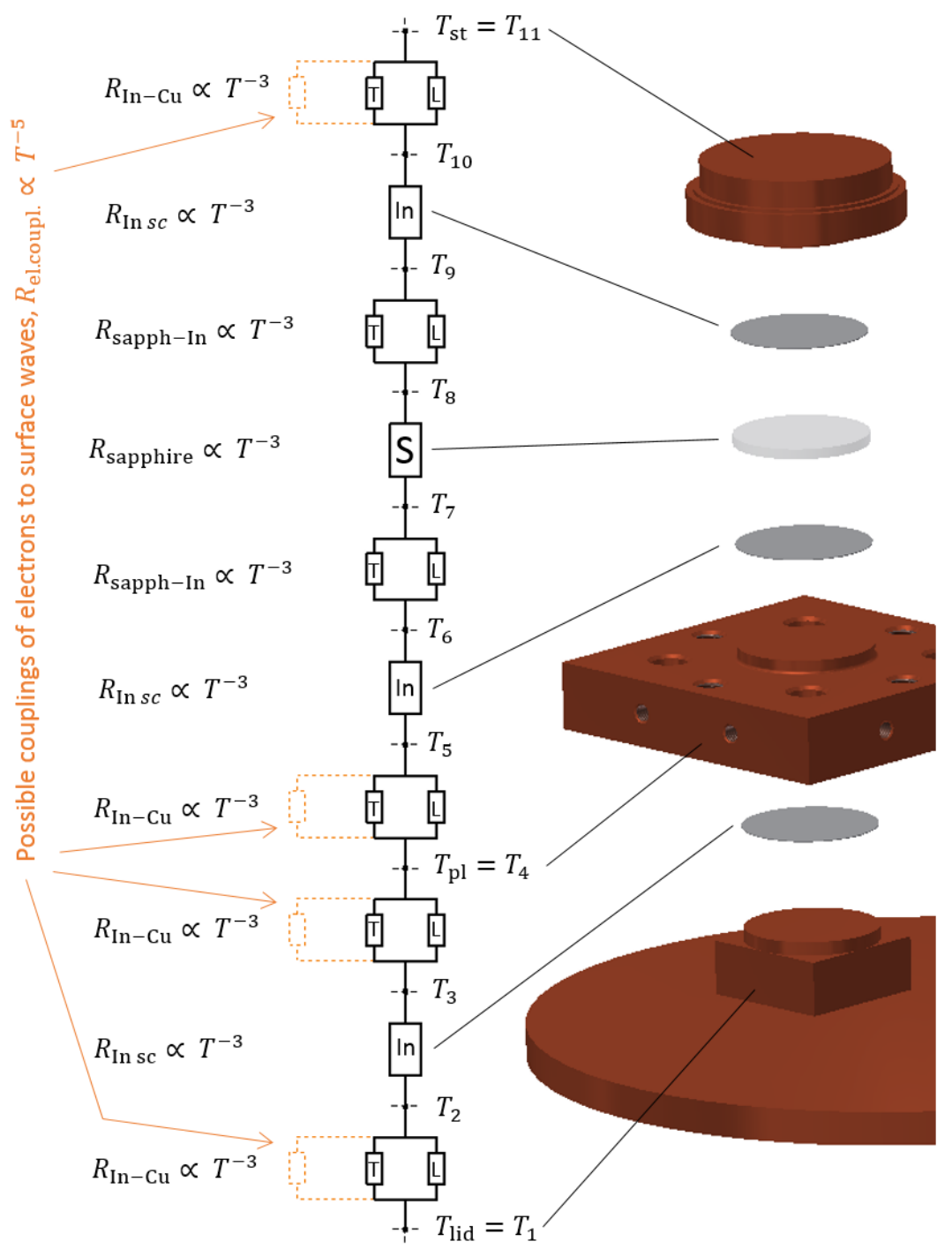

Figure 5.7: The $\mathrm{Cu}$ - In sc - sapphire /sym. setup with all the elements described. The electric analogy is a long series of resistors representing all the interfaces and layers. All the resistances have a temperature dependence $R \propto T^{-3}$. Orange coloured resistors symbolize possible couplings of electrons to surface waves with a temperature dependence $R_{\text {el.coupl. }} \propto$ $T^{-5}$, which could create an additional channel for the heat transfer.

ality. There could be two explanations of this discrepancy: either the indium - sapphire - indium connection with indium superconducting cannot be modelled as in the normal conducting case in section 5.2, or the $\mathrm{Cu}$ - In interfaces in the part between platform and stamp differ significantly from the interfaces analysed in section 5.1 .

The electrons in normal conducting indium could take part in the heat 


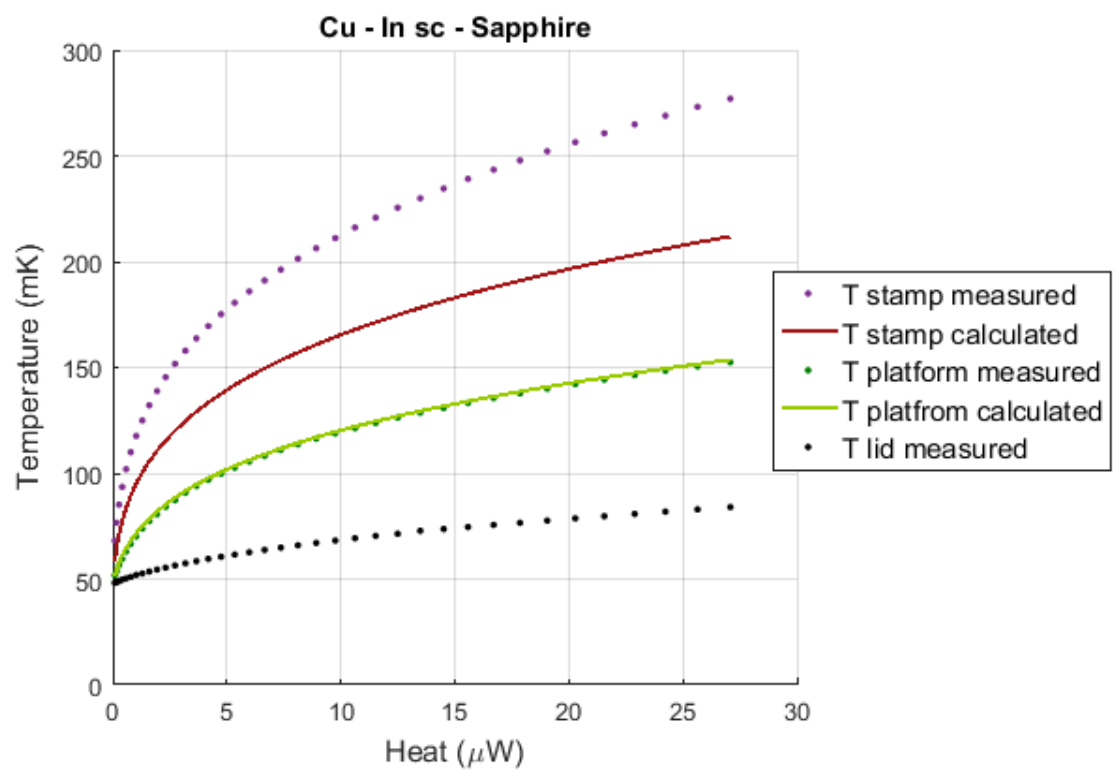

Figure 5.8: Dotted curves: measured temperatures of lid, platform and stamp as a function of heat load applied to the stamp for a MC base temperature of $50 \mathrm{mK}$. Solid lines: calculated platform and stamp temperatures, starting from the measured lid temperature. The theoretical value of the $\mathrm{Cu}$ - In sc interface resistivity has been multiplied by a factor of 3.5, according to the findings of section 5.1. The sapphire - In sc interface resistance included the parallel-plates assumption as a result from section 5.2 .

transfer across the interface by redistributing the energy over different phonon frequencies in indium, which is the acoustically weaker material. That would be contrary to the assumption of Papk and Narahara [51, but is not completely impossible, as they also measured a small change of the interface resistance depending on the state of indium (nc or sc). Multiplication of the sapphire - In interface resistance by a factor of 5 , results in a very good agreement between the measured and calculated stamp temperatures in that configuration, as presented in figure 5.9. It is very improbable that the parallel plates assumption loses its validity with an application of the external magnetic field, as it has no influence on the bulk properties of sapphire and on the ballistic propagation of phonons in this dielectric.

The other possibility is that the $\mathrm{Cu}$ - In interface quality of the sandwich section between platform and stamp differs significantly from the lid to platform connection analysed in section 5.1. The Kapitza resistivity is known to have low reproducibility and to differ significantly between interfaces depending on the roughness, purity, oxidation, etc. 21, 53, 77]. Despite the attempts to reproduce all $\mathrm{Cu}$ - In interfaces with the same procedure, the quality may differ between the interfaces. Moreover, the lid to platform connection was compressed much more than the sandwich itself. Higher compression should 


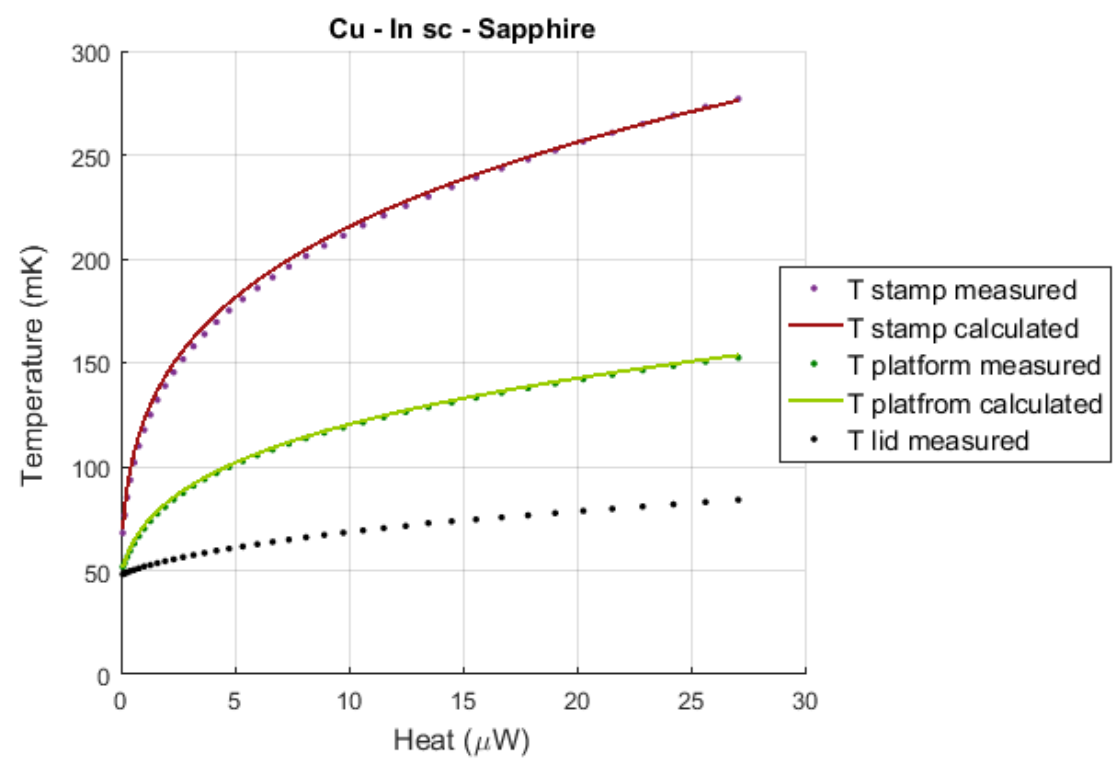

Figure 5.9: Dotted curves: measured temperatures of lid, platform and stamp as a function of heat load applied to the stamp for a MC base temperature of $50 \mathrm{mK}$. Solid lines: calculated platform and stamp temperatures, starting from the measured lid temperature. The theoretical value of the $\mathrm{Cu}$ - In sc interface resistivity has been multiplied by a factor of 3.5 , according to the findings of section 5.1 The sapphire - In sc interface resistance included the parallel-plates assumption as a result from section 5.2 and was subsequently multiplied by a factor of 5 .

make the indium creep more, and might have formed a better indium - copper bound than in the sandwich. Multiplication of the $\mathrm{Cu}$ - In interface by a factor of 28 (which seems a lot) gives a good agreement of the measured and calculated data comparable to fig. 5.9.

Multiplication of any of the resistivities $\kappa$ by an optimized constant value results in a good agreement between the measured and calculated temperatures. The different contributions cannot be discriminated since all resistances have a temperature dependence of $T^{-3}$. Therefore, an important conclusion should be drawn, that the temperature dependence of the observed phenomena is correctly described by the model. The resistance of the sandwich consisting of sapphire, indium and copper depends only on interfaces involving dielectric-like properties, and much less on a dielectric layer of sapphire or metallic indium. An analysis of the measurement results and its comparison with the model is presented in chapter 6 . 


\subsection{Copper - indium nc - gold - titanium nc - sapphire}

The second sandwich consisted of layers of copper, indium, gold, titanium and sapphire. The thin layers of gold and titanium are orders of magnitude thinner than indium or sapphire in the previous setup. The easier to analyse, i.e. containing fewer dielectric interfaces, is the case with both indium and titanium in the normal conducting state, and therefore this case will be analysed first. The CAD model of all the elements visible and with the corresponding chain of resistors of its electric analogon is presented in figure 5.10 .

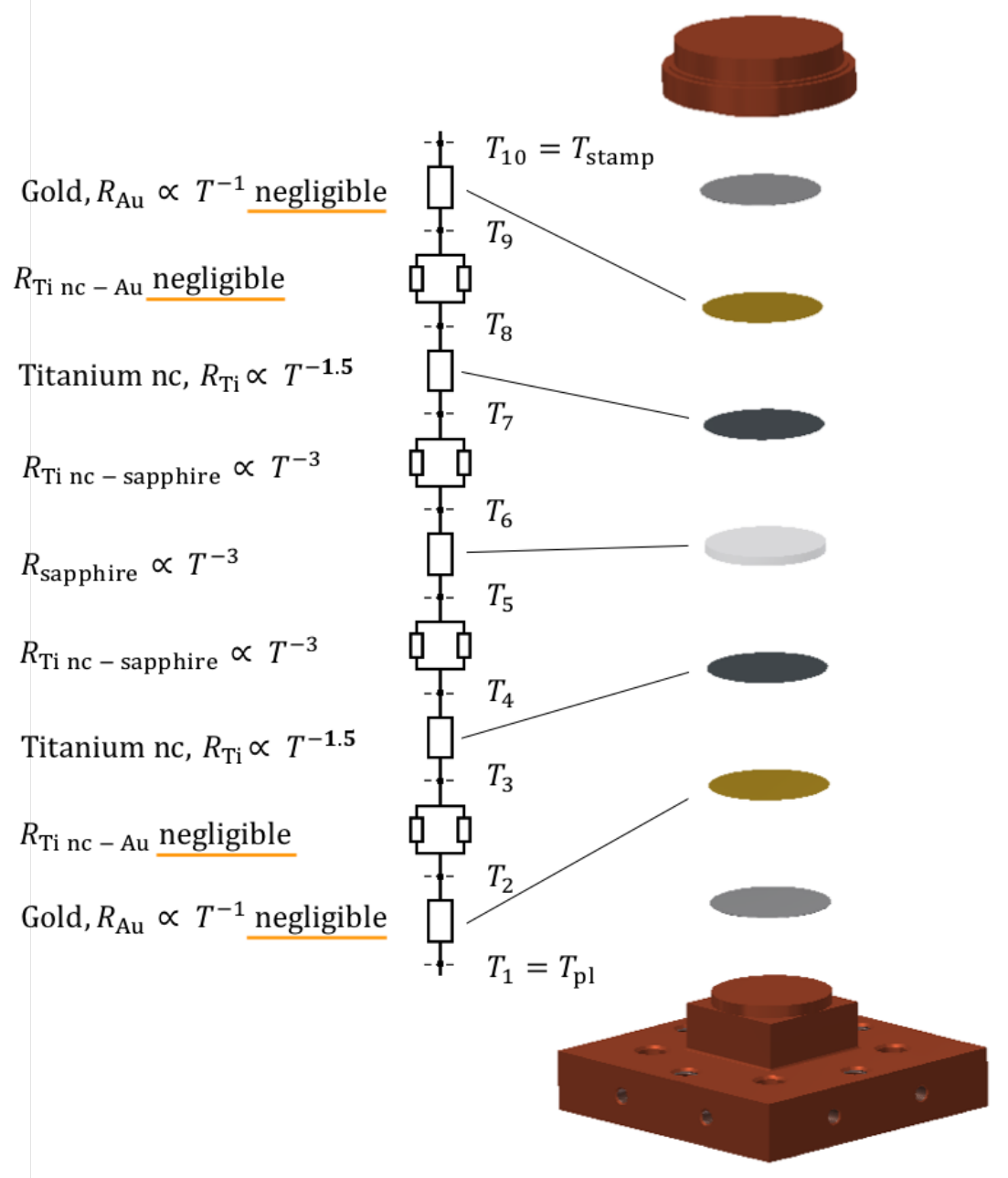

Figure 5.10: The CAD model of the $\mathrm{Cu}$ - In - $\mathrm{Au}$ - Ti - sapphire setup with all the elements visible. The corresponding electric scheme contains all the resistances used to calculate the total resistance of this setup with layers of indium and titanium in normal conducting state. Two resistors at every interface symbolize transverse and longitudinal phonons, but the final estimation of interface resistivity is calculated with transverse values of the speed of sound only. 
The ultra-low temperature thermal conductivity of gold equals to $\lambda_{\mathrm{Au}}=$ $\alpha_{\mathrm{Au}} T$, where $\alpha_{\mathrm{Au}}=4.31 \mathrm{~W} /\left(\mathrm{cm} \mathrm{K}^{2}\right)$ 33. The value depends mainly on the amount of impurities, which should be very small in a sputtered thin layer. However, its crystalline structure may influence the heat transport, see figures 2.15 and 3.5 . The low temperature thermal conductivity of titanium in the normal conducting state is taken from [34] $\lambda_{\mathrm{Ti} \mathrm{nc}}=3.45 \cdot 10^{-3} \mathrm{~T} \mathrm{~W} /(\mathrm{cm} \mathrm{K})$. In other sources [36 the lattice component of the thermal conductivity of normal conducting titanium in the $2 \mathrm{~K}$ to $30 \mathrm{~K}$ temperature range is evaluated to be equal to: $\lambda_{\mathrm{Ti} \mathrm{nc}}=1.8 \cdot 10^{-4} T^{1.5} \mathrm{~W} /(\mathrm{cm} \mathrm{K})$. The exponent higher than one shows that the lattice contribution to the overall thermal conductivity of titanium in normal conducting state is significant, even though the electrons are available for the heat transport. It may also be interpreted as a particularly weak electronic contribution to the total thermal conductivity, which would indicate that the thermal resistivity of the $45 \mathrm{~nm}$ Ti nc layer is not negligible.

The sapphire - titanium interface is the only interface involving dielectric properties in this configuration. The thermal boundary resistivity estimated with equation (5.7) for transverse values of the speed of sound gives a value of $\kappa_{\text {sapphire-Ti }}=18.27 \mathrm{~cm}^{2} \mathrm{~K}^{4} / \mathrm{W}$.

A series of equations corresponding to all resistors in the chain was solved and the computed temperatures along with the measurement data are presented in figure 5.11. Note that in this figure the measured lid and platform temperatures as well as the calculated platform temperature all coincide since the thermal resistance of the indium $\mathrm{nc}-\mathrm{Cu}$ interface can be neglected.

The temperature curves of bottom and top of the sapphire disk are drawn indicate that the main contribution to the total thermal resistance comes from the interfaces and not from the sapphire itself. The calculated temperature of the stamp is lower than the measured one, even without including the parallel plates assumption introduced in section 5.2. It may be that the mechanism of adhesion of Ti to the sapphire is disturbing the parallel plates assumption and a further reasoning for that effect will be discussed in the next chapter. A certain discrepancy between measured and calculated values could be an effect of an inaccurate value of the speed of sound. Multiplication of the sapphire - Ti interface resistivity $\kappa_{\text {sapphire-Ti }}$ by a factor of 1.5 , which seems a very reasonable and probable value, gives the calculated stamp temperature close to the measured one, but the temperature dependence, i.e the shape of the line is not represented adequately. The increase of thermal resistivity must come from another effect, characterised by a lower temperature dependence $T^{n}$, where $n \in(-3 ;-1)$. Such an exponent is present in the resistivity of gold and titanium $R \propto T^{-1}$. The thin layer of titanium deposited on the sapphire disk has a thickness of only $45 \mathrm{~nm}$ and as already mentioned, the bulk conductivity of titanium is lower than for other metals and has a trace of dielectric behaviour [36]. The thermal resistances of the titanium and gold layers present in the model vanish, because of their extremely small thickness, 


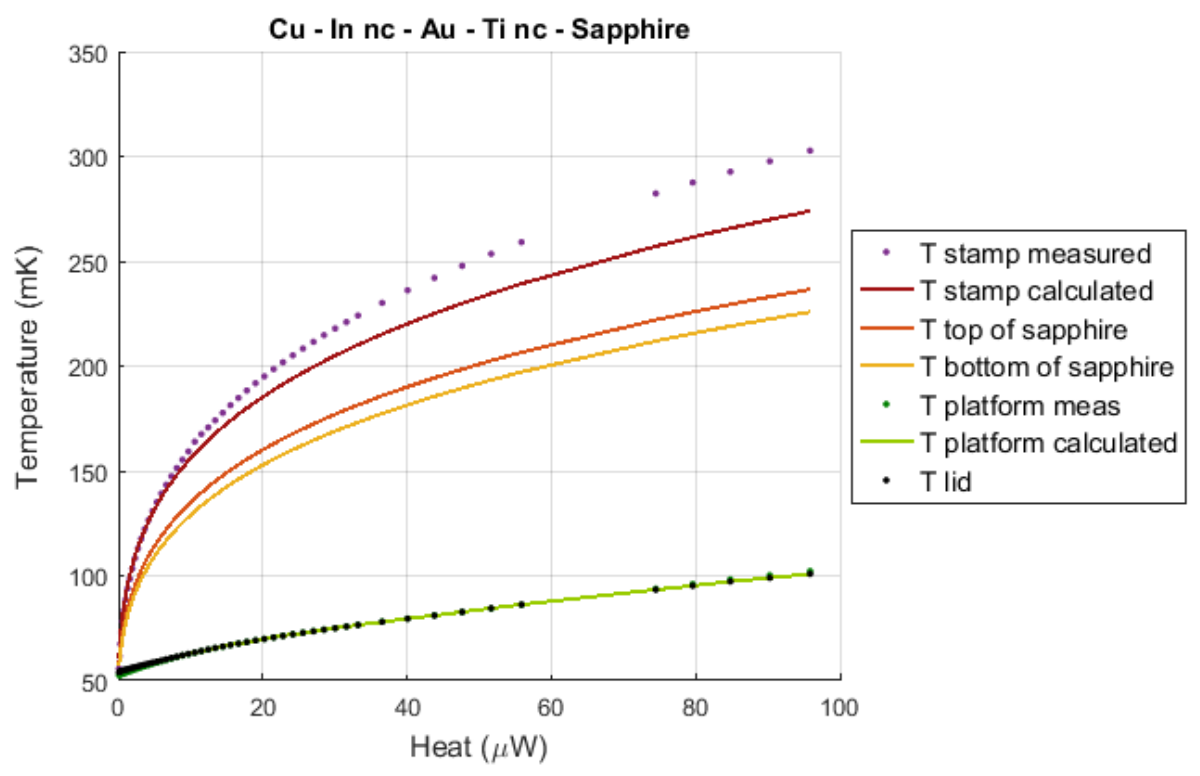

Figure 5.11: Dotted curves: measured temperatures of lid, platform and stamp in the $\mathrm{Cu}$ In nc - Au - Ti nc - sapphire setup as a function of heat applied to the stamp for a MC base temperature of $50 \mathrm{mK}$. Solid lines: calculated temperatures based on the model of figure 5.10 starting from the measured lid temperature.

when calculated in a classical way. At such low temperatures the mean free path of phonons exceeds the dimensions of the sample by far, the thermal gradient cannot be established, and the standard equation used for the heat transfer calculation does not hold. The effective thermal conductivity of such a layer is much lower as explained in section 2.5. A reduction of the apparent thermal conductivity of thin layers of various materials has been observed before and described in literature 60,61, 100 102. In the extreme case of the wavelength approaching or exceeding the dimensions of the sample, the effective thermal conductivity can decrease even several orders of magnitude (see figure 2.17). The dominant wavelengths of phonons in all the thin layers of the sandwich as a function of temperature are presented in figure 5.12. At ultra-low temperatures the wavelength associated with heat carriers in thin layers of gold and titanium is longer than the thicknesses of these layers. Gold is known to be a good conductor, both thermally and electrically, but the mobility of electrons in titanium is questionable [36], what creates a suspicion that the titanium layer can significantly decrease the overall thermal conductivity.

Treating the thin layer of titanium as a resistance having a temperature dependence given by low temperature properties of normal conducting titanium, but with an effective thermal conductivity severely diminished $\lambda_{\mathrm{Ti}}^{\text {eff }}=$ $6 \cdot 10^{-6} \lambda_{\text {Ti nc }}$ gives an excellent agreement between the measured and calculated temperature of the stamp, as presented in figure 5.13 . 


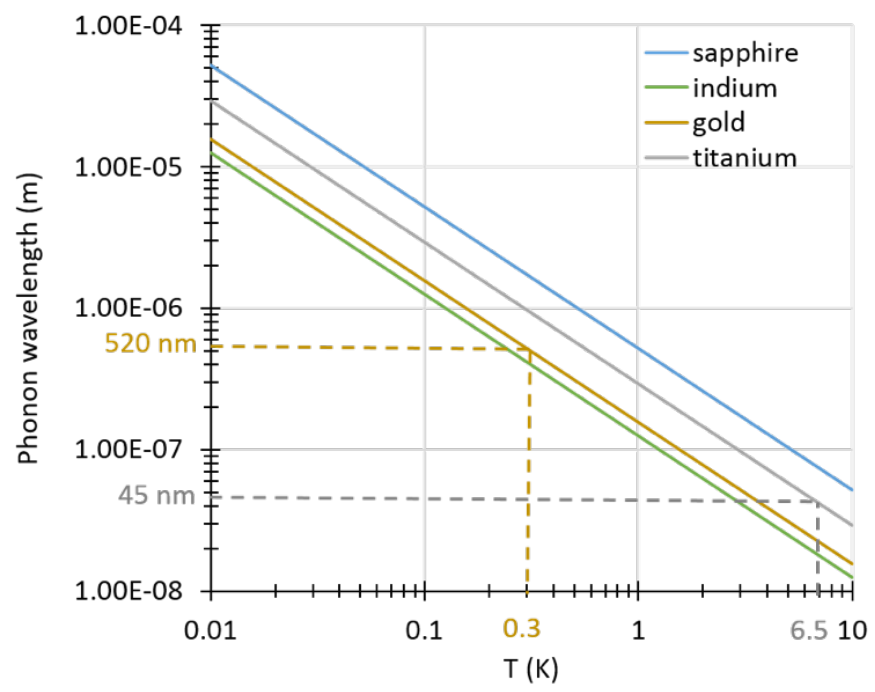

Figure 5.12: Phonon wavelengths in thin layers of the sandwich setup as a function of temperature. The wavelengths were calculated for the values of the speed of sound summarised in table 2.1. The temperatures at which the dominant phonon wavelengths in the thin layers of titanium and gold are equal to the thicknesses of these layers in the setup are marked.

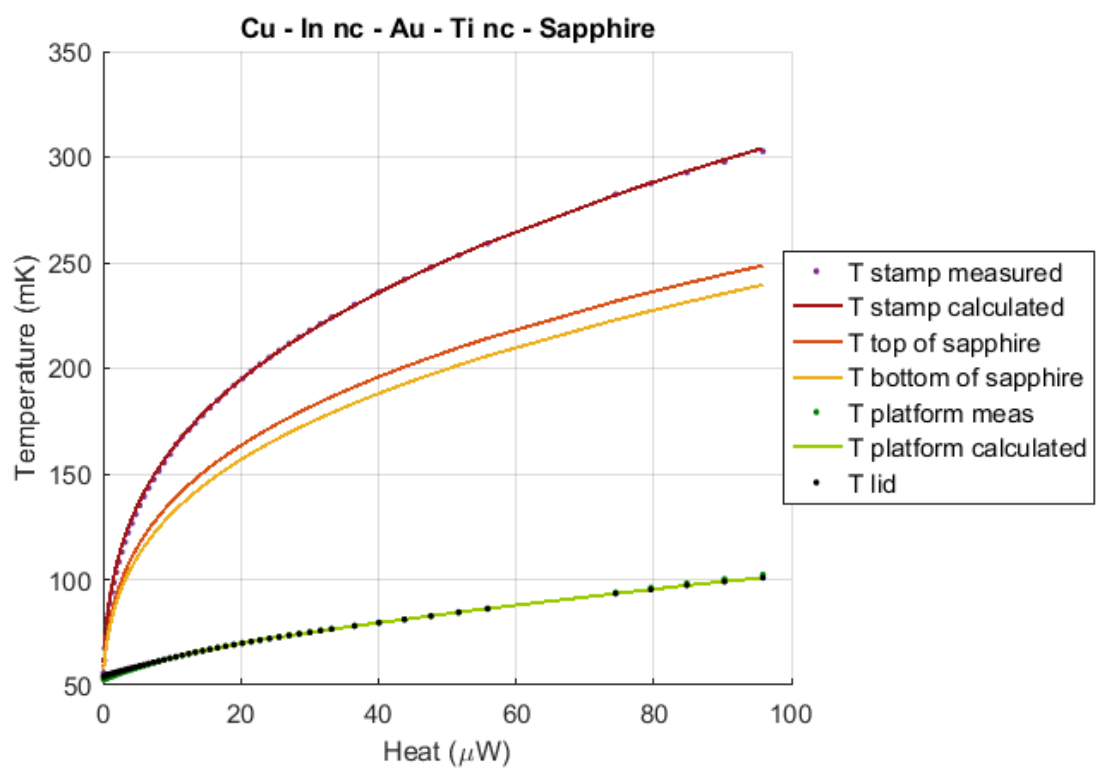

Figure 5.13: Dotted curves: measured temperatures of lid, platform and stamp in the $\mathrm{Cu}$ In nc - $\mathrm{Au}$ - $\mathrm{Ti} \mathrm{nc}$ - sapphire setup, as a function of heat applied to the stamp for a MC base temperature of $50 \mathrm{mK}$. Solid lines: calculated temperatures based on the model of figure 5.10 starting from the measured lid temperature. The theoretical conductivity of titanium was reduced several orders of magnitude to reflect the behaviour of the thin layer (multiplied by a factor of $6 \cdot 10^{-6}$ ). 
That agreement shows that the effect of reduced conductivity of thin layers dominates the heat transfer at ultra-low temperatures. The thin layer of titanium creates a serious bottleneck for the heat flow also in normal conducting state. A further confirmation of the importance and the influence of the thin layer of titanium will be given in the following section and in chapter 6 .

\subsection{Copper - indium sc - gold - titanium sc - sapphire connec- tion}

The last and the most complex configuration that remains, is the $\mathrm{Cu}$ - In $\mathrm{Au}$ - Ti - sapphire setup with indium and titanium in the superconducting state ( $\mathrm{Ti}$ is superconducting only below $0.39 \mathrm{~K}$ ). The multiple thin dielectric and metallic layers, and dielectric-metallic interfaces in series are presented in figure 5.14, along with the corresponding resistance chain of the electric analogon. As before, the resistance of all the interfaces is symbolized by two parallel resistors representing longitudinal and transverse phonon waves. The orange parallel resistors represent possible couplings of electrons to surface waves and are present only at two types of interfaces: In sc - $\mathrm{Cu}$ and $\mathrm{In} \mathrm{sc}-\mathrm{Au}$, i.e. in places where the conduction electrons are available on the acoustically "stronger" side [51].

Except for the thin layer of gold, which follows $R \propto T^{-1}$, the thermal resistances of all the other elements have temperature dependences $R \propto T^{-3}$. The thermal conductivity of the sapphire, indium and gold layers is the same as in previous sections. Ho and Hallock [98] defined the ratio of thermal conductances of indium in normal and superconducting states as $\lambda_{\text {In nc }} / \lambda_{\text {In sc }}=64.6 / T^{2}$. The same ratio is used to evaluate the low-temperature thermal conductivity of bulk superconducting titanium based on its normal conducting properties according to [34], obtaining $\lambda_{\mathrm{Ti} \mathrm{sc}}=5.34 \cdot 10^{-5} T^{3} \mathrm{~W} /(\mathrm{cm} \mathrm{K})$.

The copper - indium and sapphire - titanium interface thermal resistivity values were introduced in previous sections. The indium - gold and gold titanium interface thermal resistivity values calculated with transverse speed of sound are equal to $\kappa_{\mathrm{In}-\mathrm{Au}}=0.73 \mathrm{~cm}^{2} \mathrm{~K}^{4} / \mathrm{W}$ and $\kappa_{\mathrm{Au}-\mathrm{Ti}}=1.46 \mathrm{~cm}^{2} \mathrm{~K}^{4} / \mathrm{W}$, which is low compared to the sapphire - indium interface resistivity, due to relatively similar densities and speeds of sound in metals.

In section 5.1 it was shown that the $\mathrm{Cu}$ - In sc interface resistivity should be multiplied by 3.5 to reflect the measurement, and to account for the interface quality established between the stamp and the platform. The same multiplication is applied to the $\mathrm{Cu}$ - In interfaces in the present configuration of the setup.

As concluded in section 5.4 the thin layer of titanium has an enormous influence on the total resistivity of the sandwich. It can be expected that even a stronger influence is observed in a superconducting thin layer, when the 


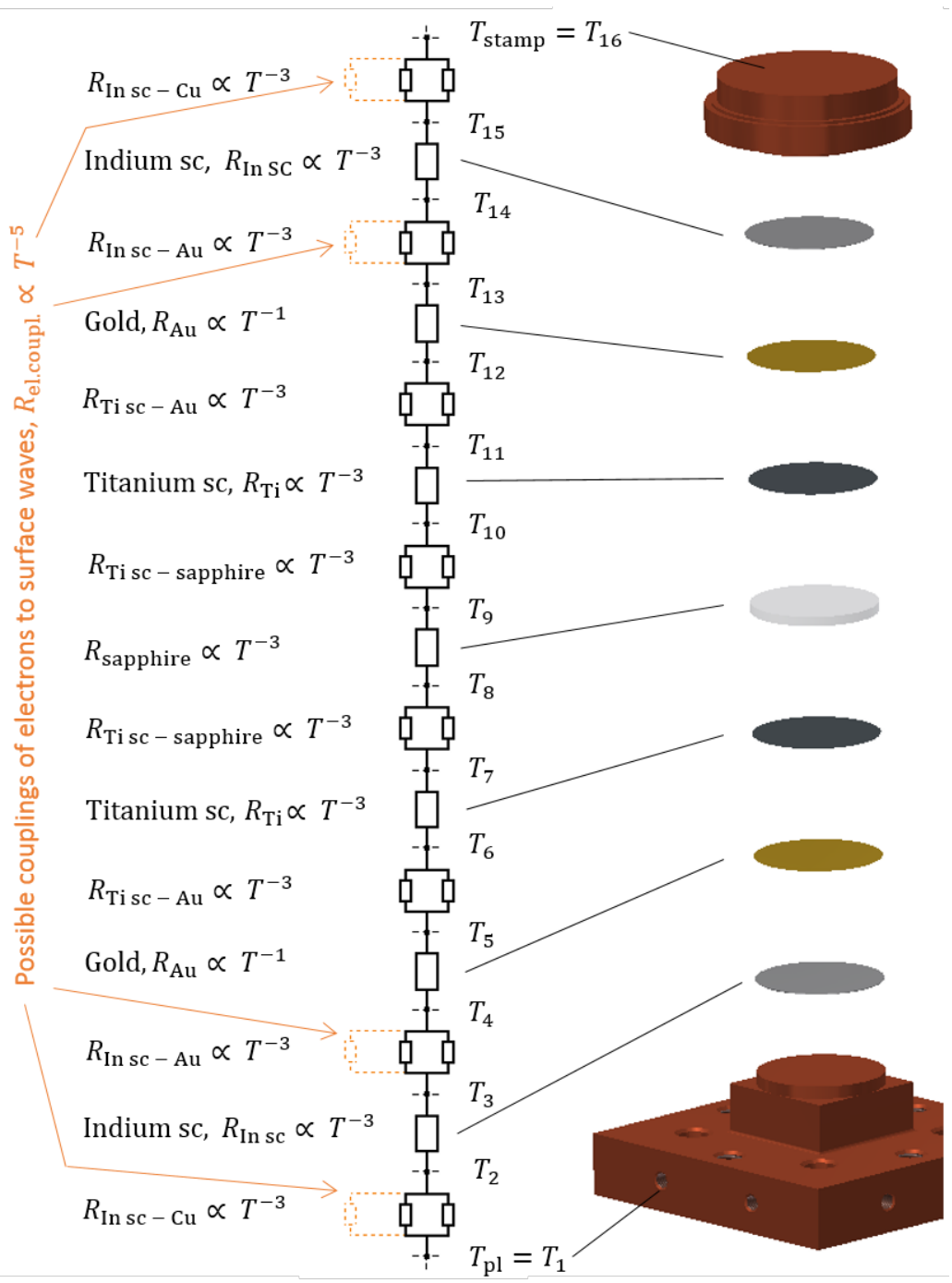

Figure 5.14: The CAD model of the $\mathrm{Cu}$ - In - $\mathrm{Au}$ - Ti - sapphire setup and its electric analogon. Indium and titanium ( $\mathrm{Ti}$ below $0.39 \mathrm{~K}$ ) are in the superconducting state and therefore the chain of resistors representing dielectric - metallic interfaces and thin layers in the electric analogon is particularly long. The orange parallel resistors represent possible couplings of electrons to surface waves and are present only at two types of interfaces: In sc - Cu and In sc - $\mathrm{Au}$, i.e. in places where the conduction electrons are available on the acoustically "stronger" side 51 .

electrons are not contributing to the heat transport at all, and the wavelength of phonons at ultra-low temperature exceeds the thickness of the layer (fig. 5.12 . A reduction of the effective thermal conductivity of the thin layer even by several orders of magnitude could be justified [65]. For the effective thermal conductivity of $\mathrm{Ti}$ sc $\lambda_{\mathrm{Ti} \text { sc }}^{\mathrm{eff}}=2.2 \cdot 10^{-4} \lambda_{\mathrm{Ti} \text { sc }}$ the calculated value of the 
stamp temperature agrees very well with the measured one as presented in figure 5.15. The reduction factor $2.2 \cdot 10^{-4}$ applied in the superconducting case is two orders of magnitude less severe than the factor $6 \cdot 10^{-6}$ introduced in section 5.4. The two orders of magnitude are result from applying the ratio $\lambda_{\text {Ti nc }} / \lambda_{\text {Ti sc }}=64.6 / T^{2}$ based on indium as discussed above. In reality the ratio $\lambda_{\mathrm{nc}} / \lambda_{\mathrm{sc}}$ for titanium is probably lower than for indium, because of a rather weak contribution of electrons to the thermal conductivity of normal conducting titanium. Keeping the reduction factor of $6 \cdot 10^{-6}$ obtained for the normal conducting state, without implementation of the nc/sc conductivity ratio 64.6 characteristic for indium, and only changing the temperature dependence from $\lambda_{\mathrm{nc}} \propto T^{1}$ to $\lambda_{\mathrm{sc}} \propto T^{3}$, the conductivity of titanium in the superconducting state would still have to be reduced 1.76 times to match the experimental results. It means that in reality the ratio of the nc to sc thermal conductivity of titanium close to its critical temperature equals to $\lambda_{\mathrm{Ti} \mathrm{nc}} / \lambda_{\mathrm{Ti} \mathrm{sc}}=1.76 / T^{2}$, which is much lower than for indium and it fits the picture of low thermal performance of titanium.

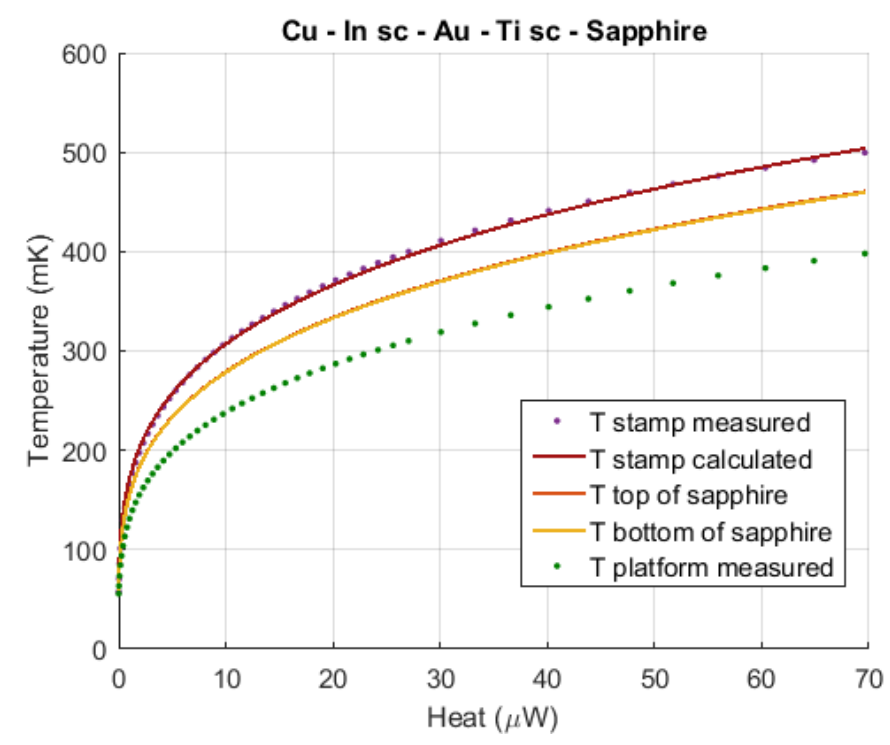

Figure 5.15: Measured and calculated temperatures of the $\mathrm{Cu}$ - In sc - Au - Ti sc - sapphire setup as a function of the applied heat load for the base MC temperature of $50 \mathrm{mK}$. To reflect the behaviour of the thin layer of titanium its bulk conductivity was multiplied by a factor of $2.2 \cdot 10^{-4}$ to get an almost perfect fit between measured and calculated stamp temperature.

Since most resistances have a temperature dependence $R \propto T^{-3}$ it is arguable if the increase of the total thermal resistance of the sandwich does not come from another effect, than only from the reduced thermal conductivity of a superconducting thin layer of Ti. The sapphire - Ti interface has been analysed in section 5.4, where it was demonstrated that the effect of thin layer dominates the conductivity of the whole sandwich. The couplings of electrons to surface 
waves should not be present, as sapphire is acoustically stronger than titanium. The quality of the sapphire - Ti interface should not change with an external magnetic field, so we can exclude it form the list of potential contributors to the total resistivity of the present configuration of the sandwich. The $\mathrm{Cu}-\mathrm{In}$ interface was modelled based on the findings from section 5.1, but it may be that its resistivity differs from the one in section 5.1 because of the smaller compression force applied to the sandwich compared to the lid - platform connection or because of eventual trace quantities of oxides. The other metallic interfaces: In-Au and $\mathrm{Au}-\mathrm{Ti}$ might have also created an intermetallic layer that could increase the total thermal resistivity, but its exact value is difficult to evaluate.

The main conclusion that should be drawn from the presented modelling is that the thin layer of titanium in both normal and superconducting state can have a huge effect on the total thermal resistivity of the setup. All the effects will be further analysed and evaluated in the next chapter. 
5. Mathematical model 


\section{Chapter 6}

\section{Measurement results}

There were several measurement campaigns conducted in the Cryolab DR, during which steady state and transient measurements were performed. During the measurements the heat was applied to the stamp and the temperatures on the stamp and on the platform were measured. The thermal resistivity was calculated according to equation 4.1, because of a relatively large temperature gradient across the sandwich structure compared to the absolute temperature. The detailed measurement methodology is described in chapter 4. The results obtained for the less complex setup with a sapphire - indium - copper sandwich are presented first, and the more complex analysis of a sapphire - titanium gold - indium - copper sandwich follows.

\subsection{Steady state measurements of the copper - indium - sap- phire sandwich setup}

In the first run of the DR the copper - indium - sapphire sandwich setup was studied in three different cases:

- sandwich compressed with a mechanical force in the MC temperature range $30 \mathrm{mK}-70 \mathrm{mK}$,

- sandwich without compression in the MC temperature range $30 \mathrm{mK}$ $100 \mathrm{mK}$,

- sandwich without the clamping structure in the MC temperature range $30 \mathrm{mK}-100 \mathrm{mK}$, later repeated in a wider MC temperature range $30 \mathrm{mK}$ $-300 \mathrm{mK}$.

Indium becomes superconducting at $3.41 \mathrm{~K}$ in the absence of an external magnetic field and at this temperature its thermal conductivity starts decreasing significantly with decreasing temperature [42]. In the ultra-low temperature 
range it behaves thermally as a dielectric. The application of an external magnetic field higher than $B_{c}$ forces it to be normal conducting. In the case with indium in the superconducting state there are four interfaces in the direction of the heat flow involving dielectric-like materials: copper - indium sc, indium sc - sapphire, sapphire - indium sc and indium sc - copper. The application of an external magnetic field reduces the amount of dielectric interfaces to two: indium nc - sapphire and sapphire - indium nc, and therefore improves the thermal performance of the sandwich. The measurement results of all three mentioned cases are described in detail in the following subsections.

\subsubsection{Thermal resistivity of the mechanically compressed setup}

In the first measurement campaign the setup was compressed with approx. $10 \mathrm{MPa}$ pressure, as described in chapter 3 .

\section{Indium in the normal conducting state}

The thermal resistivity of the sandwich with indium in the normal conducting state, for mixing chamber base temperatures of $30 \mathrm{mK}, 50 \mathrm{mK}$ and $70 \mathrm{mK}$, is presented in figure 6.1. The resistivity was calculated according to eq. (4.1). The thermal resistivity at different base temperatures plotted versus the stamp temperature overlap within the measurement precision. The error bars, increasing towards the low temperature end, represent the difficulty of a precise measurement of small temperature differences, as discussed in chapter 4. The slope of the lines would indicate that the behaviour of the setup depends not only on a pure interface resistance with a temperature dependence $R \propto T^{-3}$. Depending on the temperature, the thermal resistivity of the sandwich takes a value from $13 \mathrm{~cm}^{2} \mathrm{~K}^{4} / \mathrm{W}$ at the low temperature end to $27 \mathrm{~cm}^{2} \mathrm{~K}^{4} / \mathrm{W}$ at the high temperature end.

The theoretical value of the single sapphire - indium interface resistivity calculated according to eq. (5.7) for values of the speed of sound in the transverse direction, as summarised in table 2.1, equals to $23 \mathrm{~cm}^{2} \mathrm{~K}^{4} / \mathrm{W}$. This value should be multiplied by two to reflect the behaviour of a sandwich consisting of two interfaces in series: indium - sapphire and sapphire - indium. An average value of the resistivity in figure 6.1 is around $20 \mathrm{~cm}^{2} \mathrm{~K}^{4} / \mathrm{W}$. The fact that the sandwich has a resistivity close to the theoretical resistivity of a single interface, could be explained by the "parallel plates effect". At ultra-low temperatures the mean free path of phonons in a perfect crystal of sapphire reaches the value of the sapphire thickness. Phonons entering the cone of acceptance (according to AMM, section 2.4.1 on one side, almost don't scatter in the $1 \mathrm{~mm}$ thick sapphire disk, and they remain in the same cone at the second interface. In such situation, the two interfaces could be treated as one [4,64,77]. With an increase of temperature, the phonon mean free path shortens, phonons scatter more in 


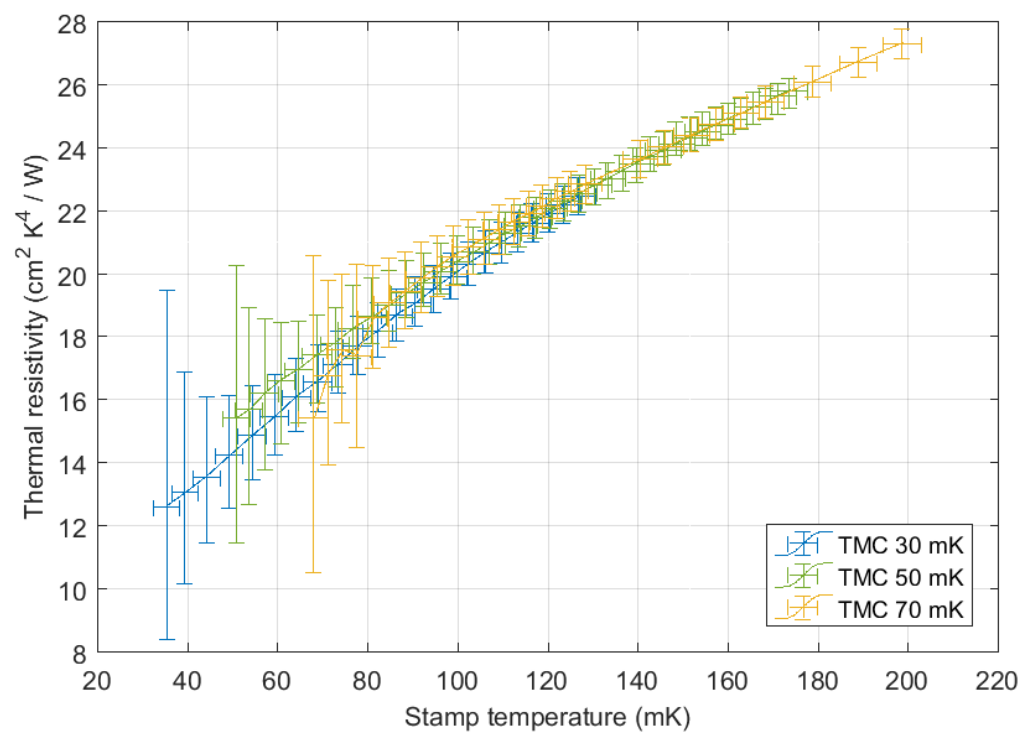

Figure 6.1: Thermal resistivity of the compressed setup with indium in the normal conducting state. Error bars representing the measurement uncertainty become larger towards the low temperature end, what represents the difficulty of measuring very small temperature differences, especially at very low temperatures.

the sapphire and the parallel plates effect becomes less pronounced. Following that assumption, the thermal resistivity should take a value of $23 \mathrm{~cm}^{2} \mathrm{~K}^{4} / \mathrm{W}$ at the lowest temperatures, and rise to $2 \cdot 23=46 \mathrm{~cm}^{2} \mathrm{~K}^{4} / \mathrm{W}$ with an increase of temperature. It could explain, why in figure 6.1 the value of resistivity rises with temperature, instead of being constant, as a perfect interface of Kapitzalike behaviour would be.

Another explanation of the non-zero slope of the thermal resistivity plot is the fact that the measured temperature gradients are definitely not small compared to the absolute temperature of the sandwich compounds. For high heat loads the temperature difference between the stamp and the platform is larger than the absolute temperature of the platform. For example, at a base MC temperature of $30 \mathrm{mK}$, when the stamp temperature reaches $120 \mathrm{mK}$, the platform is still at around $45 \mathrm{mK}$, so $\Delta T / T_{\mathrm{pl}}=(120-45) / 45=1.67$. Therefore measurements at ultra-low temperature may deviate from the perfect Kapitza theory, which predicts that the thermal resistivity calculated with eq. (2.21) and only for small temperature gradients the plot would be a flat line. The high temperature gradient and the power of 4 in equation (4.1) cause a linear increase of resistivity with temperature, which should be and is observed. In fact thermal boundary resistance measurements described in literature are almost never represented by a perfectly flat line and a certain slope is observed 4, 21, 25, 27, 51, 53, 77, 103, 104. 
The question remains why the thermal resistivity is lower than the theoretical value even for a single interface at the lowest measured temperatures. The low value of thermal resistivity at $30 \mathrm{mK}$ could be a result of the presence of the clamping structure, which compresses the setup with quite a high force. Perhaps at ultra-low temperatures, despite containing multiple layers of epoxy laminate and interfaces between them, the thermal resistivity of the clamping structure becomes comparable to the sandwich and there is some small, but significant amount of heat bypassing the sandwich. That influence will be evaluated later with the results of the decompressed setup and the setup without the clamping structure.

\section{Indium in the superconducting state}

Without the external magnetic field, indium is in the superconducting state which doubles the number of interfaces characterised by a dielectric-like behaviour, and significantly increases the total thermal resistivity of the setup. The significant interfaces are: copper - indium sc, indium sc - sapphire, sapphire - indium sc and indium sc - copper. The plot of the thermal resistivity of the compressed setup with indium in the superconducting state at various base temperatures, with comparison to the normal conducting state, is presented in figure 6.2. Large error bars below $150 \mathrm{mK}$ in the superconducting case are the

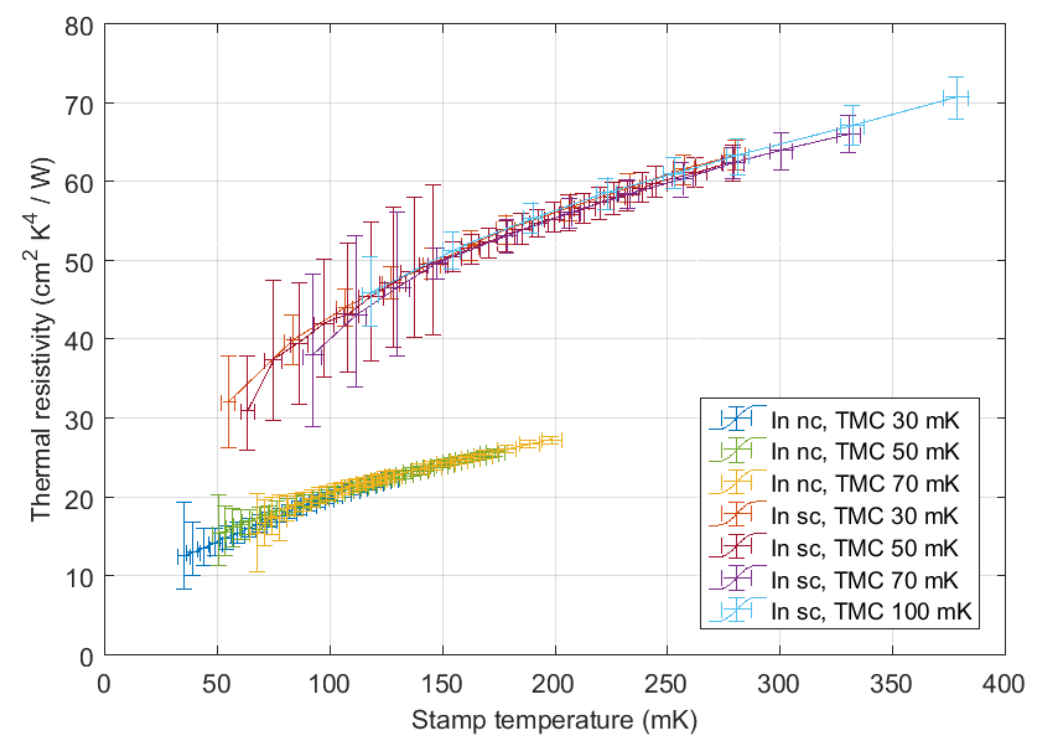

Figure 6.2: Thermal resistivity of the compressed setup with indium in the normal conducting and the superconducting states plotted as a function of the stamp temperature. Large error bars on the superconducting plot below $150 \mathrm{mK}$ result from an attempt to measure two different types of sensors in the same resistance range of the AVS bridge. 
result of an attempt to measure two sensors of very different electrical resistances in the same resistance range of the AVS bridge. The thermal resistivity of the setup is approximately two times higher than with indium in the normal conducting state, taking values from approximately 32 to $70 \mathrm{~cm}^{2} \mathrm{~K}^{4} / \mathrm{W}$. At stamp temperature of $100 \mathrm{mK}$ the thermal resistivity increases from 20 to $40 \mathrm{~cm}^{2} \mathrm{~K}^{4} / \mathrm{W}$, when the magnetic field is switched off. A theoretical estimation of the total sandwich resistivity, being a sum of four interfaces in series and taking the speed of sound for transverse phonons, results in a value of approx. $50 \mathrm{~cm}^{2} \mathrm{~K}^{4} / \mathrm{W}$. Including the parallel plates effect at the sapphire disk at the lowest temperatures, i.e. taking into account the following interfaces: copper - indium sc, indium sc - sapphire twice, but treated as one, and indium sc - copper, the total theoretical resistivity equals approximately to $27 \mathrm{~cm}^{2} \mathrm{~K}^{4} / \mathrm{W}$. The parallel plates assumption seems justified, as the value of $27 \mathrm{~cm}^{2} \mathrm{~K}^{4} / \mathrm{W}$ is close to the measured resistivity at the lowest temperatures $\left(32 \mathrm{~cm}^{2} \mathrm{~K}^{4} / \mathrm{W}\right)$. Towards higher temperatures the parallel plates assumption weakens, and therefore should lead to an increase of the thermal resistivity of a sandwich with an increase of temperature, and such a behaviour is observed.

Relatively large temperature gradients and some additional effects may also be present at the copper - indium sc interface that cause a deviation from a perfect Kapitza-like behaviour, which would be visualized by a flat line. Furthermore, it is relevant to consider the effects of the compression force and the clamping structure, and it will be described later.

\subsubsection{Thermal resistivity of the decompressed sandwich setup}

In the second measurement campaign the compression force was removed and the same setup was measured with the clamping structure still mounted, at base MC temperatures of $30 \mathrm{mK}, 50 \mathrm{mK}, 70 \mathrm{mK}$ and $100 \mathrm{mK}$ with indium in the normal conducting and the superconducting states.

\section{Indium in the normal conducting state}

A comparison of the normal conducting indium results with and without compression is interesting for the AEgIS project, where the space for the electrodes in the cold bore is limited. A possibility of eliminating the structure clamping the electrodes would be a big advantage, freeing space for instrumentation and thermal shields, and enabling a new electrode design. The $1 \mathrm{~T}$ magnetic field background around the ultra-cold trap in the AEgIS experiment guarantees that the indium remains in the normal conducting state. The comparison of results for the compressed and uncompressed sandwich setups is presented in figure 6.3. The curves representing the setup without compression lie a bit higher than curves representing the compressed setup, but the difference is comparable to the measurement precision. Respective error bars are not plot- 
ted for clarity, but are similar to the ones in figure 6.1. The plots overlap within the measurement accuracy, which means that the compression force does not have a significant influence on the thermal resistivity of the sandwich setup with indium in the normal conducting state.

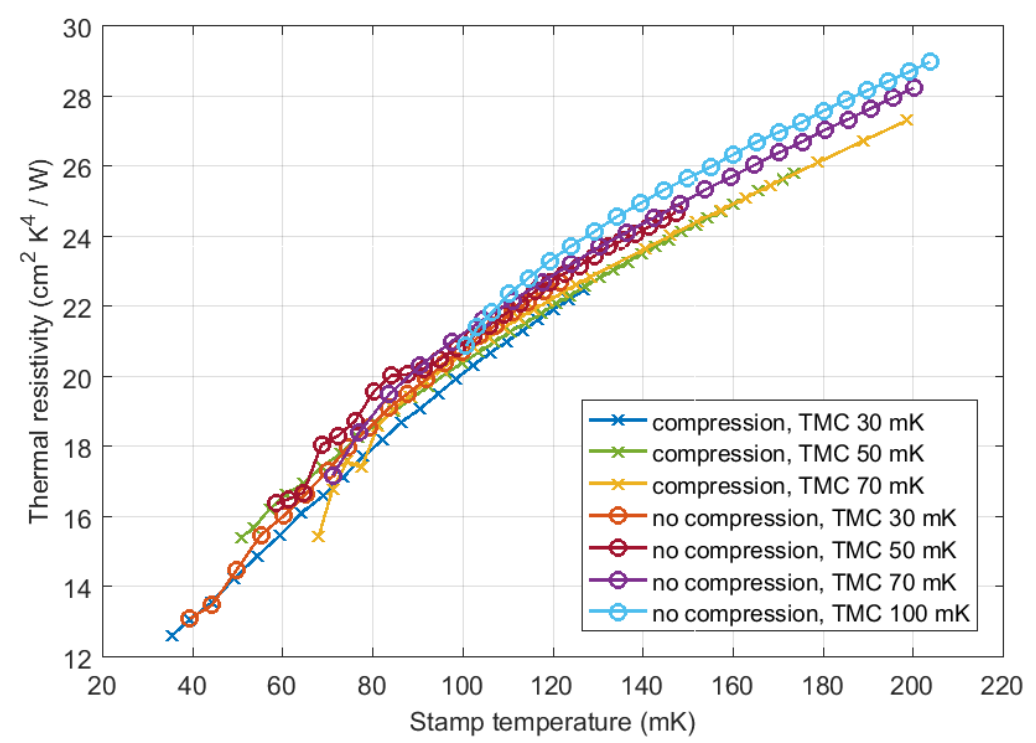

Figure 6.3: Comparison of the thermal resistivities of the compressed (x) and decompressed (o) setup, with normal conducting indium in both cases.

\section{Indium in the superconducting state}

The same comparison of the influence of the compression force is done for the case with superconducting indium. The thermal resistivities of the compressed and decompressed sandwich with indium in the superconducting state is plotted in figure 6.4. The presence of a compressing force has a significant influence on the results, decreasing the thermal resistivity. Unlike in the normal conducting case, the resistivity of the copper - indium interface is a significant element of the total resistivity. Results in figure 6.4 suggest that the indium - copper interface is less perfect than the sapphire - indium interface and therefore it is more sensitive to the presence of the compression force.

In the sandwich setup with indium in the normal conducting state, only the sapphire - indium interface involves a material with dielectric-like properties. i.e. sapphire. The surface of the sapphire was optically polished and indium was vapour deposited on it, which makes the interface quite "ideal" and its manufacturing procedure is reproducible. The surface of the sapphire disk was completely wetted by indium during the deposition providing a very good micro-scale contact. In the sandwich setup with indium in the supercon- 


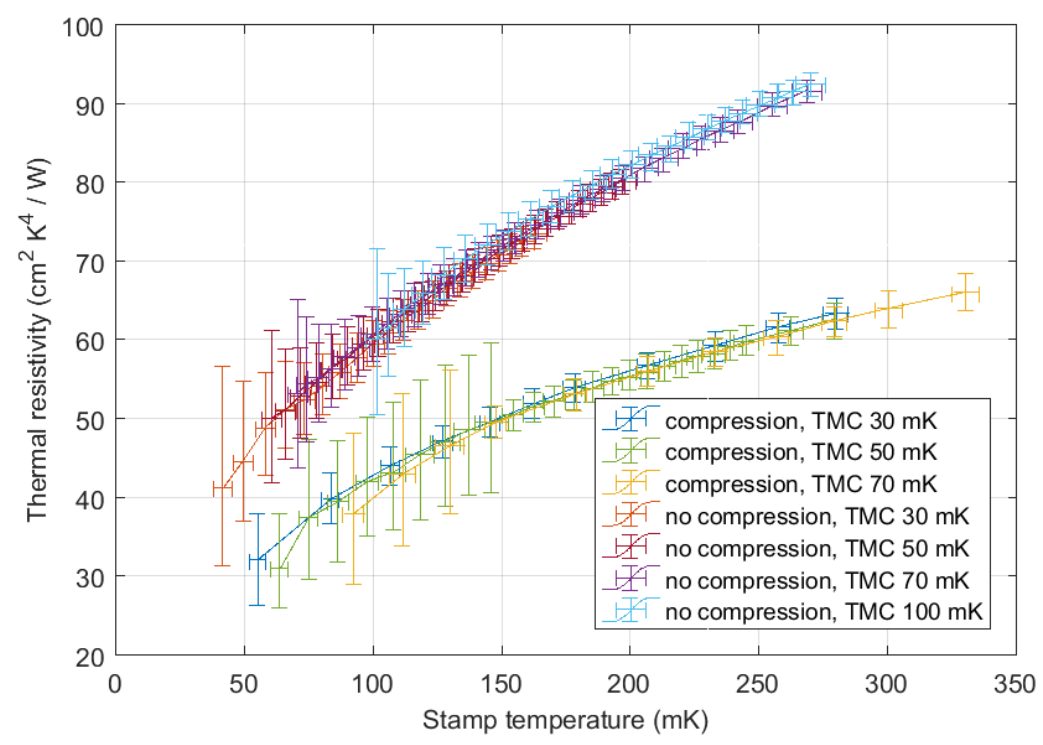

Figure 6.4: Comparison of the thermal resistivity for the compressed and not compressed setup, in both cases with superconducting indium.

ducting state, also the indium - copper interface is characterised by dielectric properties. However, the surface of copper is not perfectly polished and can have a trace of an oxide layer, what makes the thermal connection to indium less "perfect", and therefore more sensitive to the applied force. Moreover, with time and thermal cycles a certain ageing effect might appear, involving a formation of a new metallic interlayer between indium and copper. The ageing effect has already been noticed and described by other researchers [51]. An additional layer of an alloy having properties different than the two neighbouring metals could significantly increase the total thermal resistivity in the superconducting case. It could also explain why the measured thermal resistivity of the sandwich is much higher than the theoretical estimation, which equals to $27 \mathrm{~cm}^{2} \mathrm{~K}^{4} / \mathrm{W}$ (including the parallel plates assumption).

As described in section 6.1.1, the clamping structure might have bypassed part of the heat supplied to the stamp with the heater. The influence of the clamping structure is not significant for the normal conducting case (figure 6.2), because of a relatively low resistivity of the sandwich itself, which is the main heat path. However, in the superconducting case the resistance of the sandwich is several times higher and more comparable to the bypass resistance. Therefore the influence of the bypass is much more pronounced. Removal of the compression force from the clamping structure should increase its thermal resistance even more than the resistance of the sandwich. Much less heat flows through the decompressed clamping structure, and the calculated thermal resistivity of 
the setup is higher.

The fact that lines representing the decompressed setup lie much higher than the compressed setup can thus be a result of two effects: ageing of the copper - indium interface, or the bypass that might have falsified the results for the compressed setup. A measurement of the setup without the clamping structure present at all could help to distinguish which of these effects is dominant. These results are present in the following section.

\subsubsection{Sandwich without the clamping structure}

The clamping structure was designed in a way to minimize the potential heat flow that could bypass the sandwich, flowing from the stamp to the platform via the clamping structure. However, despite 20 layers of G10 and several elements made of stainless steel, which because of its grainy alloy structure has a relatively low thermal conductivity at low temperatures (see figure 2.4), the amount of heat flowing via the clamping remains uncertain. Judging by the results presented in section 6.1.1 and 6.1.2 that amount of heat may not be negligible. Two more measurement runs were done with the clamping structure completely removed. The first in a $\mathrm{MC}$ temperature range from $30 \mathrm{mK}$ to $100 \mathrm{mK}$, and the second in a much wider $\mathrm{MC}$ temperature range from $30 \mathrm{mK}$ to $300 \mathrm{mK}$.

\section{Indium in normal conducting state}

Results of the measurements without the clamping structure compared to the previous measurements of the compressed and the decompressed setup, and to the results obtained by T. Eisel [4], always with indium in the normal conducting state, are presented in figure 6.5.

The curves corresponding to the two runs without the clamping structure are close to overlap within the measurement precision and they seem to have a constant offset comparing to the results from the two measurement runs with the clamping structure mounted on the sandwich (compressed, decompressed). It indicates that the presence of the clamping structure changed the path of the heat flow, as the thermal resistivity of the setup without clamping structure is higher than with the clamping structure. The clamping structure in the DR is built mainly of G10 layers, which is an epoxy - glass fibre composite. Peterson and Anderson [77], who conducted low temperature measurements of various interface resistances, wrote that the thermal conductivity of epoxy has a temperature dependence of $\lambda_{\text {epoxy }} \propto T^{2}$. Thus, the corresponding amount of bypassing heat could be estimated as:

$$
\dot{Q}_{\text {bypass }}=3 \gamma_{\text {bypass }} \int_{T_{\text {platform }}}^{T_{\text {stamp }}} T^{2} d T=\gamma_{\text {bypass }}\left(T_{\text {stamp }}^{3}-T_{\text {platform }}^{3}\right),
$$




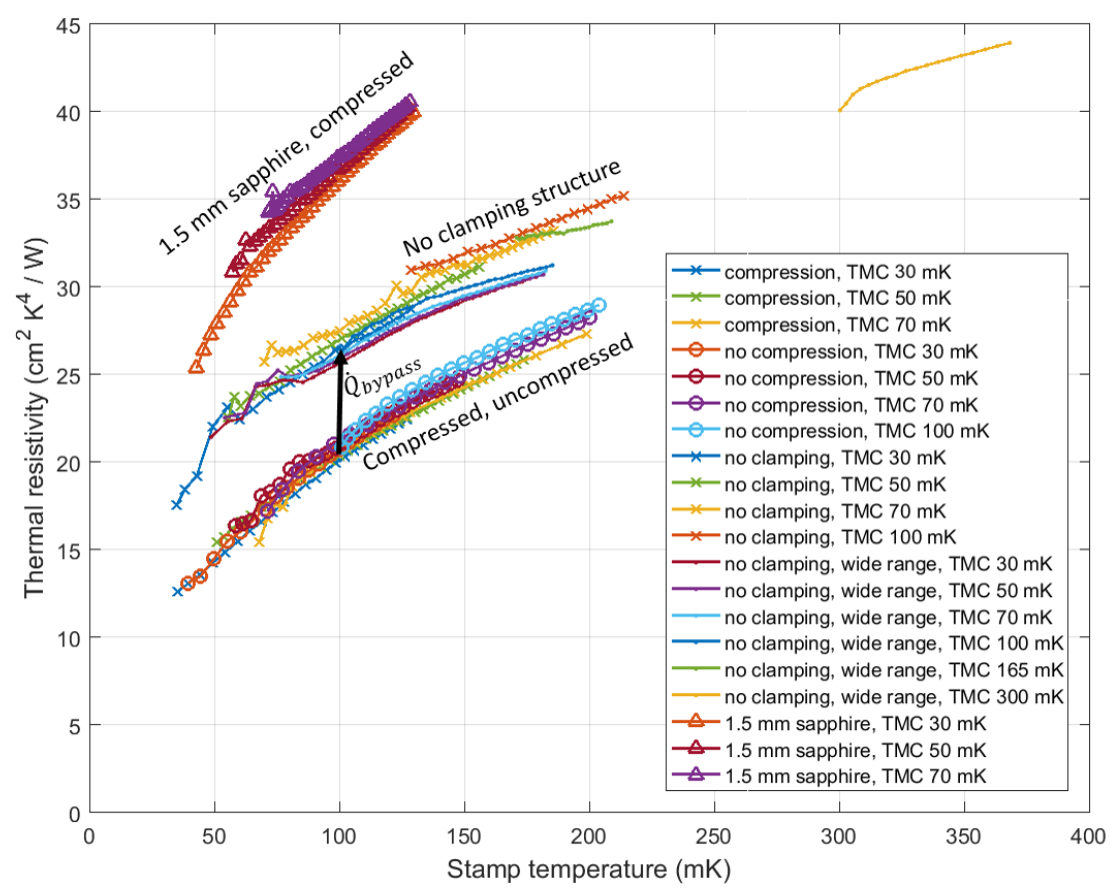

Figure 6.5: Thermal resistivity results with normal conducting indium for the cases: compressed, decompressed, without clamping structure, without clamping structure in a wide temperature range, and compared with results obtained by T. Eisel for $1.5 \mathrm{~mm}$ thick sapphire disk, compressed with the same clamping structure 4 . Including a parasitic heat flow via the clamping structure $\dot{Q}_{\text {bypass }}=\gamma_{\text {bypass }}\left(T_{\text {stamp }}^{3}-T_{\text {platform }}^{3}\right)$ would shift the results for the compressed and the decompressed setup exactly to the position of the curves representing the setup without the clamping structure.

where $\gamma$ is a constant. It can be demonstrated that if the amount of heat flowing via the clamping structure was proportional to $T_{\text {stamp }}^{3}-T_{\text {platform }}^{3}$ with the proportionality coefficient $\gamma \approx 0.001 \mathrm{~W} / \mathrm{K}^{3}$, the results for both the compressed and decompressed setup would be shifted towards higher resistivities and almost exactly overlap with the results for the setup without the clamping structure. The temperature dependence $\dot{Q}_{\text {bypass }}=\gamma\left(T_{\text {stamp }}^{3}-T_{\text {platform }}^{3}\right)$ may also reflect a combination of different contributions to the total resistivity of the clamping structure: an interface resistivity between multiple layers $\left(R \propto T^{-3}\right)$, a bulk dielectric resistivity of G10 $\left(R \propto T^{-2}\right)$, and a bulk metallic resistivity of brass and stainless steel $\left(R \propto T^{-1}\right)$.

T. Eisel measured the same setup with a $1.5 \mathrm{~mm}$ thick sapphire disk and compressed with a similar force as the first described case. Thus his results should be compared mainly to the compressed setup case. The increase of the sapphire thickness by $50 \%$ increases the thermal resistivity of the sandwich 
almost twice, e.g at $50 \mathrm{mK}$ the resistivity rises from $15 \mathrm{~cm}^{2} \mathrm{~K}^{4} / \mathrm{W}$ to almost $30 \mathrm{~cm}^{2} \mathrm{~K}^{4} / \mathrm{W}$, when the thickness of the sapphire disk changes from $1 \mathrm{~mm}$ to $1.5 \mathrm{~mm}$. The thicker the sapphire disk, the higher the chance that the phonons get scattered, and the less justified the parallel-plates assumption. It may also be, that the quality of the copper - indium - sapphire connection differs slightly between the setup of T. Eisel and the setup presented in this thesis, despite the same mounting procedure. Many authors mention irreproducibility of the interface results [53,77].

The influence of the clamping structure on the thermal resistivity of the sandwich is thus much lower, than the cumulative effect of the $50 \%$ change of the sapphire thickness and the re-assembly of the setup.

When indium is in the normal conducting state and the electrons are available for the heat transport, the ageing and alloying effect remains invisible, and the thermal resistivity does not change significantly with time or with thermal cycles (figure 6.5), what is an important information for the application in AEgIS. Furthermore, the results show that for the new electrode the clamping structure can be removed once the indium bond has been established.

\section{Indium in the superconducting state}

The thermal resistivities of the setup with indium in the superconducting state in the same configurations: mechanically compressed, decompressed, without clamping structure, and without clamping structure in a wide temperature range is presented in figure 6.6. The thermal resistivity of the first two cases (the compressed and the decompressed sandwich) do not overlap as already discussed in section 6.1.2. The thermal resistivities obtained in the two measurement runs without the clamping structure are very close to each other, which is supporting the results. They take a value from $100 \mathrm{~cm}^{2} \mathrm{~K}^{4} / \mathrm{W}$ to $163 \mathrm{~cm}^{2} \mathrm{~K}^{4} / \mathrm{W}$. The suspicion, that the compressing force was not fully released in the decompressed setup run, seems justified, as the results for the decompressed setup are closer to the compressed one than to the setup without the clamping structure. However, the remaining compression force should not be very high. It is difficult to explain the large increase of the thermal resistivity between the decompressed setup and the two runs without the clamping structure, only with an elimination of the clamping. There should be additional explanations.

The ageing or alloying process at the "imperfect" copper - indium sc interface might have also caused an increase of the resistivity from run to run. It is probable that the alloying process was more intense at the beginning in the first run, when the compression force was applied (and a partial compression in the second run). Accordingly, the influence of that effect should be more pronounced between the first two runs, than the last two runs. A trace of that effect may be still visible between the results obtained in the $3^{\text {rd }}$ run 


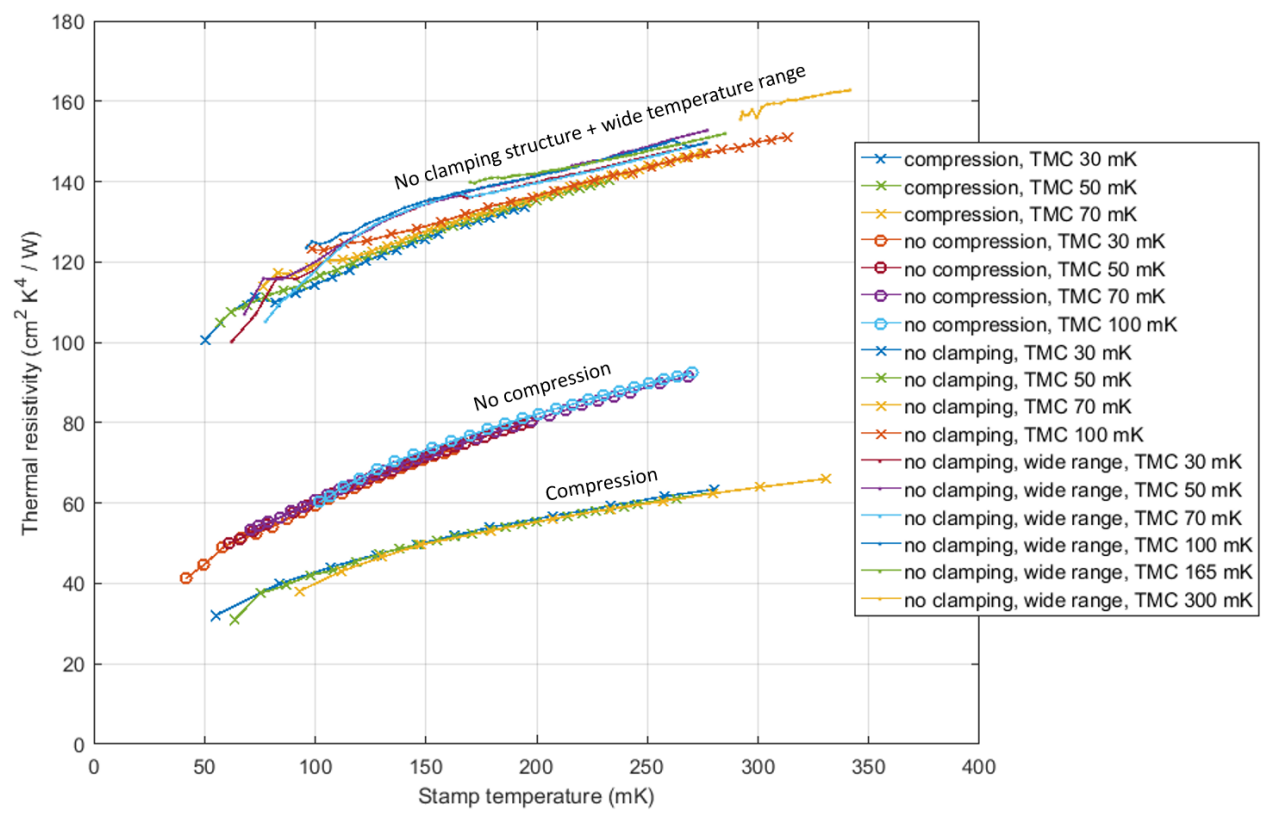

Figure 6.6: Thermal resistivity of the compressed setup, decompressed, without clamping structure, and without clamping structure in a wide temperature range, versus the temperature of the stamp, with indium in the superconducting state.

- without the clamping structure, and in the $4^{\text {th }}$ measurement run - in a wide temperature range. The resistivity in the run without the clamping structure is a bit lower than the resistivity from the run in an extended temperature range (figure 6.6), but the difference is comparable with the measurement error.

The behaviour of the copper - indium sc interface is totally different than the sapphire - indium nc interface, due to electron availability for heat transfer in normal conducting indium. As concluded from the results with indium in the normal conducting state, the quality of the sapphire - indium connection and the validity of the parallel-plates assumption should not change with time or thermal cycles.

The $125 \mu \mathrm{m}$ layer of indium was compressed above its elastic limit during the mounting, to make sure the cold weld is obtained (see chapter 3). The grainy, stressed layer of indium itself contains a lot of dislocations and probably some traces of oxidation, so it is not expected to have a very high thermal conductivity in the superconducting state, when the electrons are not available for heat transfer. The alloying effect at an interface with copper could make its conductivity even lower.

Measurements in almost all of the runs were done first without the external magnetic filed, i.e. with indium in the superconducting state, and afterwards 
with magnetic field, to make sure that no magnetic flux is trapped in the superconductor. Trapped flux would prevent indium (and titanium) from obtaining its fully superconducting properties. The decompressed setup was the only one measured first with an external magnetic field and later without it. Therefore, there might have been some flux trapped in indium and some electrons might have been available for heat transport. An electronic contribution to the heat transfer would decrease the effective thermal resistivity of the sandwich and could explain the steeper slope of the lines representing the decompressed setup in figure 6.6 ,

To sum up, the quality of the indium - copper interface, the ageing effect, and the layer of superconducting indium have a very big influence on the thermal resistivity of the sandwich. The values measured in the runs without the clamping structure are way above the theoretical estimation of $27 \mathrm{~cm}^{2} \mathrm{~K}^{4} / \mathrm{W}$ (estimation for the speed of sound in the transverse direction, including the parallel plates assumption), or even $50 \mathrm{~cm}^{2} \mathrm{~K}^{4} / \mathrm{W}$ (estimation for the speed of sound in the transverse direction, without the parallel plates assumption included).

\subsubsection{Comparison with the model}

In the mathematical model explained in chapter 5 the stamp temperatures have been estimated based on the platform temperatures and the theoretical prediction of the interface resistivities. Here, the interface resistivities $\kappa$ and characteristic conductivities of thin layers $\alpha$ will be calculated using the same electric analogies, equations (5.4), (5.8) and the measured temperatures. All the calculations are based on the last measured configuration - without clamping structure in a wide temperature range, since that is the configuration that seems the most attractive for AEgIS.

\section{$\mathrm{Cu}-$ In sc - Cu connection}

In section 5.1 a chain of three resistances in series, representing two $\mathrm{Cu}$ - In interfaces and the indium layer in between, is described. For each measurement point of a defined heat load and known stamp and platform temperatures, three heat transfer equations corresponding to these resistors can be written, as eq. (5.4) and eq. (5.8). They contain two unknown temperatures, marked as $T_{2}$ and $T_{3}$ in figure 5.1, and four uncertain values: $\kappa_{\mathrm{Cu}-\mathrm{In}}, n_{\mathrm{Cu}-\mathrm{In}}, \alpha_{\mathrm{In} \mathrm{sc}}, n_{\mathrm{In} \mathrm{sc}}$. Assuming that the temperature dependence of all three resistances follows the theoretical prediction $R \propto T^{-3}$, i.e. $n_{\mathrm{Cu}-\mathrm{In}}=3$ and $n_{\mathrm{In} \mathrm{sc}}=3$, and treating $\kappa_{\mathrm{Cu}-\mathrm{In}}$ and $\alpha_{\mathrm{In} \text { sc }}$ as unknowns which should not change with the heat load, it is possible to solve a system of 6 equations ( 3 equations for two consecutive measurement points) with 6 unknowns: 4 intermediate temperatures, $\kappa_{\mathrm{Cu}-\mathrm{In}}$ and $\alpha_{\mathrm{In} \mathrm{sc}}$. 
The obtained solution shows that the resistance corresponding to the $125 \mu \mathrm{m}$ layer of indium is way lower than the interface resistance and equals $\kappa_{\mathrm{In} \text { sc }}=$ $l_{\text {In }} / \alpha_{\text {In sc }}=\frac{125 \mu \mathrm{m}}{0.0888 \mathrm{~W} /\left(\mathrm{cm} \mathrm{K}^{4}\right)}=\frac{1}{7.09 \mathrm{~W} /\left(\mathrm{cm}^{2} \mathrm{~K}^{4}\right)}=0.141 \mathrm{~cm}^{2} \mathrm{~K}^{4} / \mathrm{W}$. The calculated interface resistivity $\kappa_{\mathrm{Cu}-\mathrm{In}}$ plotted as a function of the upper interface temperature is presented in figure 6.7 .

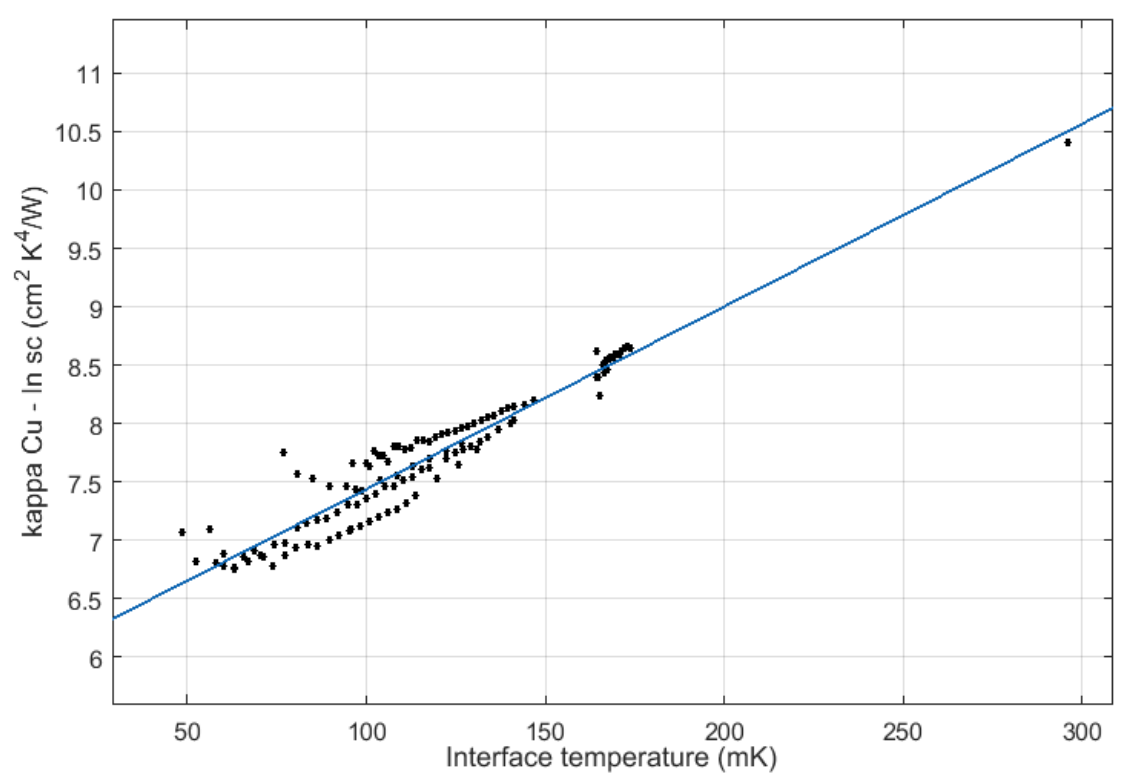

Figure 6.7: Calculated values of $\mathrm{Cu}$ - In interface resistivity as a function of the calculated upper (warmer) interface temperature. The blue line is a linear fit with a formula $\kappa_{\mathrm{Cu}-\mathrm{In}}=$ $15.65 T+5.87$ (for $T$ in $\mathrm{K}$ ).

If the measured temperature gradients were really small, the total resistance could be approximated by $R=\kappa T^{-3}$, with $\kappa$ being a constant. For significant temperature gradients it is necessary to include in the model the first - linear term of the Taylor expansion, therefore the blue line is a fit with a formula $a T+b$, which seems to correctly represent the fitted data, for $a=15.65$ and $b=5.87$ for $T$ in $\mathrm{K}$. All the calculated resistivity values are summarised in table 6.1. Other researchers also observed a linear temperature depedence of $\kappa$ [4, 53, 77]. It may be that a difficult to quantify oxidation of the surface or another unpredicted effect also contributes to the temperature dependence $\frac{d \kappa}{d T}=15.65$. The interface resistivity $\kappa_{\mathrm{Cu}-\mathrm{In}}$ calculated here will be used in more complex configurations of the setup to calculate resistivities of other interfaces.

\section{$\mathrm{Cu}$ - In nc - sapphire}

The same approach was used to calculate the sapphire - indium interface resistivity from the model described in section 5.2 . The temperature dependence 
Table 6.1: The theoretical and calculated values of the characteristic interface resistivities and thin layer conductivities present in the $\mathrm{Cu}-\mathrm{In}-\mathrm{Cu}$ setup. The values in the right column are calculated from the model based on the experimental data.

\begin{tabular}{|c|c|c|}
\hline Value & Theoretical resistivity & Calculated from the model \\
\hline$\kappa_{\text {In sc }}=l_{\text {In }} / \alpha_{\text {In sc }}$ & $0.141 \mathrm{~cm}^{2} \mathrm{~K}^{4} / \mathrm{W}$ & $0.141 \mathrm{~cm}^{2} \mathrm{~K}^{4} / \mathrm{W}$ \\
$\kappa_{\text {In }-\mathrm{Cu}}$ & $2.09 \mathrm{~cm}^{2} \mathrm{~K}^{4} / \mathrm{W}$ & $15.65 T+5.87 \mathrm{~cm}^{2} \mathrm{~K}^{4} / \mathrm{W}$ \\
\hline
\end{tabular}

of the sapphire - indium interface resistance is assumed to be equal to the theoretical prediction $R=\kappa_{\text {sapphire-indium }} T^{-3}$ and the temperature dependence of a sapphire thermal conductivity is modelled as $\lambda_{\text {sapphire }}=\alpha_{\text {sapphire }} T^{3}$. The values of $\kappa_{\text {sapphire-indium }}$ and $\alpha_{\text {sapphire }}$ were obtained from the measured data by solving the system of 6 equations for every pair of two neighbouring measurement points (two different heat loads). The calculated thermal conductivity of sapphire compared to literature data and the values of the interface resistivities are summed up in table 6.2

Table 6.2: The theoretical and calculated values of the characteristic interface resistivities and thin layer conductivities present in the $\mathrm{Cu}$ - In nc - sapphire setup.

\begin{tabular}{|c|c|c|}
\hline Value & Theoretical & Calculated from the model \\
\hline$\kappa_{\text {sapphire-In }}^{\text {Single intere }}$ & $22.96 \mathrm{~cm}^{2} \mathrm{~K}^{4} / \mathrm{W}$ & $35.46 T+10.17 \mathrm{~cm}^{2} \mathrm{~K}^{4} / \mathrm{W}$ \\
$\alpha_{\text {sapphire }}$ & $0.023 \mathrm{~W} /\left(\mathrm{cm} \mathrm{K}^{4}\right)$ & $167 \mathrm{~W} /\left(\mathrm{cm} \mathrm{K}^{4}\right)$ \\
$\kappa_{\text {sapphire }}=l_{\text {sapph }} / \alpha_{\text {sapph }}$ & $0.043 \mathrm{~cm}^{2} \mathrm{~K}^{4} / \mathrm{W}$ & $6 \cdot 10^{-4} \mathrm{~cm}^{2} \mathrm{~K}^{4} / \mathrm{W}$ \\
$\kappa_{\text {sapphire dist }}^{\text {paralles }}$ & $22.96 \mathrm{~cm}^{2} \mathrm{~K}^{4} / \mathrm{W}$ & $67.97 T+21.29 \mathrm{~cm}^{2} \mathrm{~K}^{4} / \mathrm{W}$ \\
\hline
\end{tabular}

The calculated resistance corresponding to the sapphire disk is very low compared to the resistances of the interfaces, and the estimation of the phonon mean free path in sapphire below $1 \mathrm{~K}$ gives a value of approx. $2 \mathrm{~mm}$. Therefore, it seems to be a correct approach to model the two interfaces surrounding the sapphire disk according to the parallel plates assumption described in section 5.2. After including the parallel plates assumption in the model, the calculated sapphire - In interface resistivity, which is rising with temperature, was fitted linearly obtaining: $\kappa_{\text {sapphire disk }}^{\text {parallel }}=67.97 T+21.29 \mathrm{~cm}^{2} \mathrm{~K}^{4} / \mathrm{W}$, as presented in figure 6.8. The temperature dependence $\frac{d \kappa}{d T}=67.97$ can be explained with the fact that the parallel plates assumption should become less valid with the temperature rise, when phonons start scattering more and more in the sapphire disk. The $\kappa_{\text {sapphire disk }}^{\text {parallel }}$ found here will be used in models of more complex configurations of the setup.

\section{$\mathrm{Cu}$ - In sc - sapphire}

It is assumed that the sapphire - indium interface does not change its properties depending on the presence of the external magnetic field, and that the 


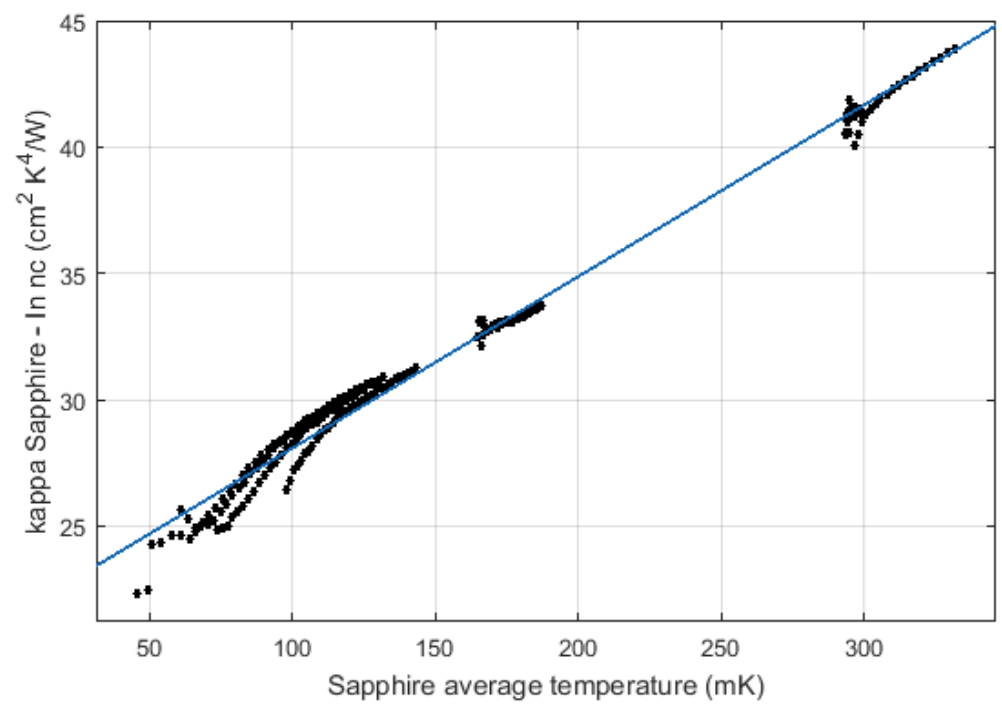

Figure 6.8: Calculated values of In - sapphire - In resistivity including the parallel plates assumption as a function of the calculated sapphire temperature. The blue line is a linear fit with a formula $\kappa_{\text {sapphire disk }}^{\text {parallel }}=67.97 T+21.29 \mathrm{~cm}^{2} \mathrm{~K}^{4} / \mathrm{W}$ (for $T$ in $\mathrm{K}$ ).

properties and thickness of the layer of indium foil are the same as in the lid platform connection. In the model of the copper - indium sc - sapphire configuration $\kappa_{\text {sapphire disk }}^{\text {parallel }}$ and $\alpha_{\text {In sc }}$ calculated above will be used to find the new value of the $\kappa_{\mathrm{Cu}-\mathrm{In} \text { sc }}$ and the exponent describing the temperature dependence $n$ defined as $R=\kappa T^{-n}$. As explained before the $\mathrm{Cu}$ - In interface resistance depends heavily on the amount of oxidation and on the intermetallic alloys formed and therefore can vary significantly between the measured cases. The exponent $n$ of the resistance temperature dependence is found to be $n_{\mathrm{Cu}-\mathrm{In} \mathrm{sc}}=2.88<3$, what shows that the alloyed interface differs slightly from an ideal AMM interface, having more electronic contribution to the heat transfer. The copper indium interface resistivity for the sandwich case calculated for $n=3$ and fitted linearly as a function of temperature gives: $\kappa_{\mathrm{Cu}-\mathrm{In} \mathrm{sc}}^{\text {sandwich }}=53.75 T+43.61$. The temperature dependence of $\kappa_{\mathrm{Cu}-\mathrm{In} \text { sc }}^{\mathrm{sandwich}}$ is stronger than for $\kappa_{\mathrm{Cu}-\mathrm{In} \text { sc }}$ calculated from the lid to platform connection (figure 6.7). It agrees with the assumption that the resistivity of that type of connection can vary significantly. The higher resistivity may be a result of the fact that the sandwich was compressed with a lower force than the lid to platform connection, and therefore the formed bond has different properties. The theoretical AMM resistivity values and the values obtained from the model for this configuration of the setup are summed up in table 6.3. 
Table 6.3: The theoretical and calculated values of the characteristic resistance parameters in the $\mathrm{Cu}$ - In sc - sapphire setup.

\begin{tabular}{|c|c|c|}
\hline Value & Theoretical & Calculated from the model \\
\hline$\kappa_{\text {sapphire disk }}^{\text {parallel plates }}$ & $22.96 \mathrm{~cm}^{2} \mathrm{~K}^{4} / \mathrm{W}$ & $67.97 T+21.29 \mathrm{~cm}^{2} \mathrm{~K}^{4} / \mathrm{W}$ \\
$\kappa_{\text {Cu-In sc }}^{\text {sandwich }}$ & $2.09 \mathrm{~cm}^{2} \mathrm{~K}^{4} / \mathrm{W}$ & $53.75 T+43.61 \mathrm{~cm}^{2} \mathrm{~K}^{\mathrm{n}} / \mathrm{W}$ \\
$n_{\mathrm{Cu}-\mathrm{In} \text { sc }}^{\text {sandwich } R=\kappa T^{-n}}$ & 3 & 2.88 \\
\hline
\end{tabular}

The findings from the analysis of the $\mathrm{Cu}$ - In - sapphire sandwich will be applied to the analysis of the measurement results of the second sandwich setup, that is discussed in section 6.2 .3 .

\subsection{Steady state measurements of the copper - indium - gold - titanium - sapphire sandwich setup}

The second and more complicated sandwich consists of the following layers (fig. 5.14 :

- stamp made of OFHC copper,

- $125 \mu \mathrm{m}$ thick indium foil,

- $520 \mathrm{~nm}$ gold (as measured and described in section 3.1.3),

- $45 \mathrm{~nm}$ of titanium (as measured and described in section 3.1.3),

- $1 \mathrm{~mm}$ thick polished sapphire disk,

- $45 \mathrm{~nm}$ of titanium,

- $520 \mathrm{~nm}$ of gold,

- $125 \mu \mathrm{m}$ thick indium foil,

- platform made of OFHC copper,

The thin layers of titanium and gold were deposited on a polished sapphire with a magnetron sputtering technique. The setup was compressed at room temperature and the clamping structure was removed before the measurements. It was measured in a wide temperature range, for the case without an external magnetic field even up to the stamp temperature of $500 \mathrm{mK}$. 


\subsubsection{Measurements with an external magnetic field}

By applying an external magnetic field the number of significant interfaces, i.e. including dielectric-like properties, reduces to two: titanium - sapphire and sapphire - titanium. The plot of the thermal resistivity of the sandwich with indium and titanium in the normal conducting state and with error bars indicating the accuracy of the measurement is presented in figure 6.9. As in previous measurements, the size of the error bars increases when the temperature decreases due to the small heat loads applied on the stamp, what visualises the difficulty of measuring very small temperature differences. The curves representing the results at various base temperatures overlap within the measurement precision.

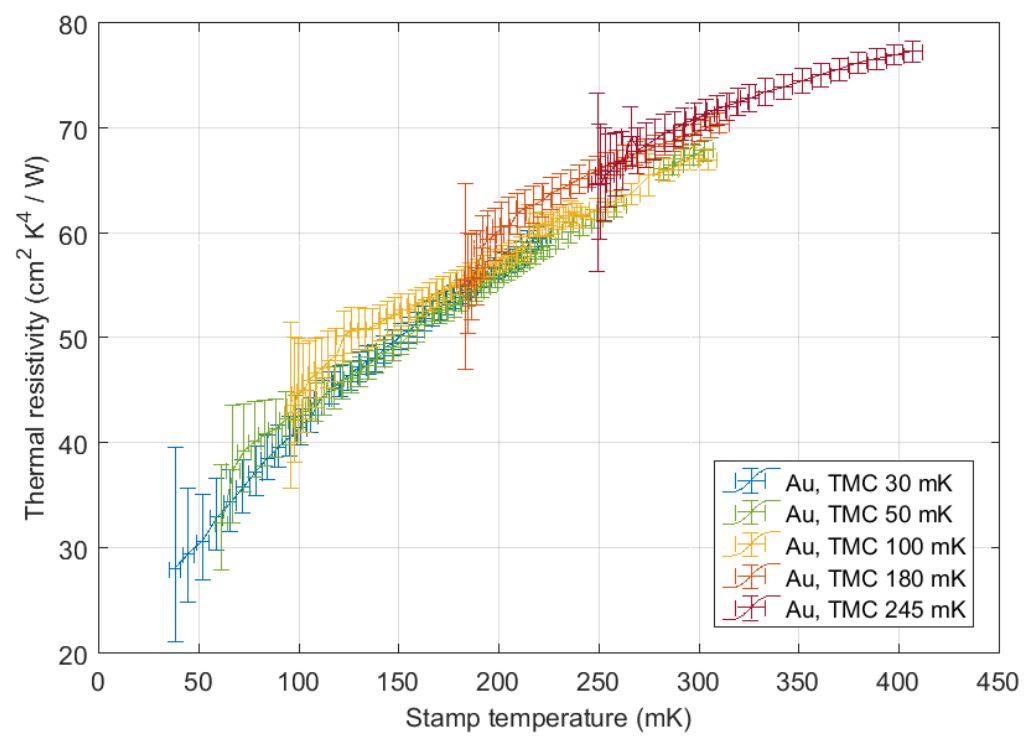

Figure 6.9: The thermal resistivity of the copper - indium - gold - titanium - sapphire sandwich, with indium and titanium in the normal conducting state as a function of the stamp temperature with varying base temperature of the mixing chamber.

The measured thermal resistivity of the sandwich with titanium and indium in the normal conducting state takes a value from approx. $28 \mathrm{~cm}^{2} \mathrm{~K}^{4} / \mathrm{W}$ at the low temperature end to $78 \mathrm{~cm}^{2} \mathrm{~K}^{4} / \mathrm{W}$ at the high temperature end. The theoretical estimation of the interface resistance, including the parallel plates assumption, i.e. treating the two interfaces titanium - sapphire and sapphire titanium as one, gives a value of about $18.3 \mathrm{~cm}^{2} \mathrm{~K}^{4} / \mathrm{W}$. This estimation predicts a lower resistivity than the measured one, even at the lowest temperatures. The mechanism of adhesion of titanium to sapphire most probably involves a formation of an ultra-thin layer of $\mathrm{TiO}$. Such an oxide layer may significantly increase the sapphire - titanium interface resistivity, or perhaps even deteriorate the parallel plates effect by scattering phonons at the interface. With an 
increase of temperature the parallel plates assumption becomes even less valid, and at the high temperature end, when the two interfaces should be treated completely separately, the thermal resistivity would take a theoretical value of about $36.6 \mathrm{~cm}^{2} \mathrm{~K}^{4} / \mathrm{W}$. The measured values are roughly a factor of two higher than the theoretical prediction.

A comparison of the thermal resistivities of the first sandwich setup with the second setup with magnetic field applied is presented in figure 6.10. The thermal resistivity of the second sandwich is approximately a factor of two higher. Dissimilarity between the acoustic impedances of sapphire and indium is bigger than between sapphire and titanium, so according to the AMM (section 2.4.1), the thermal resistivity of the second sandwich with titanium nc and gold should be lower than of the setup with only indium, assuming that the resistivity of all metallic - metallic interfaces is negligible compared to dielectric metallic interfaces. The fact, that the resistivity of the sandwich with titanium nc and gold is higher than the copper - indium nc - sapphire setup, indicates that another source of thermal resistance exists in the $\mathrm{Ti}$ - $\mathrm{Au}$ sandwich, even when titanium and indium are in the normal conducting state.

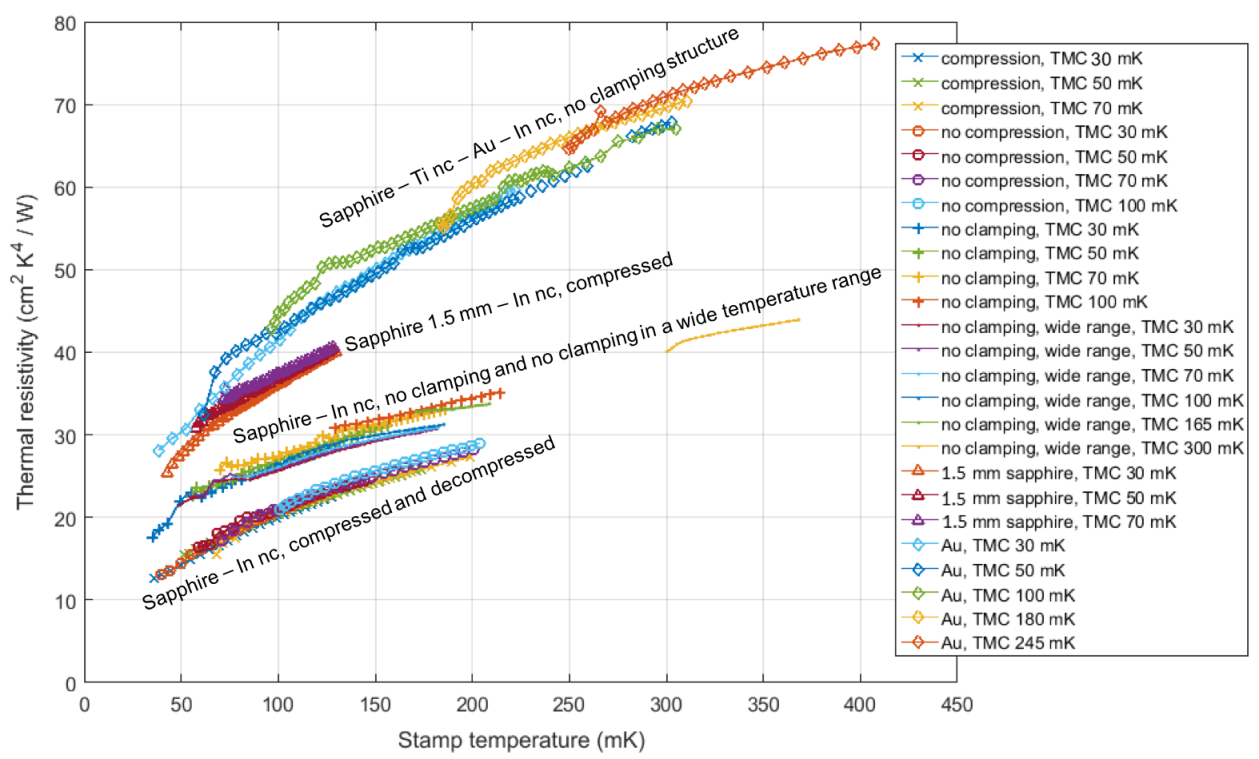

Figure 6.10: A comparison of the thermal resistivities of the two sandwich setups in all measured configurations with magnetic field applied.

The STEM imagining described in section 3.1.3 showed that both titanium and gold have a polycrystalline, grainy structure. A polycrystalline structure of thin films of gold significantly reduces its effective thermal conductivity 33. The thermal conductivity of thin layers has been discussed in section 2.5. Many examples show that the conductivity of thin layers of both metals and 
dielectrics decreases several times, when the thickness of the layer is smaller than the mean free path of the heat carriers. Since at ultra-low temperatures the wavelength associated with heat carriers in thin layers of gold and titanium is longer than the thicknesses of these layers (see figure 5.12), the observed $2 \mathrm{x}$ reduction of the thermal conductivity compared to the theoretical value is very well possible, even when the layers are in the normal conducting state.

\subsubsection{Measurements without an external magnetic field}

Titanium becomes superconducting below $0.39 \mathrm{~K}$ [31]. Thanks to that, without the application of an external magnetic field, it is possible to separately analyse the influence of the superconducting thin layers of titanium and indium. When titanium switches from the superconducting to the normal conducting state at $0.39 \mathrm{~K}$, the observed change of resistivity corresponds to the thermal resistivity caused mainly by the dielectric-like thermal properties of the superconducting thin layer of titanium. A plot of the thermal resistivity of the second sandwich in the temperature range of the stamp from $60 \mathrm{mK}$ to $500 \mathrm{mK}$ is presented in figure 6.11.

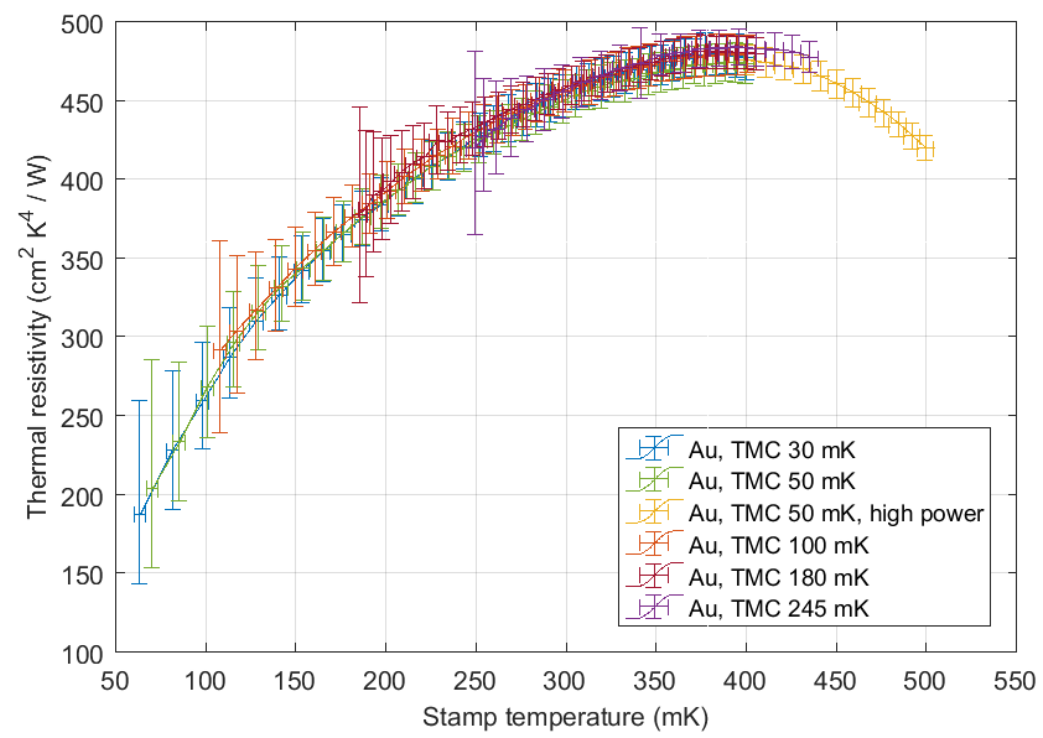

Figure 6.11: The thermal resistivity of the copper - indium - gold - titanium - sapphire sandwich setup, with indium in the superconducting state and titanium changing from the superconducting to the normal conducting state at its critical temperature of $0.39 \mathrm{~K}$.

The thermal resistivity takes a value of approximately $180 \mathrm{~cm}^{2} \mathrm{~K}^{4} / \mathrm{W}$ at the lowest measured stamp temperature of $60 \mathrm{mK}$. The highest thermal resistivity value of about $480 \mathrm{~cm}^{2} \mathrm{~K}^{4} / \mathrm{W}$ was measured for a stamp temperature of $396 \mathrm{mK}$ and at base MC temperature of $245 \mathrm{mK}$, just before titanium switches to the 
normal conducting state. The elimination of the dielectric behaviour of the thin layer of titanium due to the change from sc to nc state starts to significantly reduce the thermal resistivity of the sandwich above $0.4 \mathrm{~K}$ stamp temperature.

The sandwich with thin layers of titanium, gold and indium, when indium and titanium are superconducting, contains twice the number of interfaces involving a dielectric-like behaviour than the first setup with indium in the superconducting state:

1. copper - indium sc,

2. indium sc - gold,

3. gold - titanium sc,

4. titanium sc - sapphire.

All of these interfaces are repeated symmetrically on the other side of the sapphire, what gives in total 8 interfaces. The theoretical estimation of the sum of the interface resistivities including the parallel-plates assumption at the sapphire disk results in approximately $27 \mathrm{~cm}^{2} \mathrm{~K}^{4} / \mathrm{W}$ (for the speed of sound of transverse phonons). The estimated value is 6 times lower than the measured thermal resistivity at the lowest temperature. Without including the parallelplates assumption, the estimation gives approx. $45 \mathrm{~cm}^{2} \mathrm{~K}^{4} / \mathrm{W}$ (for the speed of sound of transverse phonons), which is also much less than the measured resistivity at any temperature. The estimated value of $27 \mathrm{~cm}^{2} \mathrm{~K}^{4} / \mathrm{W}$ is even 18 times lower than the measured resistivity at $396 \mathrm{mK}$ stamp temperature. In fig. 6.11 one can see a large influence of the thin layer of $\mathrm{Ti}$ and the neighbouring Au-Ti interface, when titanium becomes normal conducting above $390 \mathrm{mK}$. The decrease of thermal resistivity from about $475 \mathrm{~cm}^{2} \mathrm{~K}^{4} / \mathrm{W}$ to $420 \mathrm{~cm}^{2} \mathrm{~K}^{4} / \mathrm{W}$ demonstrates the high influence of such a thin layer on the thermal resistivity of the whole setup.

The theoretical value of the total interface resistance for the sandwich with titanium and gold is lower than that of the first sandwich, because of a smaller dissimilarity between the neighbouring materials. A plot of the thermal resistivities of both sandwich setups in all measured configurations, without the application of an external magnetic field, is presented in figure 6.12. Contrary to the theoretical prediction of the AMM, the measured thermal resistivity of the sandwich with thin layers of superconducting titanium and indium is much higher than the thermal resistivity of all the measured configurations of the copper - indium - sapphire sandwich. It indicates that there is another source of thermal resistivity in the sandwich with titanium and gold than just a simple sum of the interface resistivities.

The effect of the reduced thermal conductivity of thin layers, which is visible for the normal conducting case as explained in section 6.2.1, seems to be even stronger in the superconducting case. The phonon wavelength in the $45 \mathrm{~nm}$ 


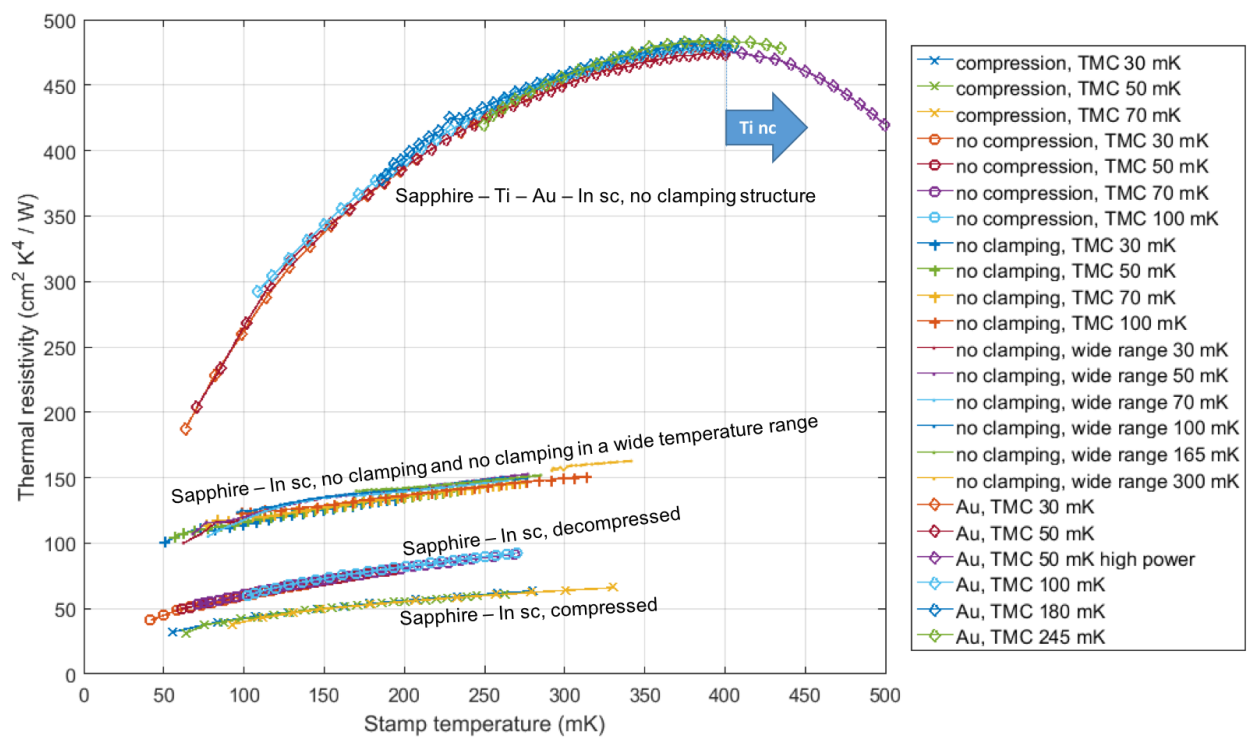

Figure 6.12: Thermal resistivity of both sandwich setups in all measured configurations without the application of an external magnetic field, as a function of the stamp temperature.

layer of titanium is longer than the thickness of this layer already at $5 \mathrm{~K}$. Below $0.39 \mathrm{~K}$ when titanium is superconducting, the wavelength associated with phonons is more than 10 times longer than the thickness of the layer (see figure 5.12). Thus at this temperature a thin layer of titanium, having dielectric-like thermal properties, creates a serious bottle-neck for the heat transfer. Below approx. $0.3 \mathrm{~K}$ the dominant phonon wavelength in gold also exceeds the thickness of this layer, which decreases the effective thermal conductivity even further. The 6 to 18 times higher than expected thermal resistivity could be explained among others by the highly reduced conductivity of the thin layers.

The copper - indium sc interface, which is most probably responsible for the twice higher than expected thermal resistivity of the copper - indium sc sapphire sandwich, as described in section 6.1.3, is also present in this setup. As indicated by the STEM analysis presented in section 3.1.3 [68], the layer of titanium has a sharp interface with sapphire, but there should be at least a few atomic layers of $\mathrm{TiO}$ responsible for the attachment of titanium to sapphire. Moreover, there may be an ultra-thin layer of an intermetallic compound created between titanium and gold. It may be that this intermetallic layer creates an additional thermal resistance. The outer (i.e. further from the sapphire disk) surface of the layer of gold is not perfectly flat, but rather wavy. It is questionable, whether the indium foil managed to fill all the grooves between the waves, or if the contact area in a micro-scale is much smaller than the nominal area. The sandwich was assembled in an air atmosphere, not in vacuum, so there 
may also be tiny amounts of air trapped in the grooves, that could additionally degrade the interface quality by inducing a local oxidation.

\subsubsection{Comparison with the model}

\section{$\mathrm{Cu}-$ In nc - Au - Ti nc - sapphire}

As presented in previous sections of this chapter the theoretical estimations of the Kapitza resistivity of the sapphire - indium and sapphire - titanium interfaces in the normal and the superconducting states do not differ significantly, in contrast to the obtained measurement results. Therefore, the titanium nc - sapphire - titanium nc connection will be treated as the indium nc - sapphire - indium nc connection including the parallel plates assumption, which results from section 6.1.4. The electrical analogon of that configuration is presented in figure 5.10. The characteristic conductivity $\alpha_{\mathrm{Ti}}$ nc and the exponent $n_{\mathrm{Ti} \text { nc }}$ of the temperature dependence of the thin layer of titanium satisfying equation (5.4) is found from the measurement data. A set of equations as explained in section 6.1.4 has been solved and the obtained values were averaged (see $\alpha_{\mathrm{Tinc}}^{\prime}=\alpha_{\mathrm{Ti} \mathrm{nc}} / l_{\mathrm{Ti}}=1 / \kappa_{\mathrm{Ti} \mathrm{nc}}$ figure 6.13), giving as a result the conductivity of the $45 \mathrm{~nm}$ layer of titanium: $\alpha_{\mathrm{Ti} \mathrm{nc}}^{\prime}=\alpha_{\mathrm{Ti} \mathrm{nc}} / l_{\mathrm{Ti}}=1 / \kappa_{\mathrm{Ti} \mathrm{nc}}=$ $1 / 33.78=0.0296 \mathrm{~W} /\left(\mathrm{cm}^{2} \mathrm{~K}^{\mathrm{n}}\right)$ and $n_{\mathrm{Ti} \mathrm{nc}}=2.453$. The corresponding conductivity of "bulk" titanium would be $\alpha_{\mathrm{Ti} \mathrm{nc}}=0.0296 \mathrm{~W} /\left(\mathrm{cm}^{2} \mathrm{~K}^{\mathrm{n}}\right) \cdot 45 \mathrm{~nm}=$ $1.332 \cdot 10^{-7} \mathrm{~W} /\left(\mathrm{cm} \mathrm{K}^{\mathrm{n}}\right)$, which is of course not physical, as the strong reduction of conductivity is only an effect visible in thin layers and it cannot be treated as a property of a bulk material. Therefore $\alpha_{\mathrm{Ti} \text { nc }}^{\prime}$, i.e. the value characteristic for a thin layer of $45 \mathrm{~nm}$, was summarised in table 6.4 as a result obtained from the model. The exponent of the titanium conductivity equal to $n_{\mathrm{Ti} \mathrm{nc}}=2.453$ indicates that there is a significant contribution from the lattice side to the conductivity of the thin layer of titanium, even in the normal conducting state.

Table 6.4: The theoretical and calculated values of the characteristic resistivity and thin layer conductivity present in the $\mathrm{Cu}-\mathrm{In} \mathrm{nc}-\mathrm{Au}-\mathrm{Ti} \mathrm{nc}$ - sapphire setup.

\begin{tabular}{|c|c|c|}
\hline Value & Theoretical & Calculated from the model \\
\hline$\kappa_{\text {sapphire disk }}^{\text {parallel plates }}$ & $18.27 \mathrm{~cm}^{2} \mathrm{~K}^{4} / \mathrm{W}$ & $70.92 T+20.34 \mathrm{~cm}^{2} \mathrm{~K}^{4} / \mathrm{W}$ \\
$\kappa_{\mathrm{Ti} \mathrm{nc}}=l_{\mathrm{Ti}} / \alpha_{\mathrm{Ti} \mathrm{nc}}$ & $1.3 \cdot 10^{-3} \mathrm{~cm}^{2} \mathrm{~K}^{2} / \mathrm{W}$ & $33.78 \mathrm{~cm}^{2} \mathrm{~K}^{\mathrm{n}} / \mathrm{W}$ \\
$\alpha_{\mathrm{Ti} \mathrm{nc}}^{\text {bulk }}$ & $3.45 \cdot 10^{-3} \mathrm{~W} /\left(\mathrm{cm} \mathrm{K}^{2}\right)$ & $1.332 \cdot 10^{-7} \mathrm{~W} /\left(\mathrm{cm} \mathrm{K}^{\mathrm{n}}\right)$ \\
$n_{\mathrm{Ti} \mathrm{nc}}$ in $\lambda=\alpha T^{n}$ & 1 & 2.453 \\
\hline
\end{tabular}

The high resistance value of the thin layer of $\mathrm{Ti}$ (both $\kappa$ and $n$ ) may be also an effect of the uncertain nanometer layer of an oxide responsible for the adhesion of titanium to sapphire 97,105. Perhaps better distinguishing between the effects of the interface and the thin layer properties (unfortunately rather 
difficult to realize) would result in an increase of the values of $\kappa$ corresponding to the interface effects and a decrease of $\kappa$ and $n$ of the thin layer.

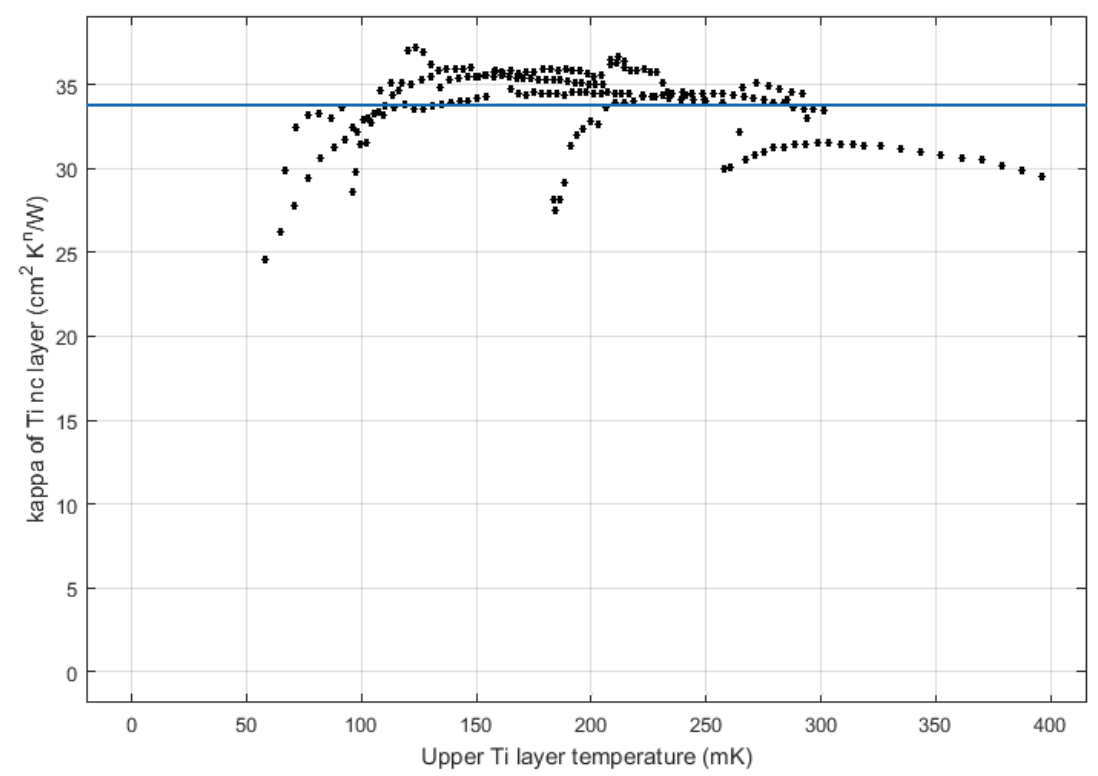

Figure 6.13: The calculated value of $\kappa_{\mathrm{Ti} \mathrm{nc}}=l_{\mathrm{Ti}} / \alpha_{\mathrm{Ti}}$ nc as a function of the temperature of its thin layer for exponent $n_{\mathrm{Ti} \mathrm{nc}}=2.453$. The averaged value equals $33.78 \mathrm{~cm}^{2} \mathrm{~K}^{\mathrm{n}} / \mathrm{W}$.

\section{$\mathrm{Cu}$ - In sc - Au - Ti sc - sapphire}

The electric analogon of the last analysed configuration is presented in figure 5.14. The Ti sc - sapphire - Ti sc connection is modelled as In nc - sapphire - In nc including the parallel plates assumption, because the theoretical estimations of the sapphire - Ti and sapphire - In resistivity do not differ a lot. Interfaces between metals and superconductors, i.e. $\mathrm{Cu}-\mathrm{In} \mathrm{sc}, \mathrm{In} \mathrm{sc}-\mathrm{Au}$ and $\mathrm{Au}$ - Ti sc, are modelled as the $\mathrm{Cu}$ - In sc interface described in section 6.1.4. The layers of indium foil are also modelled as in section 6.1.4. Since gold is known to be a good thermal conductor, and the resistance corresponding to its thin layer calculated in a classical way is negligible, it will be skipped in the model. Instead, the thin layer of superconducting titanium will have a new value of $\kappa$ and $n$ assigned, corresponding to its superconducting state. In the equations the properties of titanium were modelled as resistivity, not conductivity, what makes the contribution of titanium to the total sandwich resistivity more visible.

The calculated, averaged value of the exponent equals to $n_{\mathrm{Ti} \mathrm{sc}}+1=3.855$ and it decreases slightly above $350 \mathrm{mK}$, see figure 6.14, close to the point 
where titanium becomes normal conducting and electrons start playing a role in the heat transfer. The resulting resistivity for $n_{\mathrm{Ti} \mathrm{sc}}=2.855$ is presented in figure 6.15 and follows the shape of the plot in figure 6.11. The value of resistivity rises to the maximum of $170 \mathrm{~cm}^{2} \mathrm{~K}^{\mathrm{n}} / \mathrm{W}$ at approx. $330 \mathrm{mK}$. At that temperature the resistivity starts decreasing in accordance with the decrease of the exponent. The temperature is a bit lower than the nominal temperature of the superconducting transition of titanium. It may be that the internal stresses in the thin layer of sputtered titanium, resulting from the differences in sapphire and titanium lattice spacing, the presence of the Earth's magnetic field or a certain amount of impurities (mainly oxides) cause a decrease in the temperature of the superconducting transition of titanium from $0.39 \mathrm{~K}$ to approximately $0.33 \mathrm{~K}-0.35 \mathrm{~K} 106$.

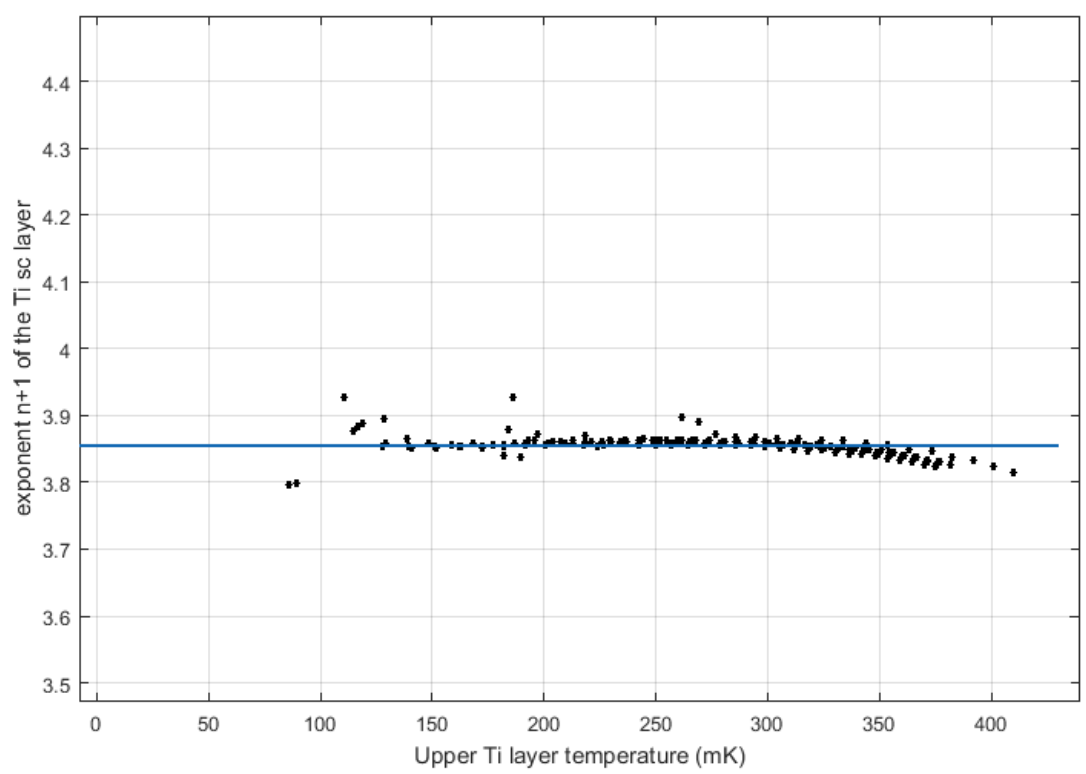

Figure 6.14: The calculated values of the exponent describing the temperature dependence of the resistance of the thin layer of $\mathrm{Ti} s \mathrm{sc}$ in a form $R_{\mathrm{Ti} \mathrm{sc}}=\kappa_{\mathrm{Ti} \mathrm{sc}} T^{-n}$. Averaging gives a value of $n_{\mathrm{Ti}} \mathrm{sc}+1=3.855$, i.e. $n_{\mathrm{Ti}} \mathrm{sc}=2.855$.

The high value of the calculated resistivity reflects the diminished heat transfer across the thin layer of titanium, and there are several reasons contributing to these limitations. The first is the fact that the dominant phonon wavelength exceeds the thickness of the layer, what can decrease the thermal conductivity of the thin layer several times, as explained in section 5.4. It is very probable that the ultra-thin interlayers of compounds responsible for the adhesion of gold to titanium and titanium to sapphire [97, 105, 107] also limit the heat transfer in the sandwich, by creating other "interfaces" and disturbing 
the continuity of the lattice structure.

It is clear that the presence of the thin layers of titanium and gold creates a serious bottleneck for heat transfer and therefore the deposition of these layers should be avoided on the bottom surface of the electrodes, anchoring them to the cold source in the AEgIS experiment. Indium shows much lower thermal resistivities, especially in the normal conducting state, and it enables the removal of the clamping structure.

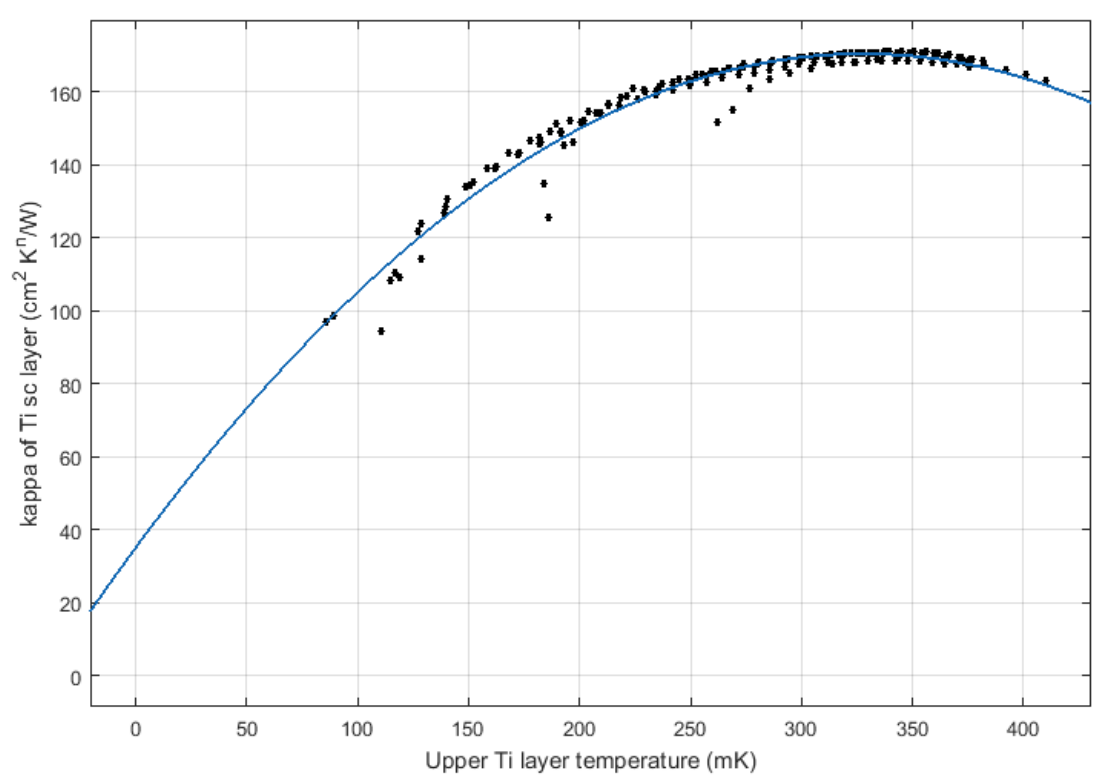

Figure 6.15: The calculated values of the $\kappa_{\mathrm{Ti}}$ sc describing the temperature dependence of the resistance of the thin layer of Ti sc in a form $R_{\mathrm{Ti} \mathrm{sc}}=\kappa_{\mathrm{Ti} \mathrm{sc}} T^{-n}$ for $n=2.855$.

\subsection{Transient measurements in the low temperature range from $3 \mathrm{~K}$ to $30 \mathrm{~K}$}

The thermal conductivity and diffusivity of both sandwich setups was measured in the temperature range from $3 \mathrm{~K}$ to $30 \mathrm{~K}$ in the CERN Cryolab pulse tube refrigerator. The setup was mounted on the cold head of the cryocooler with two Cernox sensors: on the stamp and on the platform. A layer of indium below the sensors was used to improve their thermal contacts. A sample heater (SHT) was glued to the stamp and the power was supplied with a function generator. The setup hanging on the cryocooler is presented in figure 3.10 . Both setups were measured without the clamping structure present. At each base temperature the thermal diffusivity was determined with the amplitude method described 
in section 4.4 .1 for thermal excitation frequencies from $0.02 \mathrm{~Hz}$ to $12 \mathrm{~Hz}$.

\subsubsection{Thermal diffusivity of the copper - indium - sapphire sandwich setup}

The thermal diffusivity of the copper - indium - sapphire sandwich sample as a function of frequency for temperatures from $2.8 \mathrm{~K}$ to $8.4 \mathrm{~K}$ is presented in figure 6.16 [2] The size of the error bars at the level of the pleateau (around $1 \mathrm{~Hz}$ ), not presented here for clarity, is comparable with error bars in figure 6.20. As in the steady state measurements, the accuracy of the measurement decreases along with a decrease of the measured temperature differences, i.e. towards the high temperature end for measurements performed with the same power at each base temperature (as in figure 6.20), and towards high frequencies. Figure 6.17 shows how $(\Delta T)^{-1}$ increases (i.e. the measured temperature difference decreases) with an increase of frequency and with an increase of the platform base temperature. The smaller and smaller temperature gradients cause an increase of the measurement error.

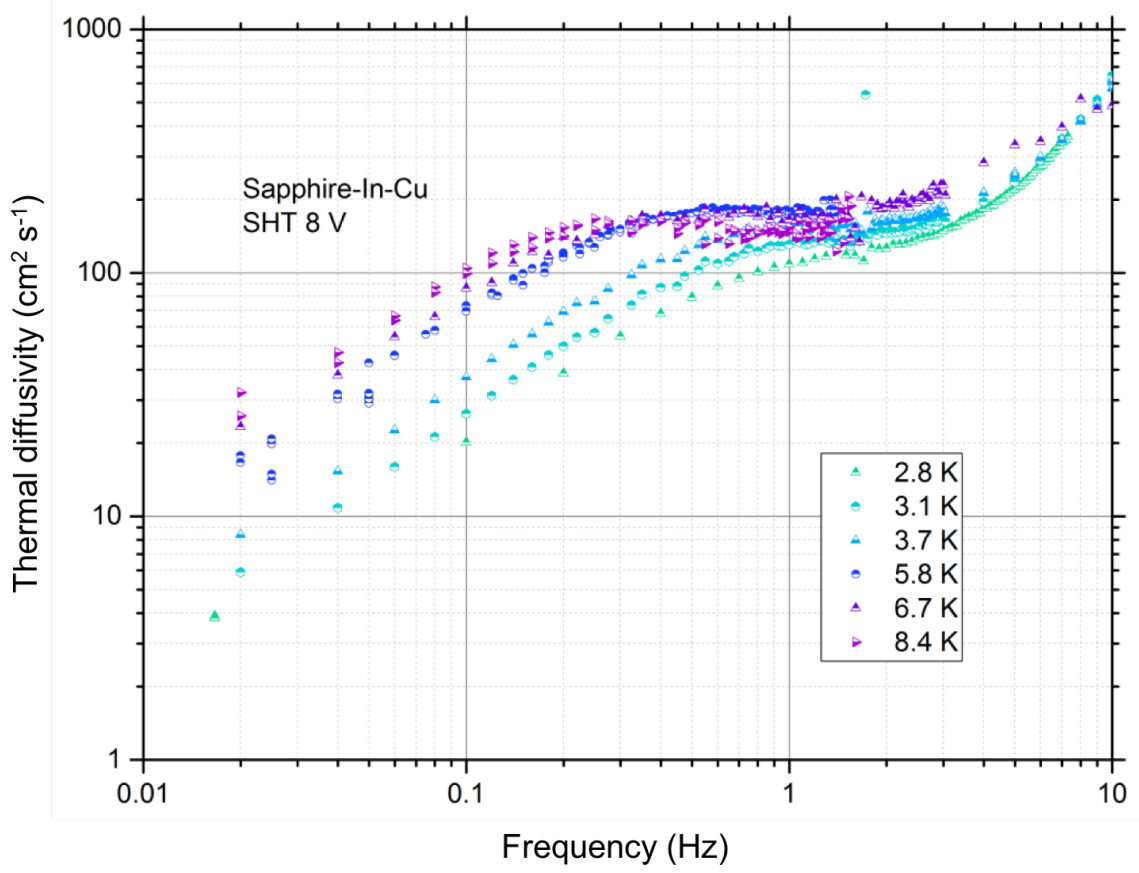

Figure 6.16: Thermal diffusivity of the copper - indium - sapphire sandwich setup as a function of frequency for platform temperatures from $2.8 \mathrm{~K}$ to $8.4 \mathrm{~K}[2]$. The peak-to-peak voltage of a sine wave supplied by the function generator to the sample heater (SHT) of $100 \Omega$ resistance was $8 \mathrm{~V}$.

The distance between the temperature sensors on the stamp and the platform necessary for the diffusivity measurements equals $19.75 \mathrm{~mm}$. The singular point at $1.7 \mathrm{~Hz}$ having a much higher diffusivity than the rest of the $3.1 \mathrm{~K}$ series, 


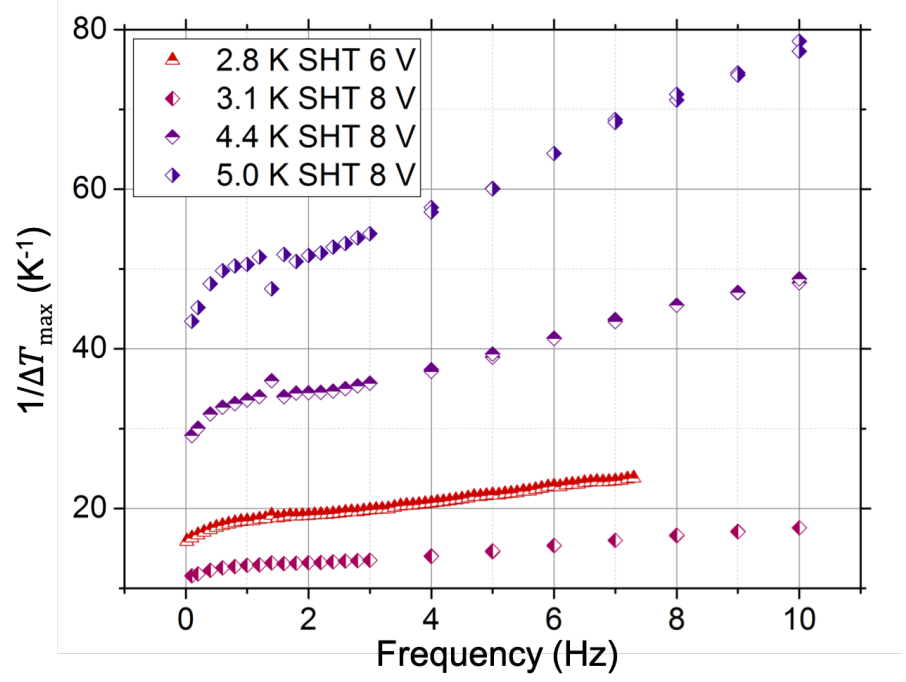

Figure 6.17: An increase of $1 / \Delta T$ (i.e. decrease of the measured temperature difference) as a function of frequency for several platform temperatures. The peak-to-peak voltage of a sine wave supplied by the function generator to the sample heater (SHT) of $100 \Omega$ resistance was $8 \mathrm{~V} 72$.

and the disturbance around $1.75 \mathrm{~Hz}$ visible for all base temperatures, are the result of a resonance with the operating frequency of the pulse tube cryocooler. As already known from the validation of the measurement method described in chapter 4 , there is a temperature dependent threshold frequency $f_{c_{1}}$, below which a proper diffusivity measurement is not possible. For the copper - indium - sapphire sandwich the first threshold frequency equals to around $1 \mathrm{~Hz}$ at $2.8 \mathrm{~K}$, and $0.2 \mathrm{~Hz}$ for $8.4 \mathrm{~K}$. Unexpectedly, a second threshold frequency $f_{c_{2}}$ appears around $2 \mathrm{~Hz}$, above which the diffusivity starts rising again. The influence of the increased measurement error for small $\Delta T$ is causing such a behaviour in the extracted thermal diffusivity data 72 .

Measurements at frequencies higher than $10 \mathrm{~Hz}$ are impossible because of a high noise in the temperature signal. Therefore, the thermal diffusivity from the plateau between $0.3 \mathrm{~Hz}$ and $2 \mathrm{~Hz}$, where the diffusivity takes a value of approx. $100-200 \mathrm{~cm}^{2} / \mathrm{s}$, was taken as a characteristic value at a given temperature. Higher base temperatures have been measured as well, and all of them show an increase of the thermal diffusivity with an increase of frequency above the pleateau 72 .

The thermal diffusivity of the sandwich as a function of temperature for various peak-to-peak voltages supplied to the heater is presented in figure 6.20. It allows a direct comparison with the $\mathrm{Ti}-\mathrm{Au}$ sandwich results, discussed in the next section. 


\subsubsection{Thermal diffusivity of the copper - indium - gold - titanium - sapphire sandwich setup}

The copper - indium - gold - titanium - sapphire sandwich setup was measured on the cryocooler in two configurations: with and without the cryocooler cold tip attenuator, described in section 3 , installed between the cold head and the sandwich. The principal purpose of the attenuator is the elimination of the resonance peak around $1.7 \mathrm{~Hz}$. The thermal diffusivity of the second sandwich setup measured without the attenuator is presented in figure 6.18.

The distance between the stamp and platform sensors equals to $27.75 \mathrm{~mm}$. The resonance peak around $1.7 \mathrm{~Hz}$ is not shown. The plateau of thermal diffusivity between the two threshold frequencies is visible at the lowest temperatures as in figure 6.16. When the temperature increases, the plateau gradually disappears and the value of diffusivity is monotonically increasing with frequency, creating a limit for the experimental determination of the diffusivity towards higher temperatures.

The installation of the attenuator described in section 3.3 .3 significantly changed the obtained values of the thermal diffusivity, especially in the low frequency range, as shown in figure 6.19. For the lowest measured temperatures, the first threshold frequency shifts to much lower values and the thermal diffusivity has a constant or even slightly increasing value towards low frequencies. At higher temperatures the diffusivity value decreases with a decrease of frequency. A modification of the measurement stand - the installation of the attenuator - influenced the results. It is difficult to decide which value should be taken as representative for the diffusivity of the sandwich setup. The value from the plateau was taken, but since the plateau disappears at temperatures above $10 \mathrm{~K}$, the precision of the diffusivity readout is low.

The thermal diffusivity of both sandwich setups as a function of temperature, with and without the attenuator, for various heating powers are compared in figure 6.20 [72]. The results show a good agreement for all peak-to-peak voltages $(4 \mathrm{~V}, 8 \mathrm{~V}, 10 \mathrm{~V}, 16 \mathrm{~V}, 20 \mathrm{~V})$ of the sine waves supplied to the $100 \Omega$ heater. Only the low temperature part of the plot below $5 \mathrm{~K}$, for the highest heating power of $16 \mathrm{~V}$ and $20 \mathrm{~V}$, without the attenuator, deviates from the rest. The diffusivity in figure 6.20 is plotted as a function of temperature, which is the mean temperature between the stamp and the platform. It may happen that for high heating powers, the temperature gradient across the sample is slightly different, despite the same mean temperature, what could explain the deviation of results. The peak of the thermal diffusivity of the copper - indium sapphire sandwich is at $6 \mathrm{~K}$, and of the copper - indium - gold - titanium sapphire sandwich at $4 \mathrm{~K}$. Both curves merge at approximately $3 \mathrm{~K}$, despite the expectation that the thermal diffusivity of the setup which has a higher thermal resistivity - the sandwich with titanium and gold - would be lower. In the next section, the results are related to the theory and the parameters 


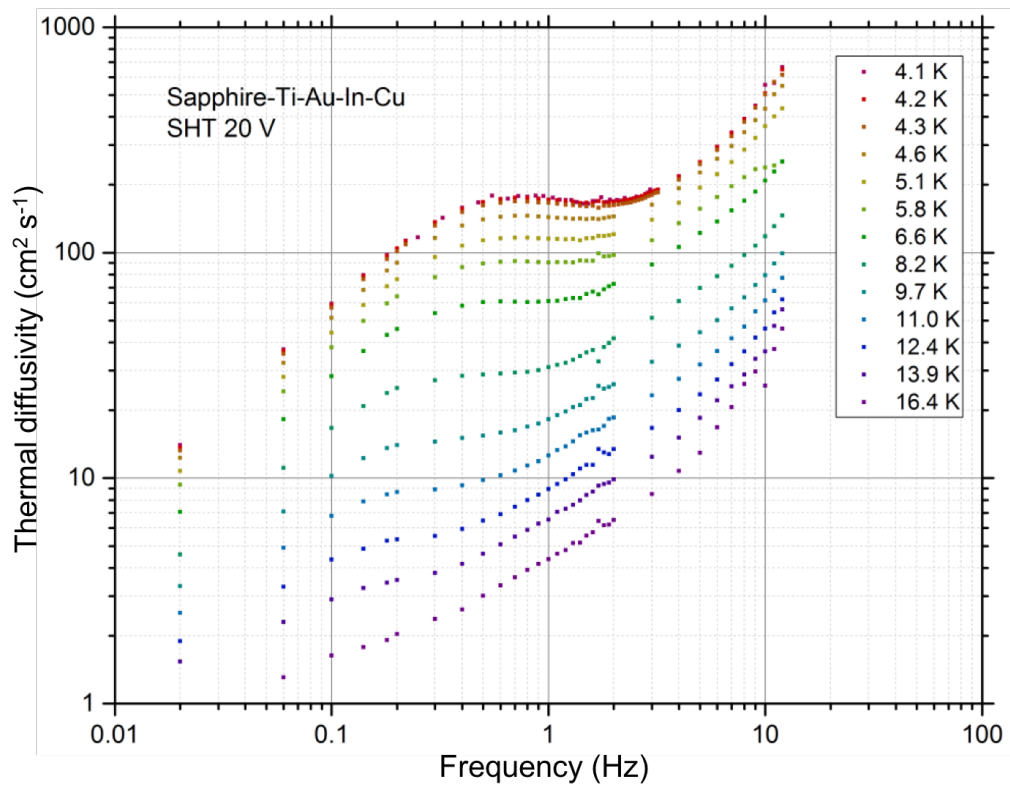

Figure 6.18: Thermal diffusivity of the $\mathrm{Cu}$ - In - $\mathrm{Au}$ - Ti - sapphire sandwich setup as a function of frequency at different platfrom temperatures without the attenuator installed 72. The peak-to-peak voltage of the sine wave supplied by the function generator to the $100 \Omega$ heater was $20 \mathrm{~V}$.

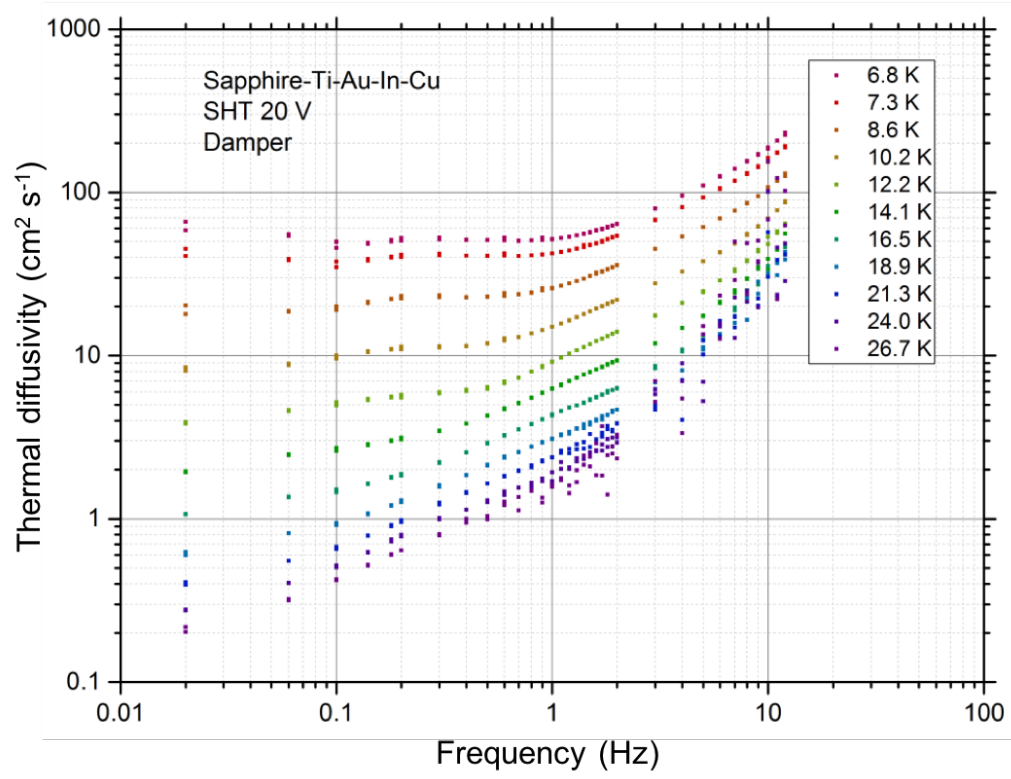

Figure 6.19: Thermal diffusivity of the $\mathrm{Cu}$ - In - Au - Ti - sapphire sandwich setup as a function of frequency at different platform temperatures with the attenuator installed 72 . 


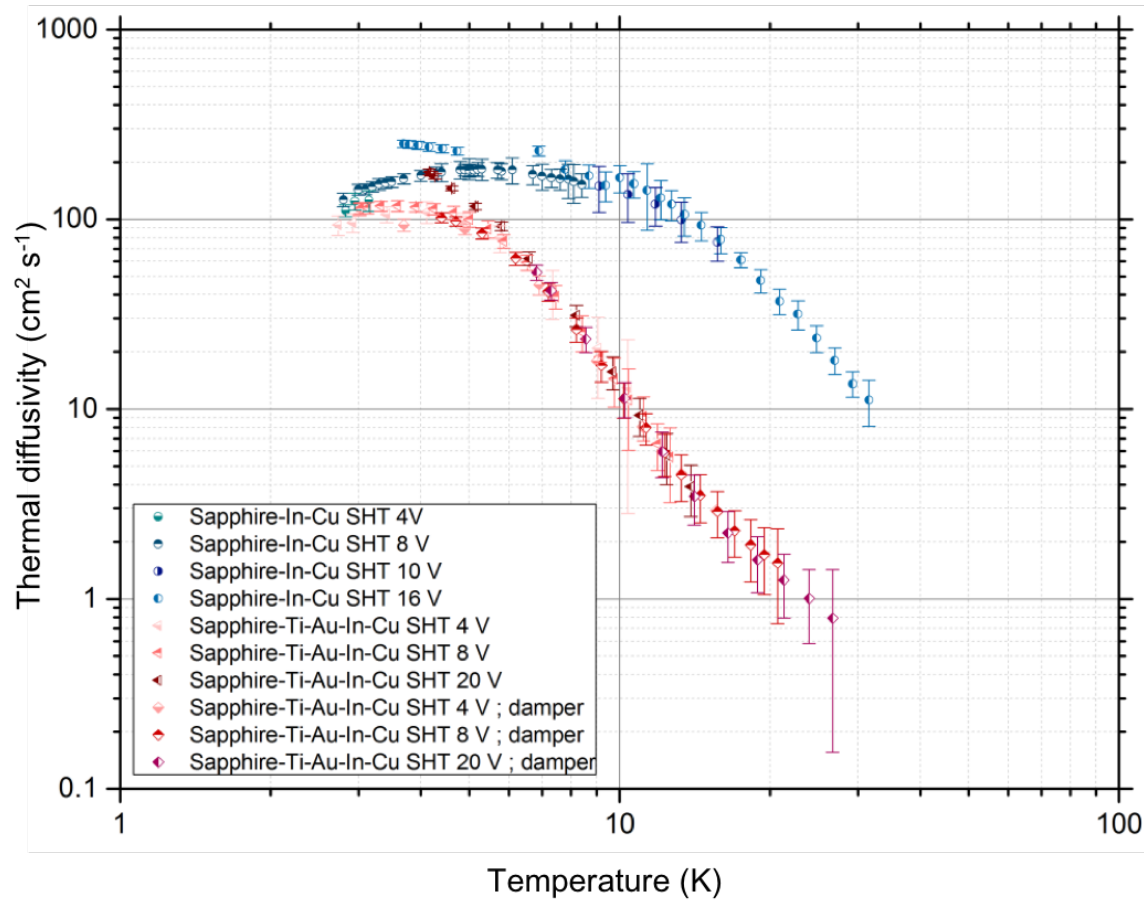

Figure 6.20: Thermal diffusivity of both sandwich setups as a function of temperature, for various peak-to-peak voltages supplied to the $100 \Omega$ heater $(4 \mathrm{~V}, 8 \mathrm{~V}, 10 \mathrm{~V}, 16 \mathrm{~V}, 20 \mathrm{~V}) 72$. The temperature on the horizontal axis is the average temperature between the platform and the stamp.

influencing the thermal diffusivity are analysed.

\subsubsection{Theoretical estimation of the low temperature thermal conductivity and thermal diffusivity}

To check whether the value of the diffusivity taken from the plateau is realistic, a theoretical estimation is made. The thermal diffusivity can be calculated as in eq. (2.29). The densities and low temperature heat capacities of all the materials can be found in literature $8,9,12,13,93,108,111$. To make the estimation as accurate as possible, an effective measured thermal conductivity of the whole sandwich in steady state conditions is used instead of taking literature values of separate materials. The low temperature thermal conductivity of both sandwich setups containing all the thin layers and interfaces was measured in-situ on the cryocooler. That measurement was performed by applying a constant heating on the stamp, and measuring the temperatures on the stamp and the platform. The effective conductivity of the sandwich was then calculated as:

$$
\lambda_{\text {sandwich }}^{\mathrm{eff}}=\frac{\dot{Q} \cdot l}{A_{\min } \cdot \Delta T},
$$


where $\dot{Q}$ is the applied heat load, $l$ is the distance between the sensors, $A_{\min }$ is the minimum cross-section of the sandwich, and $\Delta T$ is the measured temperature difference. The minimum cross-section of both sandwiches equals to $3.14 \mathrm{~cm}^{2}$. The measured thermal conductivity of both sandwich setups in the temperature range from $3 \mathrm{~K}$ to $60 \mathrm{~K}$ is presented in figure 6.21 [72]. As in the ultra-low temperature steady state measurements, see figure 6.10, the thermal conductivity of the sandwich with titanium and gold is lower than the conductivity of the sandwich with only indium. The peak of the copper - indium sapphire sandwich conductivity is at around $30 \mathrm{~K}$, and the copper - indium gold - titanium - sapphire sandwich at around $7 \mathrm{~K}$. Both curves merge towards the low temperature end of the plot, as in the thermal diffusivity measurements. The error bars are within the size of the plotted points.

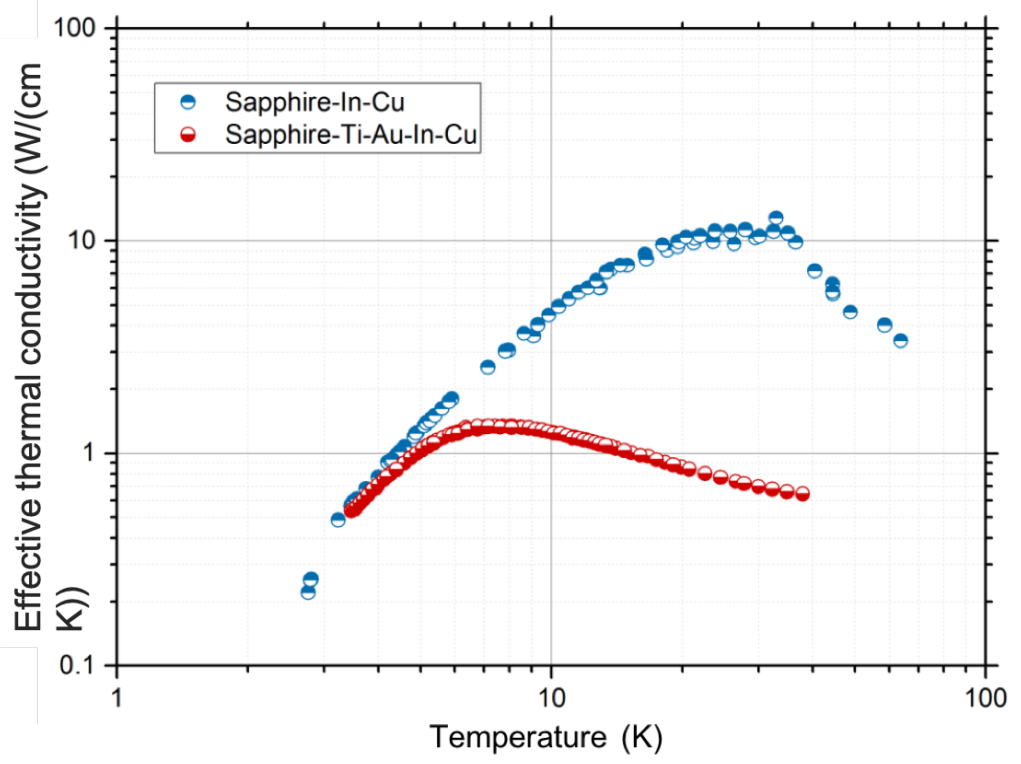

Figure 6.21: Effective low temperature thermal conductivity of both sandwich setups measured on the cryocooler 72 . The error bars are within the size of the plotted points.

The thermal resistance of the setup can be also calculated from the literature values as a linear combination of resistances of individual elements and interfaces:

$$
R_{t o t}=\Sigma_{i} R_{i}+\Sigma_{j} R_{\text {interface }_{j}}
$$

where $\Sigma_{i} R_{i}$ is the sum of resistances of each layer present in the sandwich, and $\Sigma_{j} R_{\text {interface }_{j}}$ is the sum of interface resistances calculated according to equation 2.16). The resistance of each layer can be calculated as:

$$
R_{i}=\frac{x_{i}}{\lambda_{i} \cdot A_{i}}
$$


where $x_{i}$ is the thickness of each layer, $\lambda_{i}$ the conductivity of each layer, and $A_{i}$ is the cross-section. The cross-section of the setup changes along the sandwich, see e.g. figure 3.1. The diameter of the sapphire is $20 \mathrm{~mm}$, whereas the width of the copper platform equals $50 \mathrm{~mm}$. To simplify the problem the minimum cross-section of $3.14 \mathrm{~cm}^{2}$ is taken.

The significance of the thermal interface resistance in the low temperature range from $3 \mathrm{~K}$ to $60 \mathrm{~K}$ can be verified by comparing the theoretically estimated $1 / R_{\text {tot }}$ with the measured value of conductivity. The low temperature thermal conductivity of all the materials according to literature data, and the estimated thermal conductivity of the copper - indium - sapphire sandwich is presented in figure 6.22. Since the interface resistance even in the ultra-low temperature range does not follow exactly the temperature dependence described by eq. (2.21), i.e. $R_{\text {interface }} \propto T^{-3}$, but rather $R_{\text {interface }} \propto T^{n}$, where $n \in(-3 ;-2)$, the theoretical estimation of the low temperature thermal conductivity, plotted in figure 6.22, was done for three cases 72 :

(a) the estimated total resistivity is a sum of resistivity values of all the materials;

(b) the estimated total resistivity is a sum of resistivity values representing materials and interfaces in between, with an interface resistance $R_{\text {interface }} \propto T^{-3}$

(c) the estimated total resistivity is a sum of resistivity values representing materials and interfaces in between, with an interface resistance $R_{\text {interface }} \propto T^{-2.5}$.

The dashed and dotted lines in fig. 6.22 represent the literature values of bulk thermal conductivities of all the constituents of the first sandwich setup. These are for reference only concerning the temperature dependence. Absolute levels cannot be compared to measurement data because of considering all cross-sectional areas to be equal to the minimum area in the sandwich. One can see that the green line, corresponding to case (a), follows quite closely the thermal conductivtity of copper, except for the low temperature end. Case (b), represented by the blue line, has a slightly lower thermal conductivity, and as expected the influence of the interface resistivity becomes more significant when the temperature decreases. Changing the interface resistance temperature dependence from $T^{-3}$ to $T^{-2.5}$ (case (c), represented by the violet line), gives the lowest estimation of the thermal conductivity, and follows the measurement results very closely, except for a certain deviation at the high temperature end above $35 \mathrm{~K}$, which can be explained by lower measurement precision at higher temperatures 72 . The temperature dependence of the thermal boundary resistance with an exponent higher than -3 was also observed and discussed by other authors [112. The maximum thermal conductivity for all the cases equals 


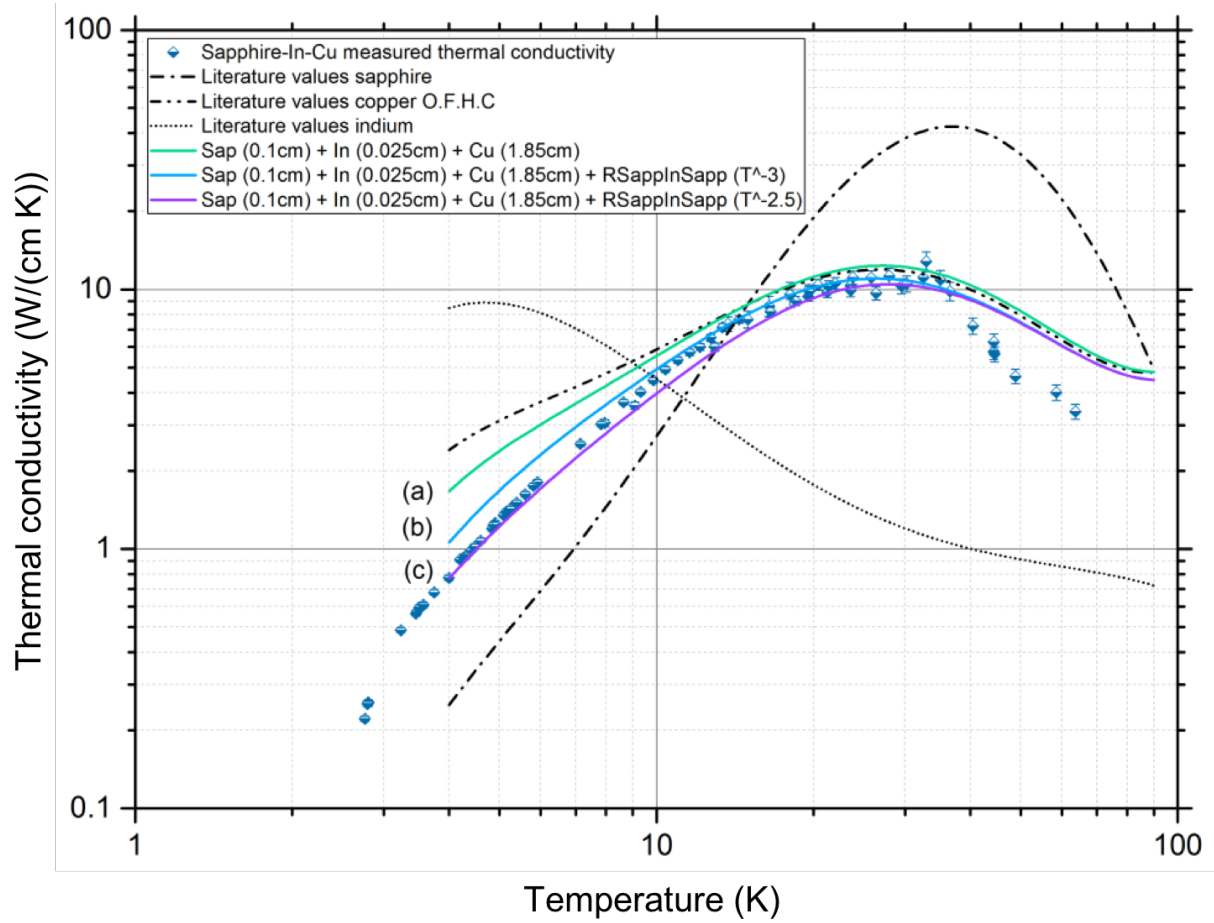

Figure 6.22: Thermal conductivity of the individual materials the sandwich consists of, and the resistivity of the whole sandwich estimated with and without taking into account interface resistances 72 .

around $10 \mathrm{~W} /(\mathrm{cmK})$ at around $28 \mathrm{~K}$, and agrees with the measured value. The more important - low temperature - behaviour of the setup is predicted very well by case (c), what also justifies the use of $A_{\min }=3.14 \mathrm{~cm}^{2}$ as the critical cross-section of the whole setup.

With the measured and modelled thermal conductivity one can now calculate the total thermal diffusivity of the sandwich as 72 :

$$
a_{\text {sandwich }}=\frac{l}{A \cdot R_{t o t} \cdot c_{t o t} \cdot \rho_{\text {tot }}},
$$

where the total resistance is calculated according to equation $(6.3)$, the crosssection for the heat transport is assumed to be constant and equal to $A=$ $A_{\min }=3.14 \mathrm{~cm}^{2}, l$ is the total thickness of the setup, equal to the distance between the temperature sensor on the stamp and that on the platform. The multiplication of the specific heat and density $c_{t o t} \cdot \rho_{t o t}$ of the whole sandwich depending on the thickness of each layer is calculated in the following way:

$$
c_{t o t} \cdot \rho_{t o t}=\Sigma_{i} \frac{x_{i}}{l} \cdot c_{i} \cdot \rho_{i} .
$$

The specific heat of sapphire, gold, indium, and copper was taken from literature data 8, 9, 93, 108]. The $45 \mathrm{~nm}$ layer of titanium was neglected in eq. (6.6) 
as its contribution to the total heat capacity is vanishing. The comparison of the estimated and measured thermal diffusivity for both sandwich setups is presented in figure 6.23 .

For both setups the curves of measured and estimated thermal diffusivity have a very similar shape, and the peak of diffusivity occurs at the same temperature of approx. $5 \mathrm{~K}$ for the copper - indium - sapphire sandwich and approx. $4 \mathrm{~K}$ for the copper - indium - gold - titanium - sapphire sandwich. However, the measured value of diffusivity is for both setups several times lower than predicted.
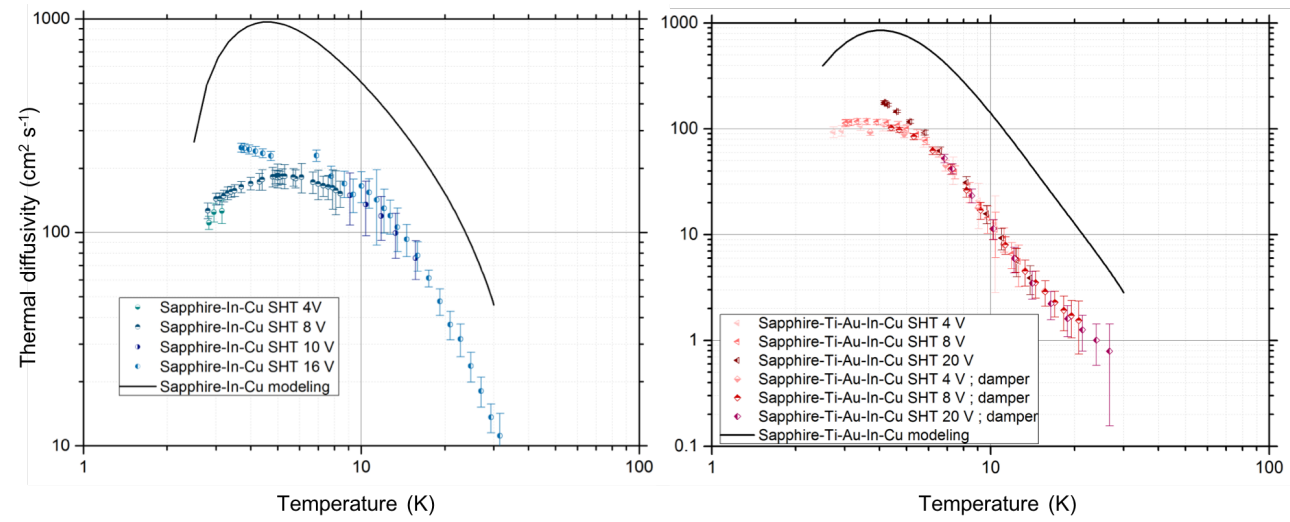

Figure 6.23: A comparison of the thermal diffusivity values as a function of temperature of the $\mathrm{Cu}$ - In - sapphire setup (left) and $\mathrm{Cu}$ - In - Au - Ti - sapphire setup (right), both estimated according to eq. 6.5 and measured. The measurement was done for various peak-to-peak voltages applied to the heater 72 .

Since the thermal conductivity taken for the diffusivity estimation was measured, the only error can be coming from incorrect estimation of the total heat capacity, especially the volume of the material actually taking part in heat transfer and storage. It is discussible whether the total volume of copper should be taken in to account or only the part of the setup in a direct line of the heat flow, corresponding to the smallest cross-section. The total volume of copper in the stamp and the platform is 3.3 times bigger than the volume in the direct line of the heat flow for the copper - indium - sapphire sandwich, and 2.7 times bigger for the sandwich with titanium and gold, see figure 6.24 [72]. The shape of all the elements was dictated by the necessity of providing a compression force for measurements in the DR. Including in the calculations the geometry factor reflecting the amount of material really participating in the transient heat transfer gives a good agreement between the measured and modelled values for the $\mathrm{Cu}$ - In - sapphire setup as presented in figure 6.25. For the second sandwich setup the modelled value is still approx. 3 times higher than the measured one, but both curves seem to merge towards the low temperature end. 


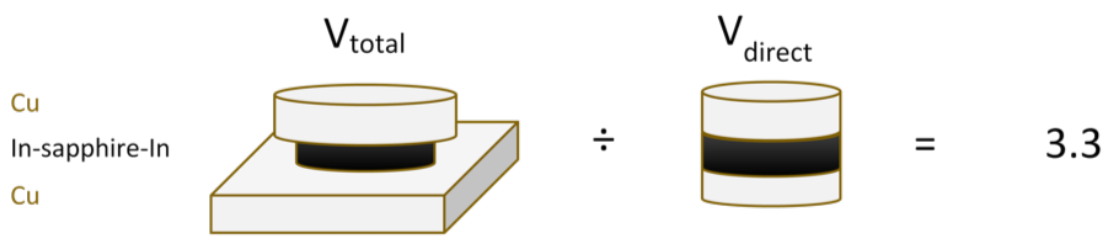

Figure 6.24: Schematics presenting the total amount of material and the amount taking a part in the heat transfer for the $\mathrm{Cu}$ - In - sapphire setup 72 . The geometry factor for this setup equals to 3.3 .
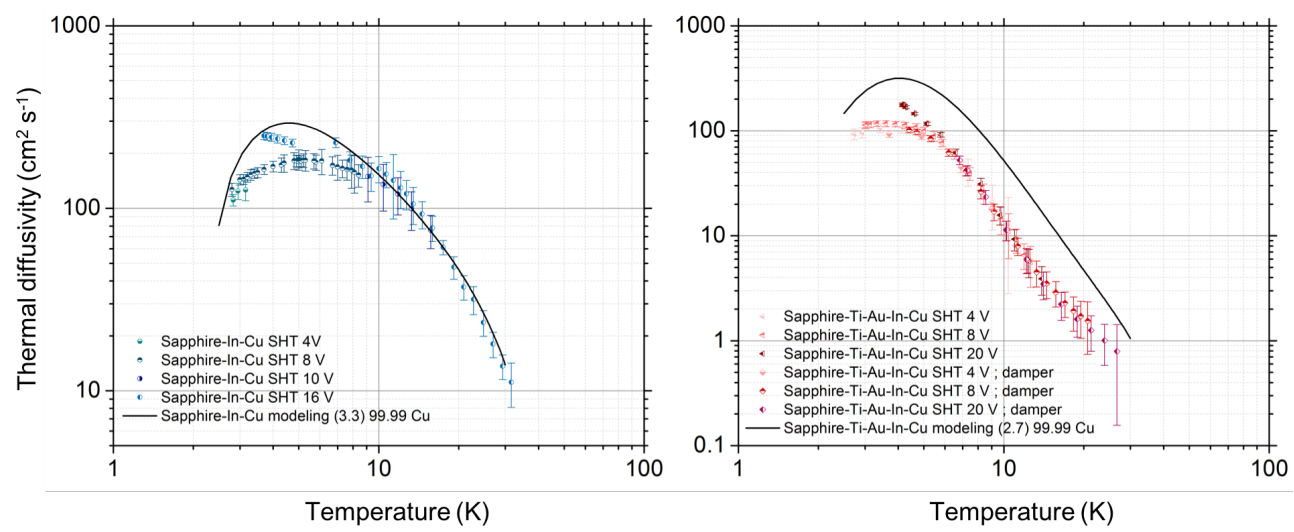

Figure 6.25: A comparison of the thermal diffusivity value as a function of temperature of the $\mathrm{Cu}$ - In - sapphire setup (left) and $\mathrm{Cu}$ - In - Au - Ti - sapphire setup (right), both calculated acc. to eq. 6.5 including the geometry factor and measured. The measurement was done for various peak-to-peak voltages applied to the heater 72 .

\subsection{Transient measurements at ultra-low temperatures}

Diffusivity measurements were performed with the amplitude method also in the ultra-low temperature range. Only the $\mathrm{Au}$ - Ti sandwich was studied with the right conditions of applied heating frequency $f>f_{c 1}$. The focus is on that sandwich due to the fact that there are more thin layers and interfaces present, and therefore it is more likely to deviate from the expected behaviour, based on direct material contributions.

\subsubsection{Thermal diffusivity of the copper - indium - gold - titanium - sapphire sandwich setup}

The copper - indium - gold - titanium - sapphire sandwich was measured at base MC temperatures of $30 \mathrm{mK}, 50 \mathrm{mK}, 100 \mathrm{mK}, 180 \mathrm{mK}$, and $250 \mathrm{mK}$. The maximum frequency of sinusoidal voltage supplied by the function generator was $3 \mathrm{~Hz}$. Above this frequency the measurement became too noisy. The setup was measured with and without magnetic field, switching indium and 
titanium from normal to the superconducting state. In most of the measurements at ultra-low temperatures the plateau was impossible to observe. When the threshold frequencies $f_{c_{1}}$ and $f_{c_{2}}$ become very close to each other, one can observe only an inflection point in the plot of diffusivity as a function of frequency. For some measurements even the inflection point is not observable and the values keep rising with frequency. During transient measurements in the cryocooler, the installation of the attenuator significantly influenced the results at low frequencies. It is possible that a modification of the way the setup is linked to the heat sink - MC would change the results, but it is not applicable in the DR.

With titanium and indium in the normal conducting state, inflection points are observed in the measurements only at base temperatures of $30 \mathrm{mK}$ and $50 \mathrm{mK}$ (figure 6.26). The diffusivity value calculated at these points equals $a_{\text {sandwich }}^{\text {nc }}=0.5 \pm 0.1 \mathrm{~cm}^{2} / \mathrm{s}$ at an average temperature between the stamp and platform of $45 \mathrm{mK}$, and $a_{\text {sandwich }}^{\mathrm{nc}}=1.5 \pm 0.2 \mathrm{~cm}^{2} / \mathrm{s}$ at an average temperature of $93 \mathrm{mK} 72$. The uncertainty has been calculated from the standard deviation around the inflection points.
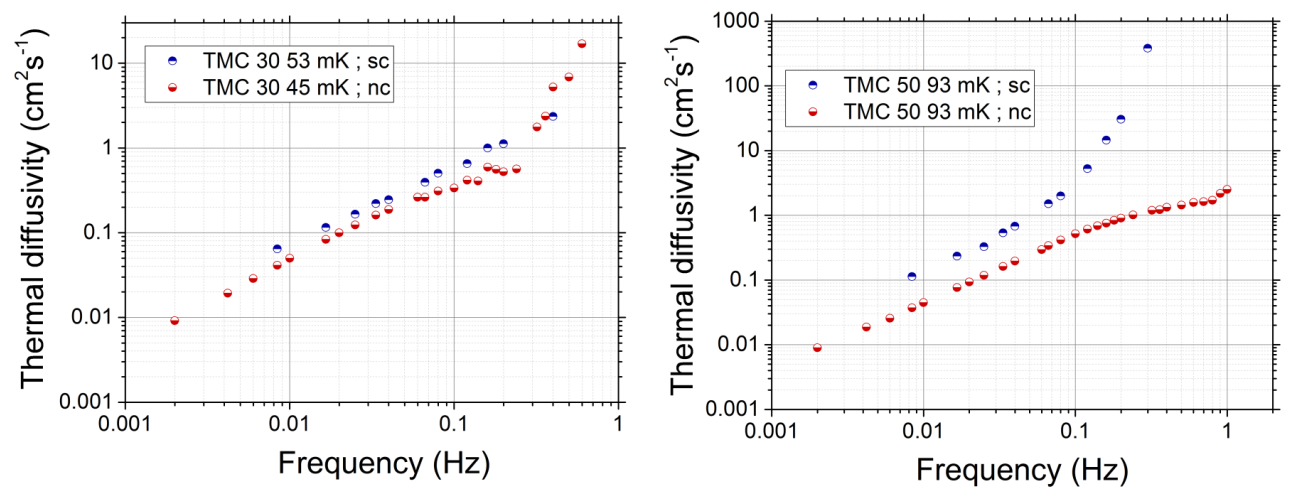

Figure 6.26: Thermal diffusivity of the $\mathrm{Cu}$ - In - $\mathrm{Au}$ - Ti - sapphire sandwich setup as a function of frequency at MC base temperatures of $30 \mathrm{mK}$ (left) and $50 \mathrm{mK}$ (right). The additionally indicated temperature values of $45 \mathrm{mK}$ and $53 \mathrm{mK}$ (left), and $93 \mathrm{mK}$ (right), are mean temperatures between the platform and the stamp 72 .

No inflection points have been observed at these temperatures in the superconducting case, but a plateau can be observed at base MC temperatures of $180 \mathrm{mK}$ and $250 \mathrm{mK}$ (figure 6.27). The diffusivity calculated at the plateau is $a_{\text {sandwich }}^{\mathrm{sc}}=1.2 \pm 0.1 \mathrm{~cm}^{2} / \mathrm{s}$ at an average temperature of $230 \mathrm{mK}$, and $a_{\text {sandwich }}^{\text {sc }}=1.4 \pm 0.1 \mathrm{~cm}^{2} / \mathrm{s}$ at a temperature of $290 \mathrm{mK}[72$. Only four diffusivity measurement values have been reliably obtained in the ultra-low temperature range. 

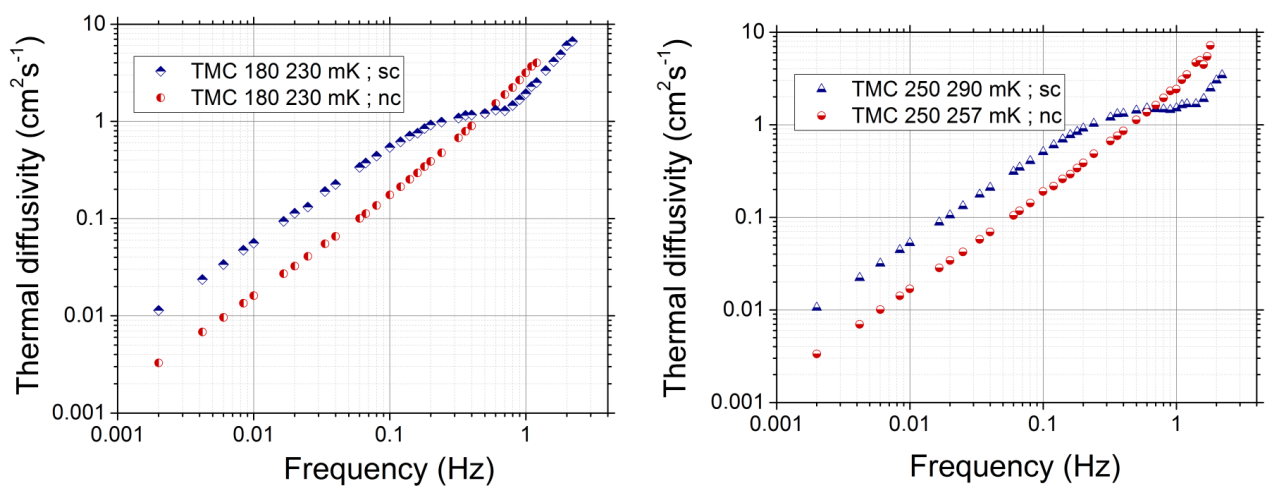

Figure 6.27: Thermal diffusivity of the $\mathrm{Cu}$ - In - $\mathrm{Au}$ - Ti - sapphire sandwich setup as a function of frequency at $\mathrm{MC}$ base temperatures of $180 \mathrm{mK}$ (left) and $230 \mathrm{mK}$ (right). The additionally indicated temperature values of $230 \mathrm{mK}$ (left), $257 \mathrm{mK}$ and $290 \mathrm{mK}$ (right) are mean temperatures between the platform and the stamp 72 .

\subsubsection{Theoretical estimation of the ultra-low temperature thermal diffusivity}

The theoretical estimation of the diffusivity can be done as in section 6.3.3. The thermal conductivity of the copper - indium - gold - titanium - sapphire sandwich is taken from the measurements presented in section 6.2 and calculated according to eq. 6.2. The ultra-low temperature values of specific heat of sapphire, indium and copper were taken from [38]. The contribution of gold and titanium to the heat capacity of the whole sandwich was neglected, because of the very small thickness of these layers. The application of the volume factors $V_{\text {total }} / V_{\text {direct line }}=3.3$ for the copper - indium - sapphire sandwich and 2.7 for the sandwich with titanium and gold, introduced in section 6.3.3, seems to be justified, due to their contribution to the heat capacity influencing transient measurements. The estimated value of the thermal diffusivity with and without the application of a corresponding volume factor, compared to the ultra-low temperature measurement results is presented in figure 6.28. A very good agreement between the measured and predicted values with an application of the volume factor is observed for the sandwich with titanium and gold in both normal and superconducting case. Theoretical values are also plotted for the $\mathrm{Cu}$ - In - sapphire sandwich. The theoretically predicted diffusivity value for the normal conducting case is almost an order of magnitude higher than for the superconducting case, and it is consistent with the experimental data for the sandwich with layers of $\mathrm{Ti}$ and $\mathrm{Au}$. In the superconducting state the $\mathrm{Cu}$ - In - sapphire sandwich should have theoretically a slightly higher value than the sandwich with $\mathrm{Ti}$ and $\mathrm{Au}$, but it was not measured experimentally with frequencies higher than $f_{c 1}$, so a comparison of the measurement results is not possible.

The thermal diffusivity of both setups in the full temperature range of the 


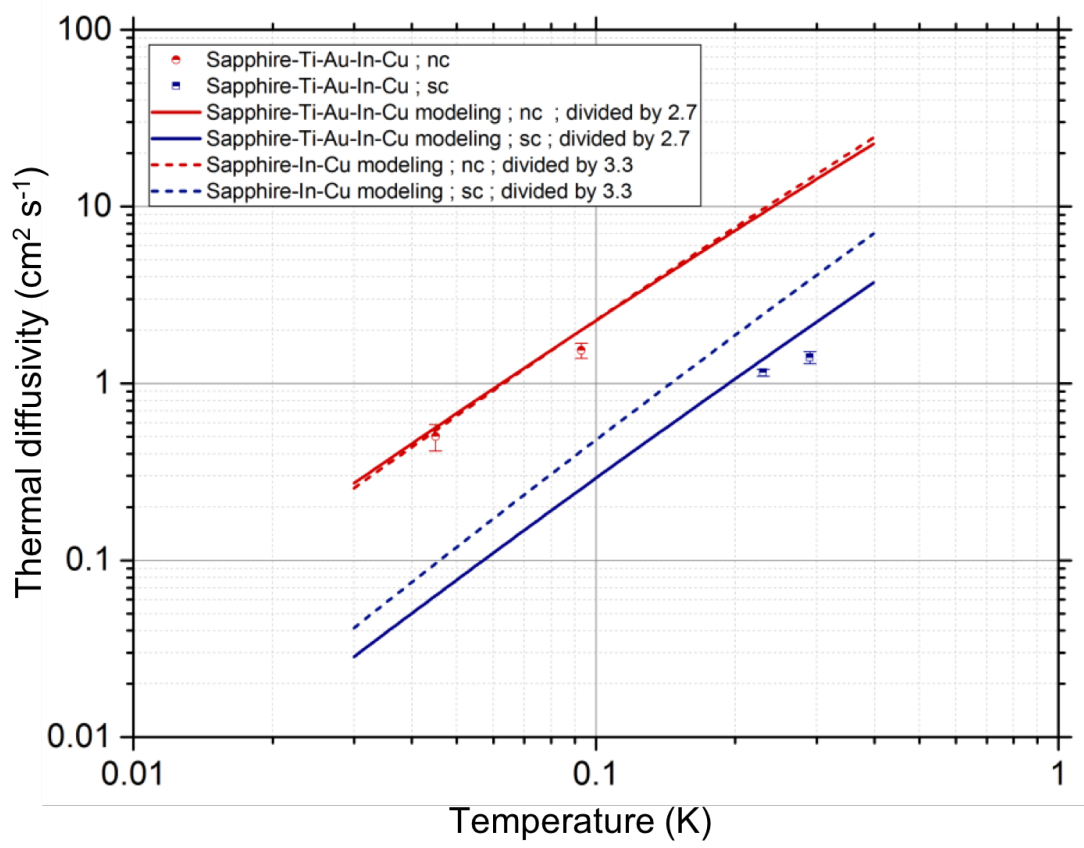

Figure 6.28: Thermal diffusivity of the sandwich setups at ultra-low temperatures obtained from measurements and from the theoretical estimation 72. The theoretical prediction is presented with and without the volume factors included in the calculation. Theoretical values are also plotted for the $\mathrm{Cu}$ - In - sapphire sandwich, which was not tested experimentally.

measurements, from $30 \mathrm{mK}$ to $30 \mathrm{~K}$, is presented in figure 6.29 72. For a better comparison between the two temperature regions the volume reduction factors were not taken into account in the modelling results. Despite a certain gap in the measurements around $1 \mathrm{~K}$, a good coherence of ultra-low and low temperature results is observed. The overall diffusivity of the copper - indium - sapphire setup is higher than of the sandwich with thin layers of titanium and gold, what is probably an effect of the additional interfaces present in the second setup that decrease its thermal conductivity and therefore also the diffusivity. 


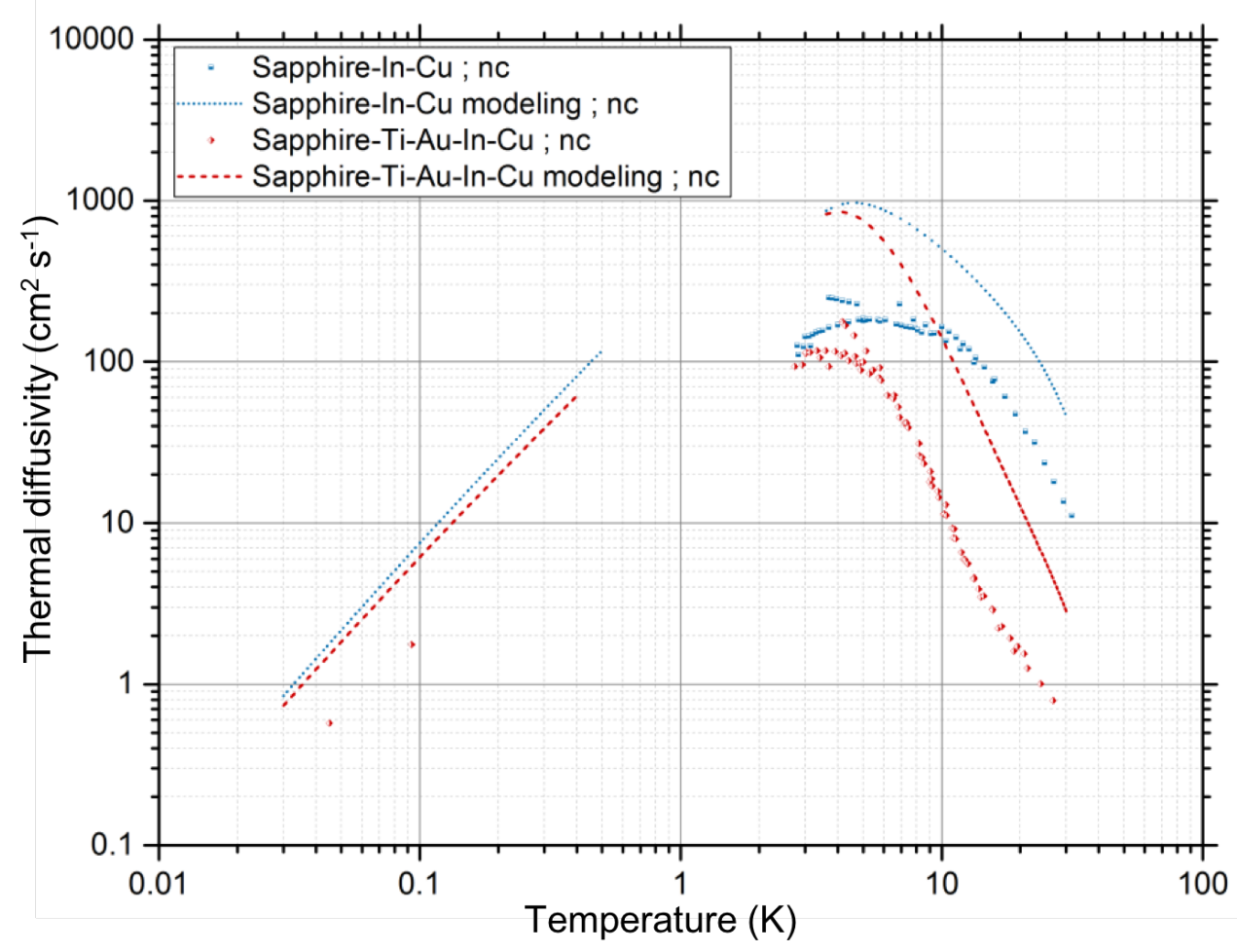

Figure 6.29: Thermal diffusivity of both sandwich setups in a temperature range from $30 \mathrm{mK}$ to $30 \mathrm{~K} 72$. Volume factors were not taken into account in the modelling results.

\subsection{Conclusions drawn from the measurement results}

An unexpected parasitic heat flow via the clamping structure of the first sandwich setup has been observed and quantified. An influence of several factors like ageing, alloying, formation of intermetallic compounds and oxidation of the surface on the interface thermal resistivity and on the total thermal resistance of the sandwich has been explained. A mathematical model has been created corresponding to all measured configurations of the setup.

The thermal diffusivity of both sandwich setups has been measured and modelled. The measurement results of the first sandwich setup correspond to the model with a volume factor included. The measured thermal diffusivity of the second sandwich is lower than predicted by the model.

A clear conclusion can be drawn that the presence of the thin layers of titanium and gold significantly decreases not only the thermal conductivity, but also the thermal diffusivity of the sandwich. Therefore, the deposition of these layers should be avoided on the bottom surfaces of the electrodes, which are responsible for the thermal anchoring of the electrodes to the cooling source in the AEgIS experiment. 
The most important outcome of the measurement results is that when indium is in the normal conducting state, which is the case in the AEgIS experiment, the clamping structure can be removed without a significant change in the quality of the thermal connection to the mixing chamber. Thanks to this information, a modification of the electrode is possible to improve its thermal performance. Because of the $1 \mathrm{~T}$ magnetic field surrounding the electrodes, the main focus of the next chapter will be the analysis of the thermal performance of the electrode in the normal conducting state. 


\section{Chapter 7}

\section{Ultra-cold electrode design and thermal performance}

The ultra-cold electrodes, which the Penning trap in the AEgIS experiment will consist of, should fulfill a series of very strict requirements, summarised in chapter 1. After a series of preliminary measurements, the first design of the electrode was made in the Cryolab by G. Burghart and is presented in figure 7.1. Unfortunately, its thermal performance was a few times lower than expected [7]. Several factors that might have influenced it are described below.

\subsection{Stress analysis and the quality of the indium bond in the old electrode}

The complicated shape of the electrode and its small dimensions make the phonon path in the dielectric long and narrow, with the smallest cross-section next to the cylindrical hole separating sectors of about $12 \mathrm{~mm}^{2}$ (see figure 7.1. . The way the electrode was mounted, i.e. supported at the bottom and pressed with the bolts on both sides (see picture 7.1), caused bending of its delicate structure and introduced mechanical stresses. The plot of the equivalent (von Mises) stress is presented in figure 7.2. The highest value of the stresses occurred in the place of the smallest cross-section, where it acts like a "dislocation" of the sapphire lattice and additionally disturbs heat transfer (see chapter 22). The smallest cross-section became a significant thermal bottle-neck.

The bending of the electrode influenced also the quality of the indium bond at the bottom. As presented in figure 7.3 , the pressure distribution was not uniform and as a result the correct indium bond, described sometimes as a "cold weld" [5, 6] can be created only on a small fraction of the whole surface, corresponding to the spots with the highest pressure, as indicated by the pressure distribution obtained from the ANSYS $^{\circledR}$ simulation (compare figures 7.3 and 7.4 . After dismounting of the electrode setup the indium cold 
weld was established only at the spots of the highest force, at similar locations as predicted by the numerical simulation.

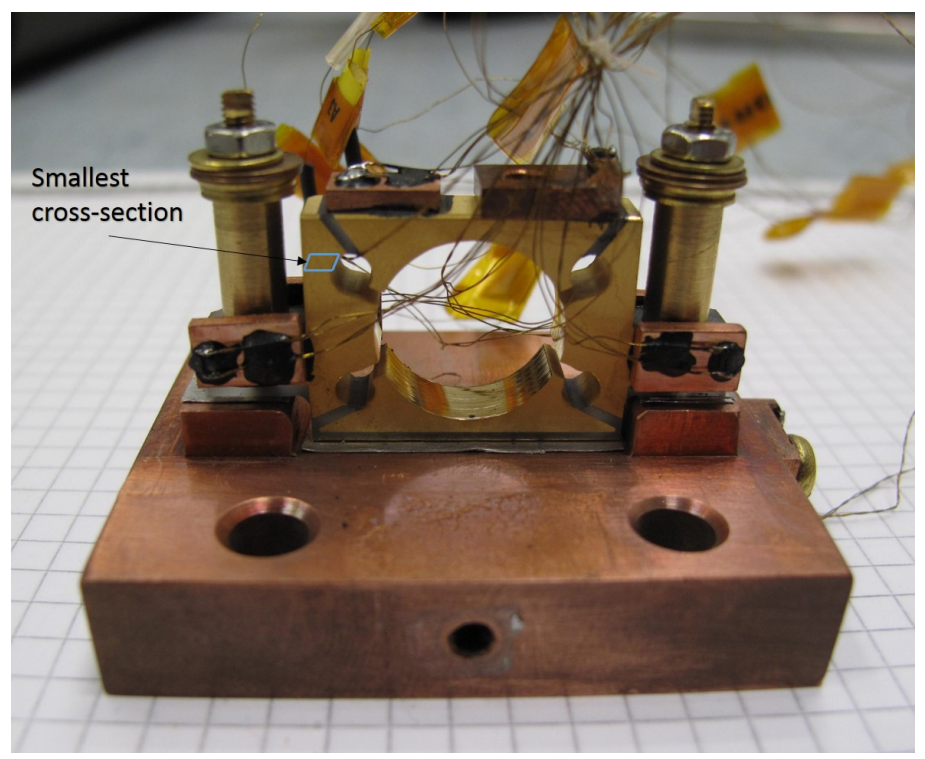

Figure 7.1: The electrode designed by G. Burghart fully instrumented for the measurements in the Cryolab DR. Picture taken by H. Derking after dismounting the electrode with its thermalisation platform from the lid of the mixing chamber.

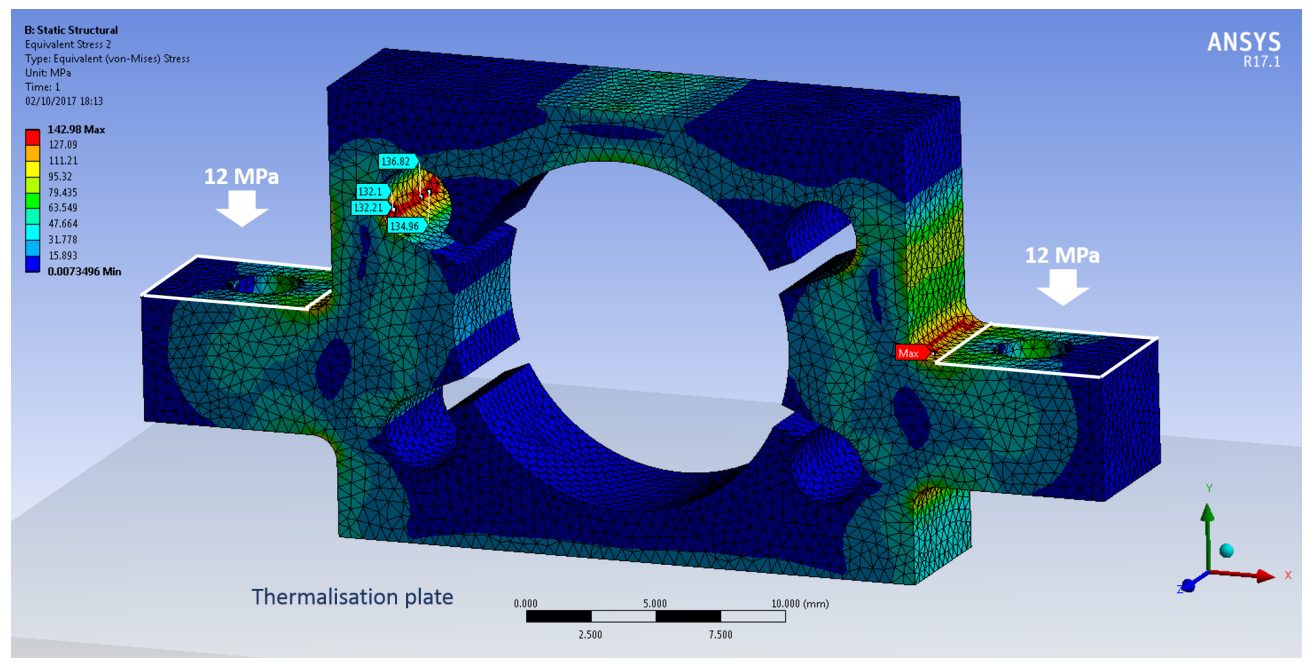

Figure 7.2: Finite Element Method simulation of mechanical stresses in the electrode designed by G. Burghart. Applied pressure of $12 \mathrm{MPa}$ (corresponding to the force generated by the bolts) on the sides of the electrode results in around $7 \mathrm{MPa}$ at the bottom surface pressed to the thermalisation plate. The electrode mounted in this way has a tendency to bend and the highest stresses occur in the place of the smallest cross-section (red colour), along the main heat transfer path. 


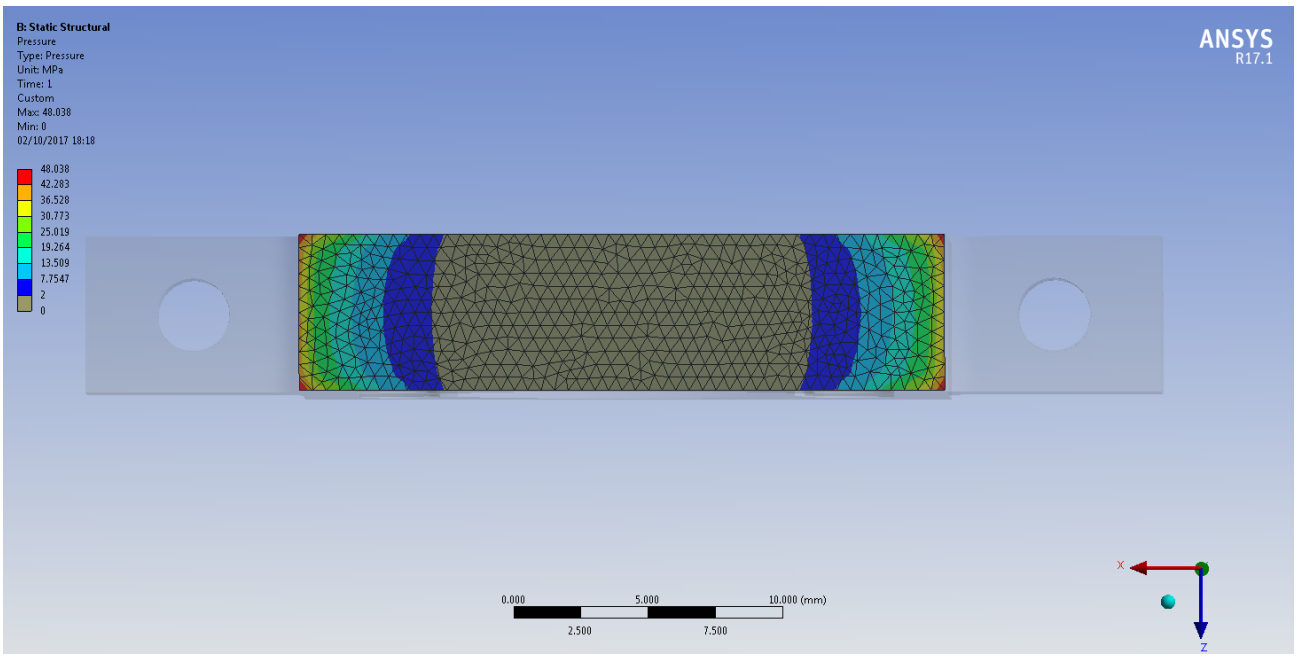

Figure 7.3: Plot of the pressure distribution at the bottom of the old electrode designed by G. Burghart, loaded with a pressure of $12 \mathrm{MPa}$ on each side, obtained from the Finite Element Method simulation. Grey colour indicates the area where the pressure in indium is lower than $2 \mathrm{MPa}$, and therefore insufficient for the formation of a solid indium bonding [6]. The stress concentration up to $40 \mathrm{MPa}$ in the corners is a typical result of a numerical simulation of two compressed rigid bodies. In reality the distribution will be a bit more uniform, because of a layer of plasticised indium.

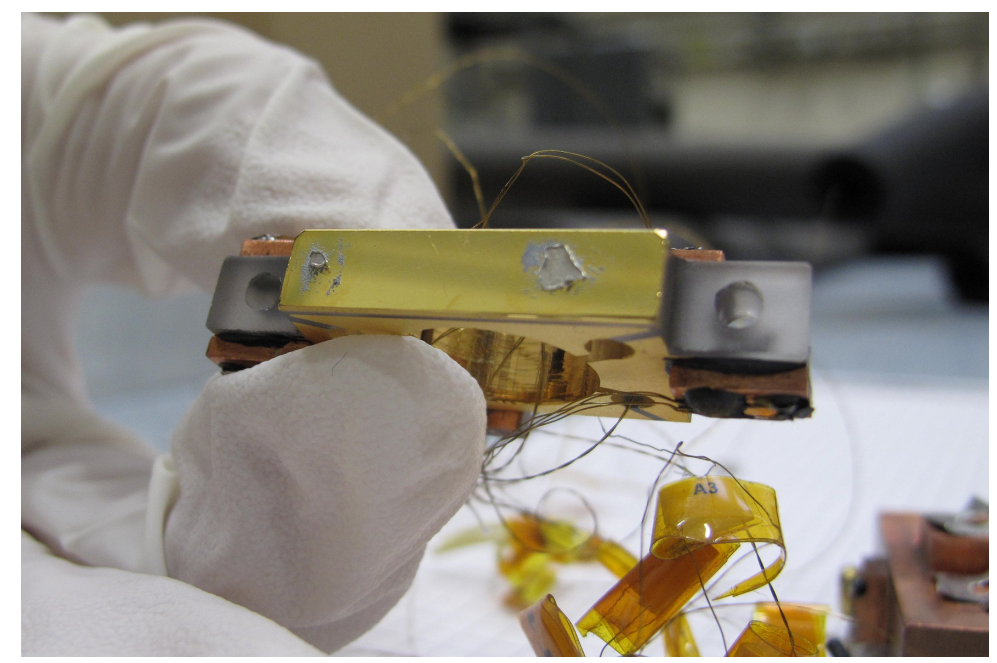

Figure 7.4: Traces of the indium bond at the bottom of the electrode designed by G. Burghart. Their position corresponds to the pressure distribution in figure 7.3 Picture taken by $\mathrm{H}$. Derking.

A more uniform way of pressing the electrode provided by another type of clamping could result in the formation of the cold weld on a larger part of the bottom surface of the electrode, and therefore highly increase the effective 
contact area and improve the heat transfer.

As concluded in chapter 6 , in the presence of an external magnetic field, the clamping structure can be removed without a significant increase of the copper - indium - sapphire thermal boundary resistance. Thanks to the possibility of the removal of the clamping structure, one can not only save some precious space in the cold bore of the AEgIS experiment, but also attach the electrodes in a different way, providing a much more uniform pressure distribution at the bottom of the electrodes during the clamping procedure. The removal of the clamping structure eliminates also the mechanical stresses in the critical cross-section of electrode, what should improve the heat transport across the sapphire itself.

\subsection{Thin layers and their influence on the thermal performance of the electrode}

The thermalisation platform on the mixing chamber of the dilution refrigerator responsible for providing the cooling power to the ultra-cold electrodes in the AEgIS experiment will probably be made of OFHC copper. Therefore a layer of indium foil is necessary to provide proper mechanical attachment and thermal contact via an indium bond between the electrodes and the platform. The vapour deposition method was proved to be a robust way of bonding the dielectric sapphire with metallic layers [4].

The thermal conductivity of a sapphire - indium - copper sandwich was demonstrated to be approx. $27 \mathrm{~cm}^{2} \mathrm{~K}^{4} / \mathrm{W}$ at $100 \mathrm{mK}$ on the warmer side of the interface (fig. 6.5). A quick estimation of the amount of heat transferred across the interface between the electrode and the thermalisation plate, assuming that the temperature of the plate is at approximately $80 \mathrm{mK}$, and the electrode at $100 \mathrm{mK}$, can be evaluated to be $0.5 \mu \mathrm{W}$ per $\mathrm{cm}^{2}$ of area:

$$
\begin{aligned}
\dot{Q}= & \frac{A}{4 \cdot 27 \mathrm{~cm}^{2} \mathrm{~K}^{4} / \mathrm{W}}\left((0.1 K)^{4}-(0.08 K)^{4}\right) \approx \\
& \approx 0.5 \cdot 10^{-6} \frac{\mathrm{W}}{\mathrm{cm}^{2}} \cdot A=0.5 A \mu \mathrm{W},
\end{aligned}
$$

where $A$ is in $\mathrm{cm}^{2}$. Thus a surface of around $2 \mathrm{~cm}^{2}$ should be sufficient to provide at least $1 \mu \mathrm{W}$ of heat flow extracted by the cold source. More heat can be extracted if the mixing chamber and the thermalisation plate are colder than $80 \mathrm{mK}$.

As concluded in chapter 6 the presence of the thin layers of titanium and gold forming the four sectors on the electrode should be avoided on the bottom surface of the sapphire, as it significantly decreases the rate of heat transfer. The deposition of four sectors on the complicated shape of the electrode has to be done with a complex mask, to avoid merging of the sectors. It is also 
important to keep the lowest sector of the electrode electrically separated from the bottom surface, which is attached to the metallic platform for thermalisation. Therefore, the masks for deposition of titanium-gold and later indium should be designed and manufactured with very high precision. The sputtering of titanium and gold should be done first and the vapour deposition of indium afterwards, because of the lower temperature of vapour deposition of indium, compared to the sputtering of gold.

\subsection{New design of the electrode and its manufacturing}

Having gained experience with the first design, a series of improvements was implemented in the new design of the electrode, incorporating the results of the studied sandwich configurations, in order to increase its thermal performance both in steady state and transient conditions. The recommendations for the new design, which were formulated as a conclusion from the analysis are the following:

- increase the size of the smallest cross-section, not to create a geometrical thermal bottle-neck along the thermal pathway in the sapphire,

- avoid the stress concentration at the smallest cross-section by modifying the shape of the electrode and the way it is mounted,

- obtain a better quality cold weld at a larger part of the bottom surface of the electrode by modifying the clamping structure;

- avoid the presence of thin layers of titanium and gold on the bottom of the electrode.

The most important result of the sandwich setup study is that the application of force to this type of pressed contact is not necessary for proper heat transfer in the presence of magnetic field, causing indium to be normal conducting. Therefore, the space previously occupied by the clamping structure can now be used for significant modification of the shape of the electrode. The new design of the electrode has a wider top part to increase the cross-section size of the critical heat path. Thanks to the fact that the electrode does not have to be pressed during the cooldown and operation, the holes for the bolts can be eliminated, and despite a smaller total external dimension, the bottom surface can be increased. Omitting the clamping structure, that compresses the electrode in the experiment, excludes the mechanical stresses in the material, especially around the critical cross-section (compare figure 7.2 and 7.5) that could limit heat transfer after the cool-down, as explained in chapter 2 , Removal of the clamping structure allows us also to press the electrode not only from the top, but also in the middle, what makes the pressure distribution on 
the bottom much more uniform, the stresses in the sapphire smaller, and the chances for the cold weld formation much higher.

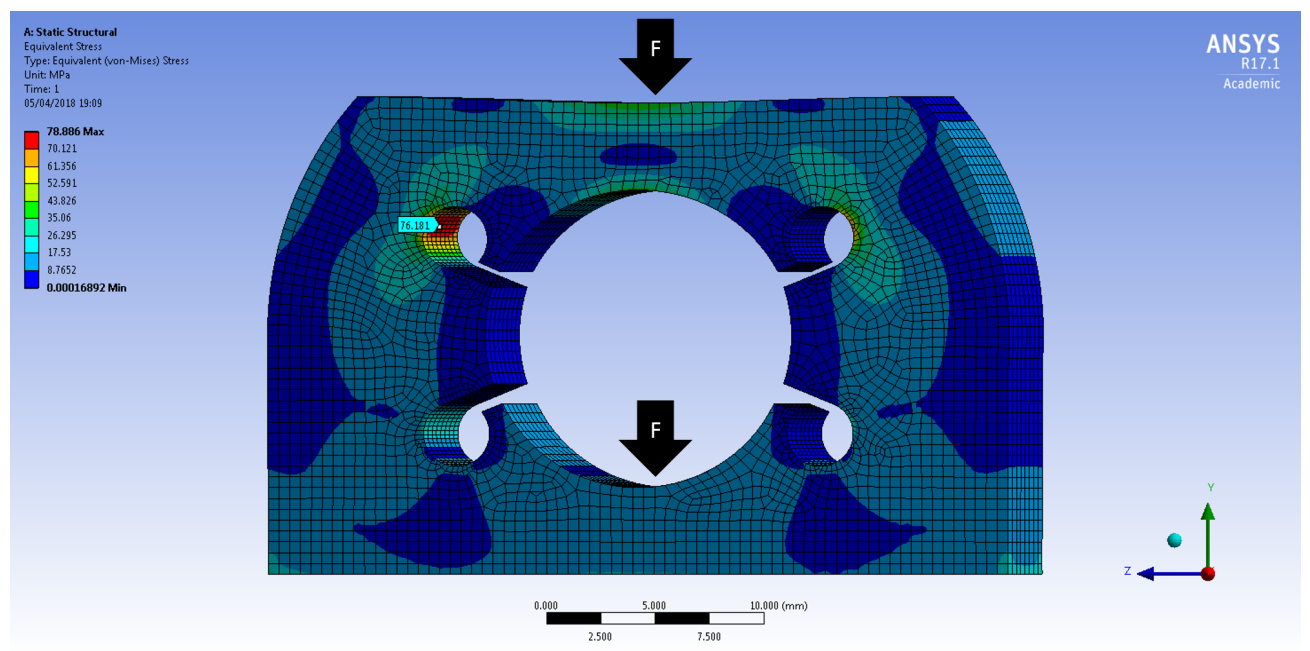

Figure 7.5: The 3D distribution of von Mises stresses in the new electrode design. The structure was loaded with a force $\mathrm{F}=471 \mathrm{~N}$ on top of the electrode and another $471 \mathrm{~N}$ in the middle of the electrode, which results in a pressure on the bottom of the electrode of approx. 4.7 MPa. Such a pressure is sufficient to cause creeping of indium and to form a proper indium bond. The applied forces correspond to the way the electrode will be later fixed on the platform.

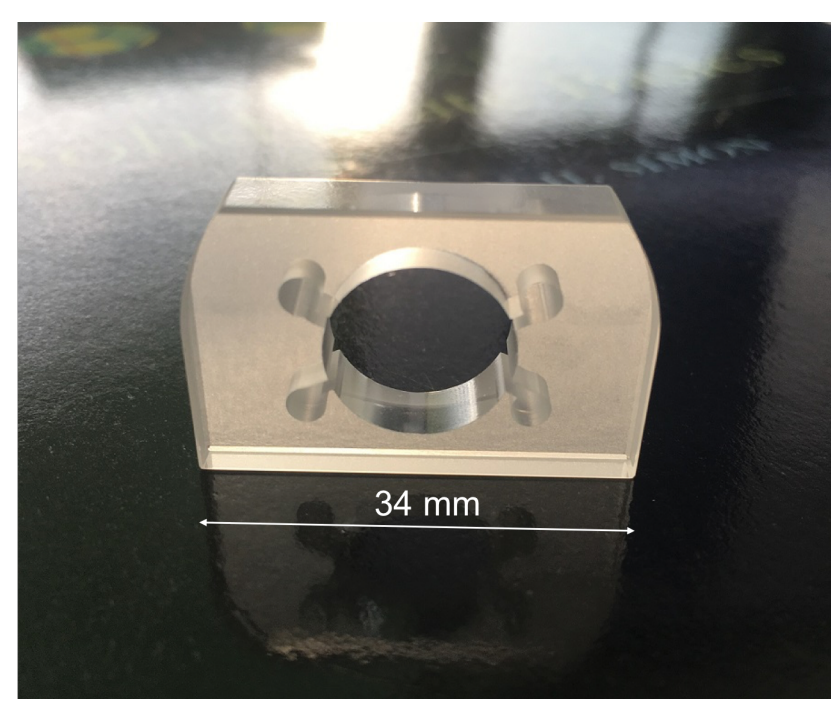

Figure 7.6: The sapphire base for the new electrode manufactured by Dr. Sztatecsny GmbH, as delivered. One can see the polished cylindrical surface in the centre.

The electrode according to the new design was manufactured by Dr. Sztatecsny $\mathrm{GmbH}$. The sapphire, just after arrival from the manufacturer, is 


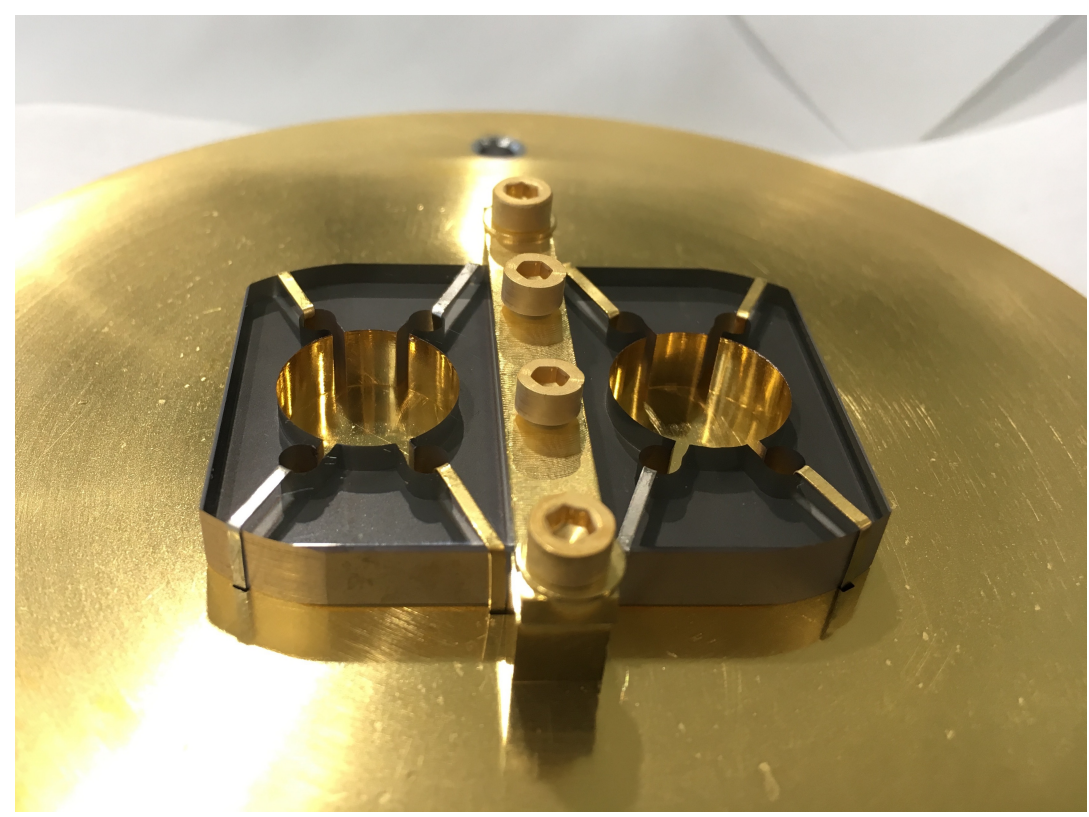

Figure 7.7: Two electrodes in a mask for gold deposition. Titanium and gold were already sputtered on one face and the circumference surfaces. The electrodes are ready for the deposition on the second face. The dark grey colour visible across the sapphire comes from the $45 \mathrm{~nm}$ layer of titanium on the opposite face of the electrode, which was sputtered first. Thin steel stripes keeping the electrode on the plate are responsible for providing a sufficient separation of the sectors. The bottom surfaces of the electrodes, where later on the indium deposition will follow, are protected by the central bar.

presented in figure 7.6 and the corresponding technical drawing with all the dimensions and tolerances can be found in the appendix.

The four sectors of the electrodes were created on a sapphire base with a magnetron sputtering technique. Because of the poor adhesion of gold on sapphire, it is necessary to add a $45 \mathrm{~nm}$ layer of titanium below the $520 \mathrm{~nm}$ thick layer of gold. Electrodes in a mask for gold deposition with one side already covered with titanium and gold, and the second side ready for deposition, are presented in figure 7.7. The separation of the four sectors is obtained thanks to a mask of $1 \mathrm{~mm}$ thick stainless steel strips. It is very important to keep the bottom surface of the electrode electrically separated from the side sectors, which is established by the central holder of the deposition mask.

Afterwards, a $600 \mathrm{~nm}$ layer of indium was vapour deposited on the bottom surfaces of both electrodes without any under-layer. The mask for indium deposition, designed in a way to make sure that the bottom surface would not be electrically connected with the side sectors, is presented in figure 7.8 . While placing the electrodes in the mask for indium deposition, one has to pay special attention trying not to scratch the golden sectors.

After the deposition of the electrode sectors and the bottom indium inter- 


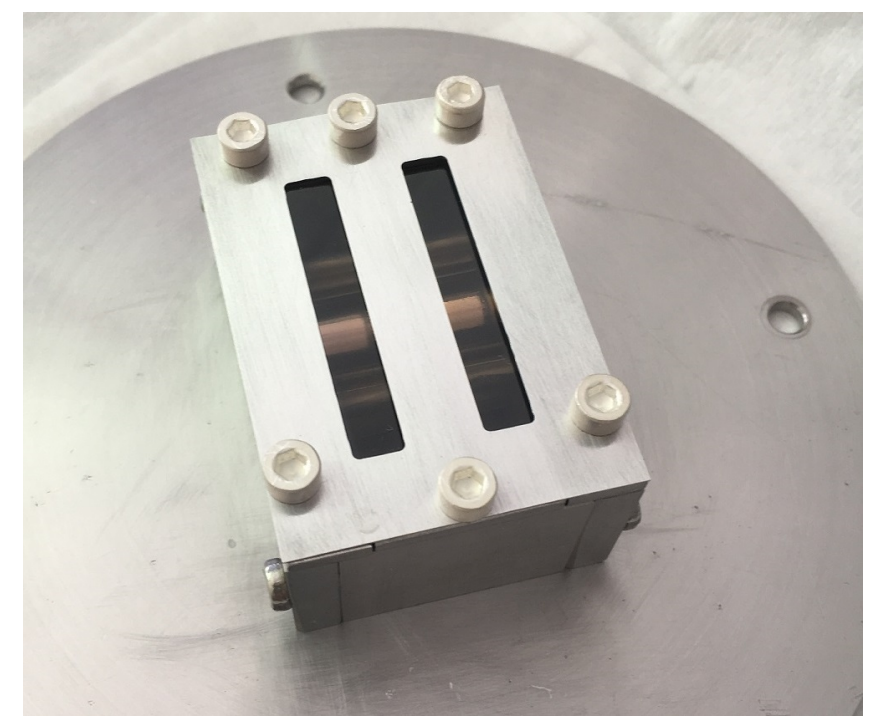

Figure 7.8: Electrodes in the mask for indium vapour deposition. Only the bottom surfaces of the electrodes are visible and are exposed to the vapour deposition process.

face, one of the electrodes was placed on a copper platform with 125 um thick indium foil in between, to install and measure its properties in the DR. It is essential to remove the layers of oxides from both copper and indium to make sure they are not disturbing the formation of the cold weld. To remove the oxide, the surfaces could be scratched or treated with mild $\mathrm{HCl}$ solution [6]. In the case of copper, mild scratching with Scotch-Brite was sufficient, whereas the indium was etched with $\mathrm{HCl}$ and rinsed with demineralised water and isopropanol. The electrode was pressed with a clamping sturcture designed in a way to provide a uniform pressure distribution in the layer of indium at the bottom of the electrode. The four M3 bolts, presented in figure 7.9 on the left, were tightened with a very low torque of $10 \mathrm{cNm}$ each. Such torque generates a compression force of around $235 \mathrm{~N}$ in each screw, which results in a pressure of $4.7 \mathrm{MPa}$ at the bottom of the electrode. The pressure distribution at the bottom of the electrode was estimated with the Finite Element Method, and the result of the simulation is shown in figure 7.10. One can see that the obtained pressure distribution is way more uniform than for the old design of the electrode (compare figures 7.3 and 7.10 . On the whole bottom surface the pressure is higher than 3.7 MPa, which should be sufficient for the formation of the cold weld [5,6]. To check the quality and robustness of the obtained indium bonding, the electrode was kept hanging on a horizontal rod wrapped in a soft piece of cloth for 24 hours as shown in figure 7.9 on the right. No sign of any degradation of the bond was observed. 

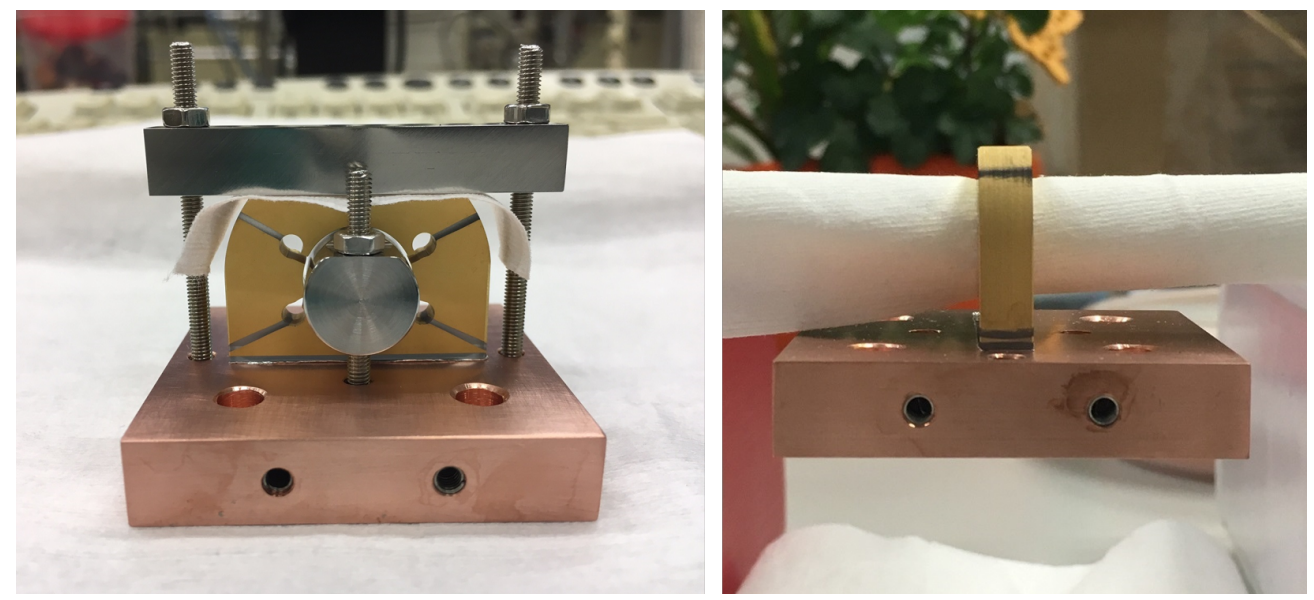

Figure 7.9: Left: One of the electrodes pressed with four M3 screws. Each screw was tightened with a torque of $10 \mathrm{cNm}$, which gives a force of around $235 \mathrm{~N}$ per screw, and $4.7 \mathrm{MPa}$ pressure on the indium foil below the electrode. Right: Test of the indium bonding. The cold weld withstood $24 \mathrm{~h}$ of the platform's weight hanging below the electrode without any sign of degradation of the bond.

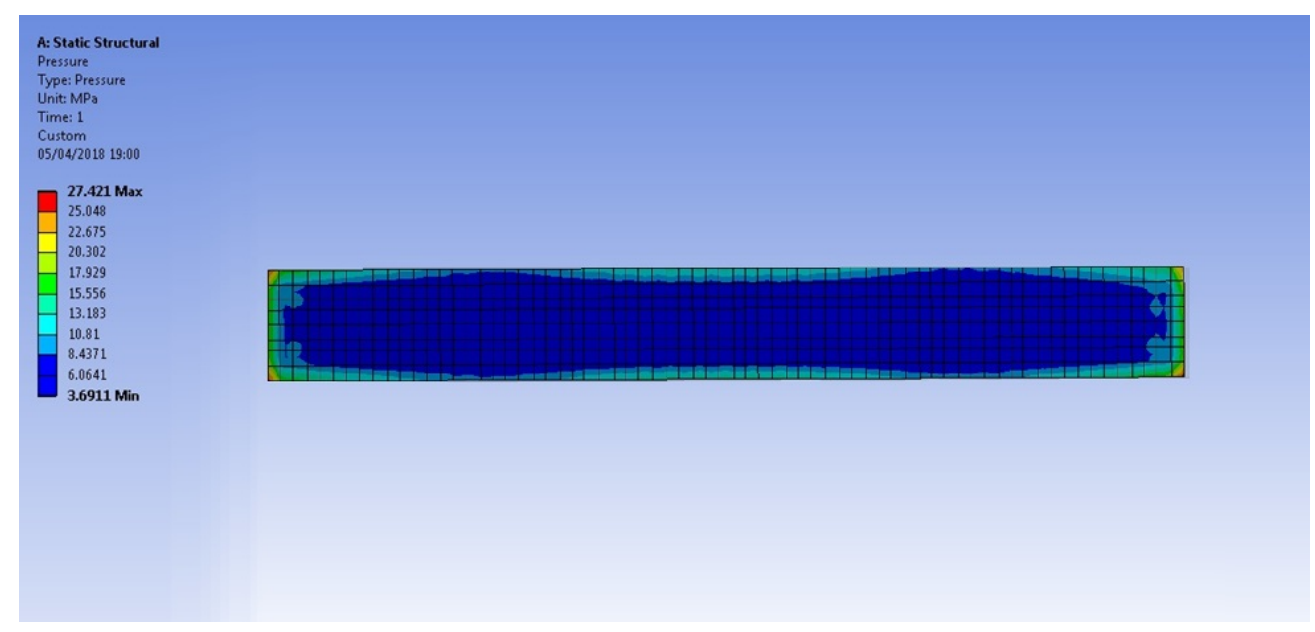

Figure 7.10: Simulation of the pressure distribution on the bottom of the new electrode, compressed as presented in figure 7.9 (left) with a torque of $10 \mathrm{cNm}$ in each screw. The pressure distribution is very uniform, with the lowest value of approx. $3.7 \mathrm{MPa}$ - exceeding the yield limit of indium. For sufficiently clean indium, such a uniform distribution highly increases the chance of a uniform cold weld at the bottom surface of the electrode. Simulation with ANSYS ${ }^{\circledR}$. Stress concentration up to $27 \mathrm{MPa}$ visible in the corners is a typical result of a numerical simulation and presents no ground for a concern.

\subsection{Ultra-low temperature measurements}

The same temperature sensors and heater, that were previously used on sandwich setups, have been glued to the electrode with Stycast ${ }^{\circledR}$. The electrode, 
cold welded to the platform and mounted on the lid of Cryolab DR mixing chamber, is presented in figure 7.11 on the left. The exact position of sensors is schematically marked in the same figure on the right.
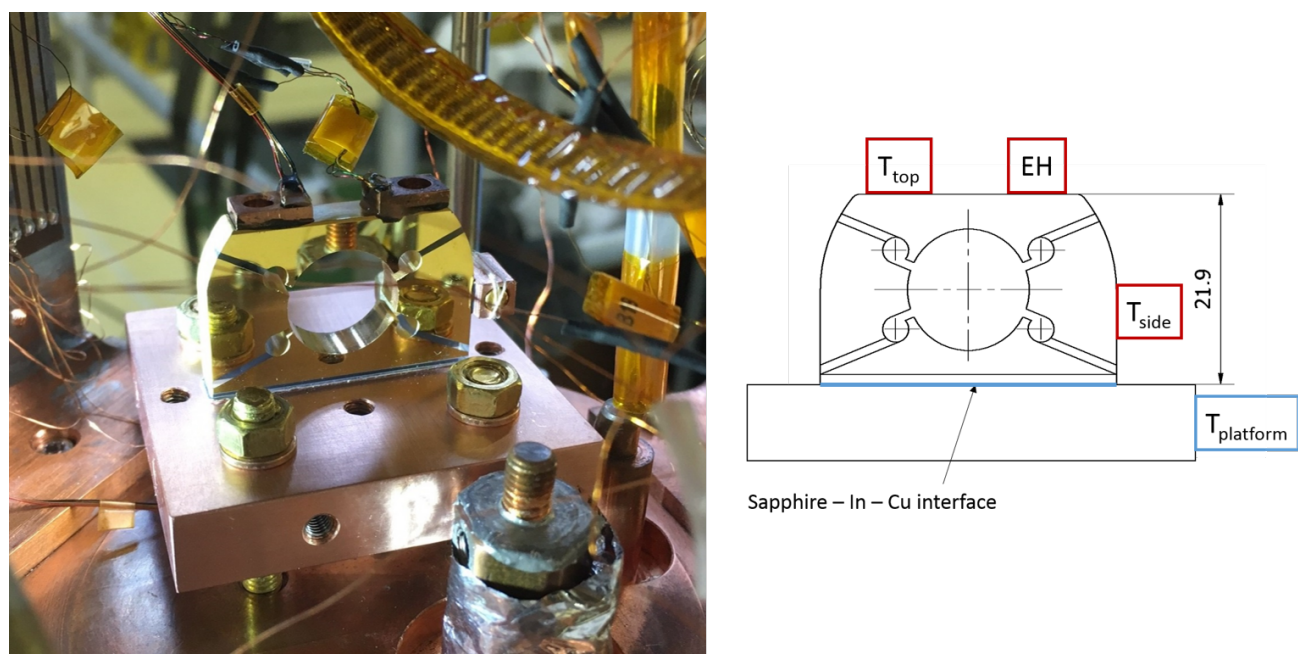

Sapphire - In - Cu interface

Figure 7.11: Left: picture of the new electrode with the top and side sensors and the heater glued, mounted on the lid of the mixing chamber. Right: a part of the technical drawing of the electrode with the positions of the electric heater and sensors marked.

During the measurements the heat was supplied by the electric heater $(\mathrm{EH})$ on the top sector of the electrode and the temperatures on the top and side sectors were measured. The temperature range of the measurements corresponds to the requirements of the application and to the research done on the previous version of the electrode. The measurements were done both with and without magnetic field, at $30 \mathrm{mK}, 50 \mathrm{mK}$ and $70 \mathrm{mK}$ base temperatures of the platform in steady state and transient conditions. A few transient measurement points were taken also at $17 \mathrm{mK}$ base temperature of the mixing chamber.

\subsubsection{Steady state measurement results}

The most important question that should be answered in steady state measurements is, what amount of heat can be extracted from the electrode, keeping its temperature below $100 \mathrm{mK}$. Measurements on the old electrode showed that at a base temperature of $50 \mathrm{mK}$ the temperature of the side sensor reaches 100 $\mathrm{mK}$ for a heat load of $0.20 \mu \mathrm{W}[7]$, which is around 4 times less than expected from the preliminary measurements. The side electrode sensor was chosen as the representative one for the behaviour of the electrode, because it reflects better the real conditions of the electrodes in the AEgIS experiment where the heat will be distributed more uniformly than just in the top sector. A full plot of the measured side temperature as a function of the applied heat load, for all base temperatures, is presented in figure 7.12 . The lower the temperature of the 
platform, the more heat can be extracted from the electrode keeping it below $100 \mathrm{mK}$. The amount of heat transferred via the new design of the electrode for the same conditions as in [7], i.e. base temperature of $50 \mathrm{mK}$ and probing the electrode temperature with the side sensor, equals to $1.12 \mu \mathrm{W}$, which is 5.6 times more than before. The more than 5 times improvement, comes from a series of modifications, and can be considered a success.

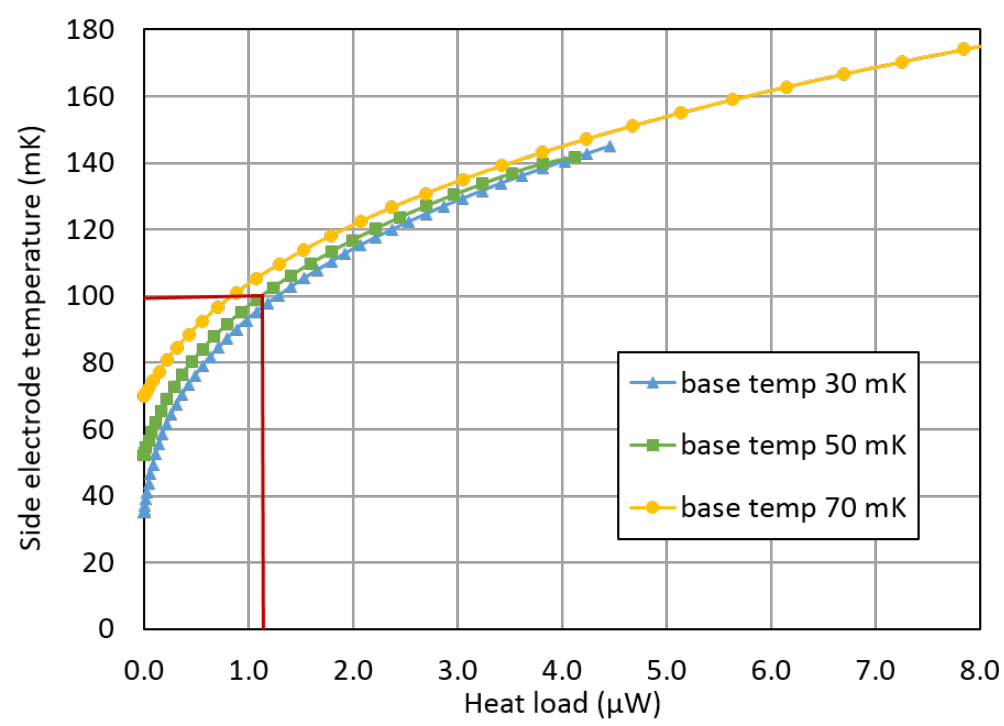

Figure 7.12: The temperature of the side of the electrode as a function of the applied heat load for different base temperatures. The amount of heat transferred while keeping the electrode at $100 \mathrm{mK}$ equals $1.12 \mu \mathrm{W}$. The lower the base temperature, the higher the amount of heat transferred, while keeping the electrode at $100 \mathrm{mK}$.

The Kapitza-like thermal resistivity of the electrode - platform, i.e sapphire - indium - copper connection, calculated with the temperature of the side sensor corresponds to the resistivity value of the sandwich setup measured before. In the temperature range of $40 \mathrm{mK}-180 \mathrm{mK}$, the determined resistivity has a value of $22 \frac{\mathrm{cm}^{2} \mathrm{~K}^{4}}{\mathrm{~W}}-56 \frac{\mathrm{cm}^{2} \mathrm{~K}^{4}}{\mathrm{~W}}$, as presented in figure 7.13 . The old electrode did not reach a temperature low enough for a direct comparison, but in a temperature range of $80 \mathrm{mK}-200 \mathrm{mK}$, the thermal resistivity had a value of $210 \frac{\mathrm{cm}^{2} \mathrm{~K}^{4}}{\mathrm{~W}}-260 \frac{\mathrm{cm}^{2} \mathrm{~K}^{4}}{\mathrm{~W}}$ respectively 7 , which is several times higher than for the new design. In figure 7.13 the thermal resistivity of the sandwich sapphire - In $\mathrm{nc}$ - $\mathrm{Cu}$ setup is given as a comparison. At the lowest temperatures the thermal resistivity is almost the same for both setups: the electrode and the sandwich. The values diverge with an increase of temperature and heat load. The difference must be a result of a large amount of sapphire in the electrode. The effect of an increase of the thermal resistivity with an increase of the sapphire thickness has been observed in figure 6.5. 


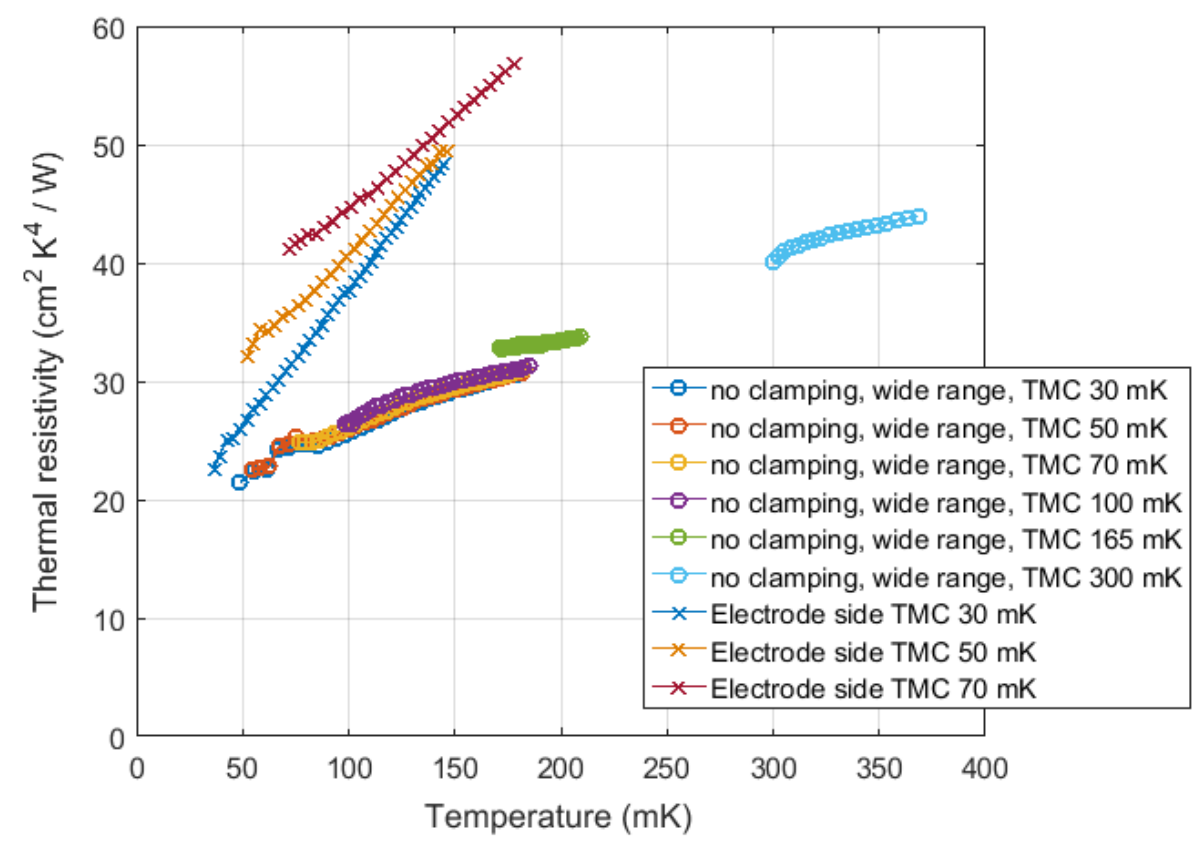

Figure 7.13: Thermal resistivity of the electrode - platform connection with indium in normal conducting state for three base temperatures. The resistivity values are calculated for the side electrode sensor. As a reference, previous results of the sapphire - In nc - $\mathrm{Cu}$ sandwich are shown (see figure 6.5.

\subsubsection{Response to transient heat loads}

If the main constraint for the heat flow in the electrode were the Kapitza-like interface resistance to the platform on which the electrode is mounted, the system could be simplified to a lumped one, consisting of a mass of certain heat capacity $C$ representing mainly the sapphire, as the contribution of thin layers to the heat capacity is negligible, and the thermal resistance $R$ of the electrode - platform interface. Whether that is the case, can be judged calculating the Biot number. The Biot number is the ratio of the heat transfer across the external surface of the body to the internal heat transport $\mathrm{Bi}=\frac{l_{\mathrm{Bi}} h_{\mathrm{Bi}}}{\lambda_{\mathrm{Bi}}}$, where $l_{\mathrm{Bi}}$ is a characteristic dimension of the body, $h_{\mathrm{Bi}}$ is the heat transfer coefficient across its external surfaces, and $\lambda_{\mathrm{Bi}}$ is the thermal conductivity of the body. It indicates whether a body cools down or warms up uniformly or if the internal temperature gradient is significant. The number lower than 0.1 informs that the system can be successfully modelled as a lumped one. For the heat transfer in the electrode the characteristic dimension is its height $l_{\mathrm{Bi}}=21.9 \mathrm{~mm}$. The ultra-low temperature thermal conductivity of sapphire from section 5.2 at $100 \mathrm{mK}$ equals $\lambda_{\mathrm{Bi}}=2.3 \cdot 10^{-3} \mathrm{~W} /(\mathrm{m} \cdot \mathrm{K})$. The heat transfer coefficient between the electrode and the platform read out from figure 6.5 for an average 
temperature of the interface $T_{\mathrm{av}}=90 \mathrm{mK}$, as used in equation 7.1. equals $h_{\mathrm{Bi}}=\frac{T_{a v}^{3}}{\kappa}=\frac{(0.09 \mathrm{~K})^{3}}{27 \mathrm{~cm}^{2} \mathrm{~K}^{4} / \mathrm{W}}=0.27 \mathrm{~W} /\left(\mathrm{m}^{2} \mathrm{~K}\right)$. Thus, the Biot number for the electrode kept at around $100 \mathrm{mK}$ equals $\mathrm{Bi}=2.57$, indicating that the temperature gradient across the electrode is significant, and the system cannot be modelled as a lumped one.

The values of heat capacity $\mathrm{C}$ and resistance $\mathrm{R}$ can be used to estimate the time constant describing the transient behaviour of the system by analogy to its electric equivalent [113]. The time constant is usually called $R C$ or $\tau$ and has a unit of seconds. It can be obtained from measurements by finding a time (from the beginning of step like heating or cooling) after which the temperature change equals to $63 \%$ of $\Delta T_{\max }$, because $e^{-t / \tau}=e^{-1}=0.37$.

The time constant of the electrode can be estimated. The volume of sapphire in the electrode equals to $3.066 \mathrm{~cm}^{2}$ and with a density of $4 \mathrm{~g} / \mathrm{cm}^{3}$, it gives a mass of $12.26 \mathrm{~g}$. The ultra-low temperature specific heat of sapphire at $100 \mathrm{mK}$ is evaluated to be around $2.83 \cdot 10^{-10} \mathrm{~J} /(\mathrm{g} \mathrm{K})$ (extrapolated towards low temperatures from $\left[12\right.$ ), so $C_{\text {electrode }}=3.47 \cdot 10^{-9} \mathrm{~J} / \mathrm{K}$, which is an extremely small value. The characteristic resistance of the sapphire - indium - copper interface at an average temperature of $90 \mathrm{mK}$ equals $27 \mathrm{~cm}^{2} \mathrm{~K}^{4} / \mathrm{W}$, and the bottom area of the electrode $A_{\text {interface }}=1.972 \mathrm{~cm}^{2}$, giving $R_{\text {interface }}=1.88 \cdot 10^{4} \mathrm{~K} / \mathrm{W}$. This results in a time constant $\tau=6.5 \cdot 10^{-5} \mathrm{~s}$, which is very short. Since the ultra-low temperature dependence of the specific heat of sapphire is $c_{p} \propto T^{3}$ and the interface resistance $R \propto T^{-3}$, the $R C$ value should be temperature independent, if the internal temperature gradients were insignificant. The internal resistance of the sapphire should be included in the estimation because of the relatively high Biot number indicating a significant resistance from the sapphire itself. The smallest cross-sectional area of the heat path in sapphire electrode equals $A_{\min }=0.348 \mathrm{~cm}^{2}$ and the length of the heat path in the sapphire electrode is approximately equal to the height of the electrode $l=2.19 \mathrm{~cm}$. Taking the sapphire conductivity of $2.3 \cdot 10^{-5} \mathrm{~W} /(\mathrm{cm} \mathrm{K})$ at $100 \mathrm{mK}$ (as for the Biot number estimation), the corresponding resistance of the electrode equals $R_{\text {sapphire }}=\frac{2.19 \mathrm{~cm}}{2.3 \cdot 10^{-5} \mathrm{~W} /(\mathrm{cm} \mathrm{K}) \cdot 0.348 \mathrm{~cm}^{2}}=2.74 \cdot 10^{5} \mathrm{~K} / \mathrm{W}$, which is indeed much more than the resistance of the interface, and the total time constant of the system equals around $\tau \approx 1 \mathrm{~ms}$. Such would be the time constant corresponding to the behaviour of a lumped system. The fact that the internal resistance is an order of magnitude higher than the interface resistance informs us, that for high intensity heat pulses the temperature in some parts of the electrode could reach a value higher than $100 \mathrm{mK}$.

The heat load supplied to the electrode in transient measurements reflects approximately the future conditions of the electrodes in the AEgIS experiment. An estimated heat load of $10^{-5} \mathrm{~J}$ in one micro second corresponds to an enormous power of $10 \mathrm{~W}$. An assumption is made that the heat load will spread over a time scale in the order of 1 second, which seems reasonable and nec- 
essary. A response of the electrode is, therefore, measured for three types of step function heat loads lasting: $0.25 \mathrm{~s}, 2 \mathrm{~s}$ and quasi-steady-state of $100 \mathrm{~s}$. The power was adjusted in such a way that the corresponding integrated amount of energy deposited in each case equals to $10^{-5} \mathrm{~J}$.

The maximum temperature of the electrode is an interesting parameter for its application in the AEgIS experiment. The maximum measured temperature is higher at the top sector than at the side one, which is closer to the heat sink. The maximum temperature is also higher for higher base temperatures. At the lowest measured base mixing chamber temperature of $17 \mathrm{mK}$, the maximum temperature of the sectors never exceeds $100 \mathrm{mK}$ (figure 7.14 on the left). For the quasi steady state pulse the temperature rises to maximum $80 \mathrm{mK}$ at the highest base temperature of $70 \mathrm{mK}$. For the shortest heat pulse of $0.25 \mathrm{~s}$ the recorded temperature did not exceed $130 \mathrm{mK}$ at the highest measured base temperature of $70 \mathrm{mK}$. However, the heat in the AEgIS will be distributed more uniformly than with a single heater in the top sector, what should result in a lower maximum temperature.

For the measurement points exceeding $100 \mathrm{mK}$, it is worth to show how long that sensor actually has a temperature higher than $100 \mathrm{mK}$, which is presented in figure 7.14 on the right. The side sector of the electrode is warmer than $100 \mathrm{mK}$ for no longer than $5 \mathrm{~s}$ out of $100 \mathrm{~s}$ of the total cycle duration, even for the most intense pulses. The lower the base temperature, the shorter the time above $100 \mathrm{mK}$. The top sector, where the heat was deposited, is warmer than $100 \mathrm{mK}$ for no longer than $12 \mathrm{~s}$, and that time decreases significantly with a decrease of the base temperature.

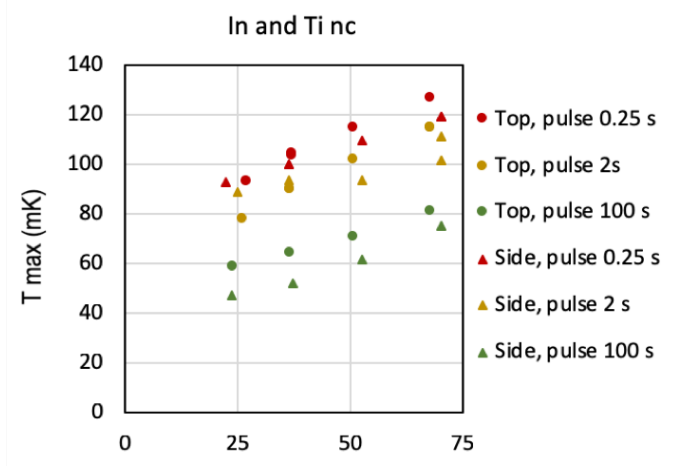

Equilibrium temperature (mK)

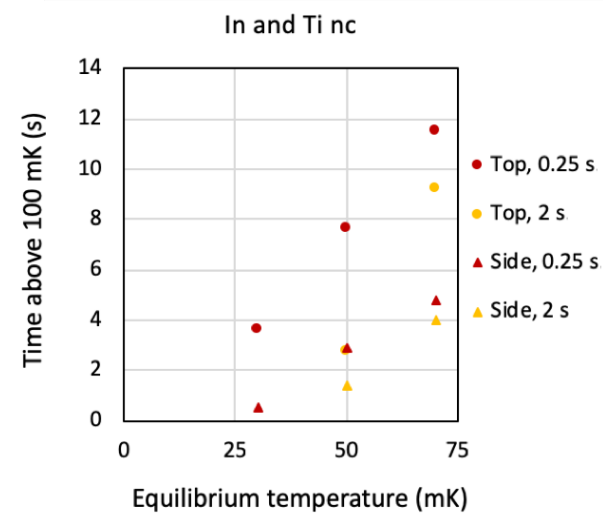

Figure 7.14: Left: the maximum temperature recorded on the electrode during transient measurements for the top and side sectors at various equilibrium temperatures and durations of the heat pulse, with indium and titanium in the superconducting state. Right: the time when the temperature of the sectors is higher than $100 \mathrm{mK}$ as a function of the equilibrium temperature for different heat pulse durations. For the heat pulse of $100 \mathrm{~s}$ the maximum temperature has never reached $100 \mathrm{mK}$. 
As demonstrated in a series of measurements, the electrodes could stay below $100 \mathrm{mK}$ if kept at a sufficiently low base temperature of $20 \mathrm{mK}-25 \mathrm{mK}$. For higher base temperatures, the maximum temperature of some sectors can exceed $100 \mathrm{mK}$ only for a few seconds after the arrival of the heat pulse. In the AEgIS experiment the heat will be deposited on the electrodes in a more uniform manner than only in one sector, what would make the temperature distribution more uniform and possibly result in an excursion to a maximum temperature lower than $100 \mathrm{mK}$ even at higher base temperatures.

\subsection{Outlook}

The important conclusion from chapter 6 is, that when indium is in the normal conducting state the clamping force of the sapphire - indium - copper sandwich can be removed without any significant degradation of the interface thermal properties. Thanks to this information and therefore the possibility of omitting the clamping structure of the electrodes, the design of the electrodes could be modified significantly.

The newly designed electrode included a series of improvements. The shape of the sapphire was modified adding material in the critical parts, to avoid the effect of a thermal bottle-neck in the smallest cross-section. The area of the bottom surface was increased, and the presence of several thin layers eliminated, which highly improved the thermal contact between the electrode and the thermalisation plate. The mounting strategy was also modified, providing a more uniform pressure distribution at the bottom surface of the electrode, and improving the quality of the indium bond.

The newly designed electrode can transfer a heat flow of $1.2 \mu \mathrm{W}$ while remaining at $100 \mathrm{mK}$ (measured at the side sector) when the thermalisation plate is at $50 \mathrm{mK}$. Even more heat can be transferred when the thermalisation plate is kept at a lower temperature. In transient conditions the electrode can extract the requested amount of energy of $10 \mu \mathrm{J}$, deposited in a time scale of approximately $1 \mathrm{~s}$, without increasing the temperature above $100 \mathrm{mK}$, if kept at a base temperature of approximately $25 \mathrm{mK}$. For higher base temperatures, the temperatures of some sectors can increase above $100 \mathrm{mK}$ but only for a few $(<12)$ seconds out of the $100 \mathrm{~s}$ cycle. During the rest of the cycle time the electrode remains below $100 \mathrm{mK}$.

The way in which the electrode was mounted during the cold weld formation at room temperature can easily be applied to the package of several electrodes mounted at the same time to the thermalisation plate in the AEgIS experiment. The presence of a cylinder pressing the electrodes in the middle will help obtain a proper alignment of the whole series of electrodes. The indium bonding is a sufficient fixation of the electrodes in the AEgIS experiment. However, one should keep in mind the particular properties of the cold weld. Its mechanical 
strength is the highest and reaches reasonably high values [5,6], when charged with a purely tensile load. Shear stress caused by bending, torsion, or a side force is very destructive. Therefore, any unnecessary manipulation involving the electrodes should be avoided after the removal of the clamping structure.

Small improvements, not having any significant influence on the heat transfer, can still be made, e.g. the shape of the four golden sectors could be slightly modified (e.g. the bottom sector could be a bit higher) to obtain a better access to all of the sectors from the sides of the electrodes package, in order to facilitate the attachment of the electrical connections. 


\section{Chapter 8}

\section{Conclusions}

The work presented in this thesis was motivated by the challenge encountered in the AEgIS project. The aim of the AEgIS project is the first direct measurement of the Earth's gravitational field on antimatter. To achieve this goal within $1 \%$ precision, antihydrogen must be cooled down below $100 \mathrm{mK}$ to minimize the thermal movement of the particles. The ultra-cold electrodes, where the antihydrogen formation takes place, were designed in accordance with a series of very restrictive requirements. Based on the previous experiments conducted in the CERN Central Cryogenic Laboratory, all factors influencing the thermal performance of the electrodes, which should be made by depositing four golden sectors on a sapphire base, have been profoundly analysed and several further measurement campaigns have been conducted.

The properties of the interface, responsible for the thermal anchoring of the electrodes to the mixing chamber of a dilution refrigerator, have been studied. The influence of the thin layers of gold, titanium and indium, with titanium and indium in the normal and superconducting states, have been evaluated. The interface thermal resistivity of various dielectric-metallic connections was modelled and measured in compliance with the definition of the Acoustic Mismatch Model.

The first sandwich setup measured in the CERN Cryolab Dilution Refrigerator consisted of sapphire, indium and copper. It represents the minimum amount of materials and interfaces necessary to guarantee the requested features of the electrodes. The main conclusion from the analysis of this setup is that once a proper indium bond between the sapphire and copper has been obtained, when indium is in the normal conducting state, the thermal properties do not change significantly depending on the presence of the compression force. Therefore, the clamping structure can be removed from the electrode leaving some precious space for the instrumentation. The fact that the clamping structure can be removed changes significantly also the way the electrodes are attached to the thermalisation plate. 
The measured thermal resistivity of the sapphire - indium - copper sandwich with indium in the normal conducting state takes a value from $17 \mathrm{~cm}^{2} \mathrm{~K}^{4} / \mathrm{W}$ at the lowest measured temperature of approximately $30 \mathrm{mK}$, to $44 \mathrm{~cm}^{2} \mathrm{~K}^{4} / \mathrm{W}$ at $375 \mathrm{mK}$ stamp temperature. The thermal resistivity of the same setup with indium in the superconducting state takes a value from approximately $100 \mathrm{~cm}^{2} \mathrm{~K}^{4} / \mathrm{W}$ at $50 \mathrm{mK}$ to $163 \mathrm{~cm}^{2} \mathrm{~K}^{4} / \mathrm{W}$ at $340 \mathrm{mK}$. The increase of the thermal resistivity of the sandwich is explained by a doubled number of dielectric - metallic interfaces. The validity of the parallel plates assumption at the sapphire disk has been shown (chapter 5). Including the parallel plates assumption, the measured value of the interface thermal resistivity is consistent with the prediction of the AMM at the lowest temperatures. The effect of alloying and ageing of the copper - indium interface, increasing the interface thermal resistivity, has been observed (chapter 6).

The second measured sandwich consisted of sapphire, titanium, gold, indium and copper. Despite having twice more interfaces than the first setup, the theoretical estimation of the thermal resistivity for the second setup gives a lower value, because of smaller differences between the acoustic impedances of neighbouring materials. The measured total thermal resistivity of the setup takes a value from $28 \mathrm{~cm}^{2} \mathrm{~K}^{4} / \mathrm{W}$ at $38 \mathrm{mK}$ to $77 \mathrm{~cm}^{2} \mathrm{~K}^{4} / \mathrm{W}$ at $407 \mathrm{mK}$. It is questionable whether the parallel plates assumption remains valid for a sapphire disk with layers of $\mathrm{TiO}$ at interfaces to titanium, since the measured thermal resistivity is higher than the theoretical prediction and higher than the value measured for the first setup. The thin layers, of a thickness lower than the wavelength of phonons at ultra-low temperatures, have been found to be the main reason for the increase of the thermal resistivity. The interlayer responsible for the adhesion of titanium to sapphire and the intermetallic compounds between all the metallic layers additionally contribute to the total thermal resistivity.

The significance of the thin layers is even more visible when indium and titanium are in the superconducting state and the electrons are not available for the heat transport. The measured thermal resistivity of the second sandwich setup with the layers of indium and titanium in the superconducting state takes a value from $187 \mathrm{~cm}^{2} \mathrm{~K}^{4} / \mathrm{W}$ at $63 \mathrm{mK}$ to $480 \mathrm{~cm}^{2} \mathrm{~K}^{4} / \mathrm{W}$ at $400 \mathrm{mK}$ stamp temperature, at which temperature titanium becomes normal conducting and the resistivity decreases.

The mathematical model of both setups was built. It contains all the thin layers and interfaces present in the real sandwich setups and allows an exact identification of the contributions from each thin layer and interface to the total thermal resistivity. The model shows that the effective thermal conductivity of a $45 \mathrm{~nm}$ layer of titanium has a magnitude similar to the Kapitza-like interface resistivity. This is important information for all kinds of thermal designs working in ultra-low temperature conditions. It also indicates that the electrodes forming the Penning trap in the AEgIS experiment should be designed avoid- 
ing the deposition of thin layers of titanium and gold on the bottom interface responsible for the thermal anchoring.

The sandwich setups have been measured also in the transient mode on a pulse-tube cryocooler and in the Cryolab DR. The thermal diffusivity was found to be frequency dependent [114]. That surprising result has been explained by a profound analysis and verification of the measurement method. The measurement result depends on the thermal link between the setup and the heat sink. The minimum temperature dependent threshold frequency $f_{\mathrm{c} 1}$, that enables correct transient measurements, has been introduced [72]. The measured thermal diffusivity increases with a decrease of temperature in the temperature range from approximately $6 \mathrm{~K}$ to $30 \mathrm{~K}$. The diffusivity of the sapphire - indium - copper sandwich is approximately one order of magnitude higher than the diffusivity of the setup with thin layers of titanium and gold. The diffusivity of the first setup takes the highest value of approximately $200 \mathrm{~cm}^{2} / \mathrm{s}$ at $6 \mathrm{~K}$, and the diffusivity of the second one rises to the maximal value of $100 \mathrm{~cm}^{2} / \mathrm{s}$ at $3 \mathrm{~K}$. Both curves seem to be merging towards the low temperature end at around $3 \mathrm{~K}$ (fig. 6.20), where the diffusivity starts decreasing. Only four reliable measurement points have been taken at ultra-low temperatures for the setup with thin layers of $\mathrm{Ti}$ and $\mathrm{Au}$. The measured values were $0.5 \mathrm{~cm}^{2} / \mathrm{s}$ at a base temperature of $45 \mathrm{mK}$ and $1.5 \mathrm{~cm}^{2} / \mathrm{s}$ at $93 \mathrm{mK}$ with Ti and $\mathrm{In}$ in the normal conducting state; and $1.2 \mathrm{~cm}^{2} / \mathrm{s}$ at $230 \mathrm{mK}$ and $1.4 \mathrm{~cm}^{2} / \mathrm{s}$ at $290 \mathrm{mK}$ for $\mathrm{Ti}$ and $\mathrm{In}$ in the superconducting state. The values measured at ultra-low temperatures are much lower than in the low temperature range, but they fit quite well the overall theoretical prediction obtained from the model (fig. 6.29).

Based on the findings from the ultra-low temperature measurements and the modelling, two new ultra-cold electrodes for the AEgIS experiment have been designed and manufactured. The new design included also results of a mechanical stress analysis. Thanks to the fact that the clamping structure can be removed after the indium bond is obtained, without a significant degradation of the electrode performance during its operation, the shape of the new electrodes could be modified to increase the area of the smallest cross-section in the heat pathway. The increased amount of material in the smallest cross-section minimizes mechanical stresses in that place to avoid an additional limitation of the heat flow. The four sectors of the electrodes have been deposited with a magnetron sputtering technique on the sapphire base with a very high precision. The layer of indium has been vapour deposited on the optically polished bottom surfaces of the electrodes, paying attention not to merge the deposition with the lowest sectors of the electrodes. One of the electrodes has been attached to the thermalisation plate with an indium bond. Thanks to the new design of the clamping structure, the pressure distribution is very uniform, making the quality of the indium bond better at the whole surface.

The thermal performance of the electrode has been measured in the Cryolab DR. The steady state measurements show that the electrode can transfer 
$1.2 \mu \mathrm{W}$, when the temperature measured on the side of the electrode does not exceed $100 \mathrm{mK}$, and the thermalisation plate is kept at $50 \mathrm{mK}$. This is more than 5 times the amount of the heat that could be transferred by the old design of the electrode at the same temperatures. The transient behaviour of the electrode has also been measured as a response to the heat pulse of $10 \mu \mathrm{J}$ deposited in different time intervals. For the heat pulse duration of $2 \mathrm{~s}$ or even $0.25 \mathrm{~s}$, the temperature of the electrode never rises above $100 \mathrm{mK}$, if it is kept at an equilibrium temperature of about $25 \mathrm{mK}$, i.e. the mixing chamber at $17 \mathrm{mK}$.

The new design of the electrode fulfils all of the severe demands that have been formulated in chapter 1. The electrodes can be applied in the AEgIS project with some minor changes, e.g. to facilitate the attachment of the wiring to the four sectors, that create the quadrupole electric field of the Penning trap. Thanks to the new way of mounting the electrodes on the thermalisation plate, the whole package of 10 electrodes of different thicknesses can be easily aligned, attached and tested. 
Appendix 

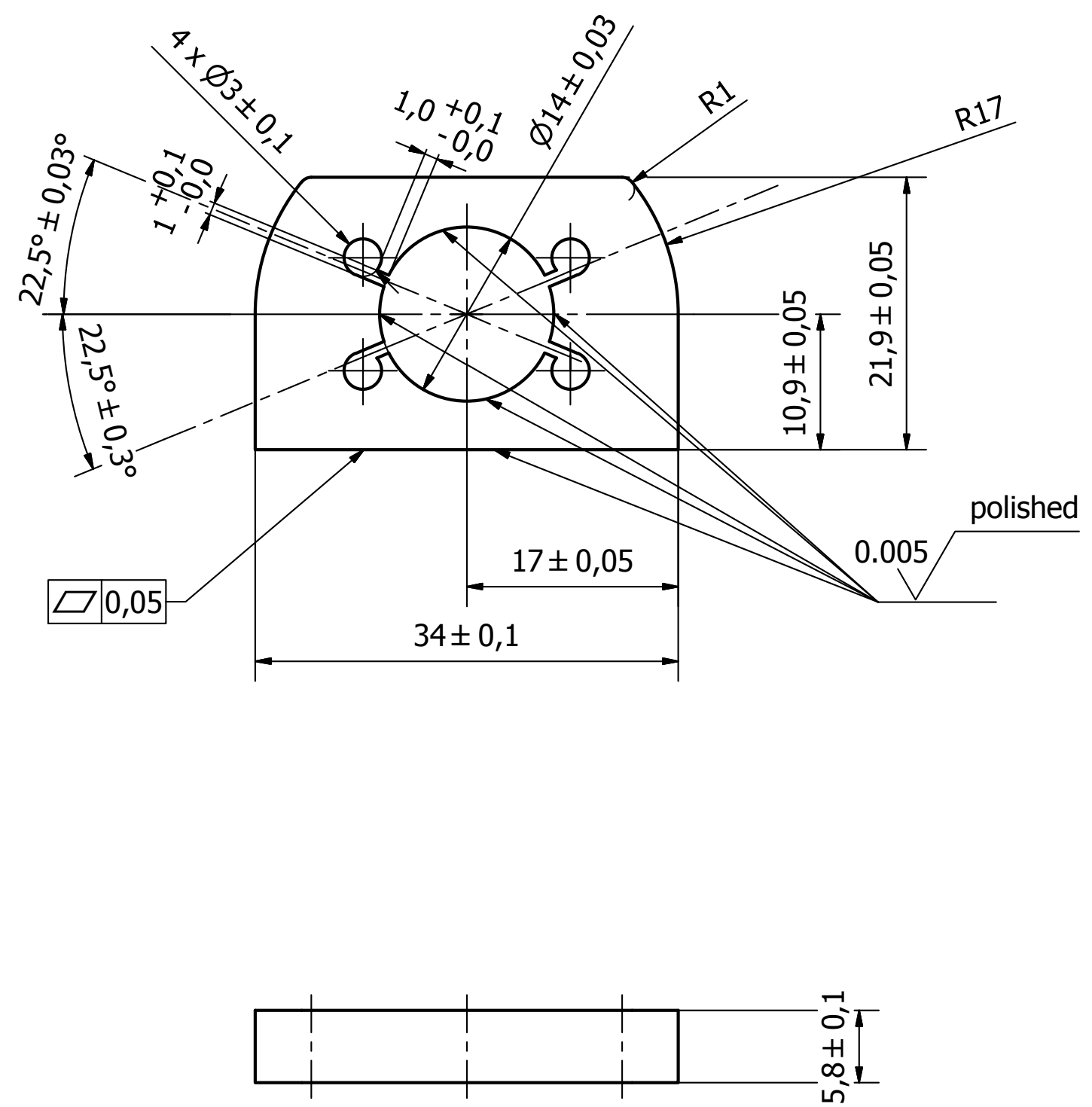

\begin{tabular}{|l|l|l|l|l|l|}
\hline $\begin{array}{l}\text { Designed by } \\
\text { jliberad }\end{array}$ & Checked by & Approved by & \multicolumn{1}{|l|}{$\begin{array}{l}\text { Format } \\
\text { A4 }\end{array}$} & $\begin{array}{l}\text { Date } \\
13 / 09 / 2017\end{array}$ & $2: 1$ \\
\hline $\begin{array}{l}\text { Version 1.1: } \\
\text { height 21.9 mm }\end{array}$ & Sapphire & Edition & $\begin{array}{r}\text { Sheet } \\
1 / 1\end{array}$ \\
\cline { 2 - 5 } & & electrode_W34_v1 & & \\
\hline
\end{tabular}




\section{Bibliography}

[1] Drobychev G Y et al. 2007 Proposal for the AEGIS experiment at the CERN antiproton decelerator (Antimatter Experiment: Gravity, Interferometry, Spectroscopy) Tech. Rep. SPSC-P-334. CERN-SPSC-2007-017 CERN Geneva URL https://cds.cern.ch/record/1037532

[2] Brusa R S et al. 2017 Journal of Physics: Conference Series 791012014 URL http://stacks . iop.org/1742-6596/791/i=1/a=012014

[3] Kellerbauer A et al. 2008 Nuclear Instruments and Methods in Physics Research Section B: Beam Interactions with Materials and Atoms 266351 - 356 ISSN 0168-583X low Energy Positron and Positronium Physics URL http://www.sciencedirect.com/science/article/ pii/S0168583X07017740

[4] Eisel T 2011 Cooling of electrically insulated high voltage electrodes down to $30 \mathrm{mK}$ Ph.D. thesis Dresden, Tech. U. URL http://inspirehep. net/record/1231380/files/CERN-THESIS-2011-139.pdf

[5] Moore A C and Tabor D 1952 British Journal of Applied Physics 3299 URL http://stacks . iop.org/0508-3443/3/i=9/a=308

[6] Indium Corporation ${ }^{\circledR}$ Indium cold welding URL http://www.indium. com/blog/cold-welding-indium-metal.php

[7] Derking J, Liberadzka J, Koettig T and Bremer J 2015 Physics Procedia 67576 - 581 ISSN 1875-3892 proceedings of the ICEC25-ICMC 2014

[8] Alterovitz S, Deutscher G and Gershenson M 1975 Journal of Applied Physics 46 3637-3643 (Preprint https://doi.org/10.1063/1.322091) URL https://doi.org/10.1063/1.322091

[9] Boerstoel B, Zwart J and Hansen J 1971 Physica 54442 - 458 ISSN 0031-8914 URL http://www.sciencedirect.com/science/article/ pii/0031891471901893

[10] Chandrasekhar B S and Rayne J A 1961 Phys. Rev. 124(4) 1011-1014 URL https://link.aps .org/doi/10.1103/PhysRev.124.1011 
[11] Davey G and Mendelssohn K 1963 Physics Letters 7183 - 184 ISSN 0031-9163 URL http://wwW.sciencedirect.com/science/article/ pii/0031916363903755

[12] Ditmars D, Ishihara S, Chang S, Bernstein G and West E 1982 Journal of Research of the National Bureau of Standards $\mathbf{8 7} 159$

[13] Fugate R Q and Swenson C A 1969 Journal of Applied Physics 40 30343036 URL https://doi.org/10.1063/1.1658118

[14] Flik M I and Tien C L 1990 Journal of Heat Transfer 112 872-881 URL http://dx.doi.org/10.1115/1.2910494

[15] de Haas W and Biermasz T 1938 Physica 5 619 - 624 ISSN 0031-8914 URL http://wWw.sciencedirect.com/science/article/ pii/S0031891438800094

[16] Lounasmaa O V 1974 Experimental principles and methods below $1 \mathrm{~K}$ (London: Academic Press) ISBN 01245595069780124559509

[17] Mamiya T, Yano H, Uchiyama T, Inoue S and Miura Y 1988 Review of Scientific Instruments 59 1428-1430 URL https://doi.org/10.1063/ 1.1139684

[18] Neeper D A and Dillinger J R 1964 Phys. Rev. 135(4A) A1028-A1033 URL https://link.aps.org/doi/10.1103/PhysRev.135.A1028

[19] Rayne J A 1956 Australian Journal of Physics 9189

[20] Schmidt G 1974 Physics Letters A $50241 \quad$ - 242 ISSN 03759601 URL http://www.sciencedirect.com/science/article/pii/ 0375960174907336

[21] Schmidt C and Umlauf E 1976 Journal of Low Temperature Physics 22 597-611 ISSN 1573-7357 URL https://doi.org/10.1007/BF00659062

[22] Schmidt C 1977 Phys. Rev. B 15(9) 4187-4192 URL https://link. aps.org/doi/10.1103/PhysRevB.15.4187

[23] Swenson C A 1999 Review of Scientific Instruments 70 2728-2731 URL https://doi.org/10.1063/1.1149836

[24] Viswanathan R 1975 Journal of Applied Physics 46 4086-4087 URL https://doi.org/10.1063/1.322119

[25] Wolfmeyer M, Fox G and Dillinger J 1970 Physics Letters A 31401 402 ISSN 0375-9601 URL http://www.sciencedirect.com/science/ article/pii/0375960170910091 
[26] Wolfmeyer M and Dillinger J 1971 Physics Letters A 34247 - 248 ISSN 0375-9601 URL http://www.sciencedirect.com/science/article/ pii/037596017190853X

[27] Bintley D, Woodcraft A L and Gannaway F C 2007 Cryogenics 47333 - 342 ISSN 0011-2275 URL http://www.sciencedirect.com/science/ article/pii/S0011227507000458

[28] Mittag K 1973 Cryogenics 13 94-99

[29] Debye P 1912 Annalen der Physik 344 789-839

[30] Kittel C 2004 Introduction to Solid State Physics (Wiley) ISBN 9780471415268 URL https://books.google.fr/books?id= kym4QgAACAAJ

[31] Enss C and Hunklinger S 2005 Low-Temperature Physics (Springer Berlin Heidelberg) URL https://books.google.fr/books?id=ufM7sPMTGdAC

[32] von Gutfeld R J and Nethercot A H 1966 Phys. Rev. Lett. 17(16) 868-871 URL https : //link . aps .org/doi/10.1103/PhysRevLett.17.868

[33] White G K 1953 Proceedings of the Physical Society. Section A 66559 URL http: //stacks . iop.org/0370-1298/66/i=6/a=307

[34] Mendelssohn K and Rosenberg H M 1952 Proceedings of the Physical Society. Section A 65385 URL http://stacks.iop.org/0370-1298/ $65 / i=6 / a=301$

[35] Mendelssohn K and Rosenberg H M 1952 Proceedings of the Physical Society. Section A 65388 URL http://stacks.iop.org/0370-1298/ $65 / i=6 / a=302$

[36] Kemp W R G, Klemens P G and White G K 1956 Australian Journal of Physics 9180

[37] Nath P and Chopra K 1974 Thin Solid Films 2053 - 62 ISSN 0040-6090 URL http://wWw.sciencedirect.com/science/article/ pii/0040609074900339

[38] Pobell F 2007 Matter and Methods at Low Temperatures (Springer Berlin Heidelberg) ISBN 9783540463603

[39] Onnes, H Kamerlingh 1991 Further experiments with Liquid Helium. G. On the Electrical Resistance of Pure Metals, etc. VI. On the Sudden Change in the Rate at which the Resistance of Mercury Disappears. (Dordrecht: Springer Netherlands) pp 267-272 ISBN 978-94-009-2079-8 
[40] Cyrot M 1973 Reports on Progress in Physics 36103 URL http:// stacks.iop.org/0034-4885/36/i=2/a=001

[41] Bardeen J, Cooper L N and Schrieffer J R 1957 Phys. Rev. 106(1) 162164 URL https://link .aps .org/doi/10.1103/PhysRev.106.162

[42] Jones R E and Toxen A M 1960 Phys. Rev. 120(4) 1167-1170 URL https://link.aps.org/doi/10.1103/PhysRev.120.1167

[43] Yousept I 2017 SIAM Journal on Control and Optimization 55 23052332

[44] URL https://commons.wikimedia.org/wiki/File:EXPULSION.png

[45] Kapitza P L 1941 Phys. Rev. 60(4) 354-355

[46] Khalatnikov I 1952 Zhurnal Eksperimentalnoi I Teoreticheskoi Fiziki 22 687-704

[47] Little W 1959 Canadian Journal of Physics

[48] Rumble J R, Lide D R and Bruno T J 2018 CRC handbook of chemistry and physics : a ready-reference book of chemical and physical data. (Boca Raton, FL : Taylor and Francis, an imprint of CRC Press) ISBN 97811385616321138561630

[49] Neighbours J R and Alers G A 1958 Phys. Rev. 111(3) 707-712 URL https://link.aps.org/doi/10.1103/PhysRev.111.707

[50] GE Infrastructure 2004 Sound Speeds and Pipe Size Data URL http://www.instrumart.com/assets/ ge-sound-speeds-and-pipe-size-data.pdf

[51] Papk B S and Narahara Y 1971 Journal of the Physical Society of Japan 30

[52] Swartz E T 1987 Solid-Solid Thermal Boundary Resistance. Ph.D. thesis Cornell University

[53] Swartz E and Pohl R 1989 Reviews of Modern Physics 61 605-668 ISSN 0034-6861

[54] Hopkins P E and Norris P M 2007 Nanoscale and Microscale Thermophysical Engineering 11 247-257 URL https://doi.org/10.1080/ 15567260701715297

[55] Hopkins P E 2009 Journal of Applied Physics 106013528 URL https: //doi.org/10.1063/1.3169515 
[56] Zhan T, Fang L and Xu Y 2017 Scientific Reports 77109 URL https: //doi.org/10.1038/s41598-017-07150-7

[57] Hsu T 2008 MEMS and Microsystems: Design, Manufacture, and Nanoscale Engineering (Wiley) ISBN 9780470083017

[58] Flik M I, Choi B I and Goodson K E 1992 Journal of Heat Transfer 114 666-674 URL http://dx.doi.org/10.1115/1.2911332

[59] Flik M I and Tien C L 1990 Journal of Heat Transfer 112 872-881 URL http://dx.doi.org/10.1115/1.2910494

[60] Turney J E, McGaughey A J H and Amon C H 2010 Journal of Applied Physics 107024317

[61] Heino P 2007 The European Physical Journal B 60 171-179 ISSN 14346036

[62] Langer G, Hartmann J and Reichling M 1997 Review of Scientific Instruments 68 1510-1513 URL https://doi.org/10.1063/1.1147638

[63] Majumdar A 1993 Journal of Heat Transfer 115 7-16 URL http://dx. doi.org/10.1115/1.2910673

[64] Salemink H, Van Kempen H and Wider P 1978 Journal de Physique Colloques 39 C6-326-C6-327 URL https://hal .archives-ouvertes. fr/jpa-00217552

[65] Wang X and Huang B 2014 Scientific Reports 4

[66] Gusev A V, Gibin A M, Morozkin O N, Gavva V A and Mitin A V 2002 Inorganic Materials 38 1100-1102 ISSN 1608-3172 URL https: //doi.org/10.1023/A:1020902213235

[67] Glassbrenner C J and Slack G A 1964 Phys. Rev. 134(4A) A1058-A1069 URL https://link.aps .org/doi/10.1103/PhysRev.134.A1058

[68] Lunt, A 2018 Scanning Transmission Electron Microscopy and Energy Dispersive Spectroscopy of Thin Films on Sapphire Disks for Heat Transfer Measurements Tech. Rep. EDMS 2019351 v.1 CERN TS-MME-MM Geneva

[69] Oxford Instruments URL https://nanoscience.oxinst.com

[70] Godfrin, Henri and Gianèse, Christian 2014 Reflets phys. 19-21 URL https://doi.org/10.1051/refdp/201439019 
[71] Ventura G and Risegari L 2010 The Art of Cryogenics: Low-Temperature Experimental Techniques (Elsevier Science) ISBN 9780080554365 URL https://books.google.fr/books?id=4kvzBRUuGDkC

[72] Golm J 2018 Study of Thermal Diffusivity of Dielectric-Metal Sandwich Structures at Low Temperatures URL http://cdsweb.cern.ch/ record/2634825/files/CERN-THESIS-2018-116.pdf

[73] Wikus P and Niinikoski T O 2007 Dilution Refrigeration of Multi-Ton Cold Masses Ph.D. thesis Wien TU presented on 13 Jun 2007 URL http: //cds.cern.ch/record/1036217

[74] Koettig T, Maciocha W, Bermudez S, Rysti J, Tavares S, Cacherat F and Bremer J 2017 IOP Conference Series: Materials Science and Engineering 171012103 URL http://stacks.iop.org/1757-899X/171/i= $1 / \mathrm{a}=012103$

[75] Dubuis G, He X and Božović I 2014 Review of Scientific Instruments 85 103902 URL https://doi.org/10.1063/1.4896049

[76] Picowatt TS-530A Temperature Controller Instruction Manual URL http://www.picowatt.fi/support/530a_man.pdf

[77] Peterson R E and Anderson A C 1973 Journal of Low Temperature Physics 11 639-665 ISSN 1573-7357

[78] Coops G, De Waele A and Gijsman H 1979 Cryogenics 19 659-665

[79] LakeShore URL https://www. lakeshore.com

[80] LakeShore RuO sensor U02611 Calibration Curve EDMS 1394714 v.1 URL https : //edms . cern . ch/document/1394714/1

[81] LakeShore RuO sensor U01826 Calibration Curve EDMS 1394670 v.1 URL https://edms . cern.ch/document/1394670/1

[82] van der Veur, Wiemer 2009 Calibration of RuO2 temperature sensors in the $25 \mathrm{mK}-1 \mathrm{~K}$ range

[83] A Bosch W, Chinchure A, Flokstra J, de Groot M, Jochemsen R, Mathu F, Peruzzi A and Veldhuis D 2018 Status report on the development of a superconducting reference device for precision thermometry below $1 \mathrm{k}$ Tech. rep.

[84] Hightech Development Leiden URL https://hdleiden.home.xs4all. nl/srd1000/ 
[85] Test data SRD1000-6 S301 EDMS 2029415 v.1 URL https://edms. cern.ch/document/2029415/1

[86] Apiezon N Grease URL https://www.apiezon.com/products/ vacuum-greases/n-grease

[87] Goodrich R, Hall D, Palm E and Murphy T 1998 Cryogenics 38221 - 225 ISSN 0011-2275 URL http://www.sciencedirect.com/science/ article/pii/S0011227597001008

[88] Żak D, Dziedzic A, Kolek A, Stadler A W, Mleczko K, Szałański P and Zawiślak Z 2006 Measurement Science and Technology 1722 URL http: //stacks.iop.org/0957-0233/17/i=1/a=005

[89] Jorba P, Piatek J and Ronnow H 2013 Calibration of RuO2 commercially available resistors for temperature sensing at dilution fridge temperatures Tech. rep. EPFL

[90] LakeShore Appendix D URL https://www. lakeshore.com/Documents/ LSTC_appendixD_l.pdf

[91] Picowatt URL http://www.picowatt.fi

[92] Erlebach, $\mathrm{R}$ Thermohaus University of Jena URL http: //www.personal.uni-jena.de/ p1erra/physik/protokolle/seite\% 20129-\%20versuch\%20210.pdf

[93] Jensen J E, Stewart R G, Tuttle W A, Brechna H, Laboratory B N and Commission U S A E 1980 Brookhaven National Laboratory selected cryogenic data notebook ([New York]: Brookhaven National Laboratory)

[94] Borges de Sousa, P 2017 Thermal conductivity of impregnated Nb3Sn coil samples Tech. Rep. EDMS 1871957 v.1 CERN TE-CRG-CI Geneva

[95] Frossati G 1992 Journal of Low Temperature Physics 87 595-633 ISSN 1573-7357 URL https ://doi .org/10.1007/BF00114918

[96] Frey H and Haefer R 1981 Tieftemperaturtechnologie (VDI-Verlag) ISBN 9783184005030 URL https://books.google.ch/books?id= UGjYAAAACAAJ

[97] Chen G 2005 Nanoscale Energy Transport and Conversion: A Parallel Treatment of Electrons, Molecules, Phonons, and Photons MITPappalardo Series in Mechanical Engineering (Oxford University Press) ISBN 9780199774685 URL https://books.google.ch/books? id=M3n31UJpYDYC 
[98] Ho P C and Hallock R B 2000 Journal of Low Temperature Physics 121 797-802 ISSN 1573-7357 URL https://doi.org/10.1023/A: 1017581409634

[99] Winder D and Smith C S 1958 Journal of Physics and Chemistry of Solids 4128 - 134 ISSN 0022-3697 URL http://wWw.sciencedirect. com/science/article/pii/0022369758902026

[100] Balandin A and Wang K L 1998 Phys. Rev. B 58(3) 1544-1549 URL https://link.aps.org/doi/10.1103/PhysRevB.58.1544

[101] Fuchs K 1938 Mathematical Proceedings of the Cambridge Philosophical Society 34 100-108

[102] Toxen A M 1961 Phys. Rev. 123(2) 442-446 URL https://link.aps. org/doi/10.1103/PhysRev.123.442

[103] Matsumoto D S, Reynolds C L and Anderson A C 1977 Phys. Rev. B 16(8) 3303-3307 URL https://link.aps.org/doi/10.1103/ PhysRevB.16.3303

[104] Reynolds C L and Anderson A C 1975 Journal of Low Temperature Physics 21 641-643 ISSN 1573-7357 URL https://doi.org/10.1007/ BF01141615

[105] Selverian J H, Ohuchi F S, Bortz M and Notis M R 1991 Journal of Materials Science 26 6300-6308 ISSN 1573-4803 URL https://doi. org/10.1007/BF02387808

[106] Steele M C and Hein R A 1953 Phys. Rev. 92(2) 243-247 URL https: //link.aps.org/doi/10.1103/PhysRev.92.243

[107] Martinez W E, Gregori G and Mates T 2010 Thin Solid Films 5182585 2591 ISSN 0040-6090 URL http://www.sciencedirect.com/science/ article/pii/S0040609009013376

[108] Ekin J W 2015 Experimental techniques for low-temperature measurements: cryostat design, material properties, and superconductor criticalcurrent testing ISBN 97801985705470198570546

[109] Berman R, Foster E L and Ziman J M 1955 Proceedings of the Royal Society of London A: Mathematical, Physical and Engineering Sciences 231 130-144 ISSN 0080-4630 URL http://rspa.royalsocietypublishing. org/content/231/1184/130

[110] Cotts E J and Anderson A C 1981 Journal of Low Temperature Physics 43 437-443 ISSN 1573-7357 URL https://doi.org/10.1007/ BF00115606 
[111] Sellers G J and Anderson A C 1974 Review of Scientific Instruments 45 1256-1259 URL https://doi.org/10.1063/1.1686472

[112] Gmelin E, Asen-Palmer M, Reuther M and Villar R 1999 Journal of Physics D: Applied Physics 32 R19 URL http://stacks.iop.org/ $0022-3727 / 32 / i=6 / a=004$

[113] Voldman S 2005 ESD: Circuits and Devices (Wiley) ISBN 9780470847541 URL https://books.google.fr/books?id=25EyuAAACAAJ

[114] van der Post C C W 2016 Heat Transfer at Ultra-Low Temperature 


\section{Summary}

The AEgIS project is one of the experiments based in the Antimatter Factory of the European Organisation for Nuclear Research (CERN). Its goal is the first direct measurement of the Earth's gravitational acceleration on antimatter within $1 \%$ precision. In the framework of this project, a thermalisation strategy for a set of ultra-cold electrodes forming a Penning trap for antimatter has been investigated. The electrodes need to fulfill a series of requirements. Some of them must be divided into 4 sectors and the electrical insulation between the sectors and the neighbouring electrodes must withstand up to $1 \mathrm{kV}$ potential difference. Each electrode will be made of a single sapphire crystal and the four sectors will be sputtered with gold to provide a sufficient electrical insulation and at the same time proper thermal anchoring. Two sandwich setups reflecting two possible ways of anchoring the electrodes on a mixing chamber of a dilution refrigerator have been analysed. The first sandwich consisted of copper, indium and sapphire, and the second of copper, indium, gold, titanium and sapphire. Sapphire was always in the form of a flat polished disk of $1 \mathrm{~mm}$ thickness, and the metallic thin layers had following thicknesses: Ti $45 \mathrm{~nm}$, Au $520 \mathrm{~nm}$ and In $125 \mu \mathrm{m}$.

The thermal resistivity of both setups with and without an external magnetic field was measured. The total thermal resistivity of the first setup with indium in the normal conducting state takes a value from $17 \mathrm{~cm}^{2} \mathrm{~K}^{4} / \mathrm{W}$ to $44 \mathrm{~cm}^{2} \mathrm{~K}^{4} / \mathrm{W}$ in the temperature range from $30 \mathrm{mK}$ to $375 \mathrm{mK}$. With indium in the superconducting state, the total thermal resistivity is much higher and takes a value from $100 \mathrm{~cm}^{2} \mathrm{~K}^{4} / \mathrm{W}$ at $50 \mathrm{mK}$ to $163 \mathrm{~cm}^{2} \mathrm{~K}^{4} / \mathrm{W}$ at $340 \mathrm{mK}$. The investigation of the first sandwich has confirmed the validity of the parallel plates assumption at the sapphire disk, and demonstrated the importance of the oxide layers on the interface thermal resistance. The important conclusion has been drawn that with indium in the normal conducting state the compression force can be removed without changing the total thermal resistivity of the sandwich. It allowed a significant modification of the clamping structure and the shape of the electrode.

The total thermal resistivity of the second setup with thin layers of gold and titanium takes a value from $28 \mathrm{~cm}^{2} \mathrm{~K}^{4} / \mathrm{W}$ at $38 \mathrm{mK}$ to $77 \mathrm{~cm}^{2} \mathrm{~K}^{4} / \mathrm{W}$ at $407 \mathrm{mK}$ with titanium and indium in the normal conducting state. This 
result shows that sputtering of titanium and the mechanism of its adhesion to sapphire can have a significant influence on the interface thermal resistivity. The same setup with superconducting layers of titanium and indium has the highest thermal resistivity of all the measured configurations, which takes a value from $187 \mathrm{~cm}^{2} \mathrm{~K}^{4} / \mathrm{W}$ at $63 \mathrm{mK}$ to $480 \mathrm{~cm}^{2} \mathrm{~K}^{4} / \mathrm{W}$ at $400 \mathrm{mK}$. It shows a huge influence of the thin layers on the total thermal resistivity of the sandwich.

The low temperature and ultra-low temperature thermal diffusivity of the sandwich has also been measured. The results are consistent with respect to the built model over the whole temperature range, opening a possibility of a correct evaluation of the ultra-low temperature behaviour of the setup by measuring its low temperature properties. Such a conclusion is very important not only for the application in the AEgIS project, but also in any other system where the difficult to evaluate ultra-low temperature thermal performance plays a main role.

The new electrode has been designed taking into account findings from the ultra-low temperature measurements and conclusions drawn from the stress analysis. The electrode has been manufactured according to rigorous procedure to make sure it fulfils all of the demanding constraints. A new way of clamping the electrode has been designed. It provides a more uniform pressure distribution on the bottom surface of the electrode and increases the chances for a formation of a robust indium bond. The thermal performance of the electrode has been tested in steady state and transient conditions and it proved to be over five times higher than the performance of the old design. The newly designed electrode fulfils all the requirements and has a sufficient thermal performance for the application in the AEgIS experiment. 


\section{Samenvatting}

Het AEgIS project is één van de experimenten in de Antimatter Factory van de Europese Organisatie voor Nucleair Onderzoek (CERN). Het doel is het maken van de eerste directe meting van de zwaartekrachtversnelling van de Aarde op antimaterie binnen $1 \%$ nauwkeurigheid. In de context van dit project is een thermalisatie-strategie onderzocht voor een set ultrakoude elektroden die een Penning trap voor antimaterie vormen. De elekroden dienen aan een aantal eisen te voldoen. Sommigen moeten in vier sectoren worden verdeeld en de elektrische insulatie tussen de sectoren en de aangrenzende elektroden moeten een potentiaalverschil tot aan $1 \mathrm{kV}$ kunnen weerstaan. Elke elektrode zal gemaakt worden van een enkel saffierkristal en de vier sectoren worden gesputterd met goud om tegelijkertijd een toereikende elektrische insulatie en een fatsoenlijk thermisch anker te leveren. Er zijn twee sandwich-opstellingen onderzocht die twee mogelijke manieren reflecteren om de elektroden aan de mixkamer van een dilution refrigerator te verankeren. De eerste sandwich bestaat uit koper, indium en saffier, de tweede uit koper, indium, goud, titanium en saffier. Het saffier had altijd de vorm van een platte, gepolijste schijf van $1 \mathrm{~mm}$ dik. De dikte van de dunne metalen lagen was als volgt: Ti $45 \mathrm{~nm}$, Au $520 \mathrm{~nm}$, In $125 \mu \mathrm{m}$.

De warmteweerstand van beide opstellingen is zowel met als zonder een extern magnetisch veld gemeten. De totale warmteweerstand van de eerste opstelling met indium in de normaal geleidende staat loopt van $17 \mathrm{~cm}^{2} \mathrm{~K}^{4} / \mathrm{W}$ tot $44 \mathrm{~cm}^{2} \mathrm{~K}^{4} / \mathrm{W}$ binnen het temperatuurbereik $30 \mathrm{mK}$ tot $375 \mathrm{mK}$. Met indium in de supergeleidende staat is deze veel hoger, met een waarde van $100 \mathrm{~cm}^{2} \mathrm{~K}^{4} / \mathrm{W}$ bij $50 \mathrm{mK}$ tot $163 \mathrm{~cm}^{2} \mathrm{~K}^{4} / \mathrm{W}$ bij $340 \mathrm{mK}$. De analyse van de eerste sandwich bevestigde de geldigheid van de parallelle platen aanname bij de saffieren schijf en demonstreerde het belang van de oxidelagen op de warmteweerstand tussen de raakvlakken. De belangrijke conclusie is getrokken dat met indium in de normaal geleidende staat de drukkracht verwijderd kan worden zonder dat de totale warmteweerstand van de sandwich verandert. Dit maakte een aanzienlijke aanpassing van de klemstructuur en elektrodevorm mogelijk.

De totale warmteweerstand van de tweede opstelling, met dunne laagjes goud en titanium, neemt een waarde aan van $28 \mathrm{~cm}^{2} \mathrm{~K}^{4} / \mathrm{W}$ bij $38 \mathrm{mK}$ tot $77 \mathrm{~cm}^{2} \mathrm{~K}^{4} / \mathrm{W}$ bij $407 \mathrm{mK}$, met het titanium en indium in de normaal geleidende 
staat. Dit resultaat toont aan dat het sputteren van titanium en diens manier van hechten aan saffier een aanzienlijke invloed kan hebben op de thermische grensweerstand. Dezelfde opstelling met supergeleidende lagen titanium en indium heeft de hoogste warmteweerstand van alle gemeten configuraties, met een waarde van $187 \mathrm{~cm}^{2} \mathrm{~K}^{4} / \mathrm{W}$ bij $63 \mathrm{mK}$ tot $480 \mathrm{~cm}^{2} \mathrm{~K}^{4} / \mathrm{W}$ bij $400 \mathrm{mK}$. Dit laat een enorme invloed zien van de dunne lagen op de totale warmteweerstand van de sandwich.

Ook de thermische diffusiviteit van de sandwich bij lage en ultralage temperaturen is gemeten. De resultaten zijn consistent met betrekking tot het opgestelde model over het gehele temperatuursbereik, wat de mogelijkheid biedt tot een correcte evaluatie van het ultralage-temperatuursgedrag van de opstelling door het meten van diens lage-temperatuurseigenschappen. Een dergelijke conclusie is niet alleen zeer belangrijk voor de toepassing in het AegIS project, maar ook in elk ander systeem waar de moeilijk te evalueren thermische prestatie bij ultralage temperatuur een hoofdrol speelt.

De nieuwe elektrode is ontworpen met inachtname van bevindingen van de ultralage-temperatuursmetingen en conclusies getrokken uit de stressanalyse, en is gefabriceerd volgens een rigoreuze procedure om te zorgen dat het aan alle strenge eisen voldoet. Er is een nieuwe klemmethode ontworpen die een meer uniforme drukverdeling op de onderkant van de elektrode verschaft en de kans op een robuuste indiumverbinding verhoogt. De thermische prestatie van de elektrode is getest onder steady state en transiënte omstandigheden en bleek meer dan vijf keer hoger dan van het oude ontwerp. Hij voldoet aan alle eisen en heeft een voldoende thermische prestatie voor toepassing in het AEgIS experiment. 


\section{Acknowledgements}

Since I started my PhD, I have been living and working in a highly international environment, involving several countries and languages. Therefore, I will let myself write the acknowledgements in three languages, depending to whom they are directed.

Above all, I would like to thank my professor, Marcel ter Brake, and my daily supervisor, dr. Torsten Köttig, for their invaluable guidance that lead my PhD along a straight path from the beginning till the very end. Despite being not always easy, the way was always very well indicated. I especially thank Torsten for walking with me tirelessly through everyday numerous challenges and our fruitful discussions, and Marcel for his kind wisdom and for bringing my selfesteem to a decent level when it was really low. You both managed to be a supervisor and a friend at the same time, which is incredibly difficult and very impressive.

I would like to thank dr. Johan Bremer, CERN TE-CRG-CI section leader, for his trust in me, great support and for all the chances I have been given, including the opportunity of doing an interesting $\mathrm{PhD}$ in the friendly and educative atmosphere of the Cryolab.

Je voudrais remercier également toute l'équipe technique du Cryolab: Laetitia Dufay-Chanat, Agostino Vacca, Sebastien Prunet, Jean-Luc Carpano et Florian Girardot, pour leur amitié, aide et soutien constant. Vous avez souvent sauvé ma peau, grâce à votre sagesse et votre travail toujours rapide et précis. Merci !

I would like to thank also Miranda van Stenis and Thomas Schneider from CERN EP-DT-EF, and Wil Vollenberg from CERN TE-VSC-SCC for their friendly and helpful attitude, professional expertise and good advices. Without your successful depositions of indium, gold and titanium on the sapphire disks and the electrodes my task would never be accomplished!

I couldn't express sufficiently my gratitude to Jessica Golm and Casimir van der Post for the enormous work they have done working with me as a part of their master and bachelor theses, and for their excellent company during long evenings with the DR.

Not being familiar with the university world, I would have been completely 
lost without a guidance and help from Ans Veenstra-Lokin and Tim Mulder, so thank you very much for it.

Great thanks to my Cryolab friends, especially Patricia Borges de Sousa, Hernan Furci and Tiemo Winkler for our serious discussions about life and science, and Remy Kriboo for a smooth translation of my writing from English to Dutch. Patricia, thank you very much for your attention and your time spent reading this thesis and correcting my English!

Cristina Cristescu, Irina Savu, Valentina Venturi, Anne-Laure Lamure, Consuelo and Jens Spanggaard, Torsten Layda - my dear friends who have always been there ready to listen. You cured my pessimistic mood with your kind words, open hearts, and a profound understanding of problems more significant than a doctorate. I would never manage to express how much your presence and our conversations meant for me.

Dziękuję Rodzicom, Elżbiecie i Ireneuszowi Liberadzkim, za ich wiarę w mój rozsądek i podejmowane decyzje, oraz za nieposądzenie mnie o szaleństwo, gdy dowiedzieli się o zamiarze zrobienia doktoratu. To dzięki Wam będąc dzieckiem dowiedziałam się czym jest CERN, więc Wam również część tego doktoratu się należy.

Dziękuję mojej siostrze, Pauli Sak, za wskazówki dotyczące projektu okładki, oraz za inne, znacznie poważniejsze projekty architektoniczne i porady artystyczne udzielone mi w trakcie tego doktoratu.

Enfin et surtout, je voudrais remercier mon copain, David Porret, pour son approche scientifique envers mon humeur très changeante à la fin du doctorat et ainsi que sa tranquillité, sa gentillesse et son soutien, quand c'était le plus nécessaire. Merci Davidku!

I sincerely apologize if there is anyone I have forgotten to mention...

Thank you! 




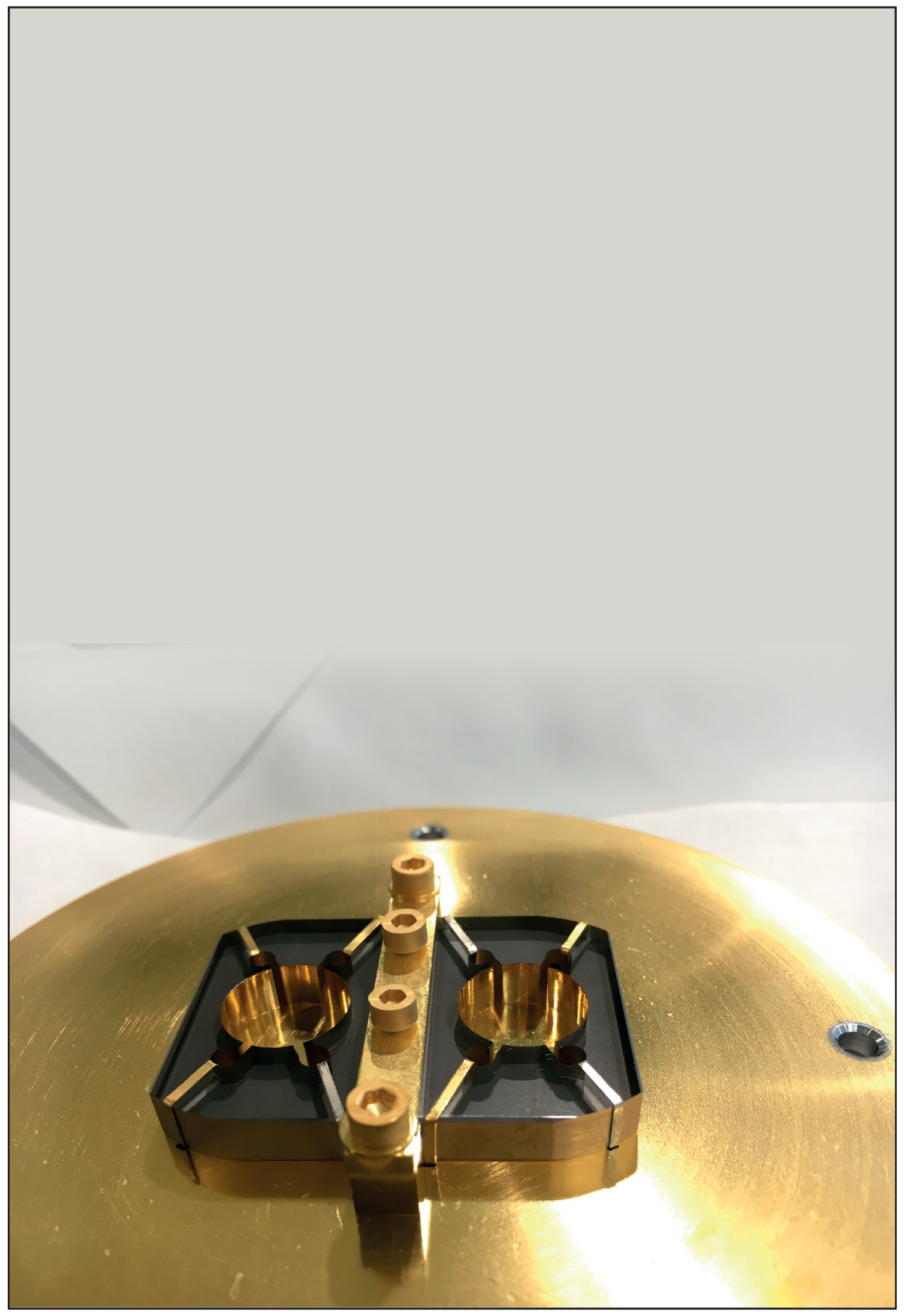

